\title{
Temporal aspects of cyclic messenger signaling in object recognition memory: a pharmalogical approach
}

Citation for published version (APA):

Akkerman, S. (2016). Temporal aspects of cyclic messenger signaling in object recognition memory: a pharmalogical approach. [Doctoral Thesis, Maastricht University]. Maastricht University. https://doi.org/10.26481/dis.20160324sa

Document status and date:

Published: 24/03/2016

DOI:

10.26481/dis.20160324sa

Document Version:

Publisher's PDF, also known as Version of record

\section{Please check the document version of this publication:}

- A submitted manuscript is the version of the article upon submission and before peer-review. There can be important differences between the submitted version and the official published version of record.

People interested in the research are advised to contact the author for the final version of the publication, or visit the DOI to the publisher's website.

- The final author version and the galley proof are versions of the publication after peer review.

- The final published version features the final layout of the paper including the volume, issue and page numbers.

Link to publication

\footnotetext{
General rights rights.

- You may freely distribute the URL identifying the publication in the public portal. please follow below link for the End User Agreement:

www.umlib.nl/taverne-license

Take down policy

If you believe that this document breaches copyright please contact us at:

repository@maastrichtuniversity.nl

providing details and we will investigate your claim.
}

Copyright and moral rights for the publications made accessible in the public portal are retained by the authors and/or other copyright owners and it is a condition of accessing publications that users recognise and abide by the legal requirements associated with these

- Users may download and print one copy of any publication from the public portal for the purpose of private study or research.

- You may not further distribute the material or use it for any profit-making activity or commercial gain

If the publication is distributed under the terms of Article $25 \mathrm{fa}$ of the Dutch Copyright Act, indicated by the "Taverne" license above, 


\section{TEMPORAL ASPECTS \\ OF CYCLIC MESSENGER SIGNALING IN OBJECT RECOGNITION MEMORY A PHARMACOLOGICAL APPROACH}


Copyright (C) Sven Akkerman, Utrecht, 2016

ISBN: 978-94-91602-50-4

Temporal aspects of cyclic messenger signaling in object recognition memory: a pharmacological approach

All rights reserved. No part of this thesis may be reproduced, stored in a retrieval database or published in any form or by any means, electronic, mechanical or photocopying, recording or otherwise, without the prior written permission of the author or, when appropriate, from the copyright-owning journals of previously published chapters.

Cover design by Sven Akkerman

Typesetting and layout by Sven Akkerman

Printed by Printservice Ede 


\title{
TEMPORAL ASPECTS \\ OF CYCLIC MESSENGER SIGNALING IN OBJECT RECOGNITION MEMORY A PHARMACOLOGICAL APPROACH
}

\author{
PROEFSCHRIFT
}

Ter verkrijging van de graad van doctor aan de Universiteit Maastricht op gezag van de Rector Magnificus Prof. Dr. L.L.G. Soete volgens het besluit van het College van Decanen in het openbaar te verdedigen op donderdag 24 maart 2016 om 10.00 uur

door

\section{SVEN AKKERMAN}

Geboren op 15 mei 1983 te Utrecht 


\section{PROMOTOR}

Prof. Dr. H.W.M. Steinbusch

\section{COPROMOTORES}

Dr. J. Prickaerts

Dr. A. Blokland

\section{BEOORDELINGSCOMMISSIE}

Prof. Dr. E.A.J. Joosten (voorzitter)

Dr. R. Havekes (Rijksuniversiteit Groningen)

Prof. Dr. R. D'Hooge (Katholieke Universiteit Leuven)

Dr. D.L.A. van den Hove

Prof. Dr. J.G. Ramaekers 
VOOR MIJN LIEVE RIANNE EN ONZE KLEINE SALAMANDER 



\section{CONTENT}

\section{Chapter 1}

GENERAL INTRODUCTION

Chapter 2

ObJeCt RECOGNITION TESTING: Methodological CONSIDERATIONS

ON EXPLORATION AND DISCRIMINATION MEASURES

\section{Chapter 3}

OBJECT RECOGNITION TESTING: STATISTICAL CONSIDERATIONS

\section{Chapter 4}

PDE5 INHIBITION IMPROVES ACQUISITION PROCESSES AFTER LEARNING

VIA A CENTRAL MECHANISM

\section{CHAPTER 5}

POSSIBLE OVERLAPPING TIME FRAMES OF ACQUISITION AND CONSOLIDATION

PHASES IN OBJECT MEMORY PROCESSES: A PHARMACOLOGICAL APPROACH

\section{Chapter 6}

MIND THE GAP: DELAYED MANIFESTATION OF LONG-TERM OBJECT MEMORY

IMPROVEMENT BY PHOSPHODIESTERASE INHIBITORS

\section{Chapter 7}

PDE5 INHIBITION IMPROVES OBJECT MEMORY IN STANDARD HOUSED RATS BUT

NOT IN RATS HOUSED IN AN ENRICHED ENVIRONMENT: IMPLICATIONS FOR

MEMORY MODELS?

\section{Chapter 8}

General Discussion

\section{SUMMARY}

\section{APPENDICES}

VALORISATION ADDENDUM

ABOUT THE AUTHOR

LIST OF PUBLICATIONS

DANKWOORD 



\section{ABBREVIATIONS}

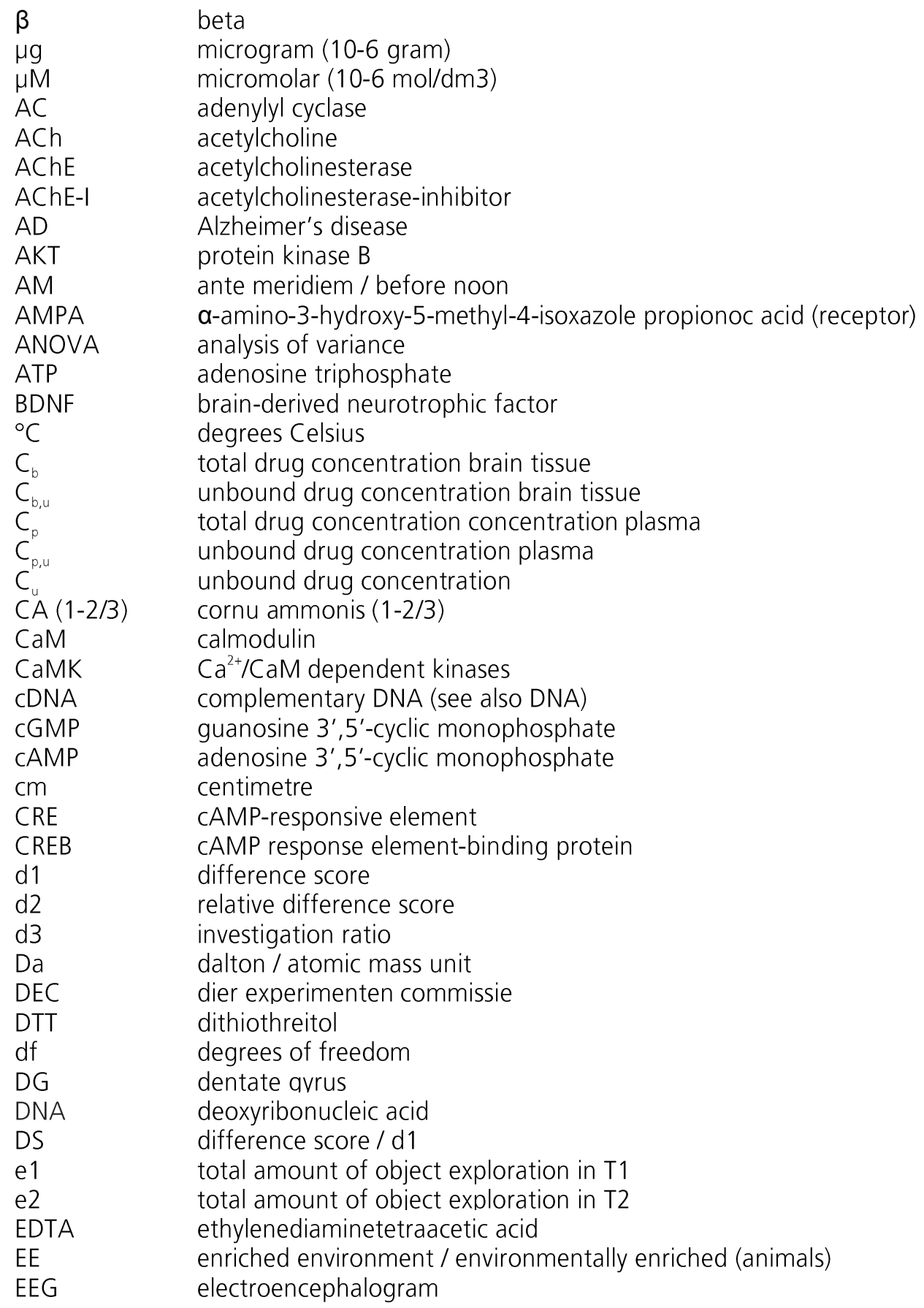




$\begin{array}{ll}\text { E-LTP } & \text { early-LTP (see also LTP) } \\ \text { EPM } & \text { elevated plus maze } \\ \text { ERK } & \text { extracellular-signal-regulated kinase(s) } \\ \text { F } & \text { F statistic } \\ \text { F }_{\text {G }} & \text { unbound fraction (of a drug) } \\ \text { G } & \text { gravitational constant } \\ \text { GABA } & \text { Y-aminobutyric acid } \\ \text { GAPDH } & \text { glyceraldehyde 3-phosphate dehydrogenase } \\ \text { GluR } & \text { glutamate receptor } \\ \text { h } & \text { hour(s) } \\ \text { Hprt } & \text { hypoxanthine-quanine phosphoribosvltransferase } \\ \text { HSD } & \text { Tukey's honest significant difference test } \\ \text { IC50 } & \text { the half max inhibitory concentration } \\ \text { ICV } & \text { intracerebroventricular / ventricular administration } \\ \text { i.e. } & \text { id est / that is } \\ \text { IP } & \text { intra peritoneal / peritoneal administration } \\ \text { IR } & \text { investigation ratio / d3 } \\ \text { kDA } & \text { kilodalton } \\ \text { kg } & \text { kilogram } \\ \text { LC-MS/MS } & \text { liquid chromatography tandem mass spectrometry } \\ \text { LLOQ } & \text { lower limit of quantification } \\ \text { L-LTP } & \text { late-LTP (see also LTP) } \\ \text { LTM } & \text { long term memory } \\ \text { LTP } & \text { long-term potentiation } \\ \text { lux } & \text { unit of illuminance / lumens per square metre } \\ \text { MAP } & \text { mitogen-activated protein kinase(s) } \\ \text { mBDNF } & \text { mature BDNF (see also BDNF) } \\ \text { mg } & \text { milligram (10 } \\ \text { min } & \text { minute(s) } \\ \text { ml } & \text { millilitre (10-3 litre) } \\ \text { mRNA } & \text { messenger RNA } \\ \text { M } & \text { molar } \\ \text { mRNA } & \text { messenger RNA (see also RNA) } \\ \text { MTL } & \text { medial temporal lobe } \\ \text { MW } & \text { molecular weight } \\ \text { MWM } & \text { Morris water maze } \\ \text { n } & \text { number of cases } \\ \text { NA } & \text { not applicable } \\ \text { NA } & \text { numerical aperture } \\ \text { ND } & \text { not determined } \\ \text { ng } & \text { nanogram (10-9 gram) } \\ \text { nM } & \text { nanomolar (10-9 mol/dm }{ }^{3} \text { ) } \\ \text { NMDA } & \text { N-methyl-D-aspartate } \\ \text { NMDAR } & \text { N-methyl-D-aspartate receptor } \\ \text { nmol } & \text { nanomol } \\ \text { NO } & \text { nitric oxide } \\ \text { NOP } & \text { novel object preference } \\ & \end{array}$




$\begin{array}{ll}\text { NOR } & \text { novel object recognition } \\ \text { NOS } & \text { nitric oxide synthase } \\ \text { n.S. } & \text { not significant } \\ \text { ORT } & \text { object recognition test / task } \\ \text { p } & \text { general symbol of probability } \\ \text { PBS } & \text { phosphate-buffered saline } \\ \text { PCREB } & \text { phosphorylated CREB (see also CREB) } \\ \text { PDE } & \text { phosphodiesterase } \\ \text { PDE-I } & \text { phosphodiesterase-inhibitor } \\ \text { pGSK-3B } & \text { phosphorylated glycogen synthase kinase 3 beta } \\ \text { pH } & \text { power of hydrogen / acidity } \\ \text { PK } & \text { pharmacokinetic } \\ \text { PKA } & \text { protein kinase A } \\ \text { PKG } & \text { protein kinase G } \\ \text { PM } & \text { post meridiem / after noon } \\ \text { PO } & \text { per os / oral administration } \\ \text { PRP } & \text { plasticitv related protein } \\ \text { PSD95 } & \text { postsynaptic density protein 95 } \\ \text { PTSD } & \text { post traumatic stress disorder } \\ \text { PVC } & \text { polyvinyl chloride } \\ \text { GPCR } & \text { quantitative polymerase chain reaction } \\ \text { RAM } & \text { radial arm maze } \\ \text { RDS } & \text { relative difference score / d2 } \\ \text { RNA } & \text { ribonucleic acid } \\ \text { RT-qPCR } & \text { real-time qPCR (see also qPCR) } \\ r & \text { correlation coefficient } \\ \text { S } & \text { second(s) } \\ \text { SDS-PAGE } & \text { sodium dodecyl sulfate - polyacrylamide gel electrophoresis } \\ \text { SEM } & \text { standard error of the mean } \\ \text { SGC } & \text { soluble guanylyl cyclase } \\ \text { SOL } & \text { solitary housed (animals) } \\ \text { SOC } & \text { socially housed (animals) } \\ \text { STFP } & \text { social transmission food preference (task) } \\ \text { STM } & \text { short term memory } \\ \text { SWR } & \text { sharp wave/ripple (EEG phenomenon) } \\ \text { SWS } & \text { slow wave sleep (EEG phenomenon) } \\ t & \text { Student's t statistic } \\ \text { T1 } & \text { first trial / learning trial } \\ \text { T2 } & \text { second trial / test trial } \\ \text { TBI } & \text { traumatic brain injury } \\ \text { TBS } & \text { tris-buffered saline } \\ \text { Tylose } & \text { methyl-cellulose } \\ \text { W } & \text { watt } \\ & \end{array}$




\section{Chapter 1}


GENERAL INTRODUCTION 


\section{MEMORY}

Memory is a cognitive function that is vital for normal daily life functioning. The ability to store and re-use information gives us the ability to learn from past experiences and shapes our identity. This makes memory crucial for individuals to live an independent life. Case reports of patients with brain lesions, like the famous H.M., have taught us that there are different forms of long-term memory that are more or less independent and are situated in distinct anatomical brain regions (Eichenbaum, 2013; Squire \& Zola, 1996). Long term memory can be subdivided into two major categories, declarative memory and non-declarative memory, which in turn contain multiple different sub-categories. All types of non-declarative memory are unconscious and manifest themselves via performance changes, for example motor learning. Declarative memory is the most important memory form for everyday interaction with our environment, as it is the only memory form that is consciously accessible. Patient studies have revealed that declarative memory depends on brain structures in the medial temporal lobe (MTL) including the hippocampal formation, entorhinal, perirhinal and parahippocampal cortices) and midline diencephalic brain structures like the hippocampus (Squire \& Zola, 1996). Declarative memory itself can be further subdivided into 2 types, semantic memory and episodic memory. The latter holds information about unique personal experiences and typically include information about time, place and about the event itself (Dickerson \& Eichenbaum, 2010). It is declarative memory that is typically the first type of memory to be affected in neurological disorders (Jahn, 2013).

\section{COGNITIVE IMPAIRMENT}

Forgetting or retention loss is a normal characteristic of memory as the majority of the information that we encounter is quickly forgotten. This is believed to be a useful characteristic, because it would be uneconomic to store numerous irrelevant details about experiences. As we get older we get more skilled at forgetting and making new memories becomes harder. Memory problems related to normal aging do not necessarily pose a large burden on an individual's quality of life. However, when 
declarative memory becomes severely impaired this is called amnesia. Amnesia can have many causes, like traumatic brain injuries (TBI) (Marshman, Jakabek, Hennessy, Quirk, \& Guazzo, 2013), malnutrition, sleep deprivation and severe stress. Although it is the hallmark symptom of Alzheimer's disease (AD) there is a host of neuropsychiatric disorders that can cause to amnesia. These include epilepsy, posttraumatic stress disorder (PTSD), depression, schizophrenia (Dickerson \& Eichenbaum, 2010), Korsakoff's syndrome (Kopelman, Thomson, Guerrini, \& Marshall, 2009), Parkinson's disease (Svenningsson, Westman, Ballard, \& Aarsland, 2012) and Huntington's disease (Walker, 2007). When amnesia results in a global loss of cognitive functioning we speak of dementia (Hirai, 2001). Dementia originates from the Latin words de- and ment-, which literally mean 'without mind'. The psychological impact of cognitive impairment goes far beyond the individual, as patients become heavily dependent on relatives and professional caregivers (Bynum, 2014). Hence, the economic burden on society is enormous, especially in western societies where people have a long life expectancy. It is estimated that 5.2 million Americans currently have $A D$ and this number is expected to grow to 13.8 million around 2050 . The total costs in 2014 for healthcare for people aged 65 years and older with dementia are calculated to be $\$ 214$ billion. In 2013 , more than 15 million family members and other unpaid caregivers were estimated to have provided 17.7 billion hours of care to people with $A D$ and other dementias, an additional indirect cost valued around $\$ 220$ billion in man hours (Association, 2014). These examples underline the magnitude of the social and economic burden of cognitive impairment on our society and the urgent need to develop effective treatments.

Several drugs are currently in used for the treatment of $A D$. The acetylcholinesterase (AChE) inhibitors donepezil, galantamine and rivastigmine, and the glutamatergic antagonist memantine (Stella, Radanovic, Canineu, de Paula, \& Forlenza, 2015). However, these present drug treatments for amnesia/dementia are largely symptomatic and have no significant impact on the long term-progression of the disease (O'Brien, Burns, \& Group, 2011). Therefore, there is a great need to further investigate the basal memory mechanisms that are involved, so that new and more effective treatments can be developed. When improving memory one should consider 
what aspect of memory should be targeted. In severe neurodegenerative diseases, like $A D$, declarative memory is usually the first type of memory to be affected (Jahn, 2013).

\section{BIOLOGICAL MECHANISMS UNDERLYING MEMORY}

The formation of declarative memory requires a sequence of complementary processes to register, store/maintain and retrieve events. Therefore, it is common to segregate memory processes into an acquisition, consolidation and retrieval phase. The first two phases are crucial for the storage of information about new experiences and successful retrieval is essential to utilize previously stored information. After retrieval, memories are again consolidated into the semantic structure, a process called re-consolidation. The consolidation of memories is dependent on different molecular cascades at different sites in the brain (Izquierdo et al., 2006). The neurotransmitter glutamate and its NMDA ( $N$-methyl-D-aspartate) and AMPA ( $\alpha$ amino-3-hydroxy-5-methyl-4-isoxazole propionoc acid) receptors have been shown to be crucially involved in different memory functions (Dere, Huston, \& De Souza Silva, 2007). Glutamatergic signaling regulates processes affecting the structure and strength of synaptic connections, or synaptic plasticity, which are believed to be the biological mechanism underlying learning and memory (Bailey, Kandel, \& Harris, 2015; Miyashita, Kubik, Lewandowski, \& Guzowski, 2008). One of these processes is called long-term potentiation (LTP), which essentially is an increase in synaptic transmission due to protein synthesis. Studies towards LTP have revealed that there are different phases within the LTP process, an early- and a late phase. Therefore, LTP is commonly divided in two phases; early-LTP (E-LTP) and late-LTP (L-LTP) (Reymann \& Frey, 2007).

\section{EARLY-LTP}

When an action potential arrives at a synapse, E-LTP is the first LTP stage that is induced. E-LTP is a result post translational modification of pre-existing proteins and independent of protein and RNA synthesis. E-LTP is maintained for minutes up to $<1-3$ 
hours after stimulation and is believed to underlie short term memory (Bekinschtein et al., 2007; Reymann \& Frey, 2007; Routtenberg \& Rekart, 2005).

For the induction of LTP glutamatergic co-activation of AMPA and NMDA receptors is necessary. Sufficient activation of postsynaptic AMPA receptors removes the $\mathrm{Mg}^{2+}$ blockage of the NMDA receptors resulting in an influx of $\mathrm{Ca}^{2+}$ (Reymann \& Frey, 2007). $\mathrm{Ca}^{2+}$, once inside the neuron, triggers multiple processes including the synthesis of nitric oxide (NO), stimulation of calmodulin (CaM). NO is primarily an intracellular messenger, however it is also capable of cross the cell membrane and reach target cells up to $0.4 \mathrm{~mm}$ away thereby functioning as a retrograde messenger. NO is synthesized by nitric oxide synthase (NOS) and has been implicated to modulate LTP and neurotransmitter release (Prast \& Philippu, 2001). Its effects on LTP are mainly exerted through soluble guanylyl cyclase ( $\mathrm{sGC}$ ) which in turn synthesizes guanosine $3^{\prime}, 5^{\prime}$-cyclic monophosphate (cGMP). Cyclic GMP activates protein kinase G (PKG) which phosphorylates CGMP-degrading PDE. After an initial increase, this negative feedback loop results in decreased cGMP levels below baseline for 60 minutes (Monfort, Munoz, Kosenko et al., 2004). Another effect of PKG is that it indirectly stimulates CREB phosphorylation, resulting in protein synthesis (Monfort, Munoz, \& Felipo, 2004). Acting as retrograde messenger, NO increases pre-synaptic glutamate release through sGC and cGMP (Jos Prickaerts et al., 2004). NO also enhances the release of several neurotransmitters, including glutamate, GABA and serotonin (Domek-Lopacinska \& Strosznajder, 2008; Prast \& Philippu, 2001). Ca²+CaM dependent kinases (CaMK) regulate adenylyl cyclases (AC), membrane bound enzymes which convert intracellular adenosine triphosphate (ATP) into cyclic adenosine monophosphate (CAMP) (Wang \& Storm, 2003). Moreover, CaMKs (especially CaMKII) modulate 'synaptic tagging'. Synaptic tagging involves the capture or processing of plasticity related proteins (PRPs) and is crucial for the transition from E-LTP into L-LTP. CaMKII also phosphorylates glutamate receptors (GluRs) (Reymann \& Frey, 2007). Both NMDA- and AMPA receptor are potentiated at least for the first 4 hours during LTP, the increase in AMPA response being twice the size of the increase of NMDA response (Dozmorov et al., 2006). After this initial E-LTP there is the L-LTP stage. 


\section{LATE-LTP}

L-LTP lasts more than 3 hours and requires stronger activation compared to E-LTP. Protein synthesis (including BDNF) and gene transcription produce neuronal changes that can last for months, making L-LTP a likely biological mechanism underlying long term memory formation (Bekinschtein et al., 2007; Raymond, 2007). Protein kinase A (PKA) is crucial for L-LTP. Decreased PKA activity significantly reduces L-LTP and impairs long-term memory, while leaving short-term memory intact (Abel et al., 1997). PKA is activated by NMDA-induced CAMP (Hajjhussein, Suvarna, Gremillion, Chandler, \& O'Donnell, 2007). Additional PKA activation through modulatory input, like activation of the dopaminergic $D_{1}$ and $D_{5}$ receptors, might be necessary for L-LTP formation (Raymond, 2007). These receptors activate G-proteins, which interact with AC. PKA phosphorylates CAMP-responsive element-binding protein (CREB) with phosphorylation levels peaking at 45 minutes and 6 hours after LTP induction (Ahmed \& Frey, 2005). Immediate-response genes are activated by phosphorylated CREB ( $p C R E B)$ through cAMP-responsive element (CRE) in their promoter regions, eventually leading to changes in synaptic structure (Cortes-Mendoza, Diaz de Leon-Guerrero, Pedraza-Alva, \& Perez-Martinez, 2013).

\section{PhOSPHOdiesterAses AND MEMORY}

Phosphodiesterases (PDEs) are modulatory enzymes that, via hydrolysis, regulate the intracellular levels of cGMP and CAMP, which are important proteins in memory formation. They are inactivated through hydrolytic cleavage of their 3'-phosphoester bonds, a process called hydrolysis (van Staveren, Markerink-van Ittersum, Steinbusch, \& de Vente, 2001). Presently, 11 families of PDEs have been discovered (PDE1 PDE11) and each family contains several different isoforms. PDEs are found everywhere in the body, with the exception of PDE6 and PDE11. These are the only PDE families that are not present in the brain (Blokland, Schreiber, \& Prickaerts, 2006; Reneerkens, Rutten, Steinbusch, Blokland, \& Prickaerts, 2009). The PDE families differ in localization and extend to which they hydrolyze CAMP and/or CGMP. Several PDE families have been implicated in memory processes; amongst these are the PDE2, 
PDE4 and PDE5 families (Rutten et al., 2007) which have characteristic properties that were utilized in this thesis. PDE5 selectively hydrolyzes CGMP, PDE4 is CAMP specific, and PDE2 hydrolyzes both CAMP and cGMP (Boess et al., 2004; Halene \& Siegel, 2007). Interestingly, PDEs are also influenced by CGMP, which has been found to stimulate CAMP hydrolyzing PDE activity (Oleshansky, 1980).

\section{PHOSPHODIESTERASE INHIBITORS}

There is a specific class of drugs that act via the inhibition of PDE's, the so called phosphodiesterase inhibitors (PDE-Is). There are selective PDE-Is that only inhibit one particular PDE family. For example, vardenafil only inhibits PDE5 (Reneerkens, Rutten, Steinbusch, Blokland, \& Prickaerts, 2008). However, there are also non-selective PDEIs, like caffeine, that inhibit multiple PDE families and also have other targets besides PDE's (Essayan, 1999). Therefore, by inhibiting a specific PDE isoform with PDE-Is one can increase the intracellular levels of CAMP and/or CGMP, which, in turn. This will, in turn, lead to increased levels of PKG and PKA, which leads to phosphorylation of CREB and stronger LTP. For this reason, PDE-Is might have potential for the treatment of amnesia and other psychological disorders affecting cognitive function (Blokland et al., 2006; Garcia-Osta, Cuadrado-Tejedor, Garcia-Barroso, Oyarzabal, \& Franco, 2012). Several studies have demonstrated that PDE-Is are capable of improving memory animal models, the effects being strongly dependent on the timing of administration in relation to time of learning (Blokland et al., 2006; J. Prickaerts, de Vente, Honig, Steinbusch, \& Blokland, 2002; Rutten et al., 2007). On the other hand, in human studies convincing evidence for cognition enhancing properties of PDE-Is is yet to be found (Grass et al., 2001; Reneerkens et al., 2013; Schultheiss et al., 2001).

Because of their unique properties PDE-Is can exert effects on different stages of memory formation, making them interesting tools for the investigation of these different stages. For example, object memory can be improved by administering a CGMP specific PDE5-I prior to learning or directly after learning trial, while PDE5-Is are unable to improve memory function when administered at a later stage of memory consolidation (Prickaerts et al, 2004; Rutten, Prickaerts, Hendrix, van der Staay, Şik, \& 
Blokland, 2007 ${ }^{b}$ ). The CAMP specific PDE4-Is, on the other hand, effectively improve object recognition when administered either directly after a learning trial or 3 hours later. When PDE2, which hydrolyzes both CGMP and CAMP, is inhibited, memory can be improved with administrations either directly after learning or $3 \mathrm{~h}$ after learning (Rutten et al., 2007). Current pharmacological treatments for cognitive impairments are mainly focusing on specific extracellular messengers, including the cholinergic and glutamatergic neurotransmission (Stella et al., 2015). PDE-I's have the advantage that they can directly target a broad spectrum of intracellular processes.

\section{OBJECT RECOGNITION TASK}

Memory can be tested in wide array of behavioral animal paradigms. One-trial learning paradigms, such as the passive avoidance test, fear conditioning and the object recognition test (ORT) enable researchers to evaluate noötropic drug effects on individual memory components. Typically, these tests consist of an acquisition trial in which information is presented, and a test trial in which the information has to be retrieved. Because the performance in the ORT is dependent on medial temporal lobe structures (Winters \& Bussey, 2005), it could be assumed that the task measures declarative memory or at least aspects of declarative memory (Ennaceur, 2010). As shown in Figure 1, the timing of drug administration in relation to the learning trial determines which memory process is affected (J. Prickaerts, Sik, van der Staay, de Vente, \& Blokland, 2005). That is, when a drug is injected shortly before the acquisition trial it is able to influence acquisition processes, whereas if the drug is injected after the acquisition trial it is assumed to affect only consolidation processes. When a drug is administered shortly before the test trial it will only have an effect on retrieval. The exact timing of drug administration is largely dependent of the activity profile of the target and the pharmacokinetics of the drug itself. This implicates that a drug, by using alternate timings of administration within a one-trail learning task, can be used as a tool to investigate the temporal activity profile of the targets of the drug in question. However, to properly investigate temporal processes in this manner, one needs to understand the pharmacokinetic profile of the drug, which can greatly differ between animal species. One of the properties that distinguish the ORT from other 
one-trial learning paradigms is the fact that it is based on the natural tendency of animals to explore novelty in their environment. This means that animals don't need any pre-test training of negative reinforcement to perform the test. Many different variants of the ORT are used and there is little consensus about the correct way to analyze the readout parameters or even what exact type of memory is measured in the ORT. These are issues that need to be addressed as the ORT is a widely used tool for pre-clinical memory assessment and has unique qualities that allow for the specific timing of interventions under low-stress conditions.

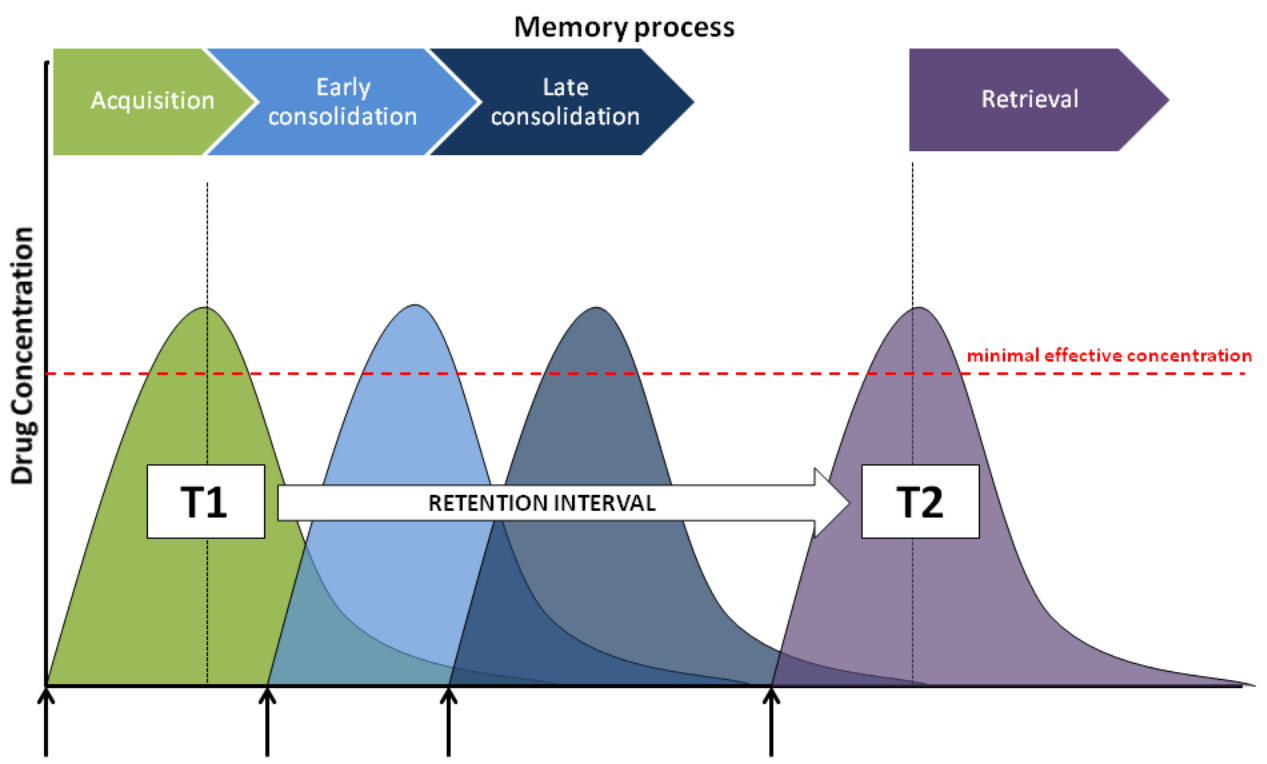

Time of Administration

Figure 1 The importance of drug administration timing in the ORT

The ORT can be used to manipulate memory processes separately. When a drug is injected shortly before the acquisition trial (T1) it is able to influence acquisition processes, whereas if the drug is injected after the acquisition trial it is assumed to affect only consolidation processes. When a drug is administered shortly before the test trial (T2) it will only have an effect on retrieval. It is crucial to determine the pharmacokinetics as they will determine the duration of the effective window as well as the onset of the effects of the drug, in combination with the minimum concentration required for the drug to become biologically active. This concentration will vary between compounds. 


\section{AIM AND OUTLINE OF THE THESIS}

Taken together it has been shown that PDE-Is can improve memory by raising CAMP and cGMP levels and exert very robust effects on memory in animal models. Yet, in human studies PDE-I inhibitors have not yet proven their potential as cognition enhancers. There may be several reasons why clinical studies towards the cognition enhancing potential of PDE-I's have not been able to replicate the promising results of the pre-clinical animal studies. It may be the case that we are not correctly interpreting the readout parameters of cognitive animal tests and therefore draw unjustified conclusions about what they mean. It may also be the case that our animal models lack predictive validity for the assessment of drug effects on memory. Furthermore, translational difficulties of pre-clinical PDE-I results to humans may be due to lack of understanding of the underlying biological processes, pharmacokinetics and the temporal outline of memory expression. The goal of this thesis is to address these questions and further our understanding of the processes underlying the effects PDE-Is on memory.

In this thesis we extensively used the ORT, which is a widely used method to assess memory performance in animals. Because it is an intuitive one-trail learning paradigm, the task allows for the precise investigation of different memory processes without any negative reinforcement which could influence normal memory formation. However, there are many different variants of the ORT and there is debate about the exact memory type that is measured and the relationship between exploration and discrimination measures. Furthermore, it is not clear understanding about the necessity of pre-experimental habituation and the effects of habituation on ORT performance. To get a clear understanding about the ORT variant used in our lab, we performed a meta-analysis, including naïve and vehicle conditions of over 50 studies with Wistar rats that were previously performed in our lab (Chapter 2).

Several different discrimination indices can be calculated from the object exploration levels, which are the readout parameters of the ORT. In the literature there is a lack of consensus about which discrimination index is most suitable for the assessment of memory performance and it is unclear how these discrimination parameters compare 
to one another. In this thesis we compared the different discrimination indices, to gain further insight in how to properly analyze and interpret results of the ORT (Chapter 3).

Previous studies have shown, PDE4-Is and PDE5-Is have to be injected at very specific time points in relation to the time of learning. These specific administration times only provide a rough indication about the active window of their specific intracellular targets. In this thesis we wanted to further elaborate the signaling windows of CAMP and CGMP in vivo. Therefore, we investigated the pharmacokinetic profiles of the PDE4-I rolipram and PDE5-I vardenafil and tested these drugs in the ORT to find the earliest and latest effective time of injection, hence revealing the complete window of action of CAMP and CGMP (Chapter $\mathbf{4}$ and 5).

Memories are not constant over time, as the quality of most memorized events seems to degrade over time. It is also a widely accepted notion that long term memories (LTM) have to be physically incorporated in the synaptic architecture of the brain. These structural changes in the brain need to be incorporated over time, a process that lasts for hours or even days. There is not much research about the actual expression of memories during these initial stages of LTM. Possible fluctuation in memory expression may cause confusion about cognition enhancing effects of drugs, because possible effects can be missed or the extent of an effect can be misjudged. In this chapter we investigated natural forgetting in the ORT in the first $24 \mathrm{~h}$ hours after learning and consequently, within these initial $24 \mathrm{~h}$, studied the expression profiles of object memory enhanced by vardenafil and rolipram, which are specific PDE5 and PDE4 inhibitors, respectively (Chapter 6).

Many pre-clinical studies that have shown the potential of PDE-Is a cognition enhancing drugs. However, there convincing evidence about their effectiveness in humans has yet to be found. This is not only the case for PDE-I's but for cognition enhancers in general (Kola \& Landis, 2004). One of the causes of the poor predictive validity of the current animal models may be the highly impoverished and standardized living conditions of lab animals. It has been proposed that Environmental Enrichment may allow lab animals to have a healthier, more natural brain 
development, which may more closely resemble the human situation outside the lab. To explore whether environmental factors can explain the discrepancy between human- and animal studies and possibly develop a more valid (i.e. predictive) animal model of cognition, we investigated the effects of EE on the efficacy of vardenafil, which has proven itself as a cognition enhancer in rat housed under standardized laboratory conditions (Chapter 7).

Finally, our experimental findings are summarized and discussed in Chapter 8 . 


\section{REFERENCES}

Abel, T., Nguyen, P.V., Barad, M., Deuel, T.A., Kandel, E.R., \& Bourtchouladze, R. (1997). Genetic demonstration of a role for PKA in the late phase of LTP and in hippocampus-based long-term memory. Cell, 88(5), 615-626.

Ahmed, T., \& Frey, J.U. (2005). Plasticity-specific phosphorylation of CaMKII, MAPkinases and CREB during late-LTP in rat hippocampal slices in vitro. Neuropharmacology, 49(4), 477-492.

Association, A.s. (2014). 2014 Alzheimer's disease facts and figures. Alzheimers Dement, 10(2), 47-92.

Bailey, C.H., Kandel, E.R., \& Harris, K.M. (2015). Structural Components of Synaptic Plasticity and Memory Consolidation. Cold Spring Harb Perspect Biol, 7(7), a021758.

Bekinschtein, P., Cammarota, M., Igaz, L.M., Bevilaqua, L.R., Izquierdo, I., \& Medina, J.H. (2007). Persistence of long-term memory storage requires a late protein synthesis- and BDNF- dependent phase in the hippocampus. Neuron, 53(2), 261-277.

Blokland, A., Schreiber, R., \& Prickaerts, J. (2006). Improving memory: a role for phosphodiesterases. Curr Pharm Des, 12(20), 2511-2523.

Boess, F.G., Hendrix, M., van der Staay, F.-J., Erb, C., Schreiber, R., van Staveren, W. (2004). Inhibition of phosphodiesterase 2 increases neuronal cGMP, synaptic plasticity and memory performance. Neuropharmacology, 47(7), 1081-1092.

Bynum, J.P. (2014). The long reach of Alzheimer's disease: patients, practice, and policy. Health Aff (Millwood), 33(4), 534-540.

Cortes-Mendoza, J., Diaz de Leon-Guerrero, S., Pedraza-Alva, G., \& Perez-Martinez, L. (2013). Shaping synaptic plasticity: the role of activity-mediated epigenetic regulation on gene transcription. Int J Dev Neurosci, 31(6), 359-369.

Dere, E., Huston, J.P., \& De Souza Silva, M.A. (2007). The pharmacology, neuroanatomy and neurogenetics of one-trial object recognition in rodents. Neuroscience \& Biobehavioral Reviews, 31(5), 673-704.

Dickerson, B.C., \& Eichenbaum, H. (2010). The episodic memory system: neurocircuitry and disorders. Neuropsychopharmacology, 35(1), 86-104.

Domek-Lopacinska, K., \& Strosznajder, J.B. (2008). The effect of selective inhibition of cyclic GMP hydrolyzing phosphodiesterases 2 and 5 on learning and memory processes and nitric oxide synthase activity in brain during aging. Brain Res, $1216,68-77$.

Dozmorov, M., Li, R., Abbas, A.K., Hellberg, F., Farre, C., Huang, F.S. (2006). Contribution of AMPA and NMDA receptors to early and late phases of LTP in hippocampal slices. Neurosci Res, 55(2), 182-188. 
Eichenbaum, H. (2013). What H.M. taught us. J Cogn Neurosci, 25(1), 14-21.

Ennaceur, A. (2010). One-trial object recognition in rats and mice: Methodological and theoretical issues. Behavioural Brain Research, 215(2), 244-254.

Essayan, D.M. (1999). Cyclic nucleotide phosphodiesterase (PDE) inhibitors and immunomodulation. Biochemical Pharmacology, 57(9), 965-973.

Garcia-Osta, A., Cuadrado-Tejedor, M., Garcia-Barroso, C., Oyarzabal, J., \& Franco, R. (2012). Phosphodiesterases as therapeutic targets for Alzheimer's disease. ACS Chem Neurosci, 3(11), 832-844.

Grass, H., Klotz, T., Fathian-Sabet, B., Berghaus, G., Engelmann, U., \& Kaferstein, H. (2001). Sildenafil (Viagra): is there an influence on psychological performance? Int Urol Nephrol, 32(3), 409-412.

Hajjhussein, H., Suvarna, N.U., Gremillion, C., Chandler, L.J., \& O'Donnell, J.M. (2007). Changes in NMDA receptor-induced cyclic nucleotide synthesis regulate the age-dependent increase in PDE4A expression in primary cortical cultures. Brain Res, 1149, 58-68.

Halene, T.B., \& Siegel, S.J. (2007). PDE inhibitors in psychiatry; future options for dementia, depression and schizophrenia? Drug Discovery Today, 12, 870878.

Hirai, S. (2001). Distinction between Dementia and Memory Decline. JMAJ, 44(6), 5.

Izquierdo, I.n., Bevilaqua, L.R.M., Rossato, J.I., Bonini, J.S., Medina, J.H., \& Cammarota, M. (2006). Different molecular cascades in different sites of the brain control memory consolidation. Trends in Neurosciences, 29(9), 496-505.

Jahn, H. (2013). Memory loss in Alzheimer's disease. Dialogues Clin Neurosci, 15(4), 445-454.

Kola, I., \& Landis, J. (2004). Can the pharmaceutical industry reduce attrition rates? Nat Rev Drug Discov, 3(8), 711-715.

Kopelman, M.D., Thomson, A.D., Guerrini, I., \& Marshall, E.J. (2009). The Korsakoff syndrome: clinical aspects, psychology and treatment. Alcohol Alcohol, 44(2), 148-154.

Marshman, L.A., Jakabek, D., Hennessy, M., Quirk, F., \& Guazzo, E.P. (2013). Posttraumatic amnesia. J Clin Neurosci, 20(11), 1475-1481.

Miyashita, T., Kubik, S., Lewandowski, G., \& Guzowski, J.F. (2008). Networks of neurons, networks of genes: an integrated view of memory consolidation. Neurobiol Learn Mem, 89(3), 269-284.

Monfort, P., Munoz, M.D., \& Felipo, V. (2004). Hyperammonemia impairs long-term potentiation in hippocampus by altering the modulation of cGMP-degrading phosphodiesterase by protein kinase G. Neurobiol Dis, 15(1), 1-10.

Monfort, P., Munoz, M.D., Kosenko, E., Llansola, M., Sanchez-Perez, A., Cauli, O. (2004). Sequential activation of soluble guanylate cyclase, protein kinase $G$ 
and CGMP-degrading phosphodiesterase is necessary for proper induction of long-term potentiation in CA1 of hippocampus. Alterations in hyperammonemia. Neurochem Int, 45(6), 895-901.

O'Brien, J.T., Burns, A., \& Group, B.A.P.D.C. (2011). Clinical practice with antidementia drugs: a revised (second) consensus statement from the British Association for Psychopharmacology. J Psychopharmacol, 25(8), 997-1019.

Oleshansky, M.A. (1980). Inhibition by purine compounds of cyclic GMP-stimulated cyclic AMP phosphodiesterase activity from a particulate fraction of rat striatum. Life Sci, 27(12), 1089-1095.

Prast, H., \& Philippu, A. (2001). Nitric oxide as modulator of neuronal function. Prog Neurobiol, 64(1), 51-68.

Prickaerts, J., de Vente, J., Honig, W., Steinbusch, H.W., \& Blokland, A. (2002). cGMP, but not CAMP, in rat hippocampus is involved in early stages of object memory consolidation. Eur J Pharmacol, 436(1-2), 83-87.

Prickaerts, J., Sik, A., van der Staay, F.J., de Vente, J., \& Blokland, A. (2005). Dissociable effects of acetylcholinesterase inhibitors and phosphodiesterase type 5 inhibitors on object recognition memory: acquisition versus consolidation. Psychopharmacology (Berl), 177(4), 381-390.

Prickaerts, J., Sik, A., van Staveren, W.C.G., Koopmans, G., Steinbusch, H.W.M., van der Staay, F.J. (2004). Phosphodiesterase type 5 inhibition improves early memory consolidation of object information. Neurochemistry International, 45(6), 915-928.

Raymond, C.R. (2007). LTP forms 1, 2 and 3: different mechanisms for the "long" in long-term potentiation. Trends Neurosci, 30(4), 167-175.

Reneerkens, O.A., Rutten, K., Steinbusch, H.W., Blokland, A., \& Prickaerts, J. (2008). Selective phosphodiesterase inhibitors: a promising target for cognition enhancement. Psychopharmacology (Berl), 202(1-3), 419-443.

Reneerkens, O.A., Rutten, K., Steinbusch, H.W., Blokland, A., \& Prickaerts, J. (2009). Selective phosphodiesterase inhibitors: a promising target for cognition enhancement. Psychopharmacology (Berl), 202(1-3), 419-443.

Reneerkens, O.A., Sambeth, A., Ramaekers, J.G., Steinbusch, H.W., Blokland, A., \& Prickaerts, J. (2013). The effects of the phosphodiesterase type 5 inhibitor vardenafil on cognitive performance in healthy adults: a behavioralelectroencephalography study. J Psychopharmacol, 27(7), 600-608.

Reymann, K.G., \& Frey, J.U. (2007). The late maintenance of hippocampal LTP: requirements, phases, 'synaptic tagging', 'late-associativity' and implications. Neuropharmacology, 52(1), 24-40.

Routtenberg, A., \& Rekart, J.L. (2005). Post-translational protein modification as the substrate for long-lasting memory. Trends Neurosci, 28(1), 12-19. 
Rutten, K., Prickaerts, J., Hendrix, M., van der Staay, F.J., Sik, A., \& Blokland, A. (2007). Time-dependent involvement of CAMP and cGMP in consolidation of object memory: studies using selective phosphodiesterase type 2, 4 and 5 inhibitors. Eur J Pharmacol, 558(1-3), 107-112.

Schultheiss, D., Muller, S.V., Nager, W., Stief, C.G., Schlote, N., Jonas, U. (2001). Central effects of sildenafil (Viagra) on auditory selective attention and verbal recognition memory in humans: a study with event-related brain potentials. World J Urol, 19(1), 46-50.

Squire, L.R., \& Zola, S.M. (1996). Structure and function of declarative and nondeclarative memory systems. Proc Natl Acad Sci U S A, 93, 13515-13522.

Stella, F., Radanovic, M., Canineu, P.R., de Paula, V.J., \& Forlenza, O.V. (2015). Antidementia medications: current prescriptions in clinical practice and new agents in progress. Ther Adv Drug Saf, 6(4), 151-165.

Svenningsson, P., Westman, E., Ballard, C., \& Aarsland, D. (2012). Cognitive impairment in patients with Parkinson's disease: diagnosis, biomarkers, and treatment. Lancet Neurol, 11(8), 697-707.

van Staveren, W.C., Markerink-van Ittersum, M., Steinbusch, H.W., \& de Vente, J. (2001). The effects of phosphodiesterase inhibition on cyclic GMP and cyclic AMP accumulation in the hippocampus of the rat. Brain Res, 888(2), 275286.

Walker, F.O. (2007). Huntington's disease. Lancet, 369(9557), 218-228.

Wang, H., \& Storm, D.R. (2003). Calmodulin-regulated adenylyl cyclases: cross-talk and plasticity in the central nervous system. Mol Pharmacol, 63(3), 463-468.

Winters, B.D., \& Bussey, T.J. (2005). Transient Inactivation of Perirhinal Cortex Disrupts Encoding, Retrieval, and Consolidation of Object Recognition Memory. The Journal of Neuroscience, 25(1), 52-61. 



\section{ChAPTER 2}




\section{OBJECT RECOGNITION TESTING: METHODOLOGICAL CONSIDERATIONS ON EXPLORATION AND DISCRIMINATION MEASURES}

S. Akkerman, A. Blokland, O. Reneerkens, N.P. van Goethem, E. Bollen, H.J.M. Gijselaers, C.K.J Lieben, H.W.M. Steinbusch and J. Prickaerts' 


\section{ABSTRACT}

The object recognition task (ORT) is a popular one-trial learning test for animals. In the current study, we investigated several methodological issues concerning the task. Data was pooled from 28 ORT studies, containing 731 male Wistar rats. We investigated the relationship between 3 common absolute and relative discrimination measures, as well as their relation to exploratory activity. In this context, the effects of preexperimental habituation, object familiarity, trial duration, retention interval and the amnesic drugs MK-801 and scopolamine were investigated. Our analyses showed that the ORT is very sensitive, capable of detecting subtle differences in memory (discrimination) and exploratory performance. As a consequence, it is susceptible to potential biases due to (injection) stress and side effects of drugs. Our data indicated that a minimum amount of exploration is required in the sample and test trial for stable significant discrimination performance. However, there was no relationship between the level of exploration in the sample trial and discrimination performance. In addition, the level of exploration in the test trial was positively related to the absolute discrimination measure, whereas this was not the case for relative discrimination measures, which correct for exploratory differences, making them more resistant to exploration biases. Animals appeared to remember object information over multiple test sessions. Therefore, when animals have encountered both objects in prior test sessions, the object preference observed in the test trial of $1 \mathrm{~h}$ retention intervals is probably due to a relative difference in familiarity between the objects in the test trial, rather than true novelty per se. Taken together, our findings suggest to take into consideration pre-experimental exposure (familiarization) to objects, habituation to treatment procedures, and the use of relative discrimination measures when using the ORT. 


\section{INTRODUCTION}

Twenty-three years ago, Ennaceur and Delacour (Ennaceur \& Delacour, 1988) introduced a new one-trial learning test for neurobiological studies. Since its introduction it has been referred to as novel object preference (NOP) test (Gaskin, et al., 2010), novel object recognition (NOR) task (Schenk, Harper, \& Do, 2010) and object recognition task (ORT) (Ennaceur, Michalikova, Bradford, \& Ahmed, 2005). In the present paper we use the ORT abbreviation to refer to object recognition testing. The ORT is based on the underlying principle that, in a familiar environment, laboratory rodents show an instinctive attraction towards novelty, or neotic preference (Hughes, 2007). In general, animals are allowed to freely explore two identical sample objects during a sample- or learning trial. After a certain delay, the animals perform a test trial, in which they are confronted with one of the sample objects and a novel one. If rodents show more interaction with the novel object during the test trial, it can be presumed that they have remembered the sample object. This novel object preference is used as an indication of memory.

Because it utilizes the natural behavior of the animals, the ORT is relatively free of stress if properly administered. Furthermore the property of one-trial learning makes it suitable for the examination of temporal aspects of memory. Over time, it has become a widely used tool for the assessment of memory functions in combination with pharmacological treatments (Klinkenberg \& Blokland, 2010; Prickaerts, van Staveren, et al., 2002; Rutten, Prickaerts, Schaenzle, Rosenbrock, \& Blokland, 2008; Rutten, et al., 2009; Sambeth, Riedel, Smits, \& Blokland, 2007) and brain lesions (Ainge, et al., 2006; Albasser, Davies, Futter, \& Aggleton, 2009; Mumby, 2001; Mumby, Gaskin, Glenn, Schramek, \& Lehmann, 2002). The ORT is mainly used for rodent experiments, most research is done with rats and mice but the task is also suitable for testing other rodent species (van Goethem, et al., 2012). It has also been successfully applied using larger animal species like dogs (Callahan, Ikeda-douglas, Head, Cotman, \& Milgram, 2000), pigs (Gifford, Cloutier, \& Newberry, 2007; Kornum, Thygesen, Nielsen, Knudsen, \& Lind, 2007) and horses (Hanggi, 2010), making it a versatile tool suitable for inter-species comparisons. The most essential brain structures implicated in object 
recognition memory of rodents, are the hippocampus and rhinal cortices, the perirhinal and in particular the postrhinal cortex (Dickerson \& Eichenbaum, 2010). Of note, the human and primate counterpart of the postrhinal cortex is the parahippocampal cortex. Although there is much evidence that the hippocampus is implicated in object recognition testing (de Lima, Luft, Roesler, \& Schröder, 2006; Dere, Huston, \& De Souza Silva, 2007; O'Brien, Lehmann, Lecluse, \& Mumby, 2006), its role in processing of the actual object information is more an integrative one, i.e. combining object and contextual information into integral episodic memories (Dickerson \& Eichenbaum, 2010; B. D. Winters, Saksida, \& Bussey, 2008). Concerning object information itself, the perirhinal cortex is considered to be the most crucial structure whereas the postrhinal cortex is implicated in contextual information processing (Mumby, Piterkin, Lecluse, \& Lehmann, 2007; Winters, et al., 2008).

Many variations on the original task have been developed over time, making it difficult to compare results between different laboratories. Differences exist in object and arena features like the size, shape and materials. Also, differences in animal strain, age, gender, and housing conditions have been reported to influence object recognition (Andrews, 1996; Andrews, Jansen, Linders, Princen, \& Broekkamp, 1995; Frick \& Gresack, 2003; Sik, van Nieuwehuyzen, Prickaerts, \& Blokland, 2003; van Goethem, et al., 2012). Furthermore, different test protocols are being used. For example, some studies use flexible sample trial durations, allowing all animals to reach a specific amount of object interaction (Ainge, et al., 2006; Norman \& Eacott, 2004; Winters \& Bussey, 2005; Winters, Forwood, Cowell, Saksida, \& Bussey, 2004). Other experiments have fixed sample trial durations and consequently, each individual animal differs in the amount of sample object exposure (Albasser, et al., 2009; Ennaceur \& Delacour, 1988; Mumby, et al., 2007; Prickaerts, de Vente, Honig, Steinbusch, \& Blokland, 2002). Before testing starts, the animals are often familiarized or habituated and protocols differ greatly between laboratories. In some laboratories the animals are allowed to familiarize with only the apparatus (Ainge, et al., 2006; Albasser, et al., 2009; Gaskin, et al., 2010; O'Brien, et al., 2006), others also introduce objects in the pre-experimental phase (Mumby, et al., 2007) or even let the animals undergo the full testing procedure, including injections (Prickaerts, Sik, van der Staay, 
de Vente, \& Blokland, 2005; Rutten, et al., 2007). Even more fundamental, there is no consensus about the definition of object investigation. In the original test, object investigation was defined as directing the nose towards the object at a distance of 2 $\mathrm{cm}$ or less (Ennaceur \& Delacour, 1988). However, minimal proximities of $1 \mathrm{~cm}$ (Albasser, et al., 2009) and $4 \mathrm{~cm}$ (Gaskin, et al., 2010) have also been reported. Generally, climbing and leaning on objects is not considered to be object examination (Bevins \& Besheer, 2006), although some do in fact consider this to be relevant objectdirected behavior (Besheer \& Bevins, 2000; Gaskin, et al., 2010; Wilkinson, Herrman, Palmatier, \& Bevins, 2006).

It is also important to note that novel object preference is being analyzed and reported in various ways. Some authors calculate the absolute difference in exploration times between the novel and the sample object, resulting a difference score (DS). In others, object discrimination can also be reported in terms of the relative interaction with each object in the test trial, resulting in a percentage or investigation ratio (IR). Lastly, results of the ORT have also been reported as the relative difference score (RDS), which divides the DS by the total time spent exploring both the objects in the test trial. Theoretically, random exploration would result in a (R)DS of 0 or an IR of 0.5, a higher score indicates that an animal directed the majority of exploration towards the novel object, thereby implicating that it has remembered the sample object. Since studies generally report only one of the different discrimination measures it is difficult to directly compare them. To our knowledge, it has not yet been fully investigated, in one and the same study, how the different discrimination indices are related.

The aim of our study is to shed more light on the effects of familiarization and habituation to the procedures on the measures of exploration and discrimination in the ORT. Additionally, we want to deepen our understanding of the relationships between the different discrimination and exploration measures in the sample- and test trial. This could be helpful in determining the minimum amount of exploration required for reliable object discrimination, by this we mean the amount of exploration that represents normal exploratory behavior and allows for a meaningful statistical 
interpretation. We have included historical data from 28 ORT studies performed in our lab to test the potential of several drugs to reverse natural or drug-induced forgetting. To investigate the latter, $1 \mathrm{~h}$ retention intervals were used in combination with amnesic drugs, the non-competitive $N$-methyl-d-aspartate (NMDA) receptor antagonist MK-801 or the muscarinic receptor antagonist scopolamine. Both drugs have proven reliably to attenuate the novel object preference in a $1 \mathrm{~h}$ retention delay, likely due to drug induced memory impairment (de Lima, Laranja, Bromberg, Roesler, \& Schröder, 2005; Sambeth, et al., 2007; van der Staay, Rutten, Erb, \& Blokland, 2011). $24 \mathrm{~h}$ retention intervals were used to investigate drug effects on natural forgetting, as our male Wistar rats normally do not discriminate anymore between the novel and the familiar object after such an interval (Ennaceur \& Delacour, 1988; Prickaerts, de Vente, et al., 2002; Prickaerts, et al., 2005; Rutten, et al., 2007; Rutten, Prickaerts, et al., 2008).

\section{Methods}

\section{ANIMALS}

All experiments were performed using male Wistar rats ordered from Harlan (the Netherlands). The animals ( $n=731)$ were ordered at an age of 2 months and tested between 3 and 5 months of age. After arrival, animals were individually housed in standard MakrolonTM Type III cages with sawdust bedding. A cardboard tunnel with a diameter of $10 \mathrm{~cm}$, length of $12.5 \mathrm{~cm}$ and a $0.5 \mathrm{~cm}$ thick wall was provided as enrichment together with a piece of wood. Food and water were provided ad libitum, water was acidified ( $\mathrm{pH} 3$ ) to prevent bacterial infections and refreshed every week. Testing was done in the same room as where the animals were housed. During the first two weeks the rats were allowed to get accustomed to a reversed $12 \mathrm{~h}$ day/night cycle (lights were on from 19:00 till 07:00). After this they were habituated to handling and familiarized with the testing procedures. All testing was done during their active phase, i.e. during dark phase at daytime. During the dark phase red fluorescent tubes provided illumination for which the rat-retina is relatively insensitive. A radio played softly, providing background noise $24 \mathrm{~h}$ a day, also during testing. The 
room temperature was kept constant at $20^{\circ} \mathrm{C}$ and the humidity of the air was kept within a range of $60-80 \%$.

\section{APPARATUS}

The test setup that was used is a slightly adapted version of the object recognition task as described by Ennaceur and Delacour (1988). Instead of a square arena of 65 $\mathrm{cm} \times 45 \mathrm{~cm} \times 45 \mathrm{~cm}$, as used by Ennaceur and Delacour, a circular arena, with a diameter of $83 \mathrm{~cm}$ was used in our studies. The floor plate and back-half of the arena wall were made of gray (RAL 7035) polyvinyl chloride. The frontal half of the $40 \mathrm{~cm}$ high arena wall was made of transparent polyvinyl chloride to enable direct observation of the animals. Light intensity on the apparatus floor was about 20 lux. Illumination was provided by red fluorescent tubes in the ceiling and a $40 \mathrm{~W}$ light bulb, which was switched on during behavioral observations, in the corner of the room covered by a lamp-shade.

Two objects were placed at symmetrical positions on the left and right side of the arena, the center of the object was positioned $24 \mathrm{~cm}$ from the arena wall. Four different sets of objects were used. Each set consisted of 3 identical copies. The different objects were: (1) a standard $1 \mathrm{~L}$ transparent glass bottle (diameter $10 \mathrm{~cm}$, height $22 \mathrm{~cm}$ ) filled with water, (2) a metal cube $(5 \mathrm{~cm} \times 7.5 \mathrm{~cm} \times 10 \mathrm{~cm})$ with two tunnels (diameter $1.5 \mathrm{~cm}$ ) drilled across, (3) a cone (height $16 \mathrm{~cm}$, diameter at base 18 $\mathrm{cm}$ ) consisting of a gray polyvinyl chloride base with a collar on top made of aluminum (diameter $5.0 \mathrm{~cm}$ ) and (4) a solid, 'bullet-shaped' aluminum cube with a tapering top $(8 \mathrm{~cm} \times 8 \mathrm{~cm} \times 13 \mathrm{~cm})$ and a groove near the bottom. The four sets of objects were rotated over the test sessions, using the novel object from the previous session as familiar object in the subsequent session. Because we only used 4 sets of objects the objects were re-used after the 4th test session. So from the 4th test session on, the novel object was always the same object as the familiar object from 3 test sessions before. Rats were unable to displace the objects, a picture of each object is shown in Figure 1. 


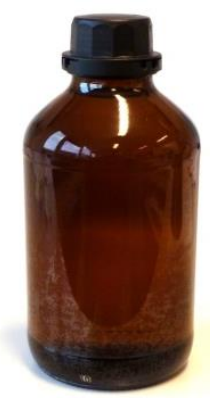

Object 1

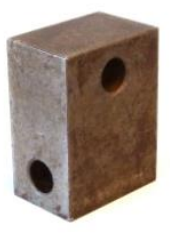

Object 2

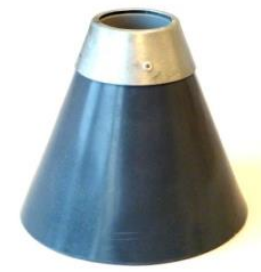

Object 3

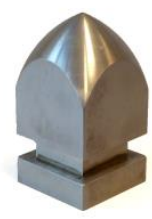

Object 4

Figure 1 Standard object sets used in the ORT

The different objects used in the ORT were; 1) a bottle; 2) a cube; 3) a cone and 4) a bullet shaped piece of aluminum. Rats were unable to displace the objects.

\section{EXPERIMENTAL PROCEDURE AND TREATMENT CONDITIONS}

An ORT session consisted of a sample (T1) and a test trial (T2), of 3 min each, separated by a retention interval of $1 \mathrm{~h}$ or $24 \mathrm{~h}$. At the start of each trial animals were placed in front of the objects, facing the wall. During T1 animals were confronted with two identical sample objects, a1 and a2. In 12 these were replaced by a 'fresh' sample object, a3, and a novel object, object b. The objects were cleansed before each trial with a damp cloth containing a $70 \%$ ethanol solution. During a trial, animals could freely explore the arena and objects, the time spent interacting with each individual object during T1 and T2 was recorded live on a personal computer. Six experienced observers contributed to the current dataset. We have previously shown that experienced observers achieve very high levels of concordance on both exploration and discrimination parameters of the ORT (Rutten, Reneerkens, et al., 2008).

Before experimental testing, the animals need to be habituated to the apparatus and the testing procedures. Although familiarization with the apparatus is known to affect object interaction and possibly discrimination (Besheer \& Bevins, 2000), there is no apparent consensus about what should be involved in ORT familiarization. 
Table 1 Familiarization protocols

\begin{tabular}{|c|c|c|c|c|c|c|c|c|}
\hline $\begin{array}{c}\text { Familiarization } \\
\text { protocol }\end{array}$ & $\begin{array}{l}\text { Empty } \\
\text { arena }\end{array}$ & $\begin{array}{l}\text { Empty } \\
\text { arena }\end{array}$ & $\begin{array}{c}\text { 1st } \\
\text { session }\end{array}$ & $\begin{array}{c}\text { 2nd } \\
\text { session }\end{array}$ & Inj. & $\begin{array}{c}\text { 3rd } \\
\text { session }\end{array}$ & $\begin{array}{c}\text { 4th } \\
\text { session }\end{array}$ & $\begin{array}{c}\geq 5 \text { th } \\
\text { session }\end{array}$ \\
\hline $1 \mathrm{~h}$ & $3 \min$ & $3 \mathrm{~min}$ & $\begin{array}{c}1 \mathrm{~h} \\
\text { interval }\end{array}$ & $\begin{array}{c}24 \mathrm{~h} \\
\text { interval }\end{array}$ & $\begin{array}{l}\text { saline } \\
\text { IP/PO }\end{array}$ & $\begin{array}{l}1 \text { h interval } \\
\text { + saline inj. }\end{array}$ & $\begin{array}{r}1 \mathrm{~h} \text { interval } \\
+ \text { vehicle inj. }\end{array}$ & $\begin{array}{l}1 \mathrm{~h} \text { interval } \\
+ \text { vehicle inj. }\end{array}$ \\
\hline $24 \mathrm{~h}$ & $3 \min$ & $3 \min$ & $\begin{array}{c}1 \mathrm{~h} \\
\text { interval }\end{array}$ & $\begin{array}{c}24 \mathrm{~h} \\
\text { interval }\end{array}$ & $\begin{array}{l}\text { saline } \\
\text { IP/PO }\end{array}$ & $\begin{array}{l}24 \mathrm{~h} \text { interval } \\
+ \text { saline inj. }\end{array}$ & $\begin{array}{l}24 \mathrm{~h} \text { interval } \\
+ \text { vehicle inj. }\end{array}$ & $\begin{array}{l}24 \mathrm{~h} \text { interval } \\
+ \text { vehicle inj. }\end{array}$ \\
\hline
\end{tabular}

The $1 \mathrm{~h}$ and $24 \mathrm{~h}$ familiarization protocols are initially the same, as indicated by the dotted line. Starting with $3 \mathrm{~min}$ in the empty arena for 2 consecutive days, followed by a $1 \mathrm{~h}$ retention test session and a $24 \mathrm{~h}$ retention test session without any injections. After this animals were familiarized the injection procedures by one or more saline injections, using the route and volume required by the subsequent experiment. After this the protocols diverge, with only $1 \mathrm{~h}$ test sessions being performed in the $1 \mathrm{~h}$ familiarization protocol and only $24 \mathrm{~h}$ test sessions in the $24 \mathrm{~h}$ familiarization protocol. From the $3 \mathrm{rd}$ session on, the full experimental procedure is applied and from the 4th session on saline injections are replaced by vehicle injections. Familiarization is completed when animals show no more object discrimination ( $24 \mathrm{~h}$ protocol) or good object discrimination (1 h protocol).

In our lab two familiarization protocols are used, depending on the type of retention delay which will be used in the subsequent experiment. We use a '1 $\mathrm{h}$ familiarization protocol' to prepare animals for $1 \mathrm{~h}$ retention interval testing in combination with drug-induced forgetting. However, when animals are prepared for testing of natural forgetting during a $24 \mathrm{~h}$ retention interval we use a '24 h familiarization protocol', which is a slightly adapted version of the '1 $\mathrm{h}$ familiarization protocol'.

In both protocols (schematically presented in Table 1), familiarization starts with a 3 min habituation trial in the empty arena on 2 consecutive days, in order to let the animals get accustomed to the test environment. After this we start with the 1st familiarization session, a test session with a $1 \mathrm{~h}$ retention interval in which the animals are not subjected to any injections. This is followed by the 2nd familiarization session, a $24 \mathrm{~h}$ retention interval test, also without injecting the animals. These first 2 sessions combined with the first 2 habituation trials give the animals sufficient opportunity to get accustomed to handling and ORT test procedures. On a subsequent day, animals are familiarized to the administration procedure via saline injections, without submitting the animals to ORT testing. These can be one or more injections, intra peritoneal (IP) and/or oral (PO), depending on the demands of the subsequent experiment. Usually, we observe that the animals still discriminate between the novel and old object in the 2nd familiarization session. Therefore, in '24 h familiarization protocol', we continue with $24 \mathrm{~h}$ retention intervals, until the animals show no 
discrimination anymore. This is different from the ' $1 \mathrm{~h}$ familiarization protocol', which is completed by subjecting the animals to a final $1 \mathrm{~h}$ retention interval in the 3rd familiarization session.

In both protocols, training beyond the 2 nd session always includes saline injections at the same time point, volume and via the same route as will be used during the subsequent experiment. This is done to check whether the animals still perform properly when the ORT procedures are applied in combination with the injections. Usually, the familiarization data are not reported in articles because no scientific hypotheses were tested. However, we have included the familiarization data in the current dataset, to investigate the effects of training on exploration and discrimination.

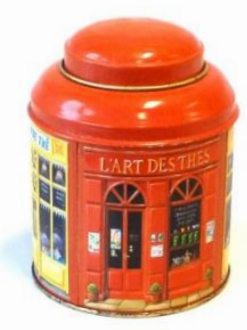

New Object 1

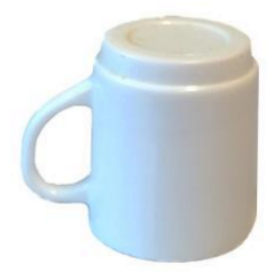

New Object 2

\section{Figure 2 New object sets used in supplementary experiment}

Two completely novel sets of objects were introduced to test the relativity of novelty. Object set 1 consisted of red tin cans and set 2 contained white porcelain cups.

A supplementary experiment was performed to investigate whether the object discrimination we observed in the 2nd familiarization sessions is due to the absolute novelty of the novel object, i.e. animals encounter the novel object for the first time in their life. Data from this experiment was not included in the other analyses in this article, because different object sets were used. 24 fully familiarized animals were subjected to a $24 \mathrm{~h}$ retention interval in the usual test setup that has been described above. However, we used 2 new sets of objects that were completely novel to the animals as novel and familiar object (Figure 2). The objects in the sets were red tin cans (diameter $9 \mathrm{~cm}$, height $11.5 \mathrm{~cm}$ ) and white porcelain cups (diameter $7.3 \mathrm{~cm}$, 
height $9 \mathrm{~cm}$ ). Animals were tested twice and in the 2 nd test session, $96 \mathrm{~h}$ after the $1 \mathrm{st}$ test session, the animals received the exact same objects in the same locations as in the 1 st test session.

The dataset contained 3771 individual ORT sessions divided over conditions which were based on the retention interval used ( $1 \mathrm{~h}$ or $24 \mathrm{~h}$ ), pharmacological treatment and whether the data was collected during or after familiarization, which will be referred to as 'non-familiarized' and 'familiarized' animals, respectively.

Four different treatment groups with a $1 \mathrm{~h}$ retention delay were included in the dataset. First, animals that did not receive any kind of treatment were labeled the ' $1 \mathrm{~h}$ untreated' group. Second, the '1 $\mathrm{h}$ saline' group contained animals that received 1 $\mathrm{ml} / \mathrm{kg}$ saline IP, 30 min before T1. In addition, these animals received an injection with the vehicle of the experimental drug. Because the dataset was composed of animals from different experiments, these additional vehicle injections vary in composition (saline, 1-20\% pluronic solution with tylose $0.5-3 \%$, saline with $2 \%$ tween 80 , tylose solutions of $0.5-5 \%$ with $2 \%$ tween 80 , and $1 \%$ tylose with $5 \%$ mannitol), volume $(1-10 \mathrm{ml} / \mathrm{kg}$ ), timing ( $2 \mathrm{~h}$ or $0.5 \mathrm{~h}$ before T1, or $3 \mathrm{~h}$ after T1), and administration route (IP or PO.). Third, the 'scopolamine' group was treated identical to the 'saline' group, only now the $1 \mathrm{ml} / \mathrm{kg}$ saline injection contained scopolamine, dissolved in a concentration of $0.1 \mathrm{mg} / \mathrm{ml}$. Finally, the 'MK-801_ group received the same treatment as the scopolamine group, but now a concentration of $0.125 \mathrm{mg} / \mathrm{ml}$ MK-801 was dissolved in the $1 \mathrm{ml} / \mathrm{kg}$ saline injection. So, the $1 \mathrm{~h}$ untreated group did not receive any injections. All other $1 \mathrm{~h}$ groups received a vehicle injection in addition to a saline, scopolamine, or MK-801 injection.

In the remaining experiments, pharmacological agents that are considered to facilitate memory were administered to subjects in order to assess their ability to counteract the natural decline of novel object preference that is seen in untreated subjects over a 24 $\mathrm{h}$ retention delay. From these experiments, only the conditions in which the animals received no pharmacological treatment, i.e. untreated and vehicle treated animals were included in the dataset. Together with the familiarization data from the $24 \mathrm{~h}$ retention experiments these sessions will be referred to as the ' $24 \mathrm{~h}$ ' group. The 
vehicles used varied between experiments, for details, see the vehicles described for 1 $\mathrm{h}$ retention experiments. Administration route was either IP or PO and injections were always given after T1 (0 min-3 h). Compositions are amongst the vehicles described for the $1 \mathrm{~h}$ retention studies. To summarize, animals in $24 \mathrm{~h}$ experiments either received a single vehicle injection after $\mathrm{T} 1$, or did not receive any injection.

\section{STATISTICAL ANALYSIS}

The basic output measures in the ORT are the times spent by rats in exploring each object during T1 and T2. From these basic output measures, several variables can be calculated (see Table 2). e1 and e2 are measures of the total time spent investigating both objects during $\mathrm{T} 1$ and $\mathrm{T} 2$, respectively. The $d 1$ index depicts the absolute difference between the sample and the novel object. The $d 2$ index is a relative measure of discrimination corrected for the level of exploration in the test-trial (e2) and the $d 3$ index shows the proportion of e2 devoted to the novel object.

Table 2 Measures in the ORT

\begin{tabular}{cl}
\hline Exploration & Discrimination \\
\hline$e 1=a_{1}+a_{2}$ & $d 1=b-a_{3}$ \\
& $d 2=d 1 / e 2$ \\
$e 2=a_{3}+b$ & $d 3=b / e 2$ \\
\hline
\end{tabular}

The output variables $e 1, e 2, d 1, d 2$ and $d 3$ can be calculated from the time spent exploring both identical objects (a1 and a2) in the first trial (T1), and the time spent in exploring the familiar (a3) and novel (b) object in the second trial (T2).

One-sample $t$-statistics were performed to assess whether the $d 1$ and $d 2$ indices were different from zero, since random exploration in T2 would result in equal exploration of both objects. The $d 3$ index was compared to 0.5 because if both objects in $T 2$ are equally explored, the proportion of exploration directed towards the novel objects will be $50 \%$. Between group effects on e1, e2 and the $d$ measures were also assessed using one-way ANOVA. In case of significant differences, pair-wise comparisons were performed using Bonferroni correction. The relation between the different output measures was investigated by calculating their Pearson correlation coefficient 


\section{RESULTS}

\section{FAMILIARIZATION EFFECTS}

\section{H FAMILIARIZATION PROTOCOL}

The $1 \mathrm{~h}$ familiarization protocol involves 3 test sessions. The '1st session' $(n=443)$, which is a $1 \mathrm{~h}$ retention interval. The ' $2 \mathrm{nd}$ session' $(\mathrm{n}=444)$, a $24 \mathrm{~h}$ retention interval. Familiarization is completed after the '3rd session', a 1 h retention interval $(n=336)$ with saline injection(s). The '4th session' was also included separately $(n=96)$. All subsequent $1 \mathrm{~h}$ saline test sessions were combined and will be referred to as the ' 5 th session' ( $n=901$ ). These test sessions only included data from vehicle treated animals.

The development of e1 and e2 is depicted in Figure 3. Oneway ANOVA showed that e1 and e2 were significantly different over the course of familiarization, $(F(4,2215)=$ 101.78, $p<0.001)$ and $(F(4,2215)=11.55, p<0.001)$, respectively. Individual sessions were compared via post hoc analyses with Bonferroni correction. This revealed that, in the 1st session, e1 was higher than in all subsequent sessions $(p<$ 0.001). Furthermore, e1 in the 2 nd session was significantly higher, compared to the 5 th session $(p<0.001)$. e2 proved to be not significantly different in the $1 \mathrm{st}, 2 \mathrm{nd}$ and 5th sessions $(p>0.05)$. In the 3rd session, e2 was higher than in all other sessions ( $p$ $<0.05)$. The 4 th session showed a lower e2 compared to all other sessions $(p<0.01)$, except for the 5th session, which had a statistically equal e2 $(p>0.05)$.

Paired-samples $t$-tests were performed on e1 and e2 within each session, the results of which are displayed in Figure 3. The $d 1$ values of the successive sessions are shown in Figure 4A. Using one-sample $t$-tests, the $d 1$ was found to be significantly higher than zero in all of the sessions. ANOVA revealed that the $d 1$ values of the familiarization sessions were significantly different $(F(4,2215)=54.39, p<0.001)$. Post hoc comparison revealed no significant differences between the 1st, $4 \mathrm{rd}$ and 5 th session $(p>0.05)$, whereas $d 1$ in the 2 nd session was lower $(p<0.001)$ and $d 1$ in the 3rd session was higher $(p<0.001)$ compared to all other sessions. 


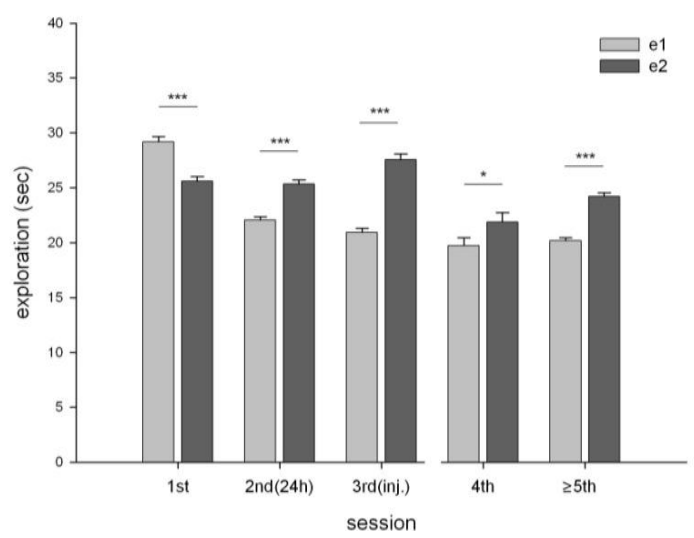

\section{Figure $31 \mathrm{~h}$ familiarization protocol - exploration}

The development of $e 1$ and $e 2$ in the $1 \mathrm{~h}$ familiarization protocol. The $x$-axis shows the familiarization sessions in chronological order, additional information about particular sessions is specified within brackets. All sessions used $1 \mathrm{~h}$ retention intervals, except for the 2 nd session, in which a $24 \mathrm{~h}$ retention interval was used. With the exception of the 3 rd session, animals did not receive injections during familiarization (session $1-3$ ), during testing (4th and $\geq 5$ th session) animals did receive injections. The 4 th session represents the first $1 \mathrm{~h}$ saline test sessions after familiarization, session $\geq 5$ is a collection of the remainder of all $1 \mathrm{~h}$ saline test sessions. A difference between $\mathrm{e} 1$ and $e 2$ is indicted with asterisks (paired samples t-test, * $p<0.05 ;{ }^{* * *} p$ $<0.001)$.
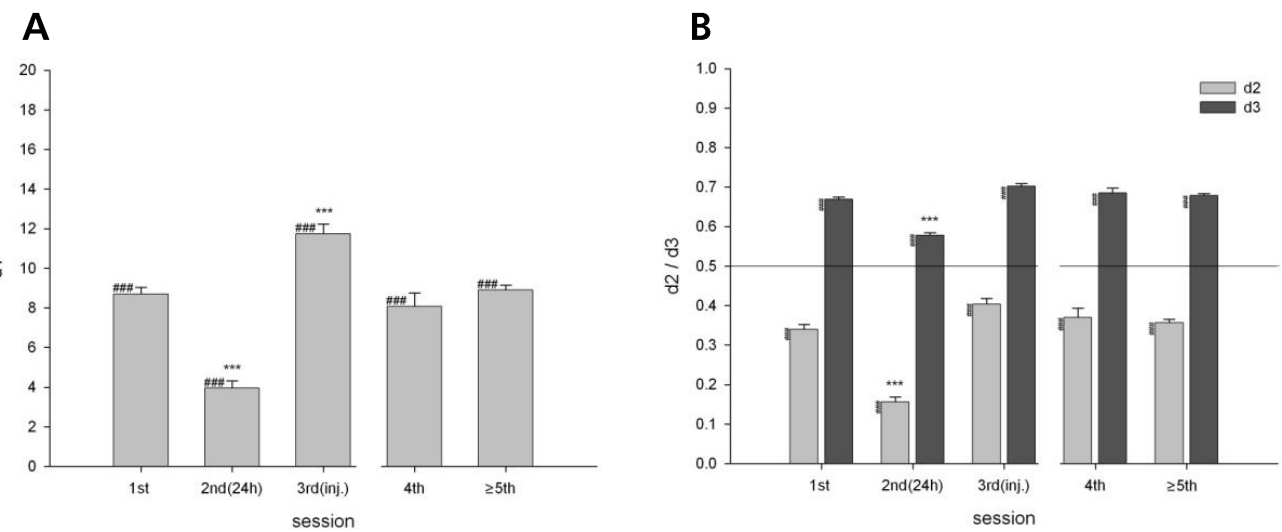

\section{Figure $41 \mathrm{~h}$ familiarization protocol - discrimination}

The $d 1$ measure $(\mathbf{A}), d 2$ and $d 3$ measures (B) of the different familiarization sessions in the $1 \mathrm{~h}$ familiarization protocol. The $x$-axis shows the familiarization sessions in chronological order, additional information about particular sessions is specified within brackets. All sessions used $1 \mathrm{~h}$ retention intervals, except for the 2nd session, in which a $24 \mathrm{~h}$ retention interval was used. With the exception of the $3 \mathrm{rd}$ session, animals did not receive injections during familiarization (sessions 1-3), during testing (4th and $\geq 5$ th session) animals did receive injections. The 4 th session represents the first $1 \mathrm{~h}$ saline test sessions after familiarization, session $\geq 5$ is a collection of the remaining $1 \mathrm{~h}$ saline test sessions. A difference from the $1 \mathrm{st}$ familiarization session is indicated with asterisks (Bonferroni t-tests, $* * * p<0.001$ ). Significant differences from zero are indicated with hashes (one-sample t-tests, \#\#\# $p<0.001$ ). 
One-sample $t$-tests showed that all conditions in the $1 \mathrm{~h}$ familiarization protocol had $d 2$ and $d 3$ values that were significantly higher than 0 and 0.5 , respectively (Figure 4B). Like $d 1$, ANOVA showed that the $d 2$ and $d 3$ measures were significantly different between sessions; $(F(4,2215)=62.13, p<0.001)$ for $d 2$ and $(F(4,2215)=61.94, p<$ $0.001)$ for $d 3$. Bonferroni $t$-tests showed that the $d 2$ and $d 3$ values in the 2 nd session were significantly lower compared to all of the other sessions $(p<0.001)$. This is in contrast with the 3rd session, in which both were significantly higher than all the other sessions $(p<0.01)$, except the 4 th $(p>0.05)$. No further differences were found.

\section{H FAMILIARIZATION PROTOCOL}

The first two sessions in the $24 \mathrm{~h}$ familiarization protocol were the same sessions as those in the $1 \mathrm{~h}$ familiarization protocol. The protocols diverge after the $2 \mathrm{nd}$ familiarization session. Hence, in both protocols the same data was used for the analyses of the $1^{\text {st }}$ and 2 nd session. In the $24 \mathrm{~h}$ familiarization protocol, the ' $3 \mathrm{rd}$ session' was a $24 \mathrm{~h}$ interval $(n=96)$ with saline injection(s), after this the experiments started. The '4th session', which is the first $24 \mathrm{~h}$ vehicle test session, was also included $(n=48)$ in the analyses and the remaining $24 \mathrm{~h}$ vehicle test sessions were combined in the '5th session' ( $n=267$ ). The e1, e2, $d 1, d 2$ and $d 3$ measures of the different sessions are graphically presented in Figures 5 and 6 .

In Figure 5, e1 and e2 are shown for each session. ANOVA demonstrated that exploration varied significantly between the different sessions, e1 $(F(4,1293)=69.76$, $p<0.001)$ and e2 $(F(4,1293)=24.29, p<0.001)$. Post hoc analysis with Bonferroni correction showed that, compared to all other sessions, e1 was higher in the 1st session $(p<0.001)$ and lower in the 4 th session $(p<0.001)$. No e1 differences were found between the 2 nd, $3 r d$ and 5 th session $(p>0.05)$. The e2 value of the $3 r d$ session was higher than that of the other sessions $(p<0.05)$, whereas e2 in the 4 th session was lower $(p<0.001)$. Furthermore, e2 in the 5 th session was lower than in the 1 st and 2 nd session $(p<0.05$ ). No difference was found between the e2 measure of the 1 st and 2 nd session ( $p>0.05$ ). 


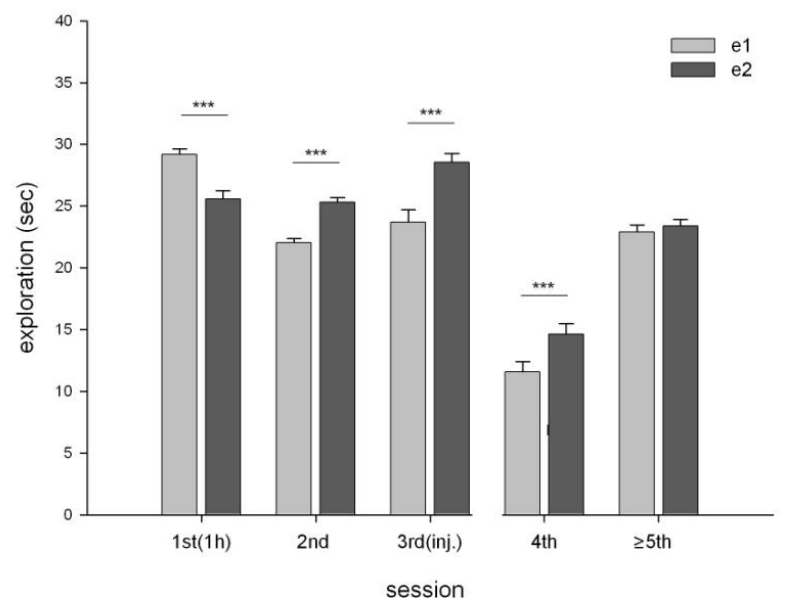

Figure $524 \mathrm{~h}$ familiarization protocol - exploration

The development of $e 1$ and $e 2$ in the $24 \mathrm{~h}$ familiarization protocol. The $x$-axis shows the familiarization sessions in chronological order, additional information about particular sessions is specified within brackets. All sessions used $24 \mathrm{~h}$ retention intervals, except the 1 st session, in which a $1 \mathrm{~h}$ retention interval was used. With the exception of the 3rd session, animals did not receive injections during familiarization (sessions 13 ), during testing (4th and $\geq 5$ th session) animals did receive injections. The 4 th session represents the first $24 \mathrm{~h}$ vehicle test sessions after familiarization, session $\geq 5$ is a collection of the remaining $24 \mathrm{~h}$ vehicle test sessions. A difference between $e 1$ and $e 2$ is indicted with asterisks (paired-samples t-test, $* * * p<0.001$ ). Of note, because the familiarization protocols are identical up to the 3 rd session, the 1st and the 2 nd familiarization sessions in the $24 \mathrm{~h}$ familiarization protocol are the same as those in the $1 \mathrm{~h}$ familiarization protocol.

Within each individual session, e1 and e2 were compared using paired-samples $t$ tests. It was found that e2 was higher than $e 1$ in the 2 nd, $3 r d$ and 4 th sessions ( $t(443)$ =9.22, $p<0.001),(t(95)=5.37, p<0.001)$ and $(t(47)=3.56, p<0.001)$, respectively. On the other hand, in the 1st session e2 was lower than e1 $(t(442)=$ $8.37, p<0.001)$ and in the 5 th session no statistically significant difference was found $(t(266)=1.01$, n.s. $)$.

The mean $d 1$ values of the $24 \mathrm{~h}$ familiarization protocol and subsequent test sessions are graphically presented in Figure 6A. One-way ANOVA showed that $d 1$ values are significantly different between sessions $(F(3,728)=3.50, p<0.05)$. Post hoc analysis was performed using Bonferroni correction. The $d 1$ value was shown to be higher in the 1st session, compared to the rest of the sessions $(p<0.001)$. Additionally, the 2 nd session was found to have a higher $d 1$ than the 4 th and 5 th sessions $(p<0.05)$. Finally, $d 1$ was higher in the 3rd session compared to the 5th session $(p<0.01)$. 
A

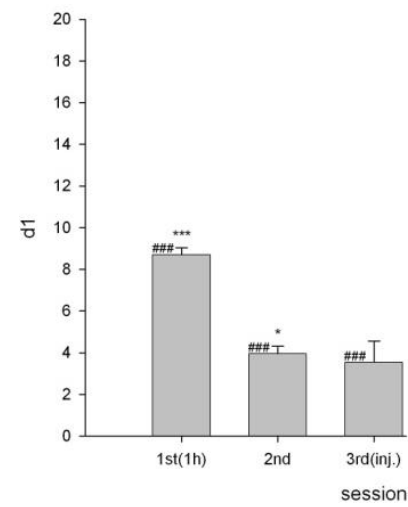

B

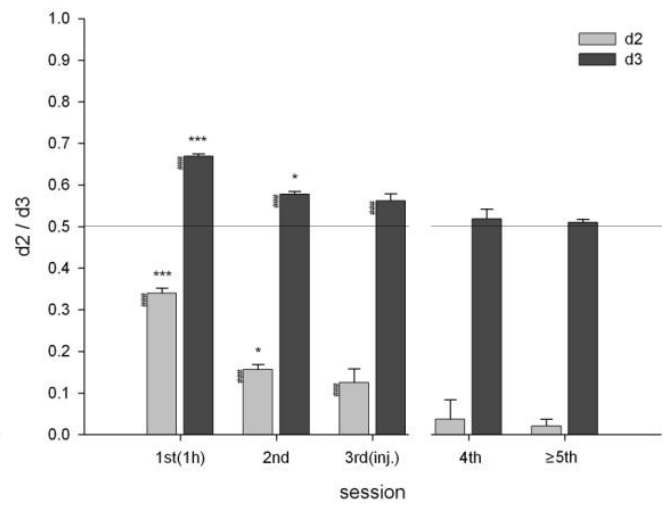

Figure $624 \mathrm{~h}$ familiarization protocol - discrimination

The $d 1$ measure (A), $d 2$ and $d 3$ measures (B) of the different familiarization sessions in the $24 \mathrm{~h}$ familiarization protocol. All sessions used $24 \mathrm{~h}$ retention intervals, except the $1 \mathrm{st}$ session, in which a $1 \mathrm{~h}$ retention interval was used. With the exception of the 3rd session, animals did not receive injections during familiarization (sessions 1-3), during testing (4th and $\geq 5$ th session) animals did receive injections. The 4 th session represents the first $24 \mathrm{~h}$ vehicle test sessions after familiarization, session $\geq 5$ is a collection of the remaining $24 \mathrm{~h}$ vehicle test sessions. A difference from the 4 th session (first test session) is indicated with asterisks (Bonferroni $t$-tests, ${ }^{*} p<0.05 ;{ }^{* *} p<0.001$ ). Significant differences from zero are indicated with

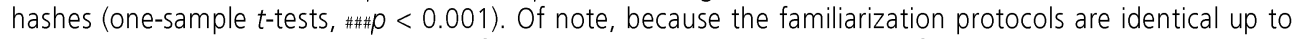
the $3 \mathrm{rd}$ session, the 1 st and the 2 nd familiarization sessions in the $24 \mathrm{~h}$ familiarization protocol are the same as those in the $1 \mathrm{~h}$ familiarization protocol.

The $d 2$ and $d 3$ measures of the $24 \mathrm{~h}$ familiarization sessions are shown in Figure 6B. ANOVA showed significant differences between familiarization sessions on $d 2$ $(F(4,1293)=68.25, p<0.001)$ and $d 3(F(4,1293)=67.56, p<0.001)$. Bonferroni $t$ tests revealed that $d 2$ and $d 3$ were higher in the 1 st session, compared to all subsequent sessions $(p<0.001)$. Furthermore, both measures were found to be higher in the 2 nd session, compared to the 4 th and 5 th session $(p<0.05)$. Finally, the $3 r d$ session was found to have higher $d 2$ and $d 3$ values compared to the 5 th session $(p<0.05)$.

\section{NOVELTY}

In our experimental ORT setup, 4 sets of objects are used. These 4 sets are rotated over the animals in such a way that the familiar object from a certain test session serves as novel object 3 test sessions later. For example, if a particular object was used 


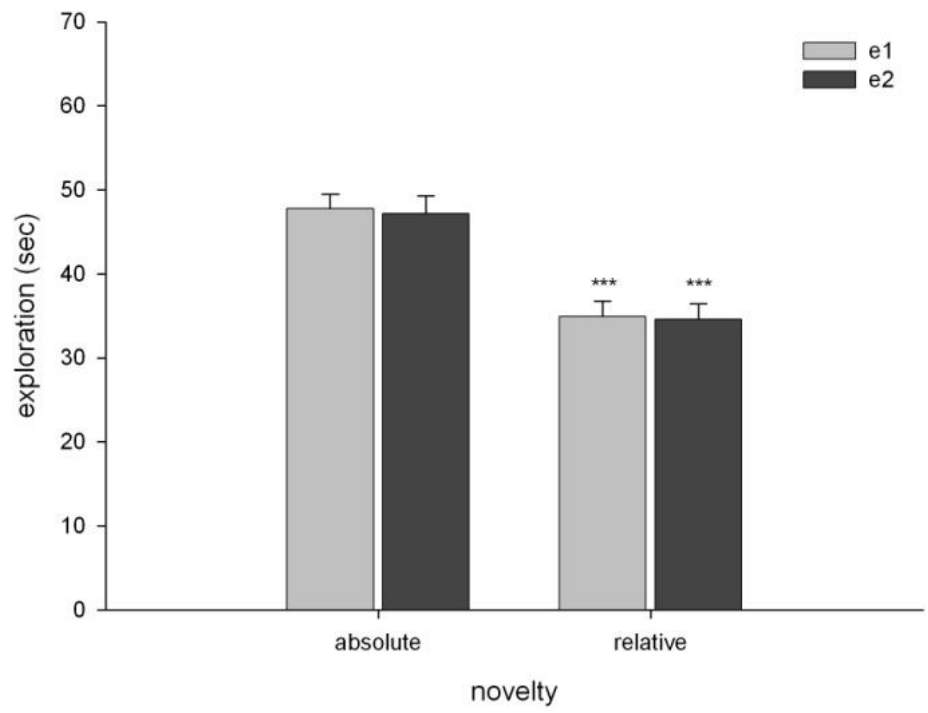

Figure 7 Absolute and relative novelty in the ORT- exploration

Exploration of objects that are absolutely novel or relatively novel. Animals performed two $24 \mathrm{~h}$ retention sessions containing the exact same objects, divided by a 4-day period. The $x$-axis shows the consecutive sessions in which they were confronted with objects they had never encountered before (absolute novelty) and the same objects again (relative novelty). A difference from the 1st session (absolute novelty) is indicated with asterisks (Bonferroni t-tests, ${ }^{* *} p<0.001$ ).
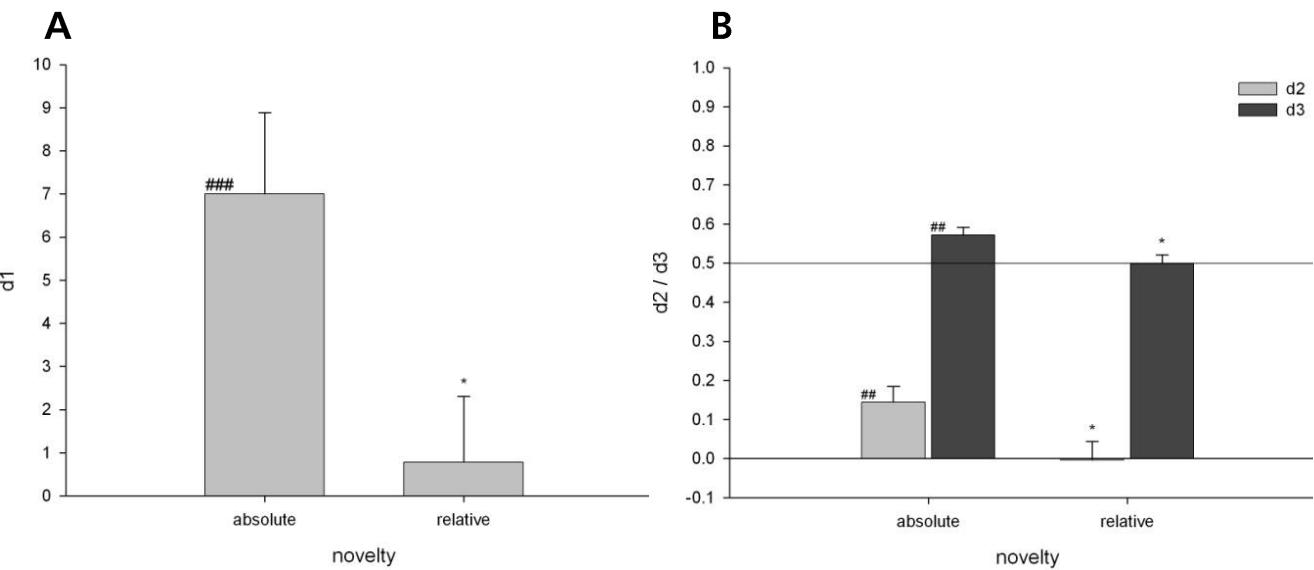

Figure 8 Absolute and relative novelty in the ORT- discrimination

The $d 1(\mathbf{A}), d 2$ and $d 3$ measures $(\mathbf{B})$ of the same objects in two consecutive $24 \mathrm{~h}$ retention sessions. Animals were confronted with objects they had never seen before (absolute novelty) in the 1st session and the same objects again in the 2 nd session (relative novelty). Sessions were separated by a 4-day period. Differences from the 1st session (absolute novelty) are indicated with asterisks (Bonferroni $t$-tests, ${ }^{\star} p<0.05$ ) and significant differences from zero are indicated with hashes (one-sample t-tests, \#\# $<0.01 ; \# \# p<0.001$ ). 
animals only do not discriminate between objects anymore (in a $24 \mathrm{~h}$ retention interval) in the 4 th test session. To investigate whether this phenomenon was related to the re-use of the object-sets we performed a supplementary experiment in which animals $(n=24)$ performed two consecutive $24 \mathrm{~h}$ retention sessions. These animals had already encountered all 4 sets of standard objects and showed no discrimination in a $24 \mathrm{~h}$ retention interval. In the $24 \mathrm{~h}$ test session of this experiment two completely new sets of objects were used, meaning that the animals had never before encountered these objects. The new objects are shown in Figure 2. In the 1st session the novel object encountered by the animals in T2 was absolutely novel (i.e. never before encountered) to the animals. The 2 nd $24 \mathrm{~h}$ test session was 4 days later and exactly identical to the 1 st session for each individual animal, meaning that the exact same objects were presented as being familiar or novel. Thus, in the 2nd test session the novel object is not novel anymore in an absolute sense, but it is novel relative to the familiar object in that particular test session. The exploration measures of the 1st and 2 nd test session are presented in Figure 7 and discrimination measures are graphically presented in Figure 8.

\section{TREATMENT EFFECTS OF MK-801 AND SCOPOLAMINE}

To assess the effect of different treatment conditions on behavior in the ORT, only familiarized animals were incorporated. The data used in the analysis of the treatment effects are graphically presented in Figures 9 and 10 .

Comparison of the different treatment conditions with ANOVA revealed that there were differences in e1 $(F(3,1678)=7.94, p<0.001)$ and e2 measures $(F(3,1678)=$ $9.61, p<0.001)$. Post hoc analysis showed that in T1, untreated animals explored less compared to saline $(1 \mathrm{~h})$ treated animals $(p<0.001)$. In T2 no differences between these groups were found. Also, in comparison with saline (1 h) treated animals, e2 was lower in scopolamine treated animals $(p<0.001)$. Furthermore, paired-samples $t$ tests pointed out that e2 was increased compared to e1 in all $1 \mathrm{~h}$ intervals. The effects of the different treatments on exploration are visualized in Figure 9. 


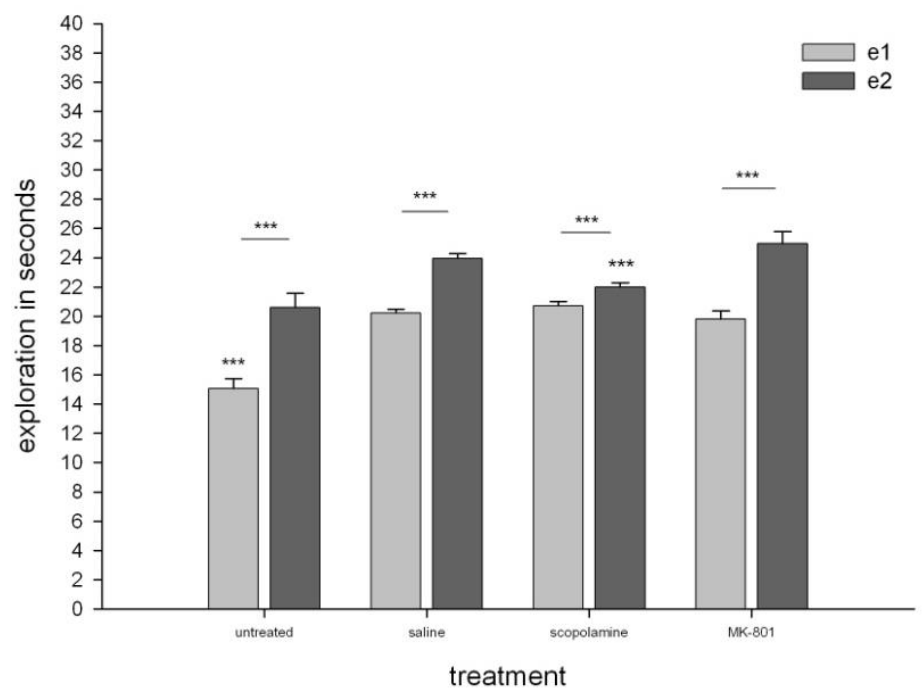

Figure 9 Amnesic treatments - exploration

Effects of different treatments on exploratory behavior in T1 (e1) and T2 (e2) in a $1 \mathrm{~h}$ retention interval. The $x$-axis shows the different treatment conditions and the inter-trial interval. Differences from the saline $(1 \mathrm{~h})$ condition are depicted with asterisks above the error bars (Bonferroni $t$-tests, ${ }^{*} p<0.05 ;{ }^{*} *{ }^{*} p<0.001$ ), underlined asterisks indicate a difference between e1 and e2 within the treatment conditions (pairedsamples $t$-tests, $\left.{ }^{* * *} p<0.001\right)$.

A

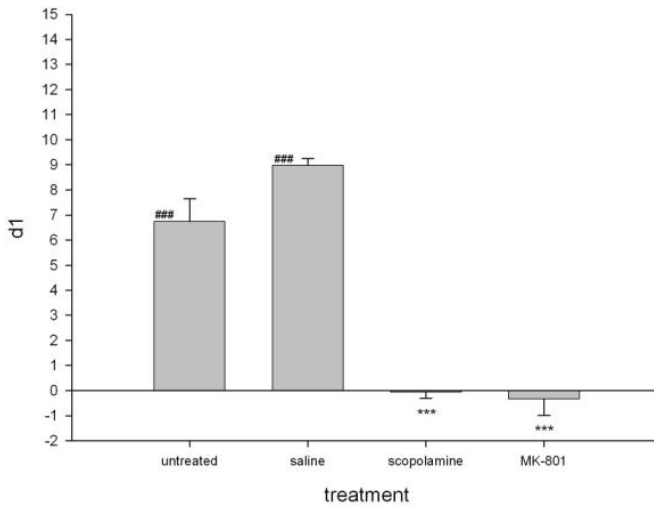

B

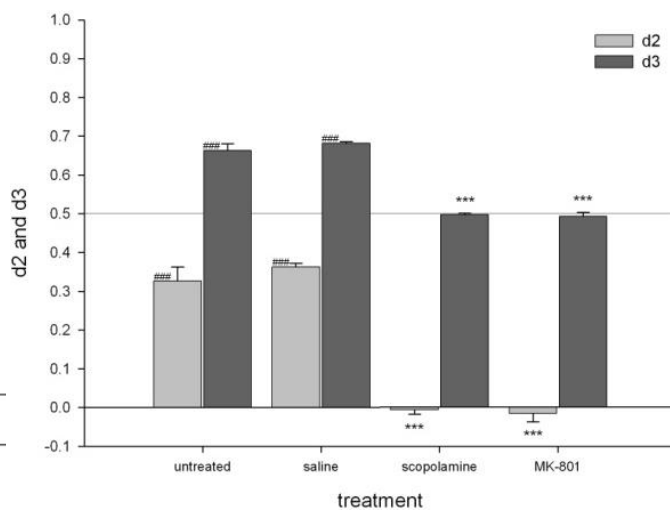

Figure 10 Amnesic treatments - discrimination

The effects of the different treatments on $d 1$ (A) and $d 2 / d 3$ (B) in a $1 \mathrm{~h}$ retention interval. Different

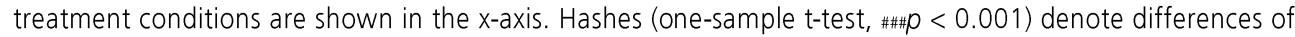
$d 1$ and $d 2$ from 0 , or 0.5 in case of $d 3$. Significant differences from the saline $(1 \mathrm{~h})$ treated group are indicated by asterisks (Bonferroni t-tests, $* * * p<0.001$ ). 
The effects of the different treatments on discrimination are depicted in Figure 10. Significant differences were found on the $d 1(F(3,1678)=222.64, p<0.001)$, $d 2$ $(F(3,1678)=266.29, p<0.001)$ and $d 3$ measures $(F(3,1678)=266.29, p<0.001)$. Post hoc analysis revealed no significant differences $(p>0.05)$ between the untreated animals and animals in the saline $(1 \mathrm{~h})$ condition on any of the discrimination measures. Also, saline ( $1 \mathrm{~h}$ retention interval) treated animals showed significantly more discrimination than animals treated with scopolamine or MK-801 $(p<0.001)$. This was the case for $\mathrm{d} 1$ (Figure 10A), d2 and d3 measures (Figure 10B).

\section{RELATIONSHIPS BETWEEN EXPLORATION AND DISCRIMINATION}

\section{MEASURES}

To investigate the relationship between the level of exploration in the learning and test trial and the three different discrimination measures, only the treatment conditions in which animals discriminated were included. Thus, only sessions from the $1 \mathrm{~h}$ untreated and $1 \mathrm{~h}$ saline conditions were included and overall Pearson's correlations were calculated between e1 and e2, as well as their correlations with $\mathrm{d} 1$, $\mathrm{d} 2$ and $\mathrm{d} 3$. Additionally, the same correlations were analyzed separately for familiarized animals and animals that were not yet fully familiarized to investigate whether there was a difference in this respect between fully familiarized animals and animals that still underwent familiarization (Table 3).

Table 3 Pearsons correlations

\begin{tabular}{lllllllllllllllll}
\hline & $\begin{array}{l}\text { e1 } \\
\text { all }\end{array}$ & tr. & untr. & $\begin{array}{l}\text { e2 } \\
\text { all }\end{array}$ & tr. & untr. & all & tr. & untr. & all & tr. & untr. & $\begin{array}{l}\text { all } \\
\text { tr. }\end{array}$ & untr. \\
\hline e1 & 1 & 1 & 1 & 0.40 & 0.41 & 0.36 & 0.02 & 0.03 & -0.04 & -0.17 & -0.16 & -0.21 & -0.16 & -0.16 & -0.21 \\
sig & & & & 0.000 & 0.000 & 0.000 & n.s. & n.s. & n.s. & 0.000 & 0.000 & 0.000 & 0.000 & 0.000 & 0.000 \\
e2 & 0.40 & 0.41 & 0.36 & 1 & 1 & 1 & 0.56 & 0.57 & 0.54 & 0.12 & 0.12 & 0.10 & 0.12 & 0.12 & 0.10 \\
sig & 0.000 & 0.000 & 0.000 & & & & 0.000 & 0.000 & 0.000 & 0.000 & 0.000 & 0.001 & 0.000 & 0.000 & 0.001 \\
\hline
\end{tabular}

Pearson correlations $(r)$ between $d 1, d 2, d 3, e 1$ and $e 2$. Animals from the untreated and $1 \mathrm{~h}$ saline conditions were pooled together and overall (all, $n=1896$ ) correlations were calculated. Additionally, familiarized (fam., $n=948$ ) and non-familiarized (non-fam., $n=948$ ) animals were analyzed separately. The corresponding significance level is shown below each individual $r$ value.

Cohen (1988) postulated the following guidelines for the interpretation of the correlation coefficient; $r=0.1-0.23$, small effect; $r=0.24-0.36$, medium effect; $r=$ $0.37-1$, large effect. According to these criteria a positive, large overall correlation 


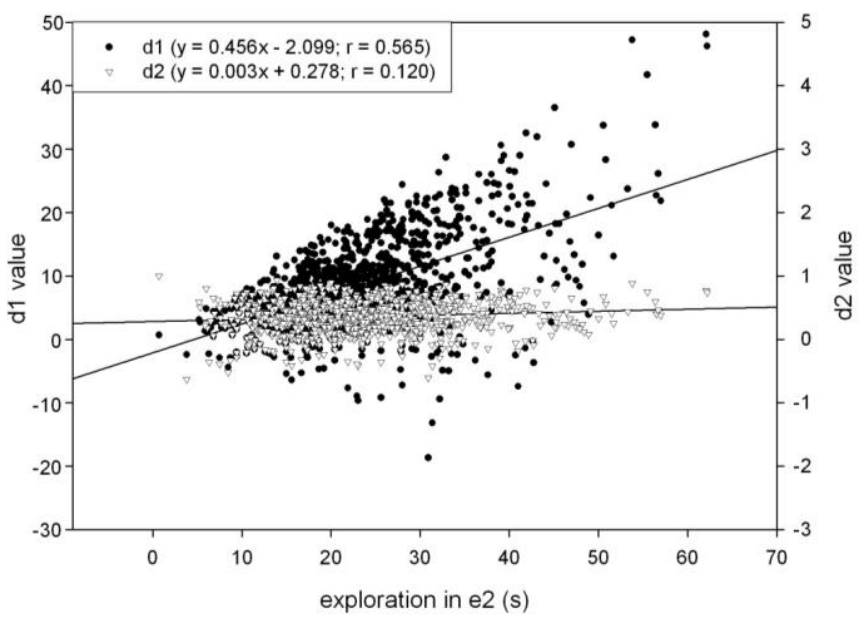

Figure 11 Correlation of exploration and discrimination measures

Relationship between exploration in the test trial (e2) and the $d 1 / d 2$ measures for familiarized and nonfamiliarized animals pooled together, the best fitting straight line is defined by $Y=b X+a$, where $Y=d 1$ or $d 2, X=e 2, b=$ the slope of the line and $a=$ the intercept, and $r=$ the Pearson's correlation coefficient.

was found between e1 and e2. There was no significant correlation between e1 and $d 1$, whereas the negative correlations between e1 and $d 2 / d 3$ were only very low. A large positive overall correlation was found between e2 and $d 1$. Correlations between e2 and $d 2 / d 3$ were also positive, but very low. The same was found when familiarized and non-familiarized animals were separately analyzed (data not shown). The relations between $d 1 / d 2$ and $e 2$ are graphically presented in Figure 11, illustrating the clear relationship of $d 1$ with $e 2$, but not for $d 2$, which corrects for exploratory differences.

Within the familiarized and non-familiarized groups, subdivisions were made based on the total amount of exploration of the individual animals, to investigate the relationship between exploration and discrimination in further detail. For each exploration time bin group, one-sample $t$-tests were performed assessing whether $d 1$ and $d 2$ were significantly higher than zero and whether $d 3$ was different from 0.5 .

Figure $12 \mathrm{~A}$ and $\mathrm{B}$ show the discrimination measures plotted against subsequent time bins of e1 and e2 in familiarized animals, thus showing the relationship between the level and the discrimination measures. Table 4 shows the frequencies per time bin for e1 and e2 of both the familiarized and non-familiarized animals, as well as the 


\section{A}

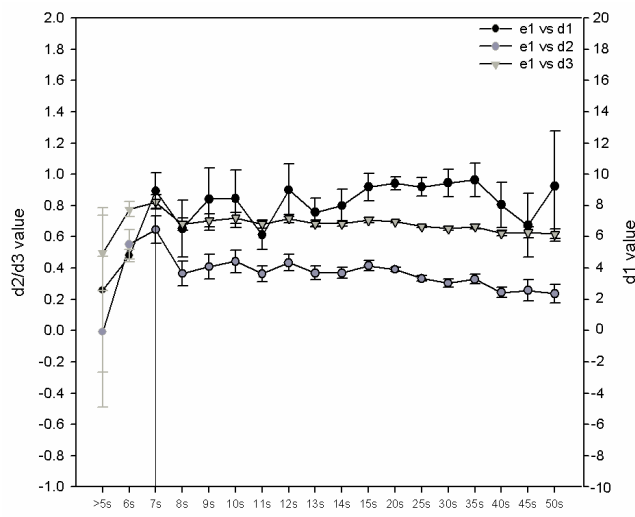

e1

\section{B}

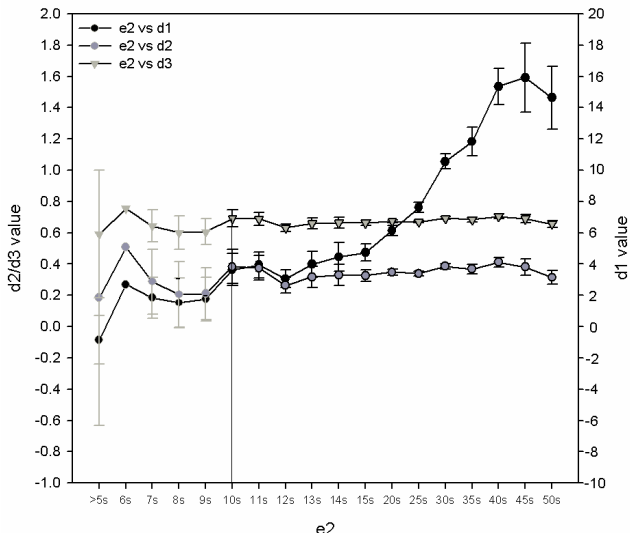

Figure 12 Discrimination measures plotted against exploration level

Exploration in the learning trial $(e 1, \mathbf{A})$ and test trial $(e 2, \mathbf{B})$ plotted against $d 1, d 2$ and $d 3$, for familiarized animals. Subsequent time bins range from the previous value to the maximally shown value. For $d 1$ and $d 2$ groups that significantly differed from 0 (one-sample $t$-test, $p<0.05$ ) are encircled with a black line, when the difference was not significant a grey color was used as outline. The $d 3$ measure was compared to 0.5 and indicated with triangles. For significance indication, the same color coding is used as with the $d 1$ and $d 2$ measures. Vertical black lines show the point from which discrimination remained stable.

minimal amount of exploration that was required for reliable discrimination performance. Meaning that, when exploration further increased the $d 1$ and $d 2$ values remained significantly higher than zero, or 0.5 in case of the $d 3$ measure. It was found that non-familiarized animals required 9-10 s of exploration in T1, whereas

Table 4 Exploration and discrimination frequencies

\begin{tabular}{|c|c|c|c|c|c|c|c|c|c|c|c|c|c|c|c|c|c|c|c|c|}
\hline Exploration (s) & $<5$ & 6 & 7 & 8 & 9 & 10 & 11 & 12 & 13 & 14 & 15 & 20 & 25 & 30 & 35 & 40 & 45 & 50 & $>50$ & Total \\
\hline e1 untrained (n) & 3 & 2 & 3 & 9 & 17 & 18 & 26 & 31 & 34 & 42 & 54 & 260 & 213 & 90 & 50 & 25 & 13 & 7 & 14 & 911 \\
\hline$\%$ of total $n$ & 0,3 & 0,2 & 0,3 & 1,0 & 1,9 & 2,0 & 2,9 & 3,4 & 3,7 & 4,6 & 5,9 & 28,5 & 23,4 & 9,9 & 5,5 & 2,7 & 1,4 & 0,8 & 1,5 & 100 \\
\hline e1 trained (n) & 2 & 2 & 2 & 7 & 9 & 13 & 15 & 12 & 20 & 36 & 32 & 195 & 208 & 184 & 114 & 78 & 40 & 11 & 4 & 984 \\
\hline$\%$ of total $n$ & 0,2 & 0,2 & 0,2 & 0,7 & 0,9 & 1,3 & 1,5 & 1,2 & 2,0 & 3,7 & 3,3 & 19,8 & 21,1 & 18,7 & 11,6 & 7,9 & 4,1 & 1,1 & 0,4 & 100 \\
\hline e2 untrained (n) & 2 & 1 & 5 & 4 & 5 & 8 & 13 & 20 & 17 & 20 & 29 & 189 & 216 & 173 & 96 & 44 & 27 & 18 & 20 & 907 \\
\hline$\%$ of total $n$ & 0,2 & 0,1 & 0,6 & 0,4 & 0,6 & 0,9 & 1,4 & 2,2 & 1,9 & 2,2 & 3,2 & 20,8 & 23,8 & 19,1 & 10,6 & 4,9 & 3,0 & 2,0 & 2,2 & 100 \\
\hline e2 trained (n) & 0 & 3 & 4 & 2 & 6 & 4 & 13 & 17 & 17 & 22 & 21 & 163 & 228 & 185 & 141 & 72 & 55 & 21 & 13 & 987 \\
\hline$\%$ of total $\mathbf{n}$ & 0,0 & 0,3 & 0,4 & 0,2 & 0,6 & 0,4 & 1,3 & 1,7 & 1,7 & 2,2 & 2,1 & 16,5 & 23,1 & 18,7 & 14,3 & 7,3 & 5,6 & 2,1 & 1,3 & 100 \\
\hline
\end{tabular}

Frequencies and percentages of familiarized (fam.) and non-familiarized (non-fam.) animals reaching the criteria for subsequent exploration bins in a $1 \mathrm{~h}$ retention experiments. The total amount of e1 or e2 is depicted in the header row for subsequent time bins, ranging from its own value to that of the prior exploration bin. Threshold exploration values, i.e. minimal exploration required for discrimination to remain significant in subsequent time bins, are indicated by a vertical line. 
familiarized animals only needed 6-7 s of exploration. Both non-familiarized and familiarized animals generally needed more exploration in T2, as compared to T1. In T2, non-familiarized animals also needed more exploration compared to familiarized animals, 11-12 s and 9-10 s, respectively. This means that, in $\mathrm{T} 1,3.7 \%$ of the nonfamiliarized animals and $0.4 \%$ of the familiarized animals did not show sufficient exploration for reliable discrimination performance. In $\mathrm{T} 2,4.2 \%$ of the nonfamiliarized animals and $1.5 \%$ of the familiarized animals did not reach the exploration threshold. Thus, the proportion of animals that showed a sufficient amount of exploration was bigger in the familiarized group.

\section{TRIAL DURATION}

The within-trial development of exploration and discrimination was assessed in familiarized animals using a $1 \mathrm{~h}$ interval $(\mathrm{n}=36)$, the total exploration in T1 and T2 was divided over 6 time bins of $30 \mathrm{~s}$ each. Exploration was distributed over the consecutive time bins as follows: $27.8 \%, 17.5 \%, 18.5 \%, 12.7 \%, 10.8 \%$ and $12.7 \%$ in $\mathrm{T} 1$, and $29.7 \%, 21.8 \%, 16.3 \%, 12.3 \%, 9.9 \%$ and $10 \%$ in T2 (Figure 13B). The cumulative exploration was also calculated after 30 s, 60 s, 90 s, 120 s, 150 s and 180 $s$ of trial duration (Figure 13A). Exploration kept increasing with time, ANOVA showed that the differences between trial durations were significant in both $T 1(F(5,210)=$ 48.65, $p<0.001)$ and $T 2(F(5,210)=44.15, p<0.001)$. The results from Bonferroni post hoc analysis are displayed in Figure 13A.

Development of $d 1, d 2$ and $d 3$ measures was analyzed in the same cumulative fashion as e1 and e2 (Figure 14). The $d$ measures were calculated for the hypothetical situations that the trial ended after $30 \mathrm{~s}, 60 \mathrm{~s}, 90 \mathrm{~s}, 120 \mathrm{~s}, 150 \mathrm{~s}$ and after the actual duration of $180 \mathrm{~s}$. One-sample $t$-tests showed that $d 1$ and $d 2$ measures were significantly higher than zero for all theoretical trial durations. ANOVA revealed significant differences in the $d 1$ measure between trial durations $(F(5,210)=4.49, p<$ $0.001)$, but not in the $d 2(F(5,210)=0.53$, n.s. $)$ and $d 3$ measures $(F(5,210)=0.53$, n.s.) Post hoc analyses were performed using Bonferroni $t$-tests, results are displayed in Figure $14 \mathrm{~A}$ and $\mathrm{B}$. 
A

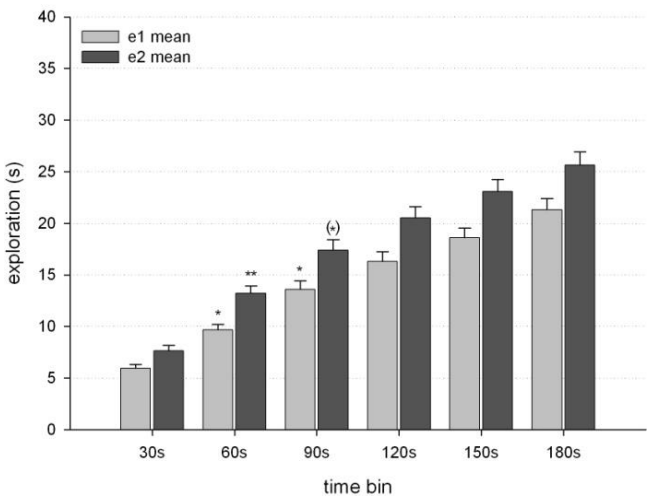

\section{B}

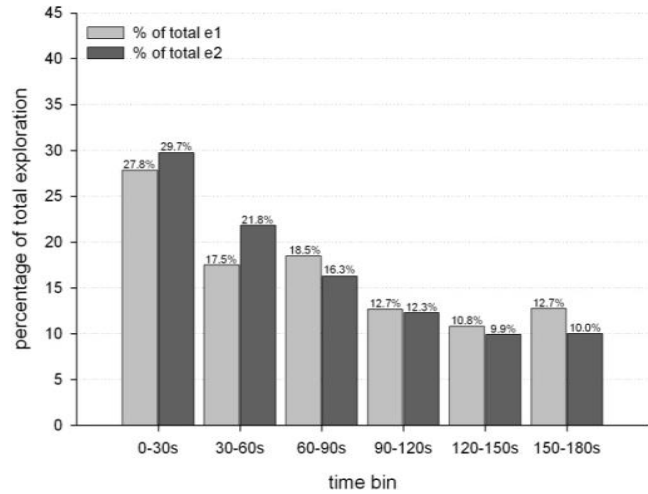

Figure 13 Distribution of exploration within the trials

Distribution of exploration in T1 and T2 presented in time bins of $30 \mathrm{~s}$. Fig. A shows the cumulative exploration, given the hypothetical situation if the trial would have been stopped after the time bin depicted on the $\mathrm{x}$-axis. Fig. B shows the exploration per time bin as a percentage of the total exploration over $180 \mathrm{~s}$. A significant increase from the preceding time bin is indicated by asterisks (Bonferroni $t$-tests, $\left.\left.{ }^{*}\right) p<0.06 ;{ }^{*} p<0.05 ;{ }^{*} p<0.01\right)$.
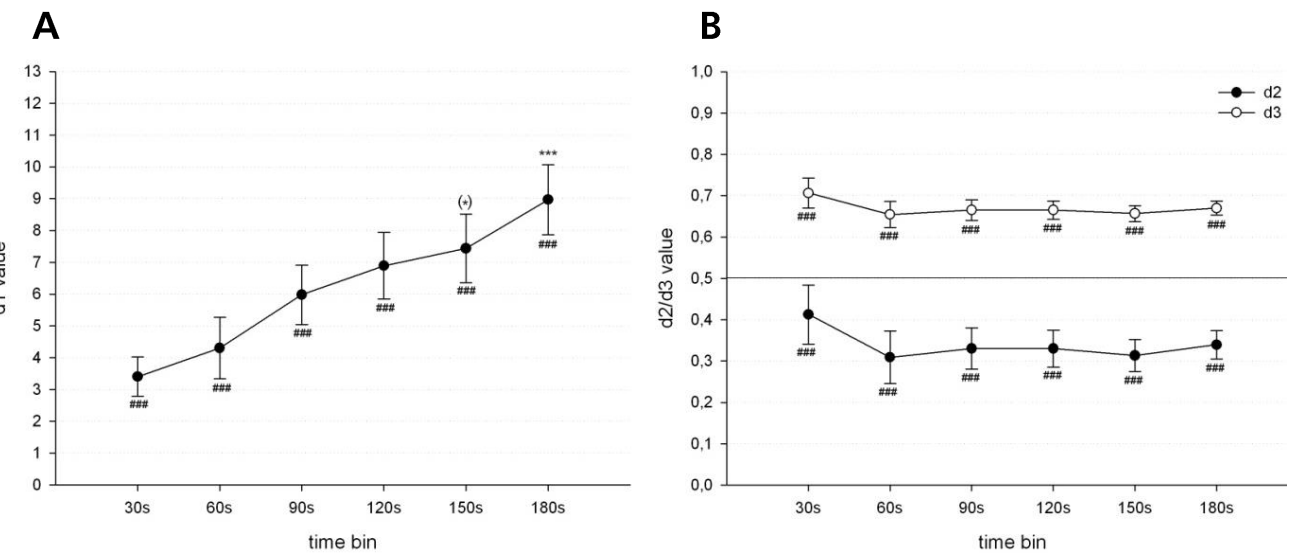

Figure 14 Distribution of discrimination within the test trial

Cumulative $d 1$ value $(\mathbf{A})$, or $d 2 / d 3$ value (B) given the hypothetical situation that the test trial $T 2$ would have been stopped after the time bin depicted on the x-axis. Differences from zero ( $d 1$ and $d 2)$ or $0.5(d 3)$ are indicated with hashes (one-sample $t$-tests, \#\#\# $<<0.001$ ). A significant difference from the first time bin is indicated with asterisks (Bonferroni $t$-tests, $\left.{ }^{*}\right) p<0.06 ;{ }^{*} p<0.05 ;{ }^{* *} p<0.001$ ). 


\section{Discussion}

\section{EXPLORATION AND DISCRIMINATION}

We found a large positive correlation between e1 and e2, indicating that there is consistency in the amount of exploration of individual animals. Correlation coefficients found between $\mathrm{e} 1$ and the $\mathrm{d}$ measures were slightly negative, indicating that higher exploration levels in the sample trial T1 are not associated with better discrimination performance, a phenomenon previously reported by Gaskin et al. (2010). This may suggest that the amount of exploration in $\mathrm{T} 1$ does not reflect the quality of memory encoding. On the other hand, it is also possible that discrimination ratios are not a valid estimate of memory strength, as was argued by Gaskin et al. (2010). The slightly negative correlation that was observed in our study might be explained by the assumption that rats with higher e1 values spent less time familiarizing with the environment (contextual information), which has been shown to increase novelty preference (Besheer \& Bevins, 2000; Wilkinson, et al., 2006). During each trial, animals distribute their attention over the objects and the arena. Therefore, a higher e1 measure might imply that animals spent less time exploring the arena and therefore acquired relatively less contextual information. If the context is less familiar in the test trial, the contrast between the novel object and the context might be a fraction lower. However, it should be noted that the low correlations only turned out significant because of the high number of subjects in our analysis, that is, the explained proportion of the total variance is very low.

In the test trial $\mathrm{T} 2$, exploration (e2) and $d 1$ had a high positive correlation, whereas e2 only had low positive correlations with $d 2$ and $d 3$. Therefore, $d 2$ and $d 3$ are less biased by exploratory activity. The latter was also observed in an object location test (OLT) using a protocol similar to ours, but, in T2, the location of one of the familiar objects is changed instead of replacing it by a novel object (Ozawa, Yamada, \& Ichitani, 2011). These findings make the $d 2$ and $d 3$ measures more suited for conditions that may affect the exploratory activity in animals, e.g., when testing 
pharmacological compounds or the effects of stress. Of note, again the low correlation had turned significant due to the high number of subjects.

Non-familiarized animals required at least $10 \mathrm{~s}$ of object interaction in T1 and $12 \mathrm{~s}$ in T2 for reliable object discrimination. This amount was lower for familiarized animals which needed $7 \mathrm{~s}$ and $10 \mathrm{~s}$ of object exploration in $\mathrm{T} 1 \mathrm{and} \mathrm{T} 2$, respectively. The majority of the animals reached this amount of exploration. In $T 1$, but $3.7 \%$ of the non-familiarized animals and $0.4 \%$ of the familiarized animals did not show sufficient exploration. The same pattern was found in $\mathrm{T} 2$, with insufficient exploration in $4.2 \%$ and $1.5 \%$ of nonfamiliarized and familiarized animals, respectively. This indicates that, when using non-familiarized animals, a substantial drop-out due to insufficient exploration levels has to be considered.

Within our 3 min trials, exploration kept increasing significantly over the first $90 \mathrm{~s}$. Both in $\mathrm{T} 1$ and $\mathrm{T} 2$, the first $90 \mathrm{~s}$ contributed roughly $66 \%$ of the total exploration, this corresponds to the findings of Dix and Aggleton (1999), who found that most discriminative exploration was displayed in the first 2 min of a trial. In our experiments $d 1$ and $d 2$ measures were already significantly higher than zero after a trial duration of $30 \mathrm{~s}$ in T2. The same holds for $d 3$ with respect to its reference value of 0.5 . Within this $30 \mathrm{~s}$ trial duration, the level of exploration in T2 was already $8 \mathrm{~s}$, which was approximately $30 \%$ of the total exploration and close to the required minimum amount of exploration (9-10 s) that we found to be necessary for reliable discrimination performance in familiarized animals. After a trial duration of $60 \mathrm{~s}$, the minimum exploration criterion is always met in T2. Indicating that, using our protocol, a trial duration of $60 \mathrm{~s}$ is already sufficient in $\mathrm{T} 2$. In a recent study using a similar test protocol, but for object location memory, Ozawa et al. (2011) observed the same minimum trial durations.

In contrast to Dix and Aggleton (1999), we found that the $d 1$ measure kept increasing with trial duration of $\mathrm{T} 2$, meaning that longer trials will intrinsically produce better discrimination performance. On the other hand, after a $1 \mathrm{~h}$ delay, the $d 2$ and $d 3$ measures were insensitive to the trial duration of $\mathrm{T} 2$ and remained stable during the whole trial duration of $3 \mathrm{~min}$. In a recent OLT study using a similar test protocol, it has 
been shown that at longer delays, when the memory trace is 'weaker', the $d 2$ measure will decrease after 1-2 min with increasing trial duration of T2 (Ozawa, et al., 2011). Nevertheless, the above observations make $d 2$ and $d 3$ the preferred measures for inter-experimental comparisons. However, when comparing experimental designs with a fixed exploration level, $d 1$ may also be a useful measure.

\section{FAMILIARIZATION}

In the $1 \mathrm{~h}$ familiarization protocol, animals discriminated significantly between the objects in T2 in all familiarization sessions. This finding indicates that naïve animals are intrinsically capable of object discrimination, so the ORT indeed appears to utilize the rat's natural tendency towards novelty (Ennaceur \& Delacour, 1988). The first two familiarization sessions were identical in both the $1 \mathrm{~h}$ familiarization protocol and $24 \mathrm{~h}$ familiarization protocol. It was found that e1 decreased from the 1 st to the 2 nd familiarization session, whereas the level of e2 remained the same. In addition, we observed that e1 was higher than e2 in the first familiarization session. It has been shown that exposure ( $3 \mathrm{~min}$ ) to a novel environment is sufficient to elicit a stress response in rats (Brown \& Martin, 1974; de Boer, Koopmans, Slangen, \& Van Der Gugten, 1990). The decrease in e1 from the 1st to the 2 nd familiarization session, as well as the decrease from e1 to e2 in the 1st familiarization session could be explained by behavioral habituation (Cerbone \& Sadile, 1994), since the stress response has been shown to decrease with repeated exposures to a stressor (de Boer, et al., 1990). On the other hand, the 1st familiarization session is the first time the animals encounter objects in the arena. Therefore, $e 1$ in this session may also be elevated out of curiosity or arousal.

In the $1 \mathrm{~h}$ familiarization protocol and the $24 \mathrm{~h}$ familiarization protocol, there was an increase in e2 in the 3rd session whereas e1 did not change from the 2 nd to the $3 r d$ session. In both protocols, the e2 value of the 3rd session was higher than the e2 value in any of the other sessions. The '3rd' familiarization session was the first session in which the animals were tested in combination with (saline) injections. This is likely to induce a stress reaction due to the discomfort of the injection procedures (Balcombe, Barnard, \& Sandusky, 2004; Sharp, Zammit, Azar, \& Lawson, 2003). In the 
our studies, animals received (minimally) one saline injection 30 min before T1 in the 1 $\mathrm{h}$ retention studies and in the $24 \mathrm{~h}$ retention studies animals received a vehicle injection between 0 min and $3 \mathrm{~h}$ after T1. It is, however, unlikely that e2 was directly affected by stress, since in the 3rd session e1 remained unchanged compared to the prior session, in which animals did not receive injections. Furthermore, in the $24 \mathrm{~h}$ familiarization protocol T2 was performed at least $21 \mathrm{~h}$ after the time of injection. After this interval the stress response will be normalized (de Boer, et al., 1990).

The increased exploration in T2 might the result of a memory effect. That is, the detection of a novel object may increase arousal and thereby exploratory activity. Indeed, all discrimination measures of the 3rd familiarization session were higher compared to any of other individual sessions except the 4th. Many studies have shown that stress can have varying effects on memory functioning (McGaugh, Roozendaal, \& John, 2008; McIntyre \& Roozendaal, 2007; McReynolds, et al., 2010; Okuda, Roozendaal, \& McGaugh, 2004; Roozendaal, Castello, Vedana, Barsegyan, \& McGaugh, 2008; Roozendaal, Hahn, Nathan, de Quervain, \& McGaugh, 2004). Memory can be facilitated when stress is experienced in the same context and around the time of learning (Joëls, Pu, Wiegert, Oitzl, \& Krugers, 2006). Therefore, experiencing the injections close to $\mathrm{T} 1$ for the first time might have enhanced memory formation during $\mathrm{T} 1$.

From the 3rd session onward, e1 measures did not significantly decrease anymore in the $1 \mathrm{~h}$ protocol and e2 values of the 1st, $2 \mathrm{nd}$ and 4 th sessions were all equal to that of the 5th session. In the $24 \mathrm{~h}$ training protocol, we observed an exploratory drop in the 4 th familiarization session. In this session, both e1 and e2 were significantly lower than in any of the other sessions. In the 5th test session of the $24 \mathrm{~h}$ training protocol, e1 returned to the level of the 3rd session. Also the level of e2 increased to a level identical to that of $e 1$, indicating that when the familiar object is not remembered the objects in $\mathrm{T} 2$ were equally interesting to the animals as those in T1. The drop in exploration in the 4 th session of the $24 \mathrm{~h}$ training protocol is difficult to explain, since animals underwent exactly the same procedure as in the 5th session. However, this 
finding might be incidental, since this condition only incorporated animals from 2 small experiments.

\section{NOVELTY}

In the $24 \mathrm{~h}$ familiarization protocol, all discrimination measures of the first 3 sessions were significantly above chance level, indicating that the animals discriminated between the objects. In the 1st session this is to be expected, since a $1 \mathrm{~h}$ retention interval was used. In the 2nd and 3rd session discrimination measures were significantly above chance level, indicating that the animals remembered the familiar object over a $24 \mathrm{~h}$ retention delay. However, from the 4 th session on the rats stopped discriminating between the novel and familiar object after a $24 \mathrm{~h}$ delay. This observation might be explained by the manner in which the 4 sets of objects were presented to the animals, as was therefore investigated into detail.

In our experiments, the object sets were rotated over the individual animals in such a way that, after the 3rd session, the novel object in 12 was the same as the familiar object from 3 sessions before. This might imply that Wistar rats are able to remember object information much longer than $24 \mathrm{~h}$, since in every experiment at least 9 days were interposed between the $1 \mathrm{st}$ and 4 th test sessions. It is even possible that rats permanently retain object information after a single test trial and after the first acquaintance only temporal information is updated. We found support for this view in the supplementary experiment in which we used two completely new sets of objects in a $24 \mathrm{~h}$ retention interval. In the first of 2 consecutive sessions, the fully familiarized animals again discriminated between the objects. In the following session, which was identical to the first one, discrimination had disappeared again. This supports the notion stated by Ennaceur (Ennaceur, 2010) that after the first single encounter with an object, rats only reconsolidate or perform a 'familiarity update' in successive encounters. We also found a marked decrease in overall exploration from the 1st to the 2 nd test session, which indicates a habituation effect that cannot be explained by environmental factors, since these remained the same. Animals only stopped discriminating between objects after a $24 \mathrm{~h}$ retention interval if they had had an encounter with the novel object in a previous session. Hence, natural 'forgetting' in a 
$24 \mathrm{~h}$ interval might not reflect the forgetting of the total object representation, but only that of the temporal aspect of the object representation. After the initial encounter with an object, animals probably reconsolidate the object information each time that an object is encountered, adding new contextual information (object location, last time encountered) to the already existing object representation. Therefore the object that was more recently encountered (i.e. the familiar object) is likely to be more familiar compared to a relatively less familiar object (i.e. the novel object), that is an object which's representation was less recently updated. This relative difference in familiarity is forgotten within $24 \mathrm{~h}$.

\section{PhARMACOLOGICAL DEFICIT MODELS}

In the experimental phase of our experiments, untreated and saline treated animals were well capable of discriminating between the novel and the old object and no differences were found between the $d$ measures of both conditions. As mentioned above, data from the familiarization protocols showed that animals that discriminate between objects have an increased exploration level in T2, as compared to T1. This was also the case in fully familiarized untreated and saline treated animals.

In the 5th session of the $24 \mathrm{~h}$ familiarization protocol, animals showed no discrimination above chance level and e2 remained equal to e1. This exploration pattern was also observed in scopolamine treated animals, which also did not discriminate between objects in T2. Scopolamine is a muscarinic receptor antagonist which, amongst others, is used to mimic cognitive deficits in Alzheimer's disease (Klinkenberg \& Blokland, 2010). The increase in e2 was markedly reduced compared to the $e 2$ of saline treated rats in the $1 \mathrm{~h}$ retention interval. However, the difference still turned out significant, due to the large dataset $(n=715)$.

MK-801 treated animals were also unable to discriminate between objects in T2, yet e2 was significantly increased compared to $e 1$, which was similar to the e1 of saline treated animals. MK-801 is a non-competitive NMDA receptor antagonist that is used as a model for schizophrenia because it mimics both positive and negative symptoms of the disorder (Rung, Carlsson, Rydén Markinhuhta, \& Carlsson, 2005). MK-801 is a 
potent cognition impairer, but sensory, locomotor and toxicological side effects have been shown to become problematic with doses higher than $1 \mathrm{mg} / \mathrm{kg}$ and may already influence ORT performance at lower doses (Eyjolfsson, Brenner, Kondziella, \& Sonnewald, 2006; van der Staay, et al., 2011). Young Wistar rats have been shown susceptible for non-cognitive side effects like increased locomotion and stereotypic movements at a dose of $0.5 \mathrm{mg} / \mathrm{kg}$. With age, animals become more resistant to these side effects (Pesic, et al., 2010). MK-801 reaches maximum brain concentrations, 30 min after (IP) administration (Vezzani, et al., 1989), thereby exactly coinciding with our T1. Increased locomotion has been reported to last up to $90 \mathrm{~min}$ after injection, whereas other side effects like ataxia and head weaving have shorter durations, of around $30 \mathrm{~min}$ and $60 \mathrm{~min}$, respectively (Pesic, et al., 2010; Vasilev, Veskov, Janac, Rakic, \& Stojiljkovic, 2003; Vezzani, et al., 1989). It might therefore be that, in T1, increased locomotion was masked by other side effects that are related to distorted perception and lack of coordination. In T2, on the other hand, these other side effects will have faded. Therefore, the increase in e2 might be explained by locomotor effects of MK-801 treatment.

\section{CONSIDERATIONS WHEN USING THE OBJECT RECOGNITION TASK}

The ORT is a sensitive, reliable memory assessment tool that is able to detect subtle behavioral and cognitive effects. However, although the basic drive behind the task is very straightforward, i.e. rats are drawn to novel objects, there are more underlying factors influencing behavior and memory performance. For example, injection stress increased exploration when animals experienced it for the first time and thus, may directly affect discrimination measures. It is therefore highly recommended that animals are familiarized to the complete testing and administration procedure during familiarization, to prevent bias during the experiment.

We found that, in our protocol, familiarized Wistar rats only require $7 \mathrm{~s}$ of exploration in $\mathrm{T} 1$ and $10 \mathrm{~s}$ of exploration in $\mathrm{T} 2$, to achieve reliable discrimination performance. Both in $\mathrm{T} 1$ and $\mathrm{T} 2$, animals already achieved these exploration levels after a trial duration of $60 \mathrm{~s}$. Additionally, only a weak negative relationship was found between $\mathrm{e} 1$ and the $\mathrm{d}$ measures. Therefore, it would be advisable to use fixed trial durations, 
since testing with an exploration threshold will lead to different trial durations for each animal which in turn will cause differences in context familiarization between animals. This familiarization bias (Besheer \& Bevins, 2000; Wilkinson, et al., 2006) can be minimized by using a fixed trial duration that is long enough for the animals to reach reliable discrimination performance. Using our testing conditions, both T1 and T2 should last at least $60 \mathrm{~s}$.

It is commonly believed that Wistar rats are unable to remember objects over a $24 \mathrm{~h}$ retention period, our findings, however, suggest that part of the object information is retained for a longer period as Ennaceur suspected (2010). Animals only stopped discriminating between objects after a $24 \mathrm{~h}$ retention interval if they had previously encountered the novel object. Taking these findings into account, one should familiarize the animals to all the different objects during familiarization or provide completely novel sets of objects for each test session to prevent confusing consolidation with reconsolidation processes.

Several different discrimination measures are used to report findings in the ORT and different names are used to indicate them. Basically, two types of discrimination measures can be used, absolute and relative measures. We found that the absolute discrimination measure $d 1$ is positively correlated to exploratory activity. Hence, more active/exploratory animals are assumed to show better discrimination compared to animals that are less exploratory active. This makes absolute discrimination measures vulnerable to any factors affecting exploration, like (injection) stress and drug-induced side effects. Relative discrimination measures such as $d 2$ and $d 3$ correct for a potential bias in exploratory activity and thus might be more suited for comparing ORT studies between laboratories. 


\section{REFERENCES}

Ainge, J. A., Heron-Maxwell, C., Theofilas, P., Wright, P., de Hoz, L., \& Wood, E. R. (2006). The role of the hippocampus in object recognition in rats: examination of the influence of task parameters and lesion size. Behav Brain Res, 167(1), 183-195.

Albasser, M. M., Davies, M., Futter, J. E., \& Aggleton, J. P. (2009). Magnitude of the Object Recognition Deficit Associated With Perirhinal Cortex Damage in Rats: Effects of Varying the Lesion Extent and the Duration of the Sample Period. Behavioral Neuroscience, 123(1), 115-124.

Andrews, J. S. (1996). Possible confounding influence of strain, age and gender on cognitive performance in rats. Cognitive Brain Research, 3(3-4), 251-267.

Andrews, J. S., Jansen, J. H. M., Linders, S., Princen, A., \& Broekkamp, C. L. E. (1995). Performance of four different rat strains in the autoshaping, two-object discrimination, and swim maze tests of learning and memory. Physiology \& Behavior, 57(4), 785-790.

Balcombe, J. P., Barnard, N. D., \& Sandusky, C. (2004). Laboratory routines cause animal stress. Contemp Top Lab Anim Sci, 43(6), 42-51.

Besheer, J., \& Bevins, R. A. (2000). The role of environmental familiarization in novelobject preference. Behav Processes, 50(1), 19-29.

Bevins, R. A., \& Besheer, J. (2006). Object recognition in rats and mice: a one-trial non-matching-to-sample learning task to study 'recognition memory'. Nat Protoc, 1(3), 1306-1311.

Brown, G. M., \& Martin, J. B. (1974). Corticosterone, Prolactin, and Growth Hormone Responses to Handling and New Environment in the Rat. Psychosomatic Medicine, 36(3), 241-247.

Callahan, H., Ikeda-douglas, C., Head, E., Cotman, C. W., \& Milgram, N. W. (2000). Development of a protocol for studying object recognition memory in the dog. Progress in Neuro-Psychopharmacology and Biological Psychiatry, 24(5), 693-707.

Cerbone, A., \& Sadile, A. G. (1994). Behavioral habituation to spatial novelty: interference and noninterference studies. Neurosci Biobehav Rev, 18(4), 497518.

Cohen, J. (1988). Statistical power analysis for the behavioral sciences (2nd ed.). Hillsdale, New Jersey: Lawrence Erlbaum Associates, Inc., Publishers.

de Boer, S. F., Koopmans, S. J., Slangen, J. L., \& Van Der Gugten, J. (1990). Plasma catecholamine, corticosterone and glucose responses to repeated stress in rats: Effect of interstressor interval length. Physiology \& Behavior, 47(6), 1117-1124. 
de Lima, M. N. M., Laranja, D. C., Bromberg, E., Roesler, R., \& Schröder, N. (2005). Pre- or post-training administration of the NMDA receptor blocker MK-801 impairs object recognition memory in rats. Behav Brain Res, 156(1), 139-143.

de Lima, M. N. M., Luft, T., Roesler, R., \& Schröder, N. (2006). Temporary inactivation reveals an essential role of the dorsal hippocampus in consolidation of object recognition memory. Neuroscience Letters, 405(1-2), 142-146.

Dere, E., Huston, J. P., \& De Souza Silva, M. A. (2007). The pharmacology, neuroanatomy and neurogenetics of one-trial object recognition in rodents. Neuroscience \& Biobehavioral Reviews, 31(5), 673-704.

Dickerson, B. C., \& Eichenbaum, H. (2010). The episodic memory system: neurocircuitry and disorders. Neuropsychopharmacology, 35(1), 86-104.

Dix, S. L., \& Aggleton, J. P. (1999). Extending the spontaneous preference test of recognition: evidence of object-location and object-context recognition. Behav Brain Res, 99(2), 191-200.

Ennaceur, A. (2010). One-trial object recognition in rats and mice: Methodological and theoretical issues. Behavioural Brain Research, 215(2), 244-254.

Ennaceur, A., \& Delacour, J. (1988). A new one-trial test for neurobiological studies of memory in rats. 1: Behavioral data. Behav Brain Res, 31(1), 47-59.

Ennaceur, A., Michalikova, S., Bradford, A., \& Ahmed, S. (2005). Detailed analysis of the behavior of Lister and Wistar rats in anxiety, object recognition and object location tasks. Behavioural Brain Research, 159(2), 247-266.

Eyjolfsson, E. M., Brenner, E., Kondziella, D., \& Sonnewald, U. (2006). Repeated injection of MK801: An animal model of schizophrenia? Neurochemistry International, 48(6-7), 541-546.

Frick, K. M., \& Gresack, J. E. (2003). Sex differences in the behavioral response to spatial and object novelty in adult C57BL/6 mice. Behav Neurosci, 117(6), 1283-1291.

Gaskin, S., Tardif, M., Cole, E., Piterkin, P., Kayello, L., \& Mumby, D. G. (2010). Object familiarization and novel-object preference in rats. Behav Processes, 83(1), 61-71.

Gifford, A. K., Cloutier, S., \& Newberry, R. C. (2007). Objects as enrichment: Effects of object exposure time and delay interval on object recognition memory of the domestic pig. Applied Animal Behaviour Science, 107(3-4), 206-217.

Hanggi, E. B. (2010). Rotated object recognition in four domestic horses (equus caballus). Journal of Equine Veterinary Science, 30(4).

Hughes, R. N. (2007). Neotic preferences in laboratory rodents: Issues, assessment and substrates. Neuroscience \& Biobehavioral Reviews, 31(3), 441-464.

Joëls, M., Pu, Z., Wiegert, O., Oitzl, M. S., \& Krugers, H. J. (2006). Learning under stress: how does it work? Trends in Cognitive Sciences, 10(4), 152-158. 
Klinkenberg, I., \& Blokland, A. (2010). The validity of scopolamine as a pharmacological model for cognitive impairment: a review of animal behavioral studies. Neurosci Biobehav Rev, 34(8), 1307-1350.

Kornum, B. R., Thygesen, K. S., Nielsen, T. R., Knudsen, G. M., \& Lind, N. M. (2007). The effect of the inter-phase delay interval in the spontaneous object recognition test for pigs. Behavioural Brain Research, 181(2), 210-217.

McGaugh, J. L., Roozendaal, B., \& John, H. B. (2008). Memory Modulation Learning and Memory: A Comprehensive Reference (pp. 521-553). Oxford: Academic Press.

McIntyre, C. K., \& Roozendaal, B. (2007). Adrenal Stress Hormones and Enhanced Memory for Emotionally Arousing Experiences.

McReynolds, J. R., Donowho, K., Abdi, A., McGaugh, J. L., Roozendaal, B., \& Mclntyre, C. K. (2010). Memory-enhancing corticosterone treatment increases amygdala norepinephrine and Arc protein expression in hippocampal synaptic fractions. Neurobiol Learn Mem, 93(3), 312-321.

Mumby, D. G. (2001). Perspectives on object-recognition memory following hippocampal damage: lessons from studies in rats. Behavioural Brain Research, 127(1-2), 159-181.

Mumby, D. G., Gaskin, S., Glenn, M. J., Schramek, T. E., \& Lehmann, H. (2002). Hippocampal damage and exploratory preferences in rats: memory for objects, places, and contexts. Learn Mem, 9(2), 49-57.

Mumby, D. G., Piterkin, P., Lecluse, V., \& Lehmann, H. (2007). Perirhinal cortex damage and anterograde object-recognition in rats after long retention intervals. Behavioural Brain Research, 185(2), 82-87.

Norman, G., \& Eacott, M. J. (2004). Impaired object recognition with increasing levels of feature ambiguity in rats with perirhinal cortex lesions. Behavioural Brain Research, 148(1-2), 79-91.

O'Brien, N., Lehmann, H., Lecluse, V., \& Mumby, D. G. (2006). Enhanced contextdependency of object recognition in rats with hippocampal lesions. Behavioural Brain Research, 170(1), 156-162.

Okuda, S., Roozendaal, B., \& McGaugh, J. L. (2004). Glucocorticoid effects on object recognition memory require training-associated emotional arousal. Proc Natl Acad Sci U S A, 101(3), 853-858.

Ozawa, T., Yamada, K., \& Ichitani, Y. (2011). Long-term object location memory in rats: Effects of sample phase and delay length in spontaneous place recognition test. Neuroscience Letters, 497(1), 37-41.

Pesic, V., Popic, J., Milanovic, D., Loncarevic-Vasiljkovic, N., Rakic, L., Kanazir, S., et al. (2010). The effect of MK-801 on motor activity and c-Fos protein expression in the brain of adolescent Wistar rats. Brain Res, 1321, 96-104. 
Prickaerts, J., de Vente, J., Honig, W., Steinbusch, H. W., \& Blokland, A. (2002). CGMP, but not CAMP, in rat hippocampus is involved in early stages of object memory consolidation. Eur J Pharmacol, 436(1-2), 83-87.

Prickaerts, J., Sik, A., van der Staay, F. J., de Vente, J., \& Blokland, A. (2005). Dissociable effects of acetylcholinesterase inhibitors and phosphodiesterase type 5 inhibitors on object recognition memory: acquisition versus consolidation. Psychopharmacology (Berl), 177(4), 381-390.

Prickaerts, J., van Staveren, W. C. G., Sik, A., Markerink-van Ittersum, M., Niewöhner, U., van der Staay, F. J., et al. (2002). Effects of two selective phosphodiesterase type 5 inhibitors, sildenafil and vardenafil, on object recognition memory and hippocampal cyclic GMP levels in the rat. Neuroscience, 113(2), 351-361.

Roozendaal, B., Castello, N. A., Vedana, G., Barsegyan, A., \& McGaugh, J. L. (2008). Noradrenergic activation of the basolateral amygdala modulates consolidation of object recognition memory. Neurobiol Learn Mem, 90(3), 576-579.

Roozendaal, B., Hahn, E. L., Nathan, S. V., de Quervain, D. J., \& McGaugh, J. L. (2004). Glucocorticoid effects on memory retrieval require concurrent noradrenergic activity in the hippocampus and basolateral amygdala. J Neurosci, 24(37), 8161-8169.

Rung, J. P., Carlsson, A., Rydén Markinhuhta, K., \& Carlsson, M. L. (2005). (+)-MK801 induced social withdrawal in rats; a model for negative symptoms of schizophrenia. Progress in Neuro-Psychopharmacology and Biological Psychiatry, 29(5), 827-832.

Rutten, K., Prickaerts, J., Hendrix, M., van der Staay, F. J., Sik, A., \& Blokland, A. (2007). Time-dependent involvement of cAMP and cGMP in consolidation of object memory: studies using selective phosphodiesterase type 2, 4 and 5 inhibitors. Eur J Pharmacol, 558(1-3), 107-112.

Rutten, K., Prickaerts, J., Schaenzle, G., Rosenbrock, H., \& Blokland, A. (2008). Subchronic rolipram treatment leads to a persistent improvement in long-term object memory in rats. Neurobiol Learn Mem, 90(3), 569-575.

Rutten, K., Reneerkens, O. A. H., Hamers, H., Sik, A., McGregor, I. S., Prickaerts, J., et al. (2008). Automated scoring of novel object recognition in rats. Journal of Neuroscience Methods, 171(1), 72-77.

Rutten, K., Van Donkelaar, E. L., Ferrington, L., Blokland, A., Bollen, E., Steinbusch, H. W., et al. (2009). Phosphodiesterase inhibitors enhance object memory independent of cerebral blood flow and glucose utilization in rats. Neuropsychopharmacology, 34(8), 1914-1925.

Sambeth, A., Riedel, W. J., Smits, L. T., \& Blokland, A. (2007). Cholinergic drugs affect novel object recognition in rats: Relation with hippocampal EEG? European Journal of Pharmacology, 572(2-3), 151-159. 
Schenk, S., Harper, D. N., \& Do, J. (2010). Novel object recognition memory: measurement issues and effects of MDMA self-administration following short inter-trial intervals. J Psychopharmacol.

Sharp, J., Zammit, T., Azar, T., \& Lawson, D. (2003). Stress-like responses to common procedures in individually and group-housed female rats. Contemp Top Lab Anim Sci, 42(1), 9-18.

Sik, A., van Nieuwehuyzen, P., Prickaerts, J., \& Blokland, A. (2003). Performance of different mouse strains in an object recognition task. Behavioural Brain Research, 147, 49-54.

van der Staay, F. J., Rutten, K., Erb, C., \& Blokland, A. (2011). Effects of the cognition impairer MK-801 on learning and memory in mice and rats. Behav Brain Res, 220(1), 215-229.

van Goethem, N. P., Rutten, K., van der Staay, F. J., Jans, L. A. W., Akkerman, S., Steinbusch, H. W. M., et al. (2012). Object recognition testing: Rodent species, strains, housing conditions, and estrous cycle. Behavioural Brain Research, 232(2), 323-334.

Vasilev, V., Veskov, R., Janac, B., Rakic, L., \& Stojiljkovic, M. (2003). Age-related differences in MK-801- and amphetamine-induced locomotor and stereotypic activities of rats. Neurobiol Aging, 24(5), 715-723.

Vezzani, A., Serafini, R., Stasi, M. A., Caccia, S., Conti, I., Tridico, R. V., et al. (1989). Kinetics of MK-801 and its effect on quinolinic acid-induced seizures and neurotoxicity in rats. J Pharmacol Exp Ther, 249(1), 278-283.

Wilkinson, J. L., Herrman, L., Palmatier, M. I., \& Bevins, R. A. (2006). Rats' novel object interaction as a measure of environmental familiarity. Learning and Motivation, 37(2), 131-148.

Winters, B. D., \& Bussey, T. J. (2005). Transient Inactivation of Perirhinal Cortex Disrupts Encoding, Retrieval, and Consolidation of Object Recognition Memory. The Journal of Neuroscience, 25(1), 52-61.

Winters, B. D., Forwood, S. E., Cowell, R. A., Saksida, L. M., \& Bussey, T. J. (2004). Double Dissociation between the Effects of Peri-Postrhinal Cortex and Hippocampal Lesions on Tests of Object Recognition and Spatial Memory: Heterogeneity of Function within the Temporal Lobe. I Neurosci, 24(26), 5901-5908.

Winters, B. D., Saksida, L. M., \& Bussey, T. J. (2008). Object recognition memory: Neurobiological mechanisms of encoding, consolidation and retrieval. Neuroscience \& Biobehavioral Reviews, 32(5), 1055-1070. 

Chapter 3 


\section{OBJECT RECOGNITION TESTING: STATISTICAL CONSIDERATIONS}

Sven Akkerman, Jos Prickaerts, Harry W.M. Steinbusch and Arjan Blokland

Behavioural Brain Research (2012), 232, p. 317 - 322 


\section{ABSTRACT}

The object recognition task is a commonly used test for the assessment of memory functions in rodents. In this paper different aspects concerning the analysis of object recognition data are discussed. Using a set of experimental studies and fictive data sets, it was shown that the absolute discrimination measure $(d 1)$ behaves different from the ratio measures ( $d 2$ and $d 3$ ). Furthermore, it is suggested that, besides group differences, one should also examine whether each individual group actually discriminates between the novel and the familiar object. For this purpose, the use of a fictive group is advisable. On basis of 48 historical studies it is shown that discrimination performance does not fall significantly below zero. Therefore one-sided testing is allowed, provided that place- or object biases can be ruled out. Finally, it was shown that differences in exploration levels may affect the statistical evaluation of group differences. Several suggestions for statistical analysis are given. 


\section{INTRODUCTION}

The object recognition task (ORT) has proven to be a very useful task for the assessment of memory functions in the animals and has several characteristics that make it especially suitable for studying the neurobiology of memory (J.P. Aggleton, 1985; Ennaceur \& Delacour, 1988). Because it is based on a natural curiosity towards novelty, i.e. an inclination to explore novel objects, there is no need for any positive or negative reinforcement that could confound the data. Furthermore, the test is a onetrial learning task, which allows researchers to separately investigate different memory processes, like acquisition, consolidation and retrieval independently (Prickaerts, Sik, Van Der Staay, De Vente, \& Blokland, 2005; Rutten, Prickaerts, et al., 2007).

Since the first introduction of the test, different measures of discrimination have been proposed to analyze the novelty preference of the animals. Also the possible influence of the exploration levels on these discrimination parameters has been subject of discussion (Ennaceur, Michalikova, Bradford, \& Ahmed, 2005; Sik, van Nieuwehuyzen, Prickaerts, \& Blokland, 2003). Consequently, some researchers suggest that a minimal/fixed amount of exploration time is required (Kertesz, Kapus, Gacsalyi, \& Levay, 2010; Pichat, et al., 2007) in the first trial of the ORT whereas others use a fixed trial duration, regardless of the amount time animals spend exploring the objects (Ennaceur \& Delacour, 1988; Prickaerts, et al., 2005; Rutten, Lieben, Smits, \& Blokland, 2007; Snigdha \& Neill, 2008).

In this paper these aspects of the interpretation of ORT data were investigated. First, we discuss the different discrimination measures that are frequently used in the literature. At the moment the absolute difference in exploration (i.e. d1) (King, et al., 2004; Messier, 1997) and the relative measure (i.e. d2) (Gaskin, Tremblay, \& Mumby, 2003; Lieben, et al., 2005) of discrimination are most frequently reported. Secondly, different analyses are considered that can be relevant for the interpretation of the discrimination performance of animals in the ORT. Usually, a comparison of each individual experimental group with 'random exploration' should be performed in order to examine whether animals in these groups actually discriminate between the 
objects. In addition, discrimination performance is compared between different experimental groups of animals. Here, we suggest a method to analyze this discrimination performance. Thirdly, experimental treatments can have an effect on exploration, which might affect the comparison of discrimination performance. Therefore, the effects of low and high exploration levels on the different discrimination measures will be evaluated. Finally, it is demonstrated that under normal testing conditions the discrimination performance will not drop below a level of random exploration, i.e. animals will show no preference for familiar objects. This would suggest that one-tailed testing is allowed under these conditions.

To address these different ORT related issues, we used two different data sets. One data set consisted of data from previous ORT experiments performed in our lab. This data set contained ORT sessions in which rats either showed good discrimination performance ( $1 \mathrm{~h}$ inter-trial interval) or poor discrimination performance $(24 \mathrm{~h}$ intertrial interval, or scopolamine- and MK-801 induced memory deficits in a $1 \mathrm{~h}$ inter-trial delay). A second data set (consisting of four different fictive groups) was created in order to specifically test the characteristics of the different discrimination measures and whether they are affected by differences in exploration levels. This database was constructed since the real data sets did not contain these specific conditions.

\section{METHODS}

\section{ANIMALS}

In the present study we used data from a series of 18 ORT studies in which different drugs and inter-trial intervals (84 test sessions, with a total number of subjects: $n=$ 731) were tested. In all these studies male Wistar rats ( 3 to 5 months old; Harlan, The Netherlands) were used. They were individually housed in standard MakrolonTM Type III cages on sawdust bedding and had free access to food and water. The animals were kept under a reversed $12 \mathrm{~h}$ day/night cycle (lights were on between 19:00 and 07:00). Room temperature was kept constant at $20^{\circ} \mathrm{C}$. 


\section{Apparatus}

The rats were tested in a circular arena (diameter $83 \mathrm{~cm})$. In the first trial $(3 \mathrm{~min})$, two identical objects were symmetrically placed near the center of the arena. In the second trial ( $3 \mathrm{~min}$ ), one of the objects from the first trial was replaced by an exact copy (familiar object), the other object was replaced by a novel object. In total, 4 different sets of 3 identical objects were used. For a more detailed description of these task, objects, and procedures see (Akkerman, et al., 2012; Prickaerts, et al., 2005; Rutten, Prickaerts, et al., 2007).

\section{STATISTICAL ANALYSIS}

The basic measures of the ORT are the exploration times on the objects. On basis of these exploration times different discrimination measures can be calculated. In Table 1 , an overview of the most common discrimination measures and their calculations is given (for additional measures see: Akkerman, et al., 2012; Ennaceur, et al., 2005). First, $t$-tests are used to compare the discrimination measure(s) of each experimental condition with the discrimination outcome that would result from random exploration. This 'random value' will theoretically be zero in case of $d 1$ or $d 2$ and 0.5 when the $d 3$ measure is used. Comparison with random exploration will reveal whether or not animals in a condition discriminated between the objects in $\mathrm{T} 2 \mathrm{a}$ significant outcome indicates that exploration was not random and implies that the objects from T1 were remembered. Additionally, ANOVAs can be used to compare the level of discrimination between treatment conditions.

Table 1 Measures in the ORT

\begin{tabular}{cl}
\hline Exploration & Discrimination \\
\hline$e 1=a_{1}+a_{2}$ & $d 1=b-a_{3}$ \\
& $d 2=d 1 / e 2$ \\
$e 2=a_{3}+b$ & $d 3=b / e 2$ (or as \%; b/e $2 * 100)$ \\
\hline
\end{tabular}

Exploration and discrimination measures in object recognition testing. e1 refers to the total exploration in the first trial and e2 to the total exploration in the second trial. a1 and a2 refer to the exploration times on the two similar objects in the first trial. a3 refers to the exploration of the copy of objects a 1 and a2 in the second trial. $b$ refers to the time spent on the novel object in the second trial. 
Using one-sample $t$-tests, we compared 84 ORT sessions to zero in order to examine whether the $d 2$ measure is likely to drop below zero (random exploration). In addition to this historical data, we also created fictive data sets (groups with 19 subjects per group, see Table 2 and 5 for the characteristics) to compare the characteristics of the different discrimination parameters that are being used in the literature. The standard error of the mean (SEM) of these fictive groups was always corresponded to actual SEM values found in previous ORT studies using Wistar rats (Prickaerts, et al., 2005; Sik, et al., 2003). These fictive data sets were also used to examine discrimination when comparing with zero or with a fictive group that does not discriminate between objects. Comparisons were made using one-sample $t$-tests (see also Prickaerts, et al., 2005; Rutten, Prickaerts, Schaenzle, Rosenbrock, \& Blokland, 2008; Sik, et al., 2003). Finally, the same fictive data sets were also used to demonstrate the effects of low- vs, high exploration levels on the different discrimination parameters.

\section{RESULTS}

\section{DISCRIMINATION MEASURES}

To compare the characteristics of the different discrimination measures two fictive data sets (Group 1 and Group 2). Table 2 demonstrates that, although the calculations for $d 2$ and $d 3$ are different, both measures render exactly the same statistical outcome. The $d 1$ measure will return a different $p$-value compared to the other two discrimination measures because it does not correct for differences in exploration.

This example shows that the comparison of two groups that differ in discrimination performance reveals exactly the same statistical results, regardless of whether the $d 2$ or $d 3$ measure is used. Therefore, it seems that $d 2$ and $d 3$ measures can be used interchangeably. This is not the case for the uncorrected difference score $d 1$. The difference between the $d 1$ measure on the one hand and $d 2 / d 3$ measures on the other hand may appear small, with an absolute difference of 0.083 between $p$-values. However, this is a relevant difference because may lead to completely opposite 
Table 2 Comparison between discrimination measures

\begin{tabular}{cccccccccc}
\hline Measure & Group $(\boldsymbol{n}=\mathbf{1 9})$ & e2 (SEM) & a3 & $\boldsymbol{b}$ & Mean (SEM) & Mean difference & $\boldsymbol{t}$-value & $\mathbf{d f}$ & $\boldsymbol{p}$-value \\
\hline \multirow{2}{*}{$d 1$} & 1 & $33.80(1.51)$ & 14.40 & 19.40 & $5.00(2.31)$ & 4.842 & 1.33 & 36 & 0.191 \\
& 2 & $34.40(1.39)$ & 17.05 & 17.35 & $0.15(2.78)$ & & & & \\
\multirow{2}{*}{$d 2$} & 1 & $33.80(1.51)$ & 14.40 & 19.40 & $0.112(0.07)$ & 0.109 & 1.11 & 36 & 0.274 \\
& 2 & $34.40(1.39)$ & 17.05 & 17.35 & $0.003(0.07)$ & & & & \\
\multirow{2}{*}{$d 3$} & 1 & $33.80(1.51)$ & 14.40 & 19.40 & $0.556(0.03)$ & 0.055 & 1.11 & 36 & 0.274 \\
& 2 & $34.40(1.39)$ & 17.05 & 17.35 & $0.501(0.03)$ & & & & \\
\hline
\end{tabular}

Statistics, $t$-values and associated $p$-values of the three different discrimination parameters of the object recognition test. Analysis reflects the comparison of two FICT groups differing in discrimination performance. Exploration levels were comparable in both groups (Group 1: e2 $=33.8 \mathrm{~s}$ (SEM 1.51); Group $2: e 2=34.4$ s (SEM 1.39).

conclusions about whether or not an group of animals discriminates, as can be seen in the example portrayed in Table 3.

\section{COMPARISON WITH CONTROL GROUP AND ZERO}

When the effects of a certain treatment are investigated, an analysis is performed in which the control and experimental group are compared to each other. This analysis will reveal whether the treatment improved or impaired the object discrimination performance of the experimental rats. On the other hand, it is even more important to know whether the subjects actually do remember the familiar object. Consequently, it should first be tested whether the discrimination measures are actually different from zero (or in case of $d 3$ : different from 0.5). This is normally tested with a one sample $t$ test compared to 0 (or 0.5). However, this test may not be the optimal method of comparison, since it compares against a fixed value (0) without any changes in variance. Variance will always be present in a natural group of non-discriminating animals, and using a one-sample $t$-test will therefore increase the likelihood of making a Type I error. Ideally, the performance of a group should be compared to another group with a mean $d 1$ or $d 2$ of 0 (or 0.5 for $d 3$; no discrimination) and a standard deviation that is usually observed in the same type of experiments. To obtain an estimation of the variance of data we calculated the mean value of 10 random independent experiments. On basis of these data, a fictive (FICT) group was constructed. Although this can be considered to be an arbitrary choice, to our knowledge it will provide the most realistic picture about whether the performance of a specific experimental group differs from random exploration, i.e. if the experimental group truly shows a preference for the novel object. Hence, a FICT group was created 
with a calculated mean (SEM) for $d 1$ and $d 2$ of $0.15(2.79)$ and $0(0.07)$, respectively (for characteristics of FICT group, see Table 3).

Table 3 Analysis of discrimination performance

\begin{tabular}{|c|c|c|c|c|c|c|c|}
\hline $\begin{array}{l}\text { Discrimination } \\
\text { measure }\end{array}$ & $\begin{array}{l}\text { Group } \\
(n=19)\end{array}$ & Mean & SEM & Mean difference & t-value & df & $p$-value \\
\hline \multirow[t]{6}{*}{$d 1$} & EXP1 & 5.00 & 2.32 & & & & \\
\hline & ZERO & 0 & 0 & $5.0($ EXP1 - ZERO $)$ & 2.15 & 18 & $0.046^{*}$ \\
\hline & $\mathrm{FICT}$ & 0.15 & 2.78 & $4.8($ EXP1 - FICT) & 1.33 & 36 & 0.191 \\
\hline & EXP2 & 7.7 & 2.04 & & & & \\
\hline & ZERO & 0 & 0 & 7.7 (EXP2 - ZERO) & 3.43 & 18 & $0.003 * *$ \\
\hline & $\mathrm{FICT}$ & 0.15 & 2.78 & $7.5(\mathrm{EXP2}-\mathrm{FICT})$ & 2.10 & 36 & $0.043^{*}$ \\
\hline \multirow[t]{6}{*}{$d 2$} & EXP1 & 0.11 & 0.7 & & & & \\
\hline & ZERO & 0 & 0 & 0.11 (EXP1 - ZERO) & 1.61 & 18 & 0.126 \\
\hline & $\mathrm{FICT}$ & 0.00 & 0.07 & $0.11(\mathrm{EXP} 1-\mathrm{FICT})$ & 1.11 & 36 & 0.274 \\
\hline & EXP2 & 0.17 & 0.07 & & & & \\
\hline & ZERO & 0 & 0 & $0.17($ EXP2 - ZERO $)$ & 2.49 & 18 & $0.023^{*}$ \\
\hline & $\mathrm{FICT}$ & 0.00 & 0.07 & $0.17(\mathrm{EXP} 2-\mathrm{FICT})$ & 1.74 & 36 & 0.091 \\
\hline
\end{tabular}

Analysis of the performance of two fictive experimental groups (EXP1 and EXP2), differing in discrimination performance (EXP2 > EXP1), and the FICT group (discrimination performance zero). Exploration levels were comparable between all groups. For the group differences EXP1 vs. FICT and EXP2 vs. FICT the $d 1$ and $d 2$ the data were analyzed using an independent sample $t$-test. For the group difference EXP1 vs. ZERO and EXP2 vs. ZERO the $d 1$ and $d 2$ the data were analyzed using a one-sample t-test $\left({ }^{*}: p<0.05 ;{ }^{*}: p<0.01\right)$.

As can be seen in Table 3, the analyses of two fictive experimental (EXP) groups revealed statistically significant differences when comparing with the zero condition (one-sample $t$-test). Comparison with the FICT group was more conservative.

As mentioned earlier, comparison with zero or a FICT group allows the evaluation whether animals discriminate between the familiar and novel object (see Table 3). This analysis should always be performed prior to the comparison between a control and experimental group, in order to examine whether or not the animals do discriminate. By a control group we mean an actual experimental condition in which animals are 'sham' treated in order to determine baseline performance under the specific local testing conditions. When using these two separate within- and between subjects analyses, three possible conclusions can be hypothesized, a full effect, intermediate effect, or no effect (see Table 4). 
Table 4 Potential ORT outcomes

\begin{tabular}{|c|c|c|}
\hline $\begin{array}{l}\text { Comparison } \\
\text { experimental and } \\
\text { control group }\end{array}$ & $\begin{array}{l}\text { Comparison } \\
\text { experimental group } \\
\text { and zero (FICT group) }\end{array}$ & Conclusion \\
\hline \multicolumn{3}{|l|}{ A } \\
\hline No difference & No difference & $\begin{array}{l}\text { No effect of the experimental condition but animals also } \\
\text { do not discriminate between the objects. Indication of an } \\
\text { intermediate effect. }\end{array}$ \\
\hline No difference & Difference & $\begin{array}{l}\text { No effect of experimental condition, animals discriminate } \\
\text { between objects. No effect. }\end{array}$ \\
\hline Difference & No difference & $\begin{array}{l}\text { Experimental condition impairs discrimination } \\
\text { performance, animals do not discriminate between } \\
\text { objects. Full effect. }\end{array}$ \\
\hline Difference & Difference & $\begin{array}{l}\text { The experimental condition impairs discrimination } \\
\text { performance but the animals still discriminate between } \\
\text { objects. Indication of an intermediate effect. }\end{array}$ \\
\hline \multicolumn{3}{|l|}{ B } \\
\hline No difference & No difference & $\begin{array}{l}\text { No effect of experimental condition, animals do not } \\
\text { discriminate between objects. No effect. }\end{array}$ \\
\hline No difference & Difference & $\begin{array}{l}\text { No effect of experimental condition, animals discriminate } \\
\text { between objects. Intermediate effect }\end{array}$ \\
\hline Difference & No difference & $\begin{array}{l}\text { Experimental condition has no effect on discrimination } \\
\text { performance, animals do not discriminate between } \\
\text { objects. }\end{array}$ \\
\hline Difference & Difference & $\begin{array}{l}\text { The experimental condition improves discrimination } \\
\text { performance, animals discriminate between objects. Full } \\
\text { effect. }\end{array}$ \\
\hline
\end{tabular}

Possible conclusions about the effectiveness of a treatment when comparing an experimental group with both zero (FICT group) and a control (sham treated) group. (A) The control group does discriminate between the objects, e.g. when comparing with an experimentally induced memory deficit and (B) the control group does not discriminate between the objects, e.g. when comparing with an experimentally induced memory improvement. ${ }^{2}$ Likely related to a relatively great variance in the data or poor performance of control group. ${ }^{b}$ Likely related to a relatively small variance in the data or good performance of control group. ' Will only be observed in case the control condition is slightly negative or positive and variance within groups is very small.

\section{EXPLORATION LEVELS AND DISCRIMINATION PERFORMANCE}

In order to examine the consequences of differences in exploration levels on the statistical outcome of the discrimination measures, we prepared a fictive data set with four groups with high or low exploration levels in T2, combined with either good discrimination performance or no discrimination (see Table 5). Although these were fictive data sets, the $d 2$ values, the exploration levels and associated variance are highly comparable to experimental data obtained in our original studies. 
Table 5 Fictive groups

\begin{tabular}{llllll}
\hline Group & Exploration & Discrimination & e2 (s) & $\boldsymbol{d 1}$ & $\boldsymbol{d} \mathbf{2}$ \\
\hline 1 & Low & Low & $9.4(0.54)$ & $0.05(0.62)$ & $0.00(0.07)$ \\
2 & Low & High & $9.4(0.50)$ & $2.26(0.66)^{*}$ & $0.23(0.07)^{\star}$ \\
3 & High & Low & $31.4(1.47)$ & $1.00(2.57)$ & $-0.00(0.07)$ \\
4 & High & High & $31.2(1.91)$ & $8.37(2.04)^{*}$ & $0.24(0.07)^{*}$ \\
\hline
\end{tabular}

Description of four different fictive groups differing in exploration level and discrimination performance. Entries represent mean (SEM). Asterisks indicate a difference from zero (one-sample $t$-tests, $*: p<0.05$ ).

Using the data of these four groups we analyzed the group differences in a pair-wise manner. Thus, we examined whether the good or no discrimination performance for the low exploration conditions differed using an independent sample $t$-test. This was also done for the high exploration levels. In addition, we also examined the outcome of the independent sample $t$-test when comparing groups with different exploration levels. In Table 6 the outcome of the different comparisons is presented.

As can be seen in Table 6 , the absence of the effect on the $d 1$ measure in the comparison of groups 3 and 2 is related to the fact that a relatively low $d 1$ value can already be different from zero in case of low exploration levels, due to a low SEM (see Table 5). As a consequence, this low exploration $d 1$ performance $(2.26(0.66))$ is not different from a low $d 1$ value $(1.00(2.57))$ in case of high exploratory activity. Since the $d 2$ value corrects for differences in the absolute exploration levels, this observation was not found for this measure. This example shows that the sensitivity of the $d 1$ measure is sensitive to changes in exploration and SEM.

Table 6 Exploration level and discrimination statistics

\begin{tabular}{llll}
\hline Comparison & \multicolumn{1}{c}{$\boldsymbol{d 1}$} & $\boldsymbol{d} \mathbf{2}$ \\
\cline { 1 - 2 } Expl. - Discr. & Expl. - Discr. & & \\
\hline Low - Low (1) & Low - High (2) & $t_{(36)}=2.43, p<0.020$ & $t_{(36)}=2.36, p<0.024$ \\
High - Low (3) & Low - High (2) & $t_{(36)}=0.48$, n.S. & $t_{(36)}=2.33, p<0.026$ \\
Low - Low (1) & High - High (4) & $t_{(36)}=-3.90, p<0.001$ & $t_{(36)}=-2.58, p<0.01$ \\
High - Low (3) & High - High (4) & $t_{(36)}=2.25, p<0.031$ & $t_{(36)}=2.54, p<0.015$ \\
\hline
\end{tabular}

Statistical analysis between groups with low or high exploration levels and good or poor discrimination performance. The italic numbers between parentheses in the comparison columns refer the group numbers in Table 5. Note that only comparisons were made between groups differing in the discrimination performance. 


\section{VARIATIONS IN D2 VALUES}

In Fig. 1A an overview is given from a selection of 48 test sessions in which we used conditions which have shown to impair discrimination performance in previous experiments, namely; the NMDA antagonist MK-801 (0.125 mg/kg IP), the muscarinic antagonist scopolamine $(0.1 \mathrm{mg} / \mathrm{kg} \mathrm{IP})$, and a $24 \mathrm{~h}$ delay interval.
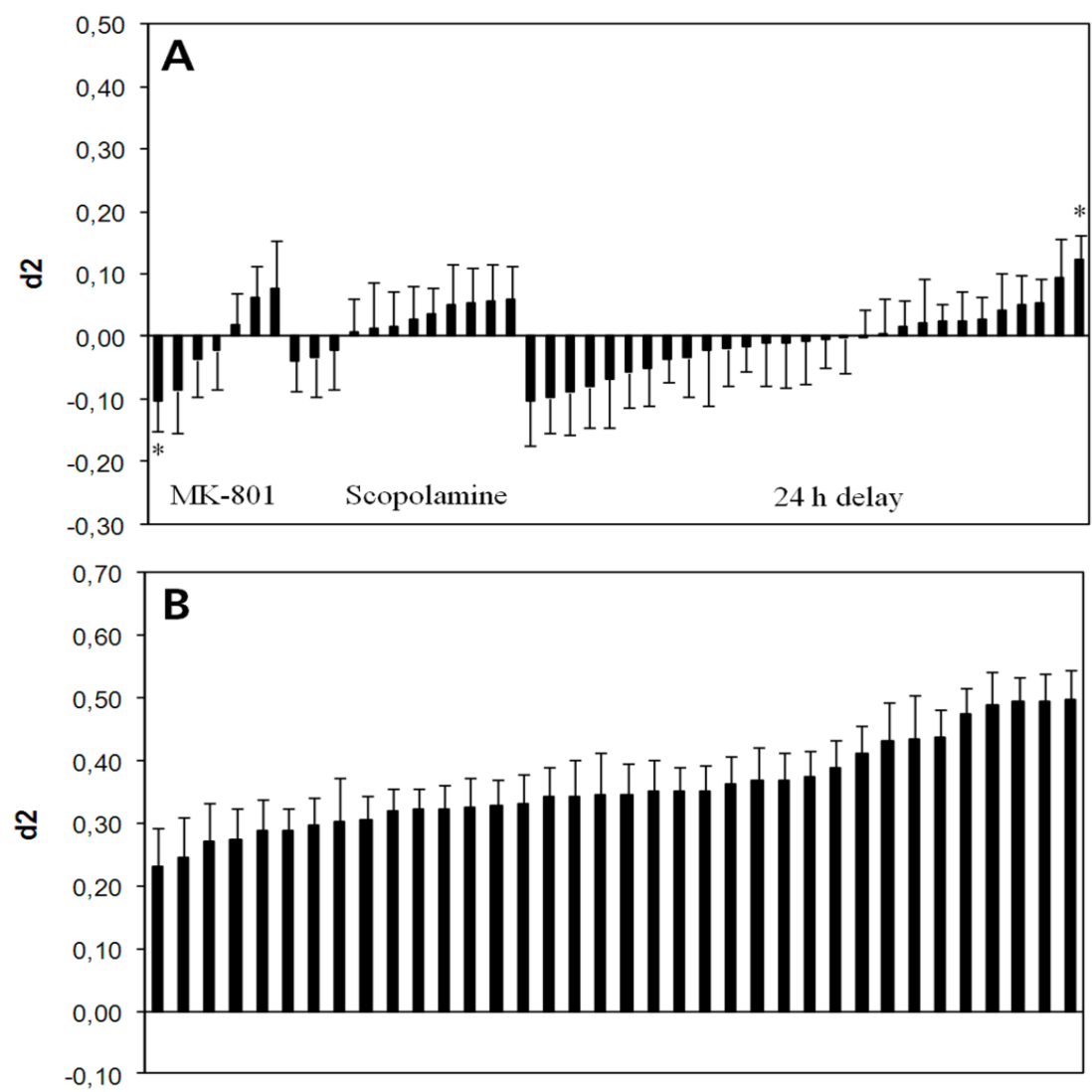

Figure 1 Historical ORT sessions

Discrimination performance (mean $d 2+$ SEM) of rats in the ORT (A) under conditions of MK-801 (1 h delay), or scopolamine induced forgetting ( $1 \mathrm{~h}$ delay) and natural forgetting ( $24 \mathrm{~h}$ delay) (total of 48 individual experiments) and (B) under conditions of optimal discrimination after a $1 \mathrm{~h}$ delay (total of 36 individual experiments). The order of experiments per condition was arranged from lowest to highest $d 2$ value. Some data points in $(\mathbf{A})$ were different from zero ( ${ }^{*}$ one-sample $t$-tests, $p<0.05$ ). No statistically significant differences were found in $(\mathbf{A})$ when they were compared with a FICT group (mean: $0 ;$ SEM: 0.07). In (B) all data points are statistically different from zero (one-sample $t$-test) or when compared with a FICT group. 
First of all, these data show that about half the observed groups had a $d 2$ below zero and half them were above zero. This reflects the notion that, on average, the performance under these conditions approximates the value zero. In two of the 48 individual sessions the $d 2$ value differed significantly from zero (one-sample $t$-tests). When the individual sessions were compared with a FICT group, none of the groups performed significantly above or below chance level. Test sessions applying a $1 \mathrm{~h}$ delay condition, without amnesic treatment, always had $d 2$ values $>0.20$, which were significantly different from zero (one-sample $t$-tests, $p<0.05$ ) and the FICT group (independent samples $t$-test), indicating that animals discriminated between the new and familiar object (see Fig. 1B). These data show that comparisons with zero, using a one sample $t$-test, sometimes can reveal a discrimination performance in conditions where no discrimination performance is expected. These occasional differences were not found if the same groups were compared with a FICT group. Furthermore, these data provide experimental evidence that, although values may vary around zero, under normal testing conditions $d 2$ values are not expected to be statistically lower than zero.

\section{DISCUSSION}

In this study we investigated various aspects of the statistical analysis of data from the ORT. The examples of different analyses that were used in this study were derived from a large original data set and fictive data sets to exemplify effects under certain conditions. The aim of these examples was to give recommendations for the analysis of discrimination measures of ORT testing.

First, the different discrimination measures that are being used in the literature were compared. It was found that the relative discrimination measure $(d 2)$ and the investigation ratio $(d 3)$ behaved statistically identical, when comparing their analyses. However, the $p$-value of the absolute discrimination measure ( $d 1)$ was different from that of the $d 2$ and $d 3$ measures. Although the difference between the absolute discrimination measure $d 1$ and the relative measures $d 2$ and $d 3$ may appear small, it is still a relevant difference since it may lead to contradicting conclusions based on the 
exact same dataset. It was found that, in the fictional examples used in the present study, the $d 1$ was more sensitive in revealing differences between groups as compared to the $d 2$ and $d 3$ parameters. However, this difference was not observed in our experimental data (Akkerman, et al., 2012). The d1 measure was shown to be more vulnerable to differences in exploration levels and SEM. The $d 2$ and $d 3$ measures, which correct for exploratory differences, proved to be more sensitive to subtle group differences, when groups with different exploration levels were compared.

Although under certain circumstances $d 1$ can be more sensitive than $d 2$ or $d 3$, the $d 1$ measure should be used with caution. For example, in case of low exploration levels the $d 1$ measure can reveal a difference from zero whereas the absolute level of $d 1$ is still relatively low (as shown in Table 5), which increases the risk of making a Type I error. Furthermore, when using $d 1$, exploratory differences between experimental groups may prevent the detection of differences in discrimination performance (see Table 6). Relative measures, like $d 2$ and $d 3$ correct for the total exploration and are therefore independent of exploration levels. This is confirmed by studies in which we found that the $d 1$ measure, but not the $d 2$ or $d 3$ measure, was correlated with the absolute exploration levels of the animals in the second trial (Akkerman, et al., 2012; Sik, et al., 2003). Taken together, these findings suggest that the $d 2$ or $d 3$ measures should be preferred in case of low exploration or group differences in exploration levels.

In order to interpret discrimination performance in the ORT, it is suggested to make two comparisons. First, it should be analyzed whether the discrimination measure of the animals in each treatment condition differs from zero (or in case of $d 3,0.5$ ), to assess whether or not memory is really present in a condition. Furthermore, it should be analyzed whether a treatment condition differs from the control condition, to assess whether the treatment has either improved or impaired discrimination performance. This manner of analyzing data allows a more differentiated manner for analyzing the data. 
When testing whether animals do discriminate between the objects, it is not recommended to compare the discrimination performance with chance level performance $(0$ or 0.5 ) by means of a one-sample $t$-test. Because by doing so, a comparison is made with an assumed population mean of zero (or 0.5) without variance. This may increase the likelihood of making a Type I error, as shown in Fig. 1. In the present study it was proposed that a FICT group could be constructed with a mean discrimination ( $d 1$ and $d 2$ ) performance of zero and with a realistic standard deviation that is derived from representative experimental groups. This corresponds well with the experimental data pooled in Fig. 1A, where no discrimination was expected. Although the $d 2$ value of individual test sessions in Fig. 1A varied, the average $d 2$ value was zero. Of note, there is no such thing as a standard FICT group. An appropriate FICT group should be constructed for each individual experimental study, based on number of animals tested and variance of real experimental groups. The mean should be set on 0 (or 0.5 for $d 3$ ) based on the rationale behind the ORT paradigm. A realistic standard deviation could be calculated after performing an experiment, based on standard deviations found in the experimental groups. Taken together, comparison with a FICT group can be considered to be a more realistic alternative to examine whether experimental groups perform above chance level than comparisons with a fixed value.

The observation that there are natural fluctuations in the discrimination performance indicates that one should be cautious when making comparisons with a control group that does not discriminate between the objects (see Table 4B). If the control group has a negative $d 2$ value (but not different from zero), it is much more likely to find a 'complete effect' as compared to a control group that has a slight positive value (also not different from zero). The latter condition may be result in an 'intermediate effect' or even no effect, while a negative control group may exaggerate the effect. The variance that may occur in a single study (a sample) may therefore lead to erroneous conclusions. This can be prevented by referring to a FICT group when testing whether a treatment has an intermediate or complete effect. 
In the present study it was shown that, after examination of the 48 different ORT sessions from our lab, in which no memory was expected, the relative discrimination performance $(d 2)$ was not found to deviate significantly from zero when compared with a FICT group. Also, many other studies have not shown discrimination performance levels lower than zero (J. P. Aggleton, Blindt, \& Rawlins, 1989; Blokland, Prickaerts, Honig, \& De Vente, 1998; Bussey, Muir, \& Aggleton, 1999; Dodart, Mathis, \& Ungerer, 1997; Ennaceur \& Aggleton, 1994; Gaskin, et al., 2003; Lieben, et al., 2005; Pitsikas, Rigamonti, Cella, \& Muller, 2002; Winters \& Bussey, 2005). Aside from these experimental data showing that discrimination performance is not expected to drop below zero, this can also be based on a conceptual level. The basic assumption in object recognition testing is that, if rats do not remember the familiar object they will not discriminate between the objects. Under proper testing conditions, there is no reason to expect that rats have a greater preference for the familiar object in the test trial. Only under specific conditions a preference for the familiar object may arise, which could lead to higher exploration levels to the familiar object and consequently to negative discrimination performance. That is, if a familiar object is physically more interesting to the animals (as compared to the novel object) or if the animal is drawn to the part of the arena where the familiar object is located. Both phenomena can be prevented by proper experimental design. When object- and place preferences are absent (or accounted for), one can theoretically assume that the discrimination performance will only be in between 0 and 1. Therefore, under proper testing conditions one-sided testing to investigate whether the discrimination measure is higher than zero should be allowed, which would increase the power of the statistical test and reduce the number of animals required.

The present study gave specific examples of how data of the ORT can be analyzed. It was shown that differences in exploration levels, and especially low levels of exploration, may affect the statistical analysis and therefore the reliability of the absolute discrimination measure $d 1$. Therefore, the use of $d 2$ or $d 3$ is recommended since these measures are more robust against differences in exploration. Further, it was shown that a FICT group with a mean discrimination performance $(d 2)$ of zero and a variance based on prior experimental data may be the most valid method to test 
whether individual experimental groups discriminate between objects. Finally, it was shown that under optimal test conditions (i.e. no side- or object preference), onesided testing is allowed. 


\section{REFERENCES}

Aggleton, J. P. (1985). One-trial object recognition by rats. The Quarterly Journal of Experimental Psychology, 37B, 279-294.

Aggleton, J. P., Blindt, H. S., \& Rawlins, J. N. (1989). Effects of amygdaloid and amygdaloid-hippocampal lesions on object recognition and spatial working memory in rats. Behavioural Neuroscience, 103, 962-974.

Akkerman, S., Blokland, A., Reneerkens, O., van Goethem, N. P., Bollen, E., Gijselaers, H. J., et al. (2012). Object recognition testing: Methodological considerations on exploration and discrimination measures. Behav Brain Res, 232(2), 335347.

Blokland, A., Prickaerts, J., Honig, W., \& De Vente, J. (1998). State-dependent impairment in object recognition after hippocampal NOS inhibition. NeuroReport, 9, 4205-4208.

Bussey, T., Muir, J. L., \& Aggleton, J. P. (1999). Functionally dissociating aspects of event memory: the effects of combined perirhinal and postrhinal cortex lesions on object and place memory. The Journal of Neuroscience, 19, 495502.

Dodart, J. C., Mathis, C., \& Ungerer, A. (1997). Scopolamine-induced deficits in a two-trial object recgnition task in mice. NeuroReport, 8, 1173-1178.

Ennaceur, A., \& Aggleton, J. P. (1994). Spontaneous recognition of object configurations in rats: effects of fornix lesions. Experimental Brain Research, 100(1), 85-92.

Ennaceur, A., \& Delacour, J. (1988). A new one-trial test for neurobiological studies of memory in rats. 1: behavioral data. Behavioural Brain Research, 31, 47-59.

Ennaceur, A., Michalikova, S., Bradford, A., \& Ahmed, S. (2005). Detailed analysis of the behavior of Lister and Wistar rats in anxiety, object recognition and object location tasks. Behavioral Brain Research, 159, 247-266.

Gaskin, S., Tremblay, A., \& Mumby, D. G. (2003). Retrograde and anterograde object recognition in rats with hippocampal lesions. Hippocampus, 13, 962-969.

Kertesz, S., Kapus, G., Gacsalyi, I., \& Levay, G. (2010). Deramciclane improves object recognition in rats: potential role of NMDA receptors. Pharmacology Biochemistry and Behavior, 94, 570-574.

King, M. V., Sleight, A. J., Woolley, M. L., Topham, I. A., Marsden, C. A., \& Fone, K. C. (2004). 5-HT6 receptor antagonists reverse delay-dependent deficits in novel object discrimination by enhancing consolidation--an effect sensitive to NMDA receptor antagonism. Neuropharmacology, 47, 195-204.

Lieben, C. K., Blokland, A., Sik, A., Sung, E., van Nieuwenhuizen, P., \& Schreiber, R. (2005). The selective 5-HT(6) receptor antagonist Ro4368554 restores 
memory performance in cholinergic and serotonergic models of memory deficiency in the rat. Neuropsychopharmacology, 30, 2169-2179.

Messier, C. (1997). Object recognition in mice: improvement of memory by glucose. Neurobiology of Learning and Memory, 67, 172-175.

Pichat, P., Bergis, O. E., Terranova, J. P., Urani, A., Duarte, C., Santucci, V., et al. (2007). SSR180711, a novel selective alpha7 nicotinic receptor partial agonist: (II) efficacy in experimental models predictive of activity against cognitive symptoms of schizophrenia. Neuropsychopharmacology, 32, 17-34.

Pitsikas, N., Rigamonti, A. E., Cella, S. G., \& Muller, E. E. (2002). Effects of the nitric oxide donor molsidomine on different memory components as assessed in the object-recognition task in the rat. Psychopharmacology, 162, 239-245.

Prickaerts, J., Sik, A., Van Der Staay, F. J., De Vente, J., \& Blokland, A. (2005). Dissociable effects of acetylcholinesterase inhibitors and phosphodiesterase type 5 inhibitors on object recognition memory: acquisition versus consolidation. Psychopharmacology, 177, 381-390.

Rutten, K., Lieben, C., Smits, L., \& Blokland, A. (2007). The PDE4 inhibitor rolipram reverses object memory impairment induced by acute tryptophan depletion in the rat. Psychopharmacology, 192, 275-282.

Rutten, K., Prickaerts, J., Hendrix, M., van der Staay, F. J., Sik, A., \& Blokland, A. (2007). Time-dependent involvement of CAMP and CGMP in consolidation of object memory: Studies using selective phosphodiesterase type 2, 4 and 5 inhibitors. European Journal of Pharmacology, 558, 107-112.

Rutten, K., Prickaerts, J., Schaenzle, G., Rosenbrock, H., \& Blokland, A. (2008). Subchronic rolipram treatment leads to a persistent improvement in long-term object memory in rats. Neurobiology of Learning and Memory, 90, 569-575.

Sik, A., van Nieuwehuyzen, P., Prickaerts, J., \& Blokland, A. (2003). Performance of different mouse strains in an object recognition task. Behavioural Brain Research, 147, 49-54.

Snigdha, S., \& Neill, J. C. (2008). Improvement of phencyclidine-induced social behaviour deficits in rats: involvement of 5-HT1A receptors. Behavioural Brain Research, 191, 26-31.

Winters, B. D., \& Bussey, T. J. (2005). Transient inactivation of perirhinal cortex disrupts encoding, retrieval, and consolidation of object recognition memory. Journal of Neuroscience, 25, 52-61. 

Chapter 4 


\section{PDE5 INHIBITION IMPROVES ACQUISITION PROCESSES AFTER}

\section{LEARNING VIA A CENTRAL MECHANISM}

S. Akkerman, A. Blokland, N.P. van Goethem, P. Cremers, C.L. Shaffer, S.M. Osgood, H.W.M. Steinbusch and J. Prickaerts 


\section{ABSTRACT}

In previous studies, we have shown that phosphodiesterase type 5 inhibitors (PDE5-Is) can improve early consolidation of object memory. These conclusions were based on the timing of drug administration relative to the learning trial (i.e. before or after). However, there are very little pharmacological data available about the pharmacokinetic profile of orally administered PDE5-Is in the rat. Furthermore, there is still debate whether these effects are achieved via central or peripheral mechanisms and if acquisition processes are improved. In the current study, we tested the effects of the PDE5-I vardenafil in a cholinergic-deficit model and compared the effects after intracerebroventricular (ICV) versus oral (PO) administration. We found that PO vardenafil restored a scopolamine-induced memory impairment when dosed within 2 min after the learning trial while ICV vardenafil was able to restore memory when injected within 4 min after learning. Because the test trial was within 10 min after the learning trial, this suggests that these effects on object memory are related to acquisition processes that may still be ongoing in a time window after the learning trial. To further elucidate the extent of this acquisition window, we investigated the pharmacokinetic profile of vardenafil after PO administration where it was detected within 4 min post-dose. Taken together, our data suggest that PDE5 is involved in acquisition processes, which may linger for at least 4-6 min after learning. Further studies are needed to exclude that these effects could also be explained on basis of an effect on early consolidation processes. Additionally, the effectiveness of ICVadministered vardenafil provides further experimental evidence that PDE5-Is improve memory via a central mechanism. 


\section{INTRODUCTION}

Memory is an important cognitive function that is founded on an intricate system of neurobiological processes, which comprise a variety of neurotransmitters and intracellular messenger molecules that are differentially involved in specific stages of memory formation (Izquierdo, et al., 2006). Memory stages, like acquisition and early consolidation, are considered to be temporally and mechanistically distinct. For example, acetylcholine is an important neurotransmitter involved in the initial acquisition processes. Studies have shown that blockade of the muscarinic acetylcholine receptors specifically impairs memory acquisition (Gil-Bea, et al., 2011; Rasch, Born, \& Gais, 2006; Robinson, Platt, \& Riedel, 2011). Alternatively, the intracellular messenger cyclic guanosine monophosphate (cGMP) is supposed to be primarily involved in early consolidation, which starts immediately after the initial stimulus and last up to 1-3 h (Bernabeu, Schmitz, Faillace, Izquierdo, \& Medina, 1996; Bernabeu, et al., 1997; Prickaerts, de Vente, Honig, Steinbusch, \& Blokland, 2002; Reymann \& Frey, 2007; Rutten, et al., 2007). However, cGMP may also be involved in acquisition processes, as pharmacologically increasing cGMP levels has been effective in overcoming cholinergic acquisition deficits, likely by increasing neurotransmitter concentrations including glutamate release (Devan, et al., 2004; Reneerkens, et al., 2012).

Intracellular CGMP concentrations are regulated by enzymes called phosphodiesterases (PDEs). There are 11 families of PDEs (PDE1-11) that differ in localization and affinity for CGMP and/or CAMP (Bender \& Beavo, 2006). A PDE that specifically degrades CGMP is PDE5. In humans, reasonable amounts of PDE5 mRNA have been detected virtually throughout the entire body (Kotera, Fujishige, \& Omori, 2000; Lin, 2004; Stacey, Rulten, Dapling, \& Phillips, 1998). It is also present in the human central nervous system: abundantly in the cerebellum and to a lower extent in the rest of the brain (Foresta, et al., 2008; Lin, 2004). In rats, PDE5 mRNA expression has been detected in hippocampus (CA1-3, dendate gyrus and subiculum), putamen, forebrain, hypothalamus, and various cortical regions (including the piriform-, cingulate- and 
entorhinal cortex) with the highest central concentrations in the cerebellum (Kotera, et al., 2000; Van Staveren, et al., 2003).

PDEs can be inhibited by a class of drugs called PDE-inhibitors (PDE-Is). For this reason several PDE5-specific inhibitors, like sildenafil, vardenafil and tadalafil, have been successfully developed for the treatment of erectile dysfunction and pulmonary hypertension (Ghofrani \& Grimminger, 2006). However, PDE5-Is are also under investigation for their therapeutic potential as cognitive enhancers, as higher neuronal cGMP levels support LTP (Blokland, Schreiber, \& Prickaerts, 2006; Ghofrani, Osterloh, \& Grimminger, 2006; Puzzo, Sapienza, Arancio, \& Palmeri, 2008; Reneerkens, Rutten, Steinbusch, Blokland, \& Prickaerts, 2009).

Although many animal studies have shown that PDE5-Is are capable of enhancing memory, it is still debatable whether these effects are exerted via central neuronal mechanisms or related to the important role of PDE5 and CGMP in the regulation of vascular tone (i.e. enhanced circulation). The latter is a plausible explanation since PDE5 is highly expressed in the vascular beds, whereas expression in the brain structures is relatively low. Furthermore, only 2 (PDE5 A1 and PDE5 A2) of the 3 PDE5 splice variants (PDE5 A1-3) are expressed in the central nervous system (Lin, 2004). Human studies have resulted in somewhat controversial findings about whether or not cerebral blood flow is affected by PDE5-Is (Arnavaz, et al., 2003; Rosengarten, et al., 2006). In rats, no changes in cerebral blood flow were found after orally administered vardenafil that improved memory performance; instead, glucose utilization was found to be decreased (Rutten, et al., 2009). Yet, it is still ambiguous if pro-cognitive effects of PDE5-Is can be explained by central mechanisms, since administration of the non-brain-penetrant PDE5-I UK-343,664 was able to improve memory in a scopolamine-induced cholinergic deficit model (Reneerkens, et al., 2012).

In the present study we used a different approach to gain further insight into the effects of PDE5 inhibition on acquisition processes. We applied the scopolamine model to induce an acquisition deficit and used short test delays (10 min and $1 \mathrm{~h}$ ) to exclude the involvement of consolidation processes. To gain insight into the temporal characteristics of cGMP signaling shortly after learning and the possible involvement 
of cGMP in acquisition processes, we administered the PDE5 inhibitor at different short intervals after the acquisition trials. Additionally, we investigated whether cognition enhancement by a PDE5-I can be explained by a central mechanism. Therefore, we investigated the neuropharmacokinetic profile (plasma and brain) of vardenafil after PO dosing. Further, we compared two different administration routes of vardenafil. Vardenafil was administered either intracerebroventricularly (ICV) or orally $(\mathrm{PO})$ and related these to the pharmacokinetic findings.

\section{Methods}

\section{ANIMALS}

Four groups of 4-month-old male Wistar rats were ordered from Harlan Laboratories B.V. (Horst, the Netherlands). Sixteen animals were used for testing the ICV conditions and two other groups of 16 and 24 animals, respectively, were used for the PO conditions of the experiment. Finally, a group of 28 animals was used for pharmacokinetic determinations. Rats were housed individually in standard Makrolon TM Type III cages on sawdust bedding in an air-conditioned room (about $20^{\circ} \mathrm{C}$ ) with a radio always playing softly in the background. They were kept under a reversed 12/12-hour light/dark cycle (lights on from 7 PM to 7 AM). Food and water were provided ad libitum. The animals were housed and tested in the same room.

\section{TREATMENT}

Test solutions were always freshly prepared at the start of each experimental day. Vardenafil (100\% chemical purity, MW of $448.6 \mathrm{ng} / \mathrm{nmol}$ ) was kindly donated by Bayer AG, Wuppertal, Germany. Vardenafil was chosen as it is (about 5 times) more potent than sildenafil both at the level of IC50 (Kim, Huang, Goldstein, Bischoff, \& Trais, 2001) and behavior (see Prickaerts, van Staveren, Sik, Markerink-van Ittersum, Niewohner, et al., 2002). The vehicle for PO administered vardenafil consisted of tylose $(0.5 \%$ methyl-cellulose) solution for $98 \%$ and $2 \%$ of Tween 80 . ICV administered vardenafil was suspended in saline $(0.9 \% \mathrm{NaCl})$. Vardenafil was 
administered either PO (1 mg/kg, $1 \mathrm{ml} / \mathrm{kg})$ or ICV) $(30 \mu \mathrm{g}$ in $1 \mu \mathrm{l})$, by bolus injection at varying time points shortly after $\mathrm{T} 1$. For $\mathrm{PO}$ administrations, the animals were loosely restrained by holding the skin on the back of the neck and the solution was injected into the stomach using a bulb-tipped gastric gavage. In order to perform ICV injections, rats first received a guide cannula. Animals were fully anesthetized with isoflurane (induction 5\%, maintenance $2 \%$ ) and fixed in a stereotaxic frame, and a guide cannula was placed above the left lateral ventricle at the following coordinates with reference to bregma: $0.8 \mathrm{~mm}$ posterior, $1.55 \mathrm{~mm}$ lateral and $3.8 \mathrm{~mm}$ ventral (Paxinos \& Watson, 1996). The tip of the cannula ended $1.0 \mathrm{~mm}$ above the lateral ventricle. The cannula was fixed to the skull using acrylic dental cement and two small screws. After surgery, the animals were allowed to recover for 2 weeks. During testing, an injection needle, which was $1.0 \mathrm{~mm}$ longer than the guide cannula, was inserted into the guide cannula to deliver vardenafil into the lateral ventricle. The needle was left in place for an additional minute to prevent the reflux of infused vardenafil. Scopolamine was obtained from Sigma Aldrich Chemie B.V. (Zwijndrecht, the Netherlands) and dissolved in saline. Scopolamine $(0.1 \mathrm{mg} / \mathrm{kg}, 1 \mathrm{ml} / \mathrm{kg})$ was always administered intraperitoneally (IP) 30 min before T1. The PO dose of vardenafil and IP dose of scopolamine were determined based on earlier studies using these drugs (Rutten, Prickaerts, \& Blokland, 2006; Rutten, et al., 2007; Rutten, Prickaerts,

Schaenzle, Rosenbrock, \& Blokland, 2008). Based on pilot data (not shown), a dose of $30 \mu \mathrm{g}$ vardenafil was used for ICV administration.

\section{THE OBJECT RECOGNITION TASK}

The object recognition task (ORT) was based on the original version described by Ennaceur and Delacour (Ennaceur \& Delacour, 1988). The test was performed in a circular arena, $83 \mathrm{~cm}$ in diameter. The floor of the apparatus and half of the $40 \mathrm{~cm}$ high arena wall was made of gray polyvinyl chloride (PVC) and the front-half (facing the observer) consisted of transparent PVC. Two objects were placed at the mid-line between the gray and transparent walls, at symmetrical positions, about $10 \mathrm{~cm}$ from the wall. Each object was available in triplicate. Four different sets of identical objects were systematically rotated over the individual animals. The different objects were: 1) 
a cone consisting of a gray PVC base (maximal diameter of $18 \mathrm{~cm}$ ) with a collar on top made of aluminum (total height of $16 \mathrm{~cm}$ ), 2) a standard 1 liter transparent glass bottle (diameter $10 \mathrm{~cm}$, height $22 \mathrm{~cm}$ ) filled with water, 3) a massive metal cube $(10.0$ $\times 5.0 \times 7.5 \mathrm{~cm}$ ) with two holes (diameter of $1.9 \mathrm{~cm}$ ), and 4) a solid aluminum cube with a tapered top $(13.0 \times 8.0 \times 8.0 \mathrm{~cm})$. Rats were unable to displace the objects. For more detailed information about the apparatus and test procedure see Akkerman et al. (2012).

Individual animals performed multiple test sessions over the course of the experiment. A single testing session consisted of two 3 min trials, a learning trial (T1) and a test trial (T2), separated by a retention interval. At the beginning of each trial, rats were placed in the front of the arena, facing the transparent wall. During T1 the apparatus contained two identical objects, or samples $\left(a_{1}\right.$ and $\left.a_{2}\right)$, which the rat could freely explore. After $\mathrm{T} 1$ the animal was returned to its home cage for the duration of the retention interval $(10 \mathrm{~min}$ or $24 \mathrm{~h}$ ). After the retention interval the rat was reintroduced to the arena for T2. However, in T2 the objects from T1 were replaced by a copy of the samples $\left(a_{3}\right)$ and a novel object (b). In $\mathrm{T} 2$, the animal was again allowed to freely explore both objects for $3 \mathrm{~min}$.

The time animals spent exploring the individual objects during each trial was recorded manually with a personal computer. Exploration was defined by directing the nose to the object at a maximal distance of $2 \mathrm{~cm}$ and/or touching the object with the nose. Sitting on the object was not considered exploratory behavior. As animals require a minimal amount of object interaction to achieve reliable object discrimination, rats that explored less than $6 \mathrm{~s}$ in T1 and/or $9 \mathrm{~s}$ in T2 were excluded from the analyses (Akkerman, et al., 2012; Sik, van Nieuwehuyzen, Prickaerts, \& Blokland, 2003). Objects were cleaned with a $70 \%$ ethanol solution before each trial to avoid the presence of olfactory cues.

\section{EXPERIMENTAL PROCEDURE AND TREATMENT CONDITIONS}

After surgery the ICV animals were allowed to recover for 2 weeks. Next, all animals were habituated to the apparatus, test procedure and drug administration (Akkerman, 
et al., 2012). When the animals were fully habituated, the experiments exploring the route of vardenafil administration were tested. A wash-out period of at least one full day was always interposed between test sessions.

In all experiments, scopolamine was dosed 30 min before T1. Subsequently, PO $(n=$ 16 per condition) or ICV ( $n=16$ per condition) administration of vardenafil were tested in combination with a scopolamine-induced memory impairment using a 10 min retention interval. The scopolamine model was tested by injecting either saline or scopolamine (30 min before T1) in combination with the vehicle of vardenafil ( 0 min after T1). Vardenafil was tested at different post-T1 injection intervals: 0, 2, 4 and 6 min, and 8 min only in the ICV experiment. Finally, PO administered vardenafil was also tested in a $1 \mathrm{~h}$ interval ( $\mathrm{n}=8$ per condition), using the dosing time points of 0,2 , 4, 15, and 30 min after T1. We applied these short intervals in order examine whether the treatments affected acquisition-like processes and not consolidation processes per se. In previous studies with PDE inhibitors in the ORT it has been shown that the latter processes take place more than $1 \mathrm{~h}$ after acquisition (Rutten et al., 2007).

\section{PHARMACOKINETIC MEASUREMENTS}

Twenty-eight rats were used for the determination of plasma and brain vardenafil concentrations over the first hour following its oral administration. At 2, 4, 6, 10, 20, 30 and 60 min post-dose, 4 animals were sacrificed for blood and brain sampling. Blood was drawn from the saphenus vein using heparin-coated tubes, which were temporarily stored on wet ice, and immediately after blood collection the animal was decapitated. The complete brain was collected, rinsed with ice-cold saline, placed in a cup, weighed, and immediately frozen on dry ice. Plasma was isolated using centrifugation ( $1500 \mathrm{G}$ for $10 \mathrm{~min}$ at $4^{\circ} \mathrm{C}$ ) and pipetted into eppendorf vials. Plasma and brain samples were stored at $-80{ }^{\circ} \mathrm{C}$ until bioanalytical processing. Vardenifil quantification within collected plasma and brain was performed at BioDuro, Pharmaceutical Product Development Inc. (Shanghai, PRC). For bioanalytical sample preparation, plasma was used as is, while brain samples were first homogenized in a 4-fold volume (w/v) of water. Both matrices were processed for drug quantification using liquid-liquid extraction methodology followed by a characterized liquid 
chromatography-tandem mass spectrometry (LC-MS/MS) assay. Standard curves were prepared in control matrices, an appropriate dynamic range was achieved, and instrument settings and potentials were adjusted to optimize the MS signal for vardenafil. Raw data were processed using Analyst Software version 1.4.2 (AB Sciex Inc., Ontario, Canada). The lower limit of quantification (LLOQ) was $0.05 \mathrm{ng} / \mathrm{ml}$ for plasma and $0.01 \mathrm{ng} / \mathrm{g}$ for brain.

\section{STATISTICAL ANALYSIS}

The readout parameters of the ORT are the amount of time that rats spent exploring each object, i.e. the two identical objects a1 and a 2 in $\mathrm{T} 1$, and in $\mathrm{T} 2 \mathrm{a} 3$ and b. From these exploration times the following variables were calculated: $e 1=a_{1}+a_{2}, e 2=a_{3}+$ $b$, and $d 2=\left(b-a_{3}\right) / e 2$ (see Table 1). e1 and e2 represent the total exploration time of both objects during $T 1$ and $T 2$, respectively. The $d 2$ index is a relative measure of discrimination (ranging between -1 and 1), corrected for total exploration activity in the test-trial (e2) and is an indication of memory performance (Akkerman, et al., 2012). A $d 2$ value higher than zero means that animals spent significantly more time exploring the novel object in $\mathrm{T2}$, which is an indication that object memory was present. One-sample $t$-tests were performed on each treatment to assess whether $d 2$ was different from zero. Because the comparison of the mean $d 2$ value with the fixed value zero may not be the most valid way for analyzing recognition (increased chance of making a type I error), the treatments were also compared using one-way ANOVA. If ANOVA indicated significant differences between the treatment conditions, then post-hoc analyses were performed comparing each vardenafil treatment to the saline and the scopolamine reference treatments using Dunnett $t$-tests.

Table 1 Measures involved in the ORT

\begin{tabular}{cl}
\hline Exploration & Discrimination \\
\hline$e 1=a_{1}+a_{2}$ & $d 2=\left(b-a_{3}\right) / e 2$ \\
$e 2=a_{3}+b$ & $d(a)$ and
\end{tabular}

e1 is the measure of the time spent in exploring both identical objects (a1 and a2) in the first trial, and e2 is the measure of the time spent in exploring both the familiar (a) and new object (b) in the second trial. $d 2$ corresponds to the ability to discriminate between the old and new object during the second trial and is corrected for exploration time during that trial. 


\section{RESULTS}

\section{EFFECTS OF VARDENAFIL IN A CHOLINERGIC DEFICIT MODEL - 10 MIN RETENTION INTERVAL}

Animals performed the ORT with a retention interval of $10 \mathrm{~min}$, in combination with scopolamine treatment. Two different administration routes for vardenafil were tested, PO (Figure 1) and ICV (Figure 2), and each was tested at different dosing time points shortly after the learning trial (T1). First, the scopolamine model was tested for each experiment with saline and scopolamine treatments 30 min before T1 (both in combination with the vardenafil vehicle injected directly after T1).

Within the PO and ICV experiment, total exploration levels were compared between the treatment conditions. In the PO experiment, we found no differences in the level of exploration during $T 1(F(5,75)=0.71$, n.s. $)$ and $T 2(F(5,75)=1.47$, n.s. $)$. Similarly in the ICV experiment, no significant differences were present during $T 1(F(6,76)=2.09$, n.s.) and $\mathrm{T} 2(F(6,76)=2.06$, n.s. $)$.

One sample $t$-tests were used to compare the $d 2$ value of each treatment condition to zero. In contrast to the scopolamine condition, the saline condition of the PO experiment had a $d 2$ value that was significantly higher than zero. This was the same for the ICV experiment. These results indicate that, in both experiments, saline-treated animals were able to successfully discriminate between objects after a $10 \mathrm{~min}$ retention interval and that scopolamine effectively impaired object discrimination. When vardenafil was given $\mathrm{PO}, d 2$ was only higher than zero when the drug was injected within 2 min after T1. When PO injections were given 4 or 6 min after T1 the $d 2$ value was not statistically different from zero (Figure 1). Alternatively, when vardenafil was administered ICV, $d 2$ was higher than zero when the injection took place up to $4 \mathrm{~min}$ after $\mathrm{T} 1$. Object discrimination was no longer present when vardenafil was injected 6 min after T1 (Figure 2). 


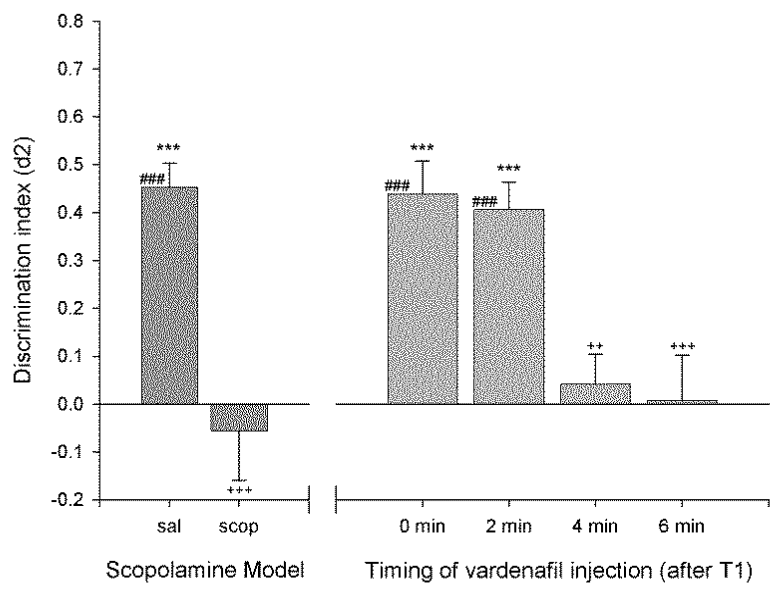

\section{Figure 1 ORT 10 min interval - vardenafil PO}

Memory performance of rats with a scopolamine-induced memory acquisition deficit in the Object Recognition Task (ORT), with a 10 min retention interval. Scopolamine was always administered 30 min prior to T1. In the control conditions scop $(n=11)$ and sal $(n=15)$, vehicle was administered directly (0 min) after T1. Vardenafil $(1 \mathrm{mg} / \mathrm{kg}, \mathrm{PO})$ was administered at $0(n=13), 2(n=15), 4(n=13)$ or $6 \mathrm{~min}(n=14)$ after the learning trial (T1). Discrimination performance (d2) was compared to zero (one-sample t-tests; \#\#\#: $p<$ 0.001 ), scopolamine (scop) treatment (Dunnett t-tests; $* * *: p<0.001)$ and saline (sal) treatment (Dunnett t-tests; ++: $p<0.01 ;+++: p<0.001$ ). The reference treatments (saline and scopolamine) are shown in dark grey and the vardenafil treatments are shown in light gray.

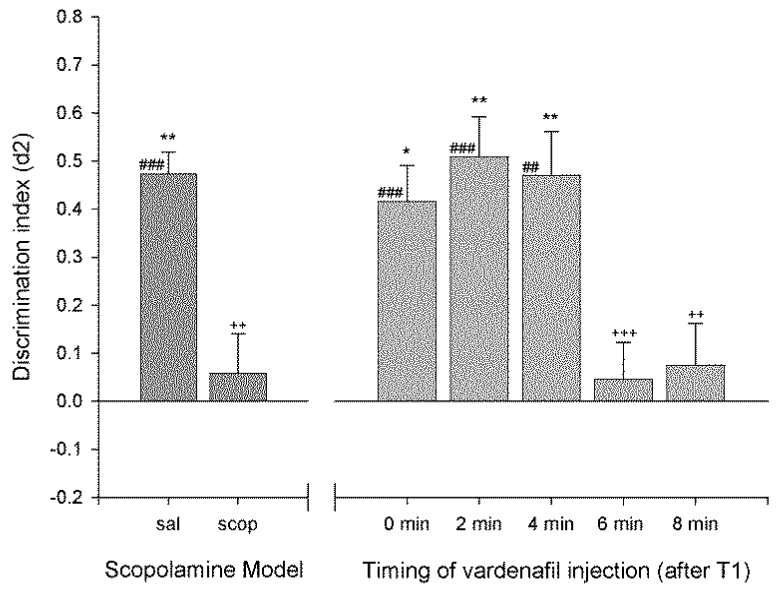

\section{Figure 2 ORT 10 min interval - vardenafil ICV}

Memory performance of rats with a scopolamine-induced memory acquisition deficit in the Object Recognition Task (ORT), with a 10 min retention interval. Scopolamine was always administered 30 min prior to T1. In the control conditions $\operatorname{scop}(n=10)$ and sal $(n=16)$, vehicle was administered directly (0 min) after T1. Vardenafil $(30 \mu \mathrm{g}, \mathrm{ICV})$ was administered $0(n=10), 2(n=10), 4(n=9), 6(n=15)$ or $8(n=13)$ min after the learning trial (T1). Discrimination performance (d2) was compared to zero (one-sample $t$-tests; \#\#: $p<0.01$; \#\#\#: $p<0.001$ ), scopolamine (scop) treatment (Dunnett $t$-tests; ${ }^{*}: p<0.05 ;{ }^{* *}: p<0.01$ ) and saline (sal) treatment (Dunnett $t$-tests; $++: p<0.01 ;+++: p<0.001$ ). The reference treatments (saline and scopolamine) are shown in dark grey and the vardenafil treatments are shown in light gray. 
One-way ANOVA revealed that significant $d 2$ differences were present between treatment conditions in the PO experiment $(F(5,75)=10.79, p<0.001)$ as well as the ICV experiment $(F(6,76)=8.92, p<0.001)$. In the PO experiment, post-hoc analyses using Dunnett $t$-tests showed that the $d 2$ values of the 0 and 2 min conditions were equal to that of the saline condition and significantly higher than that of the scopolamine condition (Figure 1). The same was found for the $d 2$ of the 0,2 and 4 min conditions of the ICV experiment (Figure 2). All other vardenafil treatment conditions had $d 2$ values that were equal to that of the scopolamine condition and significantly lower than that of the saline condition. These results show that vardenafil was able to fully restore object discrimination when given PO within 2 min or ICV within 4 min after T1.

\section{EFFECTS OF VARDENAFIL IN A CHOLINERGIC DEFICIT MODEL $-1 \mathrm{H}$ RETENTION INTERVAL}

In this experiment the retention of the ORT was extended to $1 \mathrm{~h}$ and vardenafil was administered PO. As in the 10 min retention experiments, the two reference conditions (saline and scopolamine, both in combination with vardenafil vehicle treatment) were first tested. The results of the $1 \mathrm{~h}$ retention experiment are graphically presented in Figure 3.

Exploration levels during T1 and T2 were compared using one-way ANOVA. As in the 10 min retention experiments, no significant differences were found in exploration levels during $\mathrm{T} 1(F(6,49)=1.22$, n.s. $)$ and $T 2(F(6,49)=0.26$, n.s. $)$ between the different treatment conditions in the $1 \mathrm{~h}$ retention experiment.

One sample $t$-tests showed that the saline condition had a $d 2$ value significantly higher than zero whereas the $d 2$ value of the scopolamine condition was not, indicating that saline-treated animals were able to successfully discriminate between objects after a $1 \mathrm{~h}$ retention interval and that scopolamine effectively impaired object discrimination. Identical to the 10 min retention interval with PO testing, the $d 2$ of vardenafil-treated animals was significantly higher than zero when dosing took place 
within 2 min after T1. The $d 2$ was no longer higher than zero when drug administration was performed later than 2 min after T1 (Figure 3).

One-way ANOVA revealed significant differences in $d 2$ measures between the different treatment conditions $(F(6,49)=6.79, p<0.001)$. Post-hoc analyses with Dunnett $t$-tests showed that the $d 2$ values of the 0 and 2 min conditions were equal to that of the saline condition and significantly higher than the $d 2$ of the scopolamine condition. The exact opposite was found for the 4, 15 and 30 min vardenafiltreatment conditions where the $d 2$ values of these conditions were equal to that of scopolamine and significantly lower than that of saline (Figure 3 ). These results show that, identical to the $10 \mathrm{~min}$ retention experiment, vardenafil fully restored object discrimination when given PO within 2 min after T1.

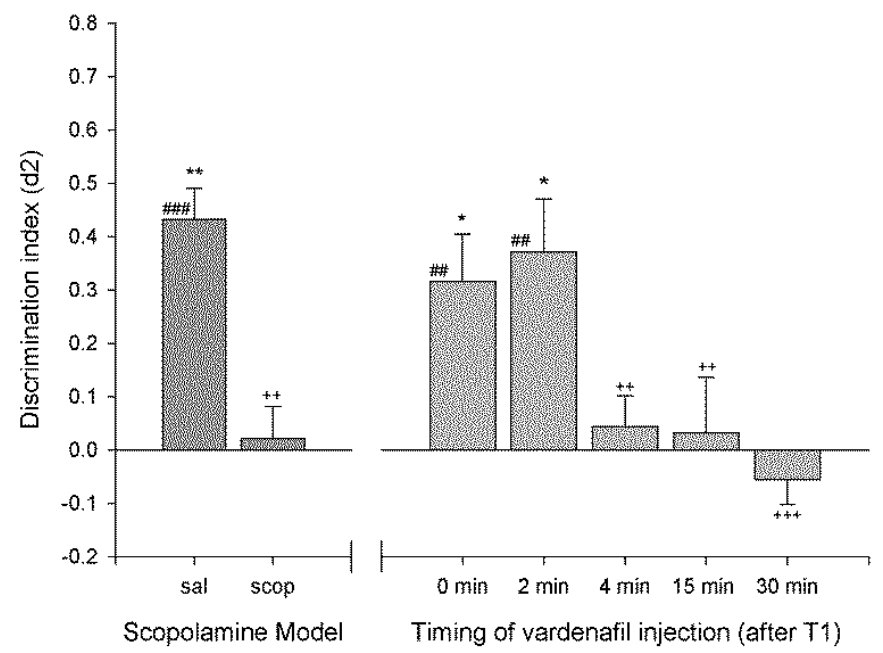

Figure 3 ORT 1h interval - vardenafil PO

Memory performance of rats with a scopolamine-induced memory acquisition deficit in the Object Recognition Task (ORT), with a $1 \mathrm{~h}$ retention interval. Scopolamine was always administered 30 min prior to T1. In the control conditions $\operatorname{scop}(n=8)$ and sal $(n=8)$, vehicle was administered directly $(0 \mathrm{~min})$ after T1. Vardenafil $(1 \mathrm{mg} / \mathrm{kg}, \mathrm{PO})$ was administered $0(n=8), 2(n=8), 4(n=8), 15(n=8)$ or $30(n=8) \mathrm{min}$ after the learning trial (T1). Discrimination performance (d2) was compared to zero (one-sample t-tests; \#\#: $p<0.01$; \#\#\#: $p<0.001)$, scopolamine (scop) treatment (Dunnett $t$-tests; $*: p<0.05 ; * *: p<0.01$ ) and saline (sal) treatment (Dunnett $t$-tests; $++: p<0.01 ;+++: p<0.001$ ). The reference treatments (saline and scopolamine) are shown in dark grey and the vardenafil treatments are shown in light gray. 


\section{VARDENAFIL EXPOSURE-TIME RELATIONSHIPS IN RATS FOLLOWING PO ADMINISTRATION}

Pharmacokinetic data ( $N=4 /$ time point) are shown in Table 1. Vardenafil was $>$ LLOQ in both plasma and brain, 4 minutes after PO injection. The unbound brain/plasma ration varied between 1.61 (4 min) to 0.45 (20 min) and unbound brain and plasma levels of vardenafil reached IC50 values around 10 min after oral administration.

Table 2 Pharmacokinetic measurements of vardenafil (1 mg/kg, PO) in the rat istration

\begin{tabular}{|c|c|c|c|c|c|c|c|}
\hline \multirow[t]{2}{*}{ Parameter } & \multicolumn{7}{|l|}{ Time (min) } \\
\hline & 2 & 4 & 6 & 10 & 20 & 30 & 60 \\
\hline$C_{b}(\mathrm{ng} / \mathrm{g})$ & $0.03(0.03)$ & $0.20(0.07)$ & $0.27(0.08)$ & $1.17(0.48)$ & $1.85(0.20)$ & $1.59(0.35)$ & $1.44(0.05)$ \\
\hline$C_{b, u}(n M)$ & $0.00(0.00)$ & $0.02(0.01)$ & $0.02(0.01)$ & $0.10(0.04)$ & $0.15(0.02)$ & $0.13(0.03)$ & $0.12(0.00)$ \\
\hline$C_{b, u}: I C 50$ & $0.03(0.03)$ & $0.17(0.06)$ & $0.24(0.08)$ & $0.96(0.39)$ & $1.52(0.17)$ & $1.30(0.29)$ & $1.18(0.04)$ \\
\hline$C_{p}(\mathrm{ng} / \mathrm{ml})$ & $<\operatorname{LLOQ}(\mathrm{NA})$ & $0.04(0.02)$ & $0.07(0.02)$ & $1.13(0.91)$ & $1.98(0.54)$ & $1.51(0.57)$ & $1.08(0.22)$ \\
\hline$C_{p, u}(n M)$ & $N D(N A)$ & $0.01(0.00)$ & $0.01(0.00)$ & $0.22(0.18)$ & $0.38(0.11)$ & $0.29(0.11)$ & $0.21(0.04)$ \\
\hline$C_{p, u}: I C 50$ & $N D(N A)$ & $0.07(0.04)$ & $0.13(0.05)$ & $2.19(1.77)$ & $3.84(1.05)$ & $2.94(1.10)$ & $2.10(0.43)$ \\
\hline$C_{b}: C_{p}$ & $N D(N A)$ & $3.83(0.40)$ & $3.07(0.85)$ & $2.63(0.73)$ & $1.07(0.17)$ & $1.26(0.19)$ & $1.50(0.30)$ \\
\hline$C_{b, u}: C_{p, u}$ & $N D(N A)$ & $1.61(0.17)$ & $1.29(0.36)$ & $1.11(0.31)$ & $0.45(0.07)$ & $0.53(0.08)$ & $0.63(0.13)$ \\
\hline
\end{tabular}

$N A$, not applicable; ND, not determined. Mean $( \pm S E M)$ values of pharmacokinetic parameters $(n=4 /$ time point) of vardenafil at different time points after $1 \mathrm{mg} / \mathrm{kg}$, PO. Samples below the lower limit of quantification ( LLOQ) were set at 0 and included in the calculations. Total plasma $\left(C_{\mathrm{p}}\right)$ or total brain $\left(C_{\mathrm{b}}\right)$ vardenafil concentration is reported as $\mathrm{ng} / \mathrm{ml}$ or $\mathrm{ng} / \mathrm{g}$, respectively. The unbound fractions $\left(F_{u}\right)$ of plasma $(9.5 \%)$ and brain homogenate $(4 \%)$ were determined by equilibrium dialysis experiments performed at Pfizer Inc. Unbound plasma $\left(C_{p, u}\right)$ and unbound brain $\left(C_{b, u}\right)$ concentrations of vardenafil are reported as $n M$ and were calculated as follows; $C_{u}=\left(C_{\text {total }} / \mathrm{MW}\right) \times 1000 \times F_{u}$. Both $C_{\mathrm{b}, u}$ and $C_{\mathrm{p}, \mathrm{u}}$ were compared to the IC50 of vardenafil (0.1 nM, Francis \& Corbin, 2003).

\section{DISCUSSION}

In the reported experiment, we used the ORT with a 10 min retention interval to compare the effects of ICV $(30 \mu \mathrm{g})$ to PO $(1 \mathrm{mg} / \mathrm{kg})$ administration of vardenafil in a cholinergic-deficit model. Both treatments significantly reversed the scopolamineinduced memory deficit when injected within 2 min after learning. Interestingly, ICV administration was also effective 4 min after learning whereas PO administration was not. These findings have two important implications. Firstly, the 2 min difference in effective window could be explained by a difference in time lag before the drug is 
centrally active after ICV and PO administration. On the basis of the behavioral data, this would suggest that orally dosed vardenafil is already centrally available and active within 2-4 min after learning, which is surprisingly fast. These assumptions were confirmed by the pharmacokinetic measurements, showing that vardenafil was $>$ LLOQ plasma and brain within 4 minutes after PO administration. Since we used a 10 min interval, a second implication is that PDE5 inhibition is apparently able to reverse an early consolidation and/or acquisition deficit induced by scopolamine. Thus, PDE5 and CGMP affect acquisition-related processes which appear to take place only a few minutes after the learning trial.

It is known that the muscarinic acetylcholine receptors are crucially important for memory acquisition (Gil-Bea, et al., 2011; Robinson, et al., 2011). A number of studies have reported that an injection of the muscarinic receptor antagonist scopolamine before a learning trial impairs memory, whereas a post-learning blockage of cholinergic receptors is ineffective or actually induces an improvement of memory performance (Blake, Boccia, Krawczyk, \& Baratti, 2011; Rasch, et al., 2006; Young, Bohenek, \& Fanselow, 1995). Therefore, we can assume that scopolamine induces an acquisition, rather than a consolidation deficit. Moreover, we used a 10 min and a $1 \mathrm{~h}$ delay which implies that consolidation processes are not likely to play an important role. Thus, the improved performance in the test trial due to post-learning vardenafil treatment cannot be explained by consolidated long-term memories and related structural changes since these are not expected to occur at these short intervals (Engert \& Bonhoeffer, 1999; Izquierdo, et al., 2006). The mechanism of action behind the attenuation of the cholinergic memory deficit is likely related to the pre-synaptic release of neurotransmitters including glutamate which is mediated by CGMP/PKG signaling and might be enhanced by the inhibition of PDE5 (Arancio, et al., 2001; Hosseini-Sharifabad, et al., 2012; Prickaerts, van Staveren, Sik, Markerink-van Ittersum, Niewöhner, et al., 2002; Puzzo, et al., 2009; Uthayathas, et al., 2013).

The direct ICV administrations can be considered to result in a very fast onset of central action compared to the PO administrations. Interestingly, the time window during which vardenafil attenuated the memory impairment was 2 min longer for the 
ICV administrations than that of the PO administration. Orally dosed vardenafil was only effective when given within 2 min after T1. This implies that vardenafil is centrally active within 2-4 min after oral administration. In accordance with this, our pharmacokinetic measurements showed that vardenafil was >LLOQ in plasma and brain within 4 min after PO administration. Vardenafil was effective when given PO or ICV within 2 and 4 min after $T 1$, respectively. No effects on discrimination performance were observed after vardenafil injections via either route at a later time point. Therefore, it may be assumed that the time window for attenuating the scopolamine-induced deficit extends until 4-6 min after T1.

Since cerebral blood volume accounts for $3-4 \%$ of total brain volume, a drug is considered brain penetrant if its total brain-to-total plasma drug concentration ratio $\left(C_{b}: C_{p}\right)$ is $>0.04$ using non-perfused brain tissue (Hitchcock \& Pennington, 2006). Therefore, the pharmacokinetic data suggest that vardenafil is indeed brain penetrant in rats since its $C_{b}: C_{p}$ is $>1.07$ at all time points (Table 1 ). Most importantly for defining pharmacological exposure-response relationships (Shaffer et al., 2010), the unbound brain-to-unbound plasma drug concentration ratio $\left(C_{b, u}: C_{p, u}\right)$ of vardenafil was $0.45-1.61$ from 2 to 60 min post-dose, suggesting it has net equilibrium at the blood-brain barrier in rats. It is generally accepted that an IC50 is required for a biological effect; the IC50 of vardenafil in vitro is about $0.1 \mathrm{nM}$ (Francis \& Corbin, 2003). Here we show that vardenafil's $C_{b, u}$ reached its IC50 around 10 min after PO administration and remained at or slightly above it for up to $1 \mathrm{~h}$ post-dose (Table 1). In both the $10 \mathrm{~min}$ and $1 \mathrm{~h}$ delay conditions, we observed that PO administered vardenafil improved the memory performance only when injected within 2 min after T1 and not after 4, 6, 15 or 30 min. These observations imply that vardenafil affected acquisition at unbound concentrations below its IC50. On the other hand, when taking only the IC50 as a reference for a relevant biological effect, it could be argued that vardenafil improved retrieval instead of acquisition processes. Along this line of reasoning, the effective PO administrations at 0 and 2 min after T1 would result in $C_{b, u}$ around IC50 during T2, in both the 10 min and $1 \mathrm{~h}$ delay conditions. Yet in the $1 \mathrm{~h}$ delay condition, vardenafil was not effective when injected 4 min after T1, while our pharmacokinetic measurements would predict that the $C_{b, u}$ during $T 2$ would have 
been similar to that of the effective 0 and 2 min injection conditions. Also, despite the observation that vardenafil $C_{b, u}$ would have been well above its IC50 during T2, vardenafil was not effective when injected PO at 15 or 30 min after T1 in the $1 \mathrm{~h}$ delay experiment. Thus, these data do not support a role for improved retrieval processes. Holistically, the pharmacokinetic and behavioral findings suggest that vardenafil reverses the scopolamine-induced deficits in acquisition processes and that these processes can be improved within 4-6 min after the learning trial.

In a prior study by Reneerkens et al. (2012) we reported that $C_{p}$ and $C_{b}$ were considerably higher when compared with the $C_{p}$ and $C_{b}$ levels in the current experiment, $30 \mathrm{~min}$ after administration. When comparing these data directly, about a ten-fold difference in $C_{p}$ levels was observed. Conversely, the $C_{b}$ levels were much more comparable between both studies (when correcting for the dose). Two explanations can be offered for these differences. Firstly, the study by Reneerkens et al. used Wistar Han rats whereas in the present experiment Wistar Harlan rats were used. Differences in metabolism in these strains may explain some differences in $C_{p}$ and $C_{b}$ levels. A second more likely explanation is that these measurements were done in different batches which may also contribute to small differences in these sensitive assays.

In this study we examined the effects of PDE5 inhibition in the scopolamine model, which affects ORT acquisition processes. Combining all findings would suggest a temporal overlap (about 4-6 $\mathrm{min}$ ) between the acquisition and early consolidation phases of memory. This window might represent a transition phase between the acquisition and consolidation stages of memory, as the animals were actually unable to acquire any object information after $\mathrm{T} 1$, and it is unlikely that consolidation processes are already present within 10 min after T1 (Engert \& Bonhoeffer, 1999; Izquierdo, et al., 2006). Therefore, it could be possible that so-called encoding processes are active during this short post-T1 period. Encoding refers to the formation of relations between events in episodic memory, based on many kinds of information, such as temporal and categorical (Lepage, Habib, Cormier, Houle, \& McIntosh, 2000). It also entails novelty/familiarity evaluation (Habib, McIntosh, Wheeler, \& Tulving, 
2003), and involves trans-perceptual processes that operate beyond perceptual processes (Lepage, McIntosh, \& Tulving, 2001). Hence, encoding can continue when the actual stimulus is no longer present. It has been suggested that in humans encoding processes may continue up to 30 min after learning (Rasch, et al., 2006).

In summary, our findings show that oral treatment with vardenafil reversed a scopolamine-induced deficit in object recognition of rats and that this effect was mediated through central acquisition processes. Based on the behavioral and pharmacokinetic data, we estimate that these acquisition processes actually extended for at least 4 min after the learning trial. A major implication of these findings is that drugs that are given immediately after learning to investigate early-consolidation processes might actually also exert their effects in part by influencing acquisition processes. 


\section{REFERENCES}

Akkerman, S., Blokland, A., Reneerkens, O., van Goethem, N. P., Bollen, E., Gijselaers, H. J., et al. (2012). Object recognition testing: Methodological considerations on exploration and discrimination measures. Behav Brain Res, 232(2), 335347.

Arancio, O., Antonova, I., Gambaryan, S., Lohmann, S. M., Wood, J. S., Lawrence, D. S., et al. (2001). Presynaptic role of cGMP-dependent protein kinase during long-lasting potentiation. J Neurosci, 21(1), 143-149.

Arnavaz, A., Aurich, A., Weissenborn, K., Hartmann, U., Emrich, H. M., \& Schneider, U. (2003). Effect of sildenafil (Viagra) on cerebral blood flow velocity: a pilot study. Psychiatry Res, 122(3), 207-209.

Bender, A. T., \& Beavo, J. A. (2006). Cyclic nucleotide phosphodiesterases: molecular regulation to clinical use. Pharmacol Rev, 58(3), 488-520.

Bernabeu, R., Schmitz, P., Faillace, M. P., Izquierdo, I., \& Medina, J. H. (1996). Hippocampal cGMP and CAMP are differentially involved in memory processing of inhibitory avoidance learning. Neuroreport, 7(2), 585-588.

Bernabeu, R., Schroder, N., Quevedo, J., Cammarota, M., Izquierdo, I., \& Medina, J. H. (1997). Further evidence for the involvement of a hippocampal cGMP/cGMPdependent protein kinase cascade in memory consolidation. Neuroreport, 8(9-10), 2221-2224.

Blake, M. G., Boccia, M. M., Krawczyk, M. C., \& Baratti, C. M. (2011). Scopolamine prevents retrograde memory interference between two different learning tasks. Physiol Behav, 102(3-4), 332-337.

Blokland, A., Schreiber, R., \& Prickaerts, J. (2006). Improving memory: a role for phosphodiesterases. Curr Pharm Des, 12(20), 2511-2523.

Devan, B. D., Sierra-Mercado, D., Jr., Jimenez, M., Bowker, J. L., Duffy, K. B., Spangler, E. L., et al. (2004). Phosphodiesterase inhibition by sildenafil citrate attenuates the learning impairment induced by blockade of cholinergic muscarinic receptors in rats. Pharmacol Biochem Behav, 79(4), 691-699.

Engert, F., \& Bonhoeffer, T. (1999). Dendritic spine changes associated with hippocampal long-term synaptic plasticity. Nature, 399(6731), 66-70.

Ennaceur, A., \& Delacour, J. (1988). A new one-trial test for neurobiological studies of memory in rats. 1: Behavioral data. Behav Brain Res, 31(1), 47-59.

Foresta, C., Caretta, N., Zuccarello, D., Poletti, A., Biagioli, A., Caretti, L., et al. (2008). Expression of the PDE5 enzyme on human retinal tissue: new aspects of PDE5 inhibitors ocular side effects. Eye (Lond), 22(1), 144-149.

Francis, S. H., \& Corbin, J. D. (2003). Molecular mechanisms and pharmacokinetics of phosphodiesterase-5 antagonists. Curr Urol Rep, 4(6), 457-465. 
Ghofrani, H. A., \& Grimminger, F. (2006). [Treatment of pulmonary arterial hypertension: phosphodiesterase-5 inhibitors]. Dtsch Med Wochenschr, 131(49 Suppl 9), S311-314.

Ghofrani, H. A., Osterloh, I. H., \& Grimminger, F. (2006). Sildenafil: from angina to erectile dysfunction to pulmonary hypertension and beyond. Nat Rev Drug Discov, 5(8), 689-702.

Gil-Bea, F. J., Solas, M., Mateos, L., Winblad, B., Ramirez, M. J., \& Cedazo-Minguez, A. (2011). Cholinergic hypofunction impairs memory acquisition possibly through hippocampal Arc and BDNF downregulation. Hippocampus, 21(9), 999-1009.

Habib, R., Mclntosh, A. R., Wheeler, M. A., \& Tulving, E. (2003). Memory encoding and hippocampally-based novelty/familiarity discrimination networks. Neuropsychologia, 41(3), 271-279.

Hitchcock, S. A., \& Pennington, L. D. (2006). Structure-brain exposure relationships. J Med Chem, 49(26), 7559-7583.

Hosseini-Sharifabad, A., Ghahremani, M. H., Sabzevari, O., Naghdi, N., Abdollahi, M., Beyer, C., et al. (2012). Effects of protein kinase A and $G$ inhibitors on hippocampal cholinergic markers expressions in rolipram- and sildenafilinduced spatial memory improvement. Pharmacol Biochem Behav, 101(3), 311-319.

Izquierdo, I. n., Bevilaqua, L. R. M., Rossato, J. I., Bonini, J. S., Medina, J. H., \& Cammarota, M. (2006). Different molecular cascades in different sites of the brain control memory consolidation. Trends in Neurosciences, 29(9), 496-505.

Kotera, J., Fujishige, K., \& Omori, K. (2000). Immunohistochemical localization of cGMP-binding cGMP-specific phosphodiesterase (PDE5) in rat tissues. I Histochem Cytochem, 48(5), 685-693.

Lepage, M., Habib, R., Cormier, H., Houle, S., \& McIntosh, A. R. (2000). Neural correlates of semantic associative encoding in episodic memory. Cognitive Brain Research, 9(3), 271-280.

Lepage, M., McIntosh, A. R., \& Tulving, E. (2001). Transperceptual Encoding and Retrieval Processes in Memory: A PET Study of Visual and Haptic Objects. Neurolmage, 14(3), 572-584.

Lin, C. S. (2004). Tissue expression, distribution, and regulation of PDE5. Int J Impot Res, 16 Suppl 1, S8-S10.

Paxinos, G., \& Watson, C. (1996). The rat brain in stereotaxic coordinates (3rd ed.). San Diego: Academic Press.

Prickaerts, J., de Vente, J., Honig, W., Steinbusch, H. W., \& Blokland, A. (2002). CGMP, but not CAMP, in rat hippocampus is involved in early stages of object memory consolidation. Eur J Pharmacol, 436(1-2), 83-87. 
Prickaerts, J., van Staveren, W. C., Sik, A., Markerink-van Ittersum, M., Niewohner, U., van der Staay, F. J., et al. (2002). Effects of two selective phosphodiesterase type 5 inhibitors, sildenafil and vardenafil, on object recognition memory and hippocampal cyclic GMP levels in the rat. Neuroscience, 113, 351-361.

Prickaerts, J., van Staveren, W. C. G., Sik, A., Markerink-van Ittersum, M., Niewöhner, U., van der Staay, F. J., et al. (2002). Effects of two selective phosphodiesterase type 5 inhibitors, sildenafil and vardenafil, on object recognition memory and hippocampal cyclic GMP levels in the rat. Neuroscience, 113(2), 351-361.

Puzzo, D., Sapienza, S., Arancio, O., \& Palmeri, A. (2008). Role of phosphodiesterase 5 in synaptic plasticity and memory. Neuropsychiatr Dis Treat, 4(2), 371-387.

Puzzo, D., Staniszewski, A., Deng, S. X., Privitera, L., Leznik, E., Liu, S., et al. (2009). Phosphodiesterase 5 inhibition improves synaptic function, memory, and amyloid-beta load in an Alzheimer's disease mouse model. J Neurosci, 29(25), 8075-8086.

Rasch, B. H., Born, J., \& Gais, S. (2006). Combined Blockade of Cholinergic Receptors Shifts the Brain from Stimulus Encoding to memory Consolidation Journal of Cognitive Neuroscience, 18(5), 793-802.

Reneerkens, O. A., Rutten, K., Akkerman, S., Blokland, A., Shaffer, C. L., Menniti, F. S., et al. (2012). Phosphodiesterase type 5 (PDE5) inhibition improves object recognition memory: indications for central and peripheral mechanisms. Neurobiol Learn Mem, 97(4), 370-379.

Reneerkens, O. A., Rutten, K., Steinbusch, H. W., Blokland, A., \& Prickaerts, J. (2009). Selective phosphodiesterase inhibitors: a promising target for cognition enhancement. Psychopharmacology (Berl), 202(1-3), 419-443.

Reymann, K. G., \& Frey, J. U. (2007). The late maintenance of hippocampal LTP: requirements, phases, 'synaptic tagging', 'late-associativity' and implications. Neuropharmacology, 52(1), 24-40.

Robinson, L., Platt, B., \& Riedel, G. (2011). Involvement of the cholinergic system in conditioning and perceptual memory. Behav Brain Res, 221(2), 443-465.

Rosengarten, B., Schermuly, R. T., Voswinckel, R., Kohstall, M. G., Olschewski, H., Weissmann, N., et al. (2006). Sildenafil improves dynamic vascular function in the brain: studies in patients with pulmonary hypertension. Cerebrovasc Dis, 21(3), 194-200.

Rutten, K., Prickaerts, J., \& Blokland, A. (2006). Rolipram reverses scopolamineinduced and time-dependent memory deficits in object recognition by different mechanisms of action. Neurobiol Learn Mem, 85(2), 132-138.

Rutten, K., Prickaerts, J., Hendrix, M., van der Staay, F. J., Sik, A., \& Blokland, A. (2007). Time-dependent involvement of cAMP and cGMP in consolidation of object memory: studies using selective phosphodiesterase type 2, 4 and 5 inhibitors. Eur J Pharmacol, 558(1-3), 107-112. 
Rutten, K., Prickaerts, J., Schaenzle, G., Rosenbrock, H., \& Blokland, A. (2008). Subchronic rolipram treatment leads to a persistent improvement in long-term object memory in rats. Neurobiol Learn Mem, 90(3), 569-575.

Rutten, K., Van Donkelaar, E. L., Ferrington, L., Blokland, A., Bollen, E., Steinbusch, H. W., et al. (2009). Phosphodiesterase inhibitors enhance object memory independent of cerebral blood flow and glucose utilization in rats. Neuropsychopharmacology, 34(8), 1914-1925.

Sik, A., van Nieuwehuyzen, P., Prickaerts, J., \& Blokland, A. (2003). Performance of different mouse strains in an object recognition task. Behavioural Brain Research, 147, 49-54.

Stacey, P., Rulten, S., Dapling, A., \& Phillips, S. C. (1998). Molecular cloning and expression of human cGMP-binding CGMP-specific phosphodiesterase (PDE5). Biochem Biophys Res Commun, 247(2), 249-254.

Uthayathas, S., Parameshwaran, K., Karuppagounder, S. S., Ahuja, M., Dhanasekaran, M., \& Suppiramaniam, V. (2013). Selective inhibition of phosphodiesterase 5 enhances glutamatergic synaptic plasticity and memory in mice. Synapse, 67(11), 741-747.

Van Staveren, W. C., Steinbusch, H. W., Markerink-Van Ittersum, M., Repaske, D. R., Goy, M. F., Kotera, J., et al. (2003). mRNA expression patterns of the CGMPhydrolyzing phosphodiesterases types 2, 5, and 9 during development of the rat brain. J Comp Neurol, 467(4), 566-580.

Young, S. L., Bohenek, D. L., \& Fanselow, M. S. (1995). Scopolamine impairs acquisition and facilitates consolidation of fear conditioning: differential effects for tone vs context conditioning. Neurobiol Learn Mem, 63(2), 174180. 

Chapter 5 


\title{
POSSIBLE OVERLAPPING TIME FRAMES OF ACQUISITION AND CONSOLIDATION PHASES IN OBJECT MEMORY PROCESSES: A PHARMACOLOGICAL APPROACH
}

\author{
S. Akkerman, A. Blokland and J. Prickaerts
}




\section{ABSTRACT}

In previous studies, we have shown that acetylcholinesterase inhibitors and phosphodiesterase inhibitors (PDE-Is) are able to improve object memory by enhancing acquisition processes. On the other hand, only PDE-Is improve consolidation processes. Here we show that the cholinesterase inhibitor donepezil also improves memory performance when administered within 2 min after the acquisition trial. Likewise, both PDE5-I and PDE4-I reversed the scopolamine deficit model when administered within 2 min after the learning trial. PDE5-I was effective up to 45 min after the acquisition trial and PDE4-I was effective when administered between $3 \mathrm{~h}$ and $5.5 \mathrm{~h}$ after the acquisition trial. Taken together, our study suggest that acetylcholine, CGMP and CAMP are all involved in acquisition processes and that CGMP and CAMP are also involved in early- and late-consolidation processes, respectively. Most importantly, these pharmacological studies suggest that acquisition processes continue for some time after the learning trial where they share a short common time frame with early consolidation processes. Additional brain concentration measurements of the drugs suggest that these acquisition processes can continue up to 4-6 minutes after learning. 


\section{INTRODUCTION}

Memory is a highly complex cognitive function. The formation of memories requires a sequence of complementary processes to register, maintain and retrieve events. Therefore, it is common to segregate memory processes into an acquisition, consolidation and retrieval phase. When testing cognition enhancing properties of drugs, it is particularly relevant to dissociate its effects on acquisition, consolidation and retrieval, particularly given the accumulating evidence that these different memory processes rely on distinct underlying neurobiological mechanisms (Izquierdo et al., 2006; Winters, Saksida, \& Bussey, 2008). This would not only lead to a better understanding of the properties of a drug, but also of neurobiological mechanisms underlying memory processes.

Memory can be tested in wide array of behavioral animal paradigms. However, few of these models allow for an evaluation of effects on the different memory subprocesses. One trial learning paradigms, such as the passive avoidance test, fear conditioning and the object recognition test (ORT) enable researchers to evaluate memory enhancing effects of drugs on individual memory components. Typically, these tests consist of an acquisition trial in which information is presented, and a test trial in which the information has to be retrieved. In these paradigms, the timing of drug administration determines which memory process is affected (Abel \& Lattal, 2001; van Goethem et al., 2012). That is, when a drug is administered before the acquisition trial it is able to influence acquisition processes, whereas if the drug is given after the acquisition trial it can be assumed to affect consolidation, because acquisition processes are already completed. On the same rationale, drug administration shortly before the test trial will only have an effect on retrieval.

In a previous study, using the object recognition task, we showed that cholinesterase inhibitors (AChE-Is) metrifonate and donepezil enhanced acquisition processes but not consolidation processes, likely to be mediated via an increase in the neurotransmitter acetylcholine (ACh) in the synaptic cleft (Prickaerts et al., 2005). On the other hand, phosphodiesterase type 5 inhibitors (PDE5-Is) affect acquisition and early consolidation 
processes via an increase in intracellular cyclic guanosine monophosphate (cGMP)/Protein kinase G (PKG) signaling, when administered before, or directly after learning, respectively (Bollen et al., 2014; Devan et al., 2007; Devan et al., 2004; Domek-Lopacinska \& Strosznajder, 2008; Prickaerts et al., 2005; van Donkelaar et al., 2008). Also, we found that the phosphodiesterase type 4 inhibitor (PDE4-I) rolipram, which increases cyclic adenosine monophosphate (CAMP)/Protein kinase A (PKA) signaling, improves both acquisition and late consolidation processes when administered before or at $3 \mathrm{~h}$ after learning (Bollen et al., 2014; Rutten, Prickaerts, \& Blokland, 2006; Rutten et al., 2007). These findings provide further support for the notion that acquisition and consolidation processes can be associated with dissociable neurobiological processes.

To more accurately determine the exact time windows of acquisition and consolidation processes occur, we administered donepezil, vardenafil, or rolipram at several specific time points after the acquisition trial. The capability of each drug to improve acquisition and/or consolidation was tested by assessing its potential to prevent natural forgetting in a $24 \mathrm{~h}$ inter-trial interval. Additionally, we tested the effects of the drugs using a $1 \mathrm{~h}$ interval against the muscarinic receptor antagonist scopolamine, which is commonly used as a memory deficit model and specifically blocks acquisition processes (Klinkenberg \& Blokland, 2010; Rutten et al., 2006; Winters, Saksida, \& Bussey, 2006). Furthermore, plasma and brain concentrations of the cognition enhancing drugs were measured at several time points after injection, to find out their relationship with the onset of their behavioral effects.

\section{METHODS}

\section{ANIMALS}

All experimental procedures were approved by the local ethical committee of the Maastricht University for animal experiments according to governmental guidelines. Forty-eight 5-month-old male Wistar rats (Charles River, The Netherlands) were used for the behavioral studies and forty-two of these rats were used for the 
pharmacokinetics study (6 animals were not used). The animals were housed individually in standard Makrolon TM Type III cages on sawdust bedding in an airconditioned room (about $20^{\circ} \mathrm{C}$ ). They were kept under a reversed 12/12-hour light/dark cycle (lights on from 6 PM to 6 AM). Food and water were provided at libitum, water was acidified $(\mathrm{pH} 3)$ to prevent bacterial infections and refreshed every week. Testing was done in the same room as where the animals were housed. A radio played softly, providing background noise $24 \mathrm{~h}$ a day, also during testing.

\section{TREATMENT}

Each compound was freshly dissolved on every experimental day. Donepezil $(1 \mathrm{mg} / \mathrm{kg}$; generously given by Solvay Pharmaceuticals, Weesp, The Netherlands) was dissolved in saline and administered PO, the injection volume was $1 \mathrm{ml} / \mathrm{kg}$. Vardenafil $(1 \mathrm{mg} / \mathrm{kg}$; kindly donated by BAYER AG, Wuppertal, Germany) was dissolved in a vehicle containing $98 \%$ tylose (methyl-cellulose) solution (1\%) and $2 \%$ tween 80 , and was given orally (PO) at an injection volume of $1 \mathrm{ml} / \mathrm{kg}$. The same vehicle was used for dissolving rolipram, which was given $\mathbb{I P}(1 \mathrm{ml} / \mathrm{kg})$. For rolipram (Sigma Aldrich, Zwijndrecht, the Netherlands), a dose of $0.03 \mathrm{mg} / \mathrm{kg}$ was used in the $24 \mathrm{~h}$ retention interval sessions and $0.1 \mathrm{mg} / \mathrm{kg}$ in the $1 \mathrm{~h}$ retention sessions in combination with scopolamine. A fixed dose of $0.1 \mathrm{mg} / \mathrm{kg}$ scopolamine $\mathrm{Hbr}$ (Sigma-Aldrich, Zwijndrecht, the Netherlands) was used as an acquisition deficit model, and was also dissolved in saline. Scopolamine was administered intra peritoneally (IP) at a volume of $1 \mathrm{ml} / \mathrm{kg}$. Doses, vehicles and administration routes were determined based on earlier studies using these drugs (Prickaerts et al., 2005; Rutten et al., 2006; Rutten et al., 2007).

\section{OBJECT RECOGNITION TASK}

We used the object recognition test as described in previous studies (Prickaerts et al., 2002). Briefly, the apparatus consisted of a circular arena, $83 \mathrm{~cm}$ in diameter. Half of the $40 \mathrm{~cm}$ high wall was made of grey polyvinyl chloride (RAL 7035), the other half of transparent polyvinyl chloride. Two objects were placed at symmetrical positions about $10 \mathrm{~cm}$ away from the grey wall. Four different sets of objects were used, and the objects could not be displaced by the animals. 
A test session comprised two trials. During the first trial (T1) the apparatus contained two identical objects (samples). After the first 3 min exploration period the rat was put back in its home cage. Subsequently, after a delay interval of $1 \mathrm{~h}$ or $24 \mathrm{~h}$, the rat was put back in the apparatus for the second trial (T2) that also lasted 3 min. In T2 two dissimilar objects, a familiar one (the sample) and a new one were used. The times spent exploring each object during $\mathrm{T} 1$ and $\mathrm{T} 2$ were recorded manually with specially developed software and stored on a personal computer. The experimenter who scored (live scoring) the behavior was blind towards the treatment conditions of the rats.

Exploration was defined as follows: directing the nose towards the object at a distance of no more than $2 \mathrm{~cm}$ and/or touching the object with the nose. Leaning or climbing on objects was not considered to be exploration. Each object was available in triplicate so none of the two objects from the first trial had to be used as the familiar object in the second trial. In addition, all combinations and locations of objects were used in a balanced manner to reduce potential biases due to preferences for particular locations or objects.

\section{EXPERIMENTAL PROCEDURE AND TREATMENT CONDITIONS}

First, the animals were adapted to the testing procedure and drug administration procedures. During this training phase the rats were submitted to one $3 \mathrm{~min}$ habituation trial in the empty arena on 2 consecutive days. One day later, a $1 \mathrm{~h}$ interval training session (comprising two 3 min trials, i.e., T1 and T2) was performed in which the animals are not subjected to any injections. During this and following sessions two different objects were present. This was followed by a 2nd training session in which a $24 \mathrm{~h}$ interval test was applied, also without injecting the animals. On a subsequent day, animals were familiarized to the administration procedure via saline injections. Animals received two saline injections, one intra peritoneal (IP) injection and one oral (PO; gavage) injection, both $1 \mathrm{ml} / \mathrm{kg}$. Training was completed with a $1 \mathrm{~h}$ interval test, together with an IP injection, 30 min before T1 and a PO injection, directly after $\mathrm{T} 1$. On both occasions saline was administered $(1 \mathrm{ml} / \mathrm{kg})$. After this, drug testing started. 
To specifically investigate the effects of donepezil, vardenafil and rolipram on acquisition processes, a $1 \mathrm{~h}$ delay interval was used in combination with a scopolamine induced memory deficit. One hour intervals were tested 3 times a week, on Monday, Wednesday and Friday, Tuesday and Thursday were used as a washout days.

Since we expected the drug treatments to improve memory consolidation performance we also tested each drug in a $24 \mathrm{~h}$ delay interval. Normally, rats show no more discrimination between the objects after such a delay (Akkerman, et al., 2012). Each week two test sessions were performed, one session on Monday (T1) and Tuesday (T2) and the other one comprised Thursday (T1) and Friday (T2).

\section{TESTING CONDITIONS}

We used 24 rats to examine drug effects in a narrow time window. Donepezil, vardenafil and rolipram were injected at several specific time points, starting directly after T1. At $1 \mathrm{~h}$ delay intervals, these drugs were injected at $0,2,4,15$ or $30 \mathrm{~min}$ after T1, combined with scopolamine treatment at $30 \mathrm{~min}$ before T1. In the control conditions, animals received either scopolamine, or just saline $(1 \mathrm{ml} / \mathrm{kg}, \mathrm{IP}) 30 \mathrm{~min}$ before $\mathrm{T} 1$ in combination with the vehicle of vardenafil and rolipram $(1 \mathrm{ml} / \mathrm{kg}, \mathrm{PO})$ immediately (0 $\mathrm{min}$ ) after $\mathrm{T} 1$.

At $24 \mathrm{~h}$ delay intervals donepezil or vardenafil was injected $0,2,4,15,30,45,60$ or $75 \mathrm{~min}$ after T1. Rolipram was injected at 0, 2, 4, 15 or $30 \mathrm{~min}$ after T1 when tested in the $24 \mathrm{~h}$ delay intervals. As control condition the vehicle of vardenafil and rolipram was injected ( $1 \mathrm{ml} / \mathrm{kg}, \mathrm{PO}$ ) immediately ( $0 \mathrm{~min}$ ) after $\mathrm{T} 1$. The same rats were used for each drug- and interval condition (31 sessions in total). Another batch of 24 rats was used to perform an additional experiment to determine the effective treatment window for rolipram on late consolidation. In this late-consolidation experiment, rolipram was tested when injected at 2.5, 3, 4, 5, 5.5 and $6 \mathrm{~h}$ after T1, each time point had its own vehicle condition. 


\section{PHARMACOKINETICS}

Forty-two rats of the group that were used for behavioral testing were used for the determination of plasma and brain concentrations over the first hour following its oral administration. Animals were sacrificed for blood and brain sampling at 2, 4, 6, 10, 20, 30 and $60 \mathrm{~min}$ after dosing. Twenty-one animals were used for donepezil measurements and 21 animals for rolipram ( $n=3$ per time point). The pharmacokinetics of vardenafil, using similar methods, have been reported elsewhere (Akkerman et al., 2015). Blood was drawn from the saphenus vein using heparincoated tubes, which were temporarily stored on wet ice, and immediately after blood collection the animal was decapitated. The complete brain was collected, rinsed with ice-cold saline, placed in a cup, weighed, and immediately frozen on dry ice. Plasma was isolated using centrifugation ( $1500 \mathrm{G}$ for $10 \mathrm{~min}$ at $4^{\circ} \mathrm{C}$ ) and pipetted into Eppendorf vials. Plasma and brain samples were stored at $-80^{\circ} \mathrm{C}$ until bioanalytical processing. Vardenafil quantification was performed at BioDuro, Pharmaceutical Product Development Inc. (Shanghai, PRC), rolipram and donepezil were quantified by Agilux laboratories (Worcester, USA). For bioanalytical sample preparation, plasma was used as is, while brain samples were first homogenized in a 4-fold volume (w/v) of water (vardenafil) or 80:20 water:acetonitrile (rolipram, donepezil). Both matrices were processed for drug quantification using liquid-liquid extraction methodology followed by a characterized liquid chromatography-tandem mass spectrometry (LCMS/MS) assay. Standard curves were prepared in control matrices, an appropriate dynamic range was achieved, and instrument settings and potentials were adjusted to optimize the MS signal for vardenafil. Vardenafil data were processed using Analyst Software version 1.4.2 (AB Sciex Inc., Ontario, Canada), while for rolipram and donepezil data was processed using Masslynx software with the Quanlynx application manager (Waters Ltd). The Lower Limit Of Quantification (LLOQ) of vardenafil was $0.05 \mathrm{ng} / \mathrm{ml}$ for plasma and $0.02 \mathrm{ng} / \mathrm{ml}$ for brain. For rolipram and donepezil the LLOQ was $0.5 \mathrm{ng} / \mathrm{ml}$ for plasma and $0.25 \mathrm{ng} / \mathrm{ml}$ for brain. The unbound fractions $\left(F_{u}\right)$ of plasma and brain were obtained from the literature and unbound plasma $\left(C_{p / u}\right)$ and unbound brain $\left(C_{b}, u\right)$ concentrations were calculated as follows; $C_{u}=(C$ total / MW) $x$ $1000 \times \mathrm{F}_{\mathrm{u}}$ and reported in $\mathrm{nM}$. Additionally, plasma and brain concentrations were 
compared to IC50 values and the ratio between brain $\left(C_{b}\right)$ and plasma $\left(C_{p}\right)$ concentrations was calculated by dividing $C_{b}$ by $C_{p}$.

\section{STATISTICAL ANALYSIS}

The basic output measures in the ORT are the times spent by rats in exploring each object during T1 and T2. The time spent in exploring the two identical sample objects in T1 will be represented by 'a1' and 'a2'. Time spent in T2 in exploring the sample and the novel object will be represented by 'a3' and 'b', respectively. The following variables were calculated; e1 $=a 1+a 2, e 2=a 3+b$ and $d 2=(b-a 3) / e 2$. e1 and e2 are measures of the total time spent investigating both objects during T1 and T2 respectively. The $d 2$ index is a relative measure of discrimination corrected for the level of exploration in the test-trial (e2).

One-sample t-statistics were performed to assess whether $d 2$ was different from zero, since random exploration in T2 would result in equal exploration of both objects. Between group effects on e1, e2 and the $d 2$ measures were also assessed using oneway ANOVA. In case of significant differences, post-hoc comparisons with the control conditions were performed using Fisher LSD tests.

\section{RESULTS}

\section{EFFECTS OF DONEPEZIL}

The effects of donepezil treatment on the exploration measures are shown in Table 1. In the $1 \mathrm{~h}$ delay interval combined with scopolamine, donepezil and/or scopolamine did not affect the object exploration times $(F(6,49)=1.84$, n.s. $)$ in T1 and T2 $(F(6,49)$ $=1.20$, n.s.). Similarly, after the $24 \mathrm{~h}$ retention delays, no effects of donepezil were found on the exploration levels in $\mathrm{T} 1(F(8,63)=1.27$, n.s. $)$ and $\mathrm{T} 2(F(8,63)=1.11$, n.s.). 
Table 1 Treatment conditions, exploration levels (mean (+SEM)), and number ( $n$ ) of rats in the object recognition task

\begin{tabular}{|c|c|c|c|c|c|c|c|}
\hline & ORT interval & Model & Treatment & Timing & $n$ & e1 & e2 \\
\hline \multirow[t]{13}{*}{ donepezil conditions } & $1 \mathrm{~h}$ & sal & vehicle & $0 \mathrm{~min}$ & 8 & $18.0(1.5)$ & $24.3(2.5)$ \\
\hline & & scop & vehicle & $0 \mathrm{~min}$ & 8 & $26.3(3.3)$ & $27.0(2.4)$ \\
\hline & & scop & $d p z$ & $0 \mathrm{~min}$ & 8 & $27.4(3.8)$ & $26.2(3.7)$ \\
\hline & & scop & $d p z$ & $2 \min$ & 8 & $21.8(2.3)$ & $19.0(2.0)$ \\
\hline & & scop & $d p z$ & $4 \mathrm{~min}$ & 8 & $19.4(1.1)$ & $21.4(2.9)$ \\
\hline & & scop & $d p z$ & $15 \min$ & 8 & $22.4(1.6)$ & $24.3(2.3)$ \\
\hline & & scop & $\mathrm{dpz}$ & $30 \mathrm{~min}$ & 8 & $23.0(2.6)$ & $21.8(2.0)$ \\
\hline & $24 \mathrm{~h}$ & - & vehicle & $0 \mathrm{~min}$ & 8 & $19.8(1.4)$ & $18.2(2.9)$ \\
\hline & & - & $d p z$ & $0 \mathrm{~min}$ & 8 & $21.9(2.0)$ & $18.9(2.1)$ \\
\hline & & - & $\mathrm{dpz}$ & $2 \min$ & 8 & $17.9(1.3)$ & $24.4(1.3)$ \\
\hline & & - & $d p z$ & $4 \mathrm{~min}$ & 8 & $23.7(3.3)$ & $24.2(1.9)$ \\
\hline & & - & $d p z$ & $15 \mathrm{~min}$ & 8 & $19.2(1.8)$ & $19.0(2.3)$ \\
\hline & & - & $\mathrm{dpz}$ & $30 \mathrm{~min}$ & 8 & $23.5(1.5)$ & $19.4(1.9)$ \\
\hline \multirow[t]{9}{*}{ vardenafil conditions } & $24 \mathrm{~h}$ & - & vehicle & $0 \mathrm{~min}$ & 8 & $19.8(1.4)$ & $18.2(2.9)$ \\
\hline & & - & var & $0 \mathrm{~min}$ & 8 & $17.2(1.3)$ & $19.4(1.6)$ \\
\hline & & - & var & $2 \min$ & 8 & $15.4(1.3)$ & $14.3(1.0)$ \\
\hline & & - & var & $4 \min$ & 8 & $15.3(1.6)$ & $17.6(2.0)$ \\
\hline & & - & var & $15 \mathrm{~min}$ & 8 & $19.6(1.5)$ & $21.4(2.3)$ \\
\hline & & - & var & $30 \mathrm{~min}$ & 8 & $15.5(1.3)$ & $18.5(2.1)$ \\
\hline & & - & var & $45 \mathrm{~min}$ & 8 & $20.0(2.8)$ & $24.1(2.2)$ \\
\hline & & - & var & $60 \mathrm{~min}$ & 8 & $20.5(2.0)$ & $22.6(1.3)$ \\
\hline & & - & var & $75 \mathrm{~min}$ & 8 & $20.6(1.8)$ & $23.6(3.5)$ \\
\hline \multirow[t]{25}{*}{ rolipram conditions } & $1 \mathrm{~h}$ & sal & vehicle & $0 \mathrm{~min}$ & 8 & $18.0(1.5)$ & $24.3(2.5)$ \\
\hline & & scop & vehicle & $0 \mathrm{~min}$ & 8 & $26.3(3.3)$ & $27.0(2.4)$ \\
\hline & & scop & roli & $0 \mathrm{~min}$ & 8 & $22.2(2.9)$ & $18.4(1.7)$ \\
\hline & & scop & roli & $2 \min$ & 8 & $23.4(3.5)$ & $17.1(3.2)^{\star}$ \\
\hline & & scop & roli & $4 \mathrm{~min}$ & 8 & $17.1(1.3)$ & $16.4(2.4)^{\star}$ \\
\hline & & scop & roli & $15 \mathrm{~min}$ & 8 & $20.7(2.5)$ & $20.2(1.7)$ \\
\hline & & scop & roli & $30 \mathrm{~min}$ & 8 & $23.4(2.6)$ & $16.5(2.4)^{\star}$ \\
\hline & 24 h early cons. & - & vehicle & $0 \mathrm{~min}$ & 8 & $19.8(1.4)$ & $18.2(2.9)$ \\
\hline & & - & roli & $0 \mathrm{~min}$ & 8 & $17.2(1.7)$ & $17.2(1.2)$ \\
\hline & & - & roli & $2 \min$ & 8 & $14.4(0.8)$ & $18.7(1.1)$ \\
\hline & & - & roli & $4 \mathrm{~min}$ & 8 & $16.3(0.9)$ & $18.8(2.2)$ \\
\hline & & - & roli & $15 \mathrm{~min}$ & 8 & $18.4(1.2)$ & $18.3(1.6)$ \\
\hline & & - & roli & $30 \mathrm{~min}$ & 8 & $17.7(1.6)$ & $17.0(1.5)$ \\
\hline & $24 \mathrm{~h}$ late cons. & - & vehicle & $2.5 \mathrm{~h}$ & 24 & $19.9(1.5)$ & $20.4(1.4)$ \\
\hline & & - & roli & $2.5 \mathrm{~h}$ & 24 & $24.5(1.5)^{*}$ & $27.3(1.7)^{\star *}$ \\
\hline & & - & vehicle & $3 \mathrm{~h}$ & 23 & $21.8(1.5)$ & $24.0(1.5)$ \\
\hline & & - & roli & $3 \mathrm{~h}$ & 24 & $24.1(1.6)$ & $28.7(1.6)^{\star}$ \\
\hline & & - & vehicle & $4 \mathrm{~h}$ & 22 & $19.9(3.6)$ & $22.0(1.9)$ \\
\hline & & - & roli & $4 \mathrm{~h}$ & 22 & $23.1(2.3)$ & $25.0(1.6)$ \\
\hline & & - & vehicle & $5 \mathrm{~h}$ & 20 & $18.4(1.5)$ & $22.9(2.1)$ \\
\hline & & - & roli & $5 \mathrm{~h}$ & 24 & $24.5(2.1)^{*}$ & $27.4(2.0)$ \\
\hline & & - & vehicle & $5.5 \mathrm{~h}$ & 24 & $18.8(1.4)$ & $22.1(1.5)$ \\
\hline & & - & roli & $5.5 \mathrm{~h}$ & 23 & $23.4(1.9)$ & $26.9(1.7)^{*}$ \\
\hline & & - & Vehicle & $6 \mathrm{~h}$ & 21 & $19.7(1.6)$ & $22.1(1.9)$ \\
\hline & & - & roli & $6 \mathrm{~h}$ & 24 & $24.1(1.5)$ & $30.8(1.7)^{\star *}$ \\
\hline
\end{tabular}

Scopolamine (scop) or saline (sal) were always administered 30 min prior to T1. Vehicle, donepezil (dpz), vardenafil (var) and rolipram (roli) were always administered at specific time points after T1, indicated in the Timing column and $\mathrm{e} 1$ and $\mathrm{e} 2$ are the total amounts of exploration during $\mathrm{T} 1$ and $\mathrm{T} 2$, respectively. Significant exploration differences from the vehicle condition are indicated with asterisks, one sample t-test, ${ }^{*}: p<0.05 ;{ }^{* *}: p<0.01$. 
Significant between-group differences on $d 2$ were found in the scopolamine deficit model $(F(8,63)=7.67, p<0.001)$ and natural forgetting paradigm $(F(8,63)=3.49, p$ $<0.01$ ), using one-way ANOVAs. When injected after scopolamine treatment, donepezil attenuated the scopolamine-induced deficit when injected directly up to 2 min after T1, but not when injected after this time point (see Figure 1A). This shows that donepezil treatment improved memory performance after a $24 \mathrm{~h}$ retention delay when injected up to 2 min after T1, but not when injected at 4 min or longer post-T1 intervals (Figure 1B).

A

ORT $1 \mathrm{~h}$ retention interval

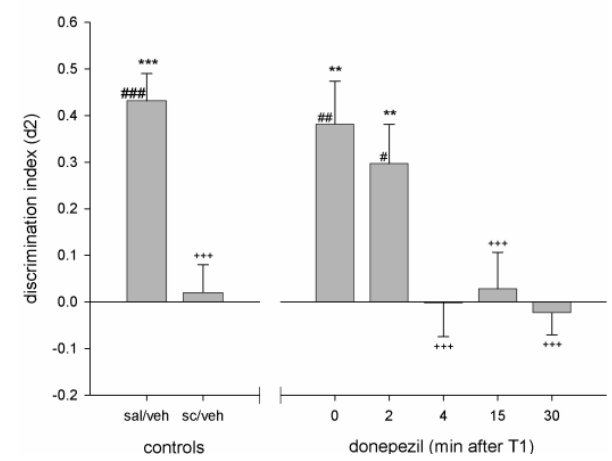

B

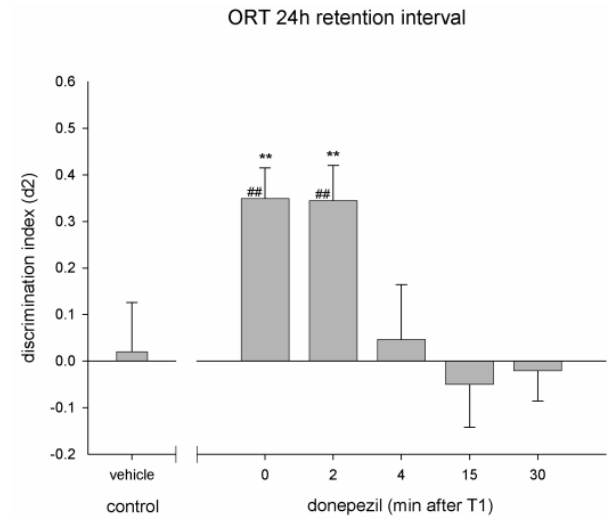

\section{Figure 1 Donepezil}

Effects of donepezil treatment on discrimination performance in an object recognition task using a $1 \mathrm{~h}$ interval in combination with scopolamine $(\mathbf{A})$ or a $24 \mathrm{~h}$ retention interval $(\mathbf{B})$. Asterisks indicate a difference from scopolamine/vehicle (A) or vehicle (B) treated animals, LSD: ${ }^{*}: p<0.01 ; * * *: p<0.001$, plus-signs indicate a difference from the saline/vehicle treated animals (A), LSD, +++: $p<0.001$ and differences from zero are indicated with hashes, one sample $t$-test, \#: $p<0.05 ; \# \#: p<0.01 ; \# \# \#: p<0.001$.

\section{EFFECTS OF VARDENAFIL}

Table 1 shows the effects of vardenafil treatment on the exploration measures. In the $24 \mathrm{~h}$ retention delays, vardenafil did not affect object exploration in $T 1,(F(8,63)=$ 1.83 , n.s.), but exploration differences were found in $T 2,(F(8,63)=2.13, p<0.05)$. However, post-hoc analysis (LSD) showed no significant differences in e2 between the vardenafil conditions and vehicle.

Vardenafil reversed the scopolamine-induced deficits only when injected immediately or 2 min after T1 (see Fig 2A). Differences in $d 2$ measures were found between 
groups in the $24 \mathrm{~h}$ delay interval, $(F(8,63)=2.48, p<0.05)$. Vardenafil treatment improved memory performance in the $24 \mathrm{~h}$ delay interval, when applied between 0 until 45 min after T1, but not when injected at a later time point (Figure 2B).

A

ORT $1 \mathrm{~h}$ retention interval

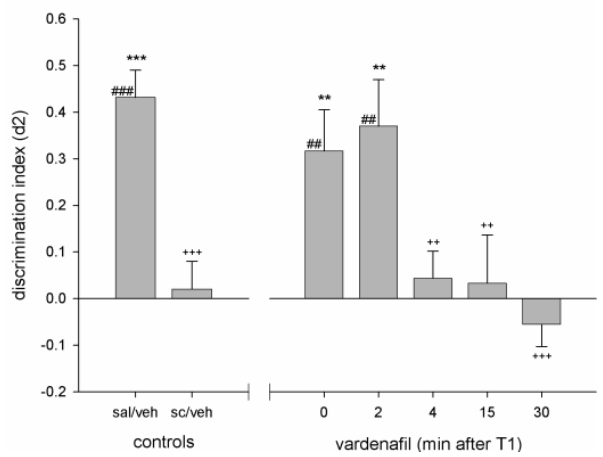

B

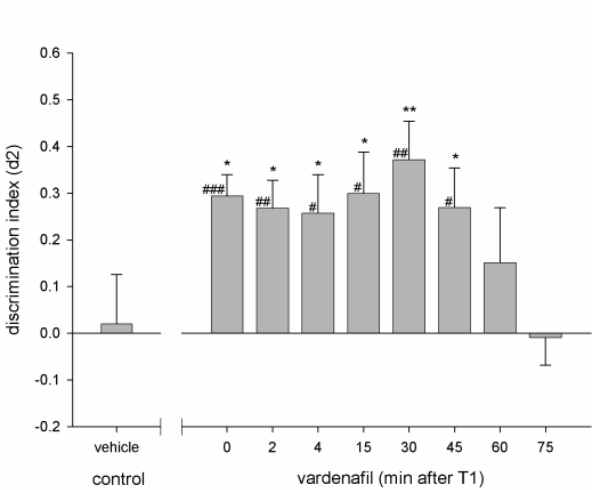

Figure 2 Vardenafil

Effects of vardenafil treatment on discrimination performance in an object recognition task using a $1 \mathrm{~h}$ interval in combination with scopolamine (A): adapted from a previous study (Akkerman et al., 2015) or a $24 \mathrm{~h}$ retention interval (B). Asterisks indicate a difference from scopolamine/vehicle $(\mathbf{A})$ or vehicle $(\mathbf{B})$ treated animals, LSD, $*: p<0.05 ; * *: p<0.01 ; * * *: p<0.001$, plus-signs indicate a difference from the saline/vehicle treated animals (A), LSD, ++: $p<0.01 ;+++: p<0.001$ and differences from zero are indicated with hashes, one sample t-test, \#: $p<0.05$; \#\#: $p<0.01$; \#\#\#: $p<0.001$.

\section{EFFECTS OF ROLIPRAM}

The effects of rolipram treatment on the exploration indices are shown in Table 1. In the $1 \mathrm{~h}$ retention sessions, there were no differences in exploration during $T 1,(F(6,49)$ $=1.51$, n.s.), however, exploration differences were present in $\mathrm{T} 2,(F(6,49)=3.06, p<$ 0.05). Post-hoc analysis (LSD) revealed that all rolipram treated conditions had lower exploration levels in $\mathrm{T} 2$, compared to the vehicle condition. In the $24 \mathrm{~h}$ retention intervals rolipram did not affect object exploration in $\mathrm{T} 1$ and $\mathrm{T} 2,(F(5,42)=2.09$, n.s. $)$ and $(F(5,42)=0.16$, n.s. $)$, respectively.

Significant differences were found in discrimination performance between the treatment conditions of the $1 \mathrm{~h}$ retention experiment, $(F(6,49)=4.06, p<0.01)$. 
A

ORT $1 \mathrm{~h}$ retention interval

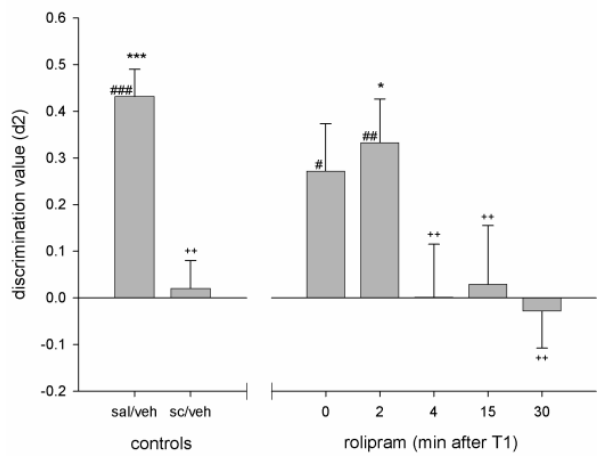

B

ORT 24 retention interval

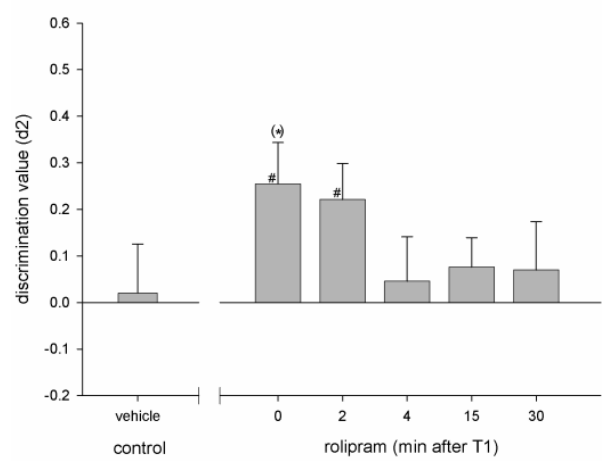

\section{Figure 3 Rolipram early effects}

Effects of rolipram treatment on discrimination performance in an object recognition task using a $1 \mathrm{~h}$ interval in combination with scopolamine $(\mathbf{A})$ or a $24 \mathrm{~h}$ retention interval $(\mathbf{B})$. Asterisks indicate a difference from scopolamine/vehicle $(\mathbf{A})$ or vehicle $(\mathbf{B})$ treated animals, LSD, $\left(^{*}\right) p=0.062 ;{ }^{*}: p<0.05 ;{ }^{* * *}: p<0.001$, plus-signs indicate a difference from the saline/vehicle treated animals (A), LSD, ++: $p<0.01$ and differences from zero are indicated with hashes, one sample t-test, \#: $p<0.05$; \#\#: $p<0.01$; \#\#\#: $p<$ 0.001 .

Post-hoc analysis (LSD) revealed that only animals that received rolipram treatment at 2 min after T1 had significantly higher discrimination compared to vehicle treated animals (Fig 3A). No significant differences were found $(F(5,42)=1.18$, n.s.) between treatment conditions in the $24 \mathrm{~h}$ retention experiment (Fig 3B).

\section{ORT 24 retention interval}

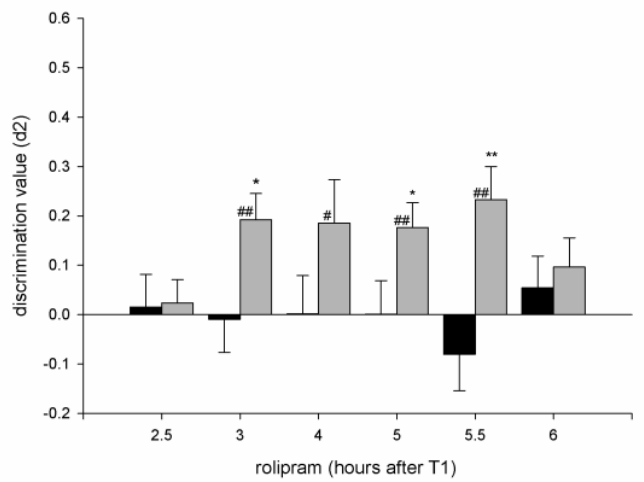

Figure 4 Rolipram late effects

Effects of rolipram treatment on discrimination performance in an object recognition task using a $24 \mathrm{~h}$ retention interval. Asterisks indicate a difference from vehicle treated animals, independent samples $t$-tests; ${ }^{*}: p<0.05 ; * *: p<0.01$ and differences from zero are indicated with hashes, one sample $t$-test, $\#: p<$ $0.05 ; \# \#: p<0.01$. 
In the rolipram late-consolidation experiment, independent samples $t$-tests were performed between the vehicle and rolipram treated animals at each separate injection time point, which showed that animals had object discrimination when treated with rolipram between $3 \mathrm{~h}$ and $5.5 \mathrm{~h}$ after T1 (see Figure 4).

\section{DRUG EXPOSURE-TIME RELATIONSHIPS IN RATS FOLLOWING}

\section{ADMINISTRATION OF VARDENAFIL, ROLIPRAM AND DONEPEZIL}

The results of the pharmacokinetic measurements are shown in Tables 2-3. Donepezil levels >LLOQ in the brain at 4 minutes after PO injection. The unbound brain/plasma ratio varied between 16.66 (10 $\mathrm{min}$ ) to 6.40 (60 $\mathrm{min}$ ) and unbound brain donepezil levels >IC50 were detected 10-60 min after oral administration (see Table 2).

Table 2 Pharmacokinetic measurements of donepezil in the rat after PO administration

\begin{tabular}{|c|c|c|c|c|c|c|c|}
\hline \multirow[b]{2}{*}{ Parameter } & \multicolumn{7}{|c|}{ Time (min) } \\
\hline & 2 & 4 & 6 & 10 & 20 & 30 & 60 \\
\hline $\mathrm{Cb}(\mathrm{ng} / \mathrm{g})$ & $0.00(0.00)$ & $1.70(1.70)$ & $7.29(2.57)$ & $39.73(9.60)$ & $111.65(65.35)$ & $91.80(10.22)$ & $83.97(5.95)$ \\
\hline $\mathrm{Cb}_{\mathrm{b}, u}(\mathrm{nM})$ & $0.00(0.00)$ & $0.79(0.79)$ & $3.36(1.18)$ & $18.32(4.43)$ & $51.49(30.14)$ & $42.33(4.71)$ & $38.72(2.74)$ \\
\hline$C b, u: I C 50$ & $0.00(0.00)$ & $0.14(0.14)$ & $0.59(0.21)$ & $3.21(0.78)$ & $9.03(5.29)$ & $7.43(0.83)$ & $6.79(0.48)$ \\
\hline$C_{p}(n g / m L)$ & $<L L O Q(N A)$ & $<L L O Q(N A)$ & ND & $1.73(1.39)$ & ND & $4.03(0.89)$ & $5.15(0.34)$ \\
\hline$C p, u(n M)$ & $N D(N A)$ & $N D(N A)$ & ND & $2.05(1.64)$ & ND & $4.76(1.05)$ & $6.09(0.40)$ \\
\hline$C p, u: I C 50$ & $N D(N A)$ & $N D(N A)$ & ND & $0.36(0.29)$ & ND & $0.84(0.18)$ & $1.07(0.07)$ \\
\hline $\mathrm{Cb}: \mathrm{Cp}$ & ND (NA) & $N D(N A)$ & ND & $42.75(37.30)$ & ND & $24.28(3.37)$ & $16.41(1.40)$ \\
\hline$C_{b}, u: C_{p, u}$ & $N D(N A)$ & $N D(N A)$ & ND & $16.66(14.54)$ & ND & $9.46(1.31)$ & $6.40(0.55)$ \\
\hline
\end{tabular}

Mean ( \pm SEM) values of pharmacokinetic parameters ( $n=3 /$ time point) of donepezil at different time points after T1, $1 \mathrm{mg} / \mathrm{kg}$, PO Total plasma $\left(C_{p}\right)$ or total brain $\left(C_{b}\right)$ vardenafil concentration is reported as $\mathrm{ng} / \mathrm{mL}$ or $n g / g$, respectively. The unbound fractions $\left(F_{u}\right)$ of plasma $(0.449)$ and brain $(0.175)$ homogenate were obtained from Shen et al. (2011). Unbound plasma $\left(C_{p, u}\right)$ and unbound brain $\left(C_{b, u}\right)$ concentrations of donepezil are reported in $\mathrm{nM}$ and were calculated as follows; $C_{u}=\left(C_{\text {total }} / \mathrm{MW}\right) \times 1000 \times \mathrm{F}_{u}$, with the $\mathrm{MW}$ of donepezil being $379.49 \mathrm{~g} / \mathrm{mol}$. Both $C_{b, u}$ and $C_{p, u}$ were compared to the $I C 50$ of donepezil, $5.7 \mathrm{nM}$ (5.7 nM, Kosasa et al. 2000). ND = not determined, $N A=$ not applicable.

In a previous study we showed that vardenafil levels reached values >LLOQ in both plasma and brain, 4 minutes after PO injection (Akkerman et al., 2015). The reported unbound brain/plasma ratio varied between 1.61 (4 min) to 0.45 (20 $\mathrm{min})$ and unbound brain and plasma levels of vardenafil reached IC50 values around $10 \mathrm{~min}$ after administration and remained >IC50 until 60 min after administration.

Rolipram was given IP and reached $>$ LLOQ levels in the brain at 2 minutes after injection. The unbound brain/plasma ratio varied between 2.50 (4 min) to 6.40 (30 
min) and unbound brain and levels of rolipram reached values >IC50 at 4 min after administration where they remained until 60 min after administration (see Table 3).

Table 3 Pharmacokinetic measurements of rolipram in the rat after IP administration

\begin{tabular}{|c|c|c|c|c|c|c|c|}
\hline \multirow[b]{2}{*}{ Parameter } & \multicolumn{7}{|c|}{ Time (min) } \\
\hline & 2 & 4 & 6 & 10 & 20 & 30 & 60 \\
\hline $\mathrm{Cb}(\mathrm{ng} / \mathrm{g})$ & $3.33(1.77)$ & $16.10(6.44)$ & $29.07(1.99)$ & $23.33(5.95)$ & $14.69(2.42)$ & $8.10(0.69)$ & $4.81(1.20)$ \\
\hline $\mathrm{Cb}, u(\mathrm{nM})$ & $2.32(1.23)$ & $11.22(4.49)$ & $20.27(1.39)$ & $16.27(4.15)$ & $10.24(1.68)$ & $5.65(0.48)$ & $3.36(0.83)$ \\
\hline Cb,u:IC50 & $0.77(0.41)$ & $3.74(1.50)$ & $6.76(0.46)$ & $5.42(1.38)$ & $3.41(0.56)$ & $1.88(0.16)$ & $1.12(0.28)$ \\
\hline$C p(n g / m L)$ & ND & $8.31(5.11)$ & ND & ND & ND & $0.98(0.10)$ & $<L L O Q(N A)$ \\
\hline$C p, u(n M)$ & ND & $7.79(4.79)$ & ND & ND & ND & $0.92(0.10)$ & $N D(N A)$ \\
\hline$C p, u: I C 50$ & ND & $2.60(1.60)$ & ND & ND & ND & $0.31(0.03)$ & $N D(N A)$ \\
\hline $\mathrm{Cb}: \mathrm{Cp}$ & ND & $3.36(1.88)$ & ND & ND & ND & $8.61(1.70)$ & $N D(N A)$ \\
\hline$C_{b}, u: C_{p, u}$ & ND & $2.50(1.40)$ & ND & ND & ND & $6.40(1.26)$ & $N D(N A)$ \\
\hline
\end{tabular}

Mean $( \pm$ SEM) values of pharmacokinetic parameters ( $n=3 /$ time point) of rolipram at different time points after T1, $0.03 \mathrm{mg} / \mathrm{kg}$, IP. Total plasma $\left(C_{p}\right)$ or total brain $\left(C_{b}\right)$ rolipram concentration is reported as $\mathrm{ng} / \mathrm{mL}$ or $n g / g$, respectively. The unbound fractions $\left(F_{u}\right)$ of plasma $(0.258)$ and brain $(0.192)$ homogenate were obtained from Guo et al. (2009). Unbound plasma $\left(C_{p, u}\right)$ and unbound brain $\left(C_{b, u}\right)$ concentrations of rolipram are reported as $n M$ and were calculated as follows: $C_{u}=\left(C_{\text {total }} / M W\right) \times 1000 \times F_{u^{\prime}}$ the $\mathrm{MW}$ of rolipram being $275.34 \mathrm{~g} / \mathrm{mol}$. Both $C_{b, u}$ and $C_{p, u}$ were compared to the $I C 50$ for the rolipram high-affinity-binding site (3 nM) (Barnette et al. 1995; MacKenzie and Houslay 2000). ND = not determined, NA = not applicable.

\section{DISCUSSION}

In this study we examined the effects of different drugs that previously have been characterized as having an effect on acquisition, early-consolidation or lateconsolidation, i.e. donepezil, vardenafil and rolipram, respectively. Here we examined the effects of these drugs in a model of scopolamine induced acquisition impairment and a model of natural forgetting using a $24 \mathrm{~h}$ retention interval. All drugs were already detectable in the brain 2-4 min after injection. Both donepezil and vardenafil reached central levels above IC50 between 10-60 min after injection, while rolipram already reached IC50 within 4 min. In accordance with one of our previous studies (Akkerman et al. 2015) we found that all drugs were able to reverse a scopolamine induced short-term memory deficit when injected within a window of 2 min post-T1. These current findings together with a previous study (Akkerman et al, 2015) then indicate that donepezil, vardenafil and rolipram reverse the scopolamine-induced short-term memory deficit. These effects are assumed to be related to acquisition processes, which appear to linger on for several minutes after the acquisition trial. Donepezil and rolipram also prevented normal forgetting when injected within 2 min after the acquisition trial. These data further suggest an effect on acquisition 
processes. In the $24 \mathrm{~h}$ delay condition the treatment window of vardenafil lasted up to $45 \mathrm{~min}$, indicating that this represents the window for CGMP mediated earlyconsolidation processes. Finally, the data of rolipram in the $24 \mathrm{~h}$ delay interval indicate that CAMP mediated late-consolidation processes take place between 3 and $5.5 \mathrm{~h}$ after acquisition. Taken together, our study suggests that ACh, CGMP and CAMP are all involved in acquisition processes and that CGMP and CAMP are also involved in early- and late-consolidation processes, respectively.

ACh is considered to be an important neurotransmitter for memory function. However, the specific role of ACh in memory formation is still poorly understood, possibly because different stages of memory are differentially affected by it (Micheau \& Marighetto, 2011). The biphasic hypothesis of cholinergic modulation of memory processes states that, ACh enhances the level of afferent input of new information to the cortex via its nicotinic receptors (Hasselmo, 2006). This way, high levels of ACh would lead to efficient acquisition and encoding of new information (Rasch, Born, \& Gais, 2006).

It has been shown that post-learning blockage of muscarinic or nicotinic receptors alone does not impair consolidation processes (Anagnostaras, Maren, Sage, Goodrich, \& Fanselow, 1999; Miranda, Ferreira, Ramírez-Lugo, \& Bermúdez-Rattoni, 2003; Rasch et al., 2006; Rush, 1988; Spangler, Chachich, \& Ingram, 1988). In addition, the retention interval we used was $1 \mathrm{~h}$, which is too short to implicate long-term memory consolidation processes. Therefore, we assume that, in our study the muscarinic antagonist scopolamine induced memory impairment was acquisition related and not due to deficient consolidation.

Surprisingly, the results of the present study showed that, in a $24 \mathrm{~h}$ retention interval, donepezil prevented natural forgetting of object information in rats when administered within 2 min after the T1. However, when injected after 4, 15 or 30 min after $\mathrm{T} 1$, donepezil was no longer effective. Donepezil showed an exactly identical effective window in the scopolamine model. These findings are in accordance with findings from other one-trial recognition studies testing acetylcholinesterase inhibitors (AChE-I) using 24 h delay intervals. Lamirault, Ghuillou, Thal, and Simon (2003) found 
that AChE-I (-)-9-dehydrogalanthaminium bromide successfully improved object and place memory, when injected 20 min before- or directly after the acquisition phase. Also another AChE-I, tacrine, was shown to be effective, when administered directly after learning, in a social recognition task (Gheusi, Bluthe, Goodall, \& Dantzer, 1994). These memory enhancing effects of donepezil on long-term memory are unlikely to be consolidation related. On the contrary, according to the biphasic hypothesis, the increase in cholinergic activity would lead to a reduction of consolidation efficiency (Hasselmo \& Giocomo, 2006; Hasselmo, McGaughy, Laurent Descarries, \& Mircea, 2004; Rasch et al., 2006). Our behavioral findings show that, the window during which cholinergic intervention is able to still influence what is probably acquisition lasts for at least $12 \mathrm{~min}$ (taking into account the injection interval and brain penetration) after a learning trial.

In the $24 \mathrm{~h}$ retention sessions, vardenafil improved object memory when injected up to $45 \mathrm{~min}$ after the learning trial. This finding is in line with our previous studies in which we found an effect when vardenafil was administered immediately after the acquisition trial but not when injected $1 \mathrm{~h}$ after the acquisition trial (Prickaerts et al., 2002; Rutten et al., 2007). The present data provide further support that enhancing effects of vardenafil on memory consolidation processes can be found up to $50 \mathrm{~min}$ after T1 (taking into account the injection interval and brain penetration). The effect of vardenafil on consolidation processes is likely to be mediated via glutamatergic stimulation of post-synaptic GMP/PKG signaling (Bollen et al., 2014; Lu, Kandel, \& Hawkins, 1999).

In contrast to the current $24 \mathrm{~h}$ retention experiment, vardenafil could only reverse a scopolamine-induced acquisition deficit when injected within 2 min after the learning trial (see also Akkerman et al., 2015). This effective window shows surprising similarities to our current findings with donepezil and may suggest that the effect of vardenafil on long-term memory is partly mediated through acquisition processes when treatment is performed shortly after the acquisition trial. Such an effect on acquisition might be explained by an increased release of neurotransmitters, including 
ACh and glutamate, as a results of presynaptic stimulation of cGMP/PKG signaling by vardenafil (Arancio, Kandel, \& Hawkins, 1995).

Surprisingly, the results obtained with rolipram treatment within the first $\mathrm{h}$ after $\mathrm{T1}$ were very similar to those found with donepezil, however, the effects were a bit less pronounced. Rolipram only fully reversed the scopolamine-induced deficit when injected at 2 min after T1, but an intermediate effect was found when rolipram was injected immediately after $\mathrm{T} 1$, that is, the $d 2$ index of rats injected at 0 min after $\mathrm{T} 1$ was higher compared to zero but not compared to vehicle treated animals after $24 \mathrm{~h}$. Also in the $24 \mathrm{~h}$ retention interval, an intermediate effect of rolipram treatment was found within the first 2 min after T1. In previous studies we never found that rolipram was effective when injected directly after T1 using a $24 \mathrm{~h}$ delay interval (Bollen et al., 2014; Rutten et al., 2006; Rutten et al., 2007; Rutten et al., 2009). However, in those studies our injection times were on average about 4-10 min after the acquisition trial due to the injection procedures including taking the rat from the apparatus and taking the injection needle(s) (see Bollen et al., 2014).

Importantly, in the present study we took great effort and care to inject exactly at the intended time point after T1. This might explain the lack of previous findings, as the current effects of rolipram were very subtle. Rolipram increases levels of CAMP (Barad, Bourtchouladze, Winder, Golan, \& Kandel, 1998; Rutten et al., 2006), and presynaptical elevation of CAMP might induce the release of neurotransmitters, including ACh (Schoffelmeer, et al., 1985). Along similar lines, it has been shown that acute administration of a CAMP analog, directly after learning can improve long-term habituation in crabs (Romano, Locatelli, Delorenzi, Pedreira, \& Maldonado, 1996). This effect might be related a short initial spike in cAMP levels, immediately after learning (Izquierdo et al., 2006). In our study, a reinforcement of this initial spike, by rolipram treatment, may just have been sufficient to enhance the memory trace via acquisition processes.

In agreement with our previous studies (Akkerman, Blokland, \& Prickaerts, 2014; Rutten et al., 2007) we observed that rolipram improves late consolidation processes when injected at $3 \mathrm{~h}$ after the acquisition trial. We now demonstrated that rolipram is 
exactly effective when given between 3 and $5.5 \mathrm{~h}$ after the acquisition trial. This late consolidation window is likely to be dependent on postsynaptic CAMP/PKA signaling (Barad et al., 1998; Rutten et al., 2006; Bollen et al. 2014) and therefore indicates that cAMP mediated late-consolidation processes take place at this time.

Remarkably, in the scopolamine deficit model all drugs were effective when injected within 2 min after T1. However, to determine the exact duration of the target processes one has to know the pharmacokinetics of each drug, because the drugs needs some time to reach the brain instantly after peripheral administration. From the literature it is known that donepezil reaches maximum plasma levels 13 min after IP injection at a dose of $1 \mathrm{mg} / \mathrm{kg}$ (Nakashima, Itoh, Kono, Nakashima, \& Wada, 2006). Maximum plasma and brain concentrations are reached simultaneously at approximately $30 \mathrm{~min}$ after PO donepezil administration (1 mg/kg) with a half-life of about 1 h (Kenji Matsui, Mannen Mishima, Yasushi Nagai, Teruaki Yuzuriha, \& Tsutomu Yoshimura, 1999). Thus, it seems that drug absorption following IP administration is about twice as rapid as PO administration, although the observed difference in the onset of behavioral effects is smaller (Snape et al., 1999).

Our PK measurements appear to corroborate with these reports, although we already observed maximum concentrations in brain and plasma at 20 min post administration the concentrations at 30 min were almost identical to those observed by Matsui et al. (1999). It is unknown what donepezil concentration is required in the brain for memory enhancement, though it is generally accepted that an IC50 is required for a biological effect. Here we show that donepezil's $C_{b, u}$ reached its IC50 within 10 min after PO administration and remained at or above it for up to $1 \mathrm{~h}$ post-dosing (Table 2). The latter is an important observation since it explains that the effect of donepezil given at 0 and 2 min after the acquisition trial cannot be explained via an effect on retrieval, as one would expect that the administration at 4, 15 or 30 min after T1 in the $1 \mathrm{~h}$ interval should also be effective if retrieval was involved, i.e. all these administrations would result in brain concentrations well above IC50 at the time of retrieval (T2). Also, based on the available PK, one can assume that donepezil brain concentrations after the $24 \mathrm{~h}$ delay interval were too low for an effect on retrieval. 
Additionally, the $1 \mathrm{~h}$ interval is too short to implicate late consolidation processes which are linked to long-term memory. Taken together these findings indicate acquisition processes as the most likely mechanism for the observed discrimination improvement.

Donepezil always improved memory when injected within 2 min after learning. Together with the IC 50 this also suggests that donepezil, i.e. ACh, is active within the first $12 \mathrm{~min}$ after T1. Yet it cannot be ruled out that donepezil already affects acquisition at unbound concentrations below its IC 50, i.e. directly after the drug becomes centrally available. As we first detected donepezil in the brain 4 minutes after PO administration, the window might actually persist for only 6 min after T1.

Vardenafil was detectable in the brain within $2 \mathrm{~min}$ and reaches IC 50 values at $10 \mathrm{~min}$ after PO injection (see Table 3). Also based on PK measurements and behavioral data in our previous study (Akkerman et al., 2015), we argue that vardenafil is likely to be involved in acquisition processes and that these processes could extend at least 4-6 min after T1. This acquisition window matches closely with the 6 min window found for donepezil. In the current study we further show that, when memory is not impaired by scopolamine, vardenafil prevented natural forgetting when injected up to 45 min after T1, implying that the CGMP/PKG pathway is involved in both acquisition and early consolidation processes.

Rolipram has been reported to reach maximum plasma levels 15 min after oral administration (Krause \& Kuhne, 1988). In our study rolipram was administered IP, and is therefore likely to be more rapidly absorbed into the bloodstream. Our PK measurements showed that rolipram is present in the brain at 2 min after injection and unbound rolipram reached the IC 50 concentration in the brain within 4 min after injection. The maximum concentration was reached at 6 min and returned to IC 50 at $1 \mathrm{~h}$ after injection. Combined with the behavioral data it can be argued that rolipram may be actually active up to 4-6 min after learning. Considering our finding in the scopolamine model, it is unlikely that retrieval processes were affected by rolipram because, like with donepezil, every administration time point resulted in a brain concentration above IC50 at the time of retrieval (T2). Furthermore, the half-life of 
rolipram is too short for any effective concentrations at the time of retrieval after the $24 \mathrm{~h}$ retetention interval. As the $1 \mathrm{~h}$ interval is too short to accommodate consolidation processes, these findings implicate that PDE4 is involved in acquisition processes and again, these seem to extend for 4-6 min after T1.

Our findings on the windows of effect of donepezil, vardenafil and rolipram suggest a temporal overlap, of at least 4-6 minutes, between the acquisition and early consolidation phases of memory. This window might represent a transition between acquisition processes and the consolidation stage of memory. The animals were unable to acquire object information after T1, since they have no access to the objects anymore. Also, as described above, it is unlikely that donepezil enhances consolidation. Therefore, it is possible that there are encoding processes that continue for some time after stimulus exposure. Encoding refers to the formation of relations between events in episodic memory, based on many kinds of information, such as temporal and categorical (Lepage, Habib, Cormier, Houle, \& McIntosh, 2000). It also entails novelty/familiarity evaluation (Habib, Mclntosh, Wheeler, \& Tulving, 2003), and involves trans-perceptual processes operate beyond perceptual processes (Lepage, Mclntosh, \& Tulving, 2001).

In our experiment all drugs had a remarkably similar effective window of 2 min postT1, during which they were capable to restore the scopolamine memory deficit. Combined with our PK measurements these data suggest that acquisition processes continue for 4-6 min after T1. Vardenafil prevented natural forgetting via CGMP/PKG early consolidation processes when injected within 0-45 min after T1. This shows that there is a 4-6 min window of overlap between acquisition and consolidation processes, during which the transition from acquisition into early-consolidation processes might take place. Furthermore, our data indicates that CAMP/PKA signaling is crucially involved in late consolidation processes between 3 and $5.5 \mathrm{~h}$ after T1. It would be interesting to identify pathways that are active in-between $45 \mathrm{~min}$ and $3 \mathrm{~h}$ after T1, which might link these CGMP and CAMP signaling pathways and investigate these in a similar fashion. Knowing the complete cascades underlying long-term memory formation may give us new insight and strategies to effectively treat cognitive decline. 


\section{REFERENCES}

Abel, T., \& Lattal, K.M. (2001). Molecular mechanisms of memory acquisition, consolidation and retrieval. Current Opinion in Neurobiology, 11(2), 180-187.

Akkerman, S., Blokland, A., \& Prickaerts, J. (2014). Mind the gap: Delayed manifestation of long-term object memory improvement by phosphodiesterase inhibitors. Neurobiol Learn Mem, 109, 139-143.

Akkerman, S., Blokland, A., van Goethem, N.P., Cremers, P., Shaffer, C.L., Osgood, S.M., Steinbusch, H. \& Prickaerts, J. (2015). PDE5 inhibition improves acquisition processes after learning via a central mechanism. Neuropharmacology, 97, 233-239.

Akkerman, S., Blokland, A., Reneerkens, O., van Goethem, N.P., Bollen, E., Gijselaers, H.J. (2012). Object recognition testing: Methodological considerations on exploration and discrimination measures. Behav Brain Res, 232(2), 335-347.

Anagnostaras, S.G., Maren, S., Sage, J.R., Goodrich, S., \& Fanselow, M.S. (1999). Scopolamine and Pavlovian Fear Conditioning in Rats: Dose-Effect Analysis. Neuropsychopharmacology, 21(6), 731-744.

Arancio, O., Kandel, E.R., \& Hawkins, R.D. (1995). Activity-dependent long-term enhancement of transmitter release by presynaptic $3^{1}, 5^{\prime}$-cyclic GMP in cultured hippocampal neurons. Nature, 376(6535), 74-80.

Barad, M., Bourtchouladze, R., Winder, D.G., Golan, H., \& Kandel, E. (1998). Rolipram, a type IV-specific phosphodiesterase inhibitor, facilitates the establishment of long-lasting long-term potentiation and improves memory. Proc Natl Acad Sci U S A, 95(25), 15020-15025.

Barnette, M.S., Grous, M., Cieslinski, L.B., Burman, M., Christensen, S.B., \& Torphy, T.J. (1995). Inhibitors of phosphodiesterase IV (PDE IV) increase acid secretion in rabbit isolated gastric glands: correlation between function and interaction with a high-affinity rolipram binding site. J Pharmacol Exp Ther, 273(3), 13961402.

Bollen, E., Puzzo, D., Rutten, K., Privitera, L., De Vry, J., Vanmierlo, T. (2014). Improved Long-Term Memory via Enhancing CGMP-PKG Signaling Requires CAMP-PKA Signaling. Neuropsychopharmacology, 39(11), 2497-505.

Devan, B.D., Pistell, P.J., Daffin, J.L.W., Nelson, C.M., Duffy, K.B., Bowker, J.L. (2007). Sildenafil citrate attenuates a complex maze impairment induced by intracerebroventricular infusion of the NOS inhibitor N[omega]-nitro-Larginine methyl ester. European Journal of Pharmacology, 563(1-3), 134-140.

Devan, B.D., Sierra-Mercado, J.D., Jimenez, M., Bowker, J.L., Duffy, K.B., Spangler, E.L. (2004). Phosphodiesterase inhibition by sildenafil citrate attenuates the learning impairment induced by blockade of cholinergic muscarinic receptors in rats. Pharmacology Biochemistry and Behavior, 79(4), 691-699. 
Domek-Lopacinska, K., \& Strosznajder, J.B. (2008). The effect of selective inhibition of cyclic GMP hydrolyzing phosphodiesterases 2 and 5 on learning and memory processes and nitric oxide synthase activity in brain during aging. Brain Research, 1216, 68-77.

Francis, S.H., \& Corbin, J.D. (2003). Molecular mechanisms and pharmacokinetics of phosphodiesterase-5 antagonists. Curr Urol Rep, 4(6), 457-465.

Gheusi, G., Bluthe, R.M., Goodall, G., \& Dantzer, R. (1994). Ethological study of the effects of tetrahydroaminoacridine (THA) on social recognition in rats. Psychopharmacology (Berl), 114(4), 644-650.

Guo, Q., Brady, M., \& Gunn, R.N. (2009). A biomathematical modeling approach to central nervous system radioligand discovery and development. I Nucl Med, 50(10), 1715-1723.

Habib, R., McIntosh, A.R., Wheeler, M.A., \& Tulving, E. (2003). Memory encoding and hippocampally-based novelty/familiarity discrimination networks. Neuropsychologia, 41(3), 271-279.

Hasselmo, M.E. (2006). The role of acetylcholine in learning and memory. Curr Opin Neurobiol, 16(6), 710-715.

Hasselmo, M.E., \& Giocomo, L.M. (2006). Cholinergic modulation of cortical function. J Mol Neurosci, 30(1-2), 133-135.

Hasselmo, M.E., McGaughy, J., Laurent Descarries, K.K., \& Mircea, S. (2004). High acetylcholine levels set circuit dynamics for attention and encoding and low acetylcholine levels set dynamics for consolidation Progress in Brain Research (Vol. Volume 145, pp. 207-231): Elsevier.

Izquierdo, I., Bevilaqua, L.R., Rossato, J.I., Bonini, J.S., Medina, J.H., \& Cammarota, M. (2006). Different molecular cascades in different sites of the brain control memory consolidation. Trends Neurosci, 29(9), 496-505.

Klinkenberg, I., \& Blokland, A. (2010). The validity of scopolamine as a pharmacological model for cognitive impairment: a review of animal behavioral studies. Neurosci Biobehav Rev, 34(8), 1307-1350.

Kosasa, T., Kuriya, Y., Matsui, K., \& Yamanishi, Y. (2000). Inhibitory effect of orally administered donepezil hydrochloride (E2020), a novel treatment for Alzheimer's disease, on cholinesterase activity in rats. Eur J Pharmacol, 389(23), 173-179.

Krause, W., \& Kuhne, G. (1988). Pharmacokinetics of rolipram in the rhesus and cynomolgus monkeys, the rat and the rabbit. Studies on species differences. Xenobiotica, 18, 561-571.

Lamirault, L., Guillou, C., Thal, C., \& Simon, H. (2003). (-)-9-Dehydrogalanthaminium bromide, a new cholinesterase inhibitor, enhances place and object recognition memory in young and old rats. Neurobiol Learn Mem, 80(2), 113122. 
Lepage, M., Habib, R., Cormier, H., Houle, S., \& Mclntosh, A.R. (2000). Neural correlates of semantic associative encoding in episodic memory. Cognitive Brain Research, 9(3), 271-280.

Lepage, M., Mclntosh, A.R., \& Tulving, E. (2001). Transperceptual Encoding and Retrieval Processes in Memory: A PET Study of Visual and Haptic Objects. Neurolmage, 14(3), 572-584.

Lu, Y.F., Kandel, E.R., \& Hawkins, R.D. (1999). Nitric oxide signaling contributes to late-phase LTP and CREB phosphorylation in the hippocampus. I Neurosci, 19(23), 10250-10261.

MacKenzie, S.J., \& Houslay, M.D. (2000). Action of rolipram on specific PDE4 CAMP phosphodiesterase isoforms and on the phosphorylation of CAMP-responseelement-binding protein (CREB) and p38 mitogen-activated protein (MAP) kinase in U937 monocytic cells. Biochem J, 347(Pt 2), 571-578.

Matsui, K., Mishima, M., Nagai, Y., Yuzuriha, T., \& Yoshimura, T. (1999). Absorption, distribution, metabolism, and excretion of donepezil (Aricept) after a single oral administration to Rat. Drug Metab Dispos, 27(12), 1406-1414.

Matsui, K., Mishima, M., Nagai, Y., Yuzuriha, T., \& Yoshimura, T. (1999). Absorption, Distribution, Metabolism, and Excretion of Donepezil (Aricept) after a Single Oral Administration to Rat. Drug Metabolism and Disposition, 27(12), 14061414.

Micheau, J., \& Marighetto, A. (2011). Acetylcholine and memory: A long, complex and chaotic but still living relationship. Behav Brain Res, 221(2), 424-429.

Miranda, M.I., Ferreira, G., Ramírez-Lugo, L., \& Bermúdez-Rattoni, F. (2003). Role of cholinergic system on the construction of memories: Taste memory encoding. Neurobiology of Learning and Memory, 80(3), 211-222.

Nakashima, K., Itoh, K., Kono, M., Nakashima, M.N., \& Wada, M. (2006). Determination of donepezil hydrochloride in human and rat plasma, blood and brain microdialysates by HPLC with a short C30 column. Journal of Pharmaceutical and Biomedical Analysis, 41(1), 201-206.

Prickaerts, J., Sik, A., van der Staay, F.J., de Vente, J., \& Blokland, A. (2005). Dissociable effects of acetylcholinesterase inhibitors and phosphodiesterase type 5 inhibitors on object recognition memory: acquisition versus consolidation. Psychopharmacology (Berl), 177(4), 381-390.

Prickaerts, J., van Staveren, W.C.G., Sik, A., Markerink-van Ittersum, M., Niewöhner, U., van der Staay, F.J. (2002). Effects of two selective phosphodiesterase type 5 inhibitors, sildenafil and vardenafil, on object recognition memory and hippocampal cyclic GMP levels in the rat. Neuroscience, 113(2), 351-361.

Rasch, B.H., Born, J., \& Gais, S. (2006). Combined Blockade of Cholinergic Receptors Shifts the Brain from Stimulus Encoding to memory Consolidation Journal of Cognitive Neuroscience, 18(5), 793-802. 
Romano, A., Locatelli, F., Delorenzi, A., Pedreira, M.E., \& Maldonado, H. (1996). Effects of activation and inhibition of CAMP-dependent protein kinase on long-term habituation in the crabChasmagnathus. Brain Research, 735(1), 131-140.

Rush, D.K. (1988). Scopolamine amnesia of passive avoidance: A deficit of information acquisition. Behavioral and Neural Biology, 50(3), 255-274.

Rutten, K., Prickaerts, J., \& Blokland, A. (2006). Rolipram reverses scopolamineinduced and time-dependent memory deficits in object recognition by different mechanisms of action. Neurobiol Learn Mem, 85(2), 132-138.

Rutten, K., Prickaerts, J., Hendrix, M., van der Staay, F.J., Sik, A., \& Blokland, A. (2007). Time-dependent involvement of cAMP and cGMP in consolidation of object memory: studies using selective phosphodiesterase type 2, 4 and 5 inhibitors. Eur J Pharmacol, 558(1-3), 107-112.

Rutten, K., Prickaerts, J., Schaenzle, G., Rosenbrock, H., \& Blokland, A. (2008). Subchronic rolipram treatment leads to a persistent improvement in long-term object memory in rats. Neurobiol Learn Mem, 90(3), 569-575.

Rutten, K., Van Donkelaar, E.L., Ferrington, L., Blokland, A., Bollen, E., Steinbusch, H.W. (2009). Phosphodiesterase inhibitors enhance object memory independent of cerebral blood flow and glucose utilization in rats. Neuropsychopharmacology, 34(8), 1914-1925.

Schoffelmeer, A.N., Wardeh, G., Mulder, A.H. (1986). Cyclic AMP facilitates the electrically evoked release of radiolabelled noradrenaline, dopamine and 5hydroxytryptamine from rat brain slices. Naunyn Schmiedebergs Arch Pharmacol. 330(1), 74-76.

Shen, F., Smith, J.A., Chang, R., Bourdet, D.L., Tsuruda, P.R., Obedencio, G.P. (2011). 5-HT(4) receptor agonist mediated enhancement of cognitive function in vivo and amyloid precursor protein processing in vitro: A pharmacodynamic and pharmacokinetic assessment. Neuropharmacology, 61(1-2), 69-79.

Snape, M.F., Misra, A., Murray, T.K., De Souza, R.J., Williams, J.L., Cross, A.J. (1999). A comparative study in rats of the in vitro and in vivo pharmacology of the acetylcholinesterase inhibitors tacrine, donepezil and NXX-066. Neuropharmacology, 38(1), 181-193.

Spangler, E.L., Chachich, M.E., \& Ingram, D.K. (1988). Scopolamine in rats impairs acquisition but not retention in a 14-unit T-maze. Pharmacology Biochemistry and Behavior, 30(4), 949-955.

van Donkelaar, E.L., Rutten, K., Blokland, A., Akkerman, S., Steinbusch, H.W.M., \& Prickaerts, J. (2008). Phosphodiesterase 2 and 5 inhibition attenuates the object memory deficit induced by acute tryptophan depletion. European Journal of Pharmacology, 600(1-3), 98-104.

van Goethem, N.P., Rutten, K., van der Staay, F.J., Jans, L.A.W., Akkerman, S., Steinbusch, H.W.M. (2012). Object recognition testing: Rodent species, 
strains, housing conditions, and estrous cycle. Behavioural Brain Research, 232(2), 323-334.

Winters, B.D., Saksida, L.M., \& Bussey, T.J. (2006). Paradoxical facilitation of object recognition memory after infusion of scopolamine into perirhinal cortex: implications for cholinergic system function. J Neurosci, 26(37), 9520-9529. doi: 26/37/9520

Winters, B.D., Saksida, L.M., \& Bussey, T.J. (2008). Object recognition memory: Neurobiological mechanisms of encoding, consolidation and retrieval. Neuroscience \& Biobehavioral Reviews, 32(5), 1055-1070. 
Ch apter $5 \mid 141$ 
Chapter 6 


\section{MIND THE GAP: DELAYED}

MANIFESTATION OF LONG-TERM OBJECT MEMORY IMPROVEMENT BY PHOSPHODIESTERASE INHIBITORS

S. Akkerman, A. Blokland and J. Prickaerts 


\section{ABSTRACT}

We examined the temporal profile of pharmacologically enhanced episodic memory, using the object recognition task. Male Wistar rats were tested at different retention intervals ranging from $1 \mathrm{~h}-24 \mathrm{~h}$. The object discrimination performance of all groups (untreated, placebo, drug treatment) gradually decreased up to an interval ( $8 \mathrm{~h}$ ). Interestingly, only after this $8 \mathrm{~h}$ interval the memory improving effects of vardenafil and rolipram started to emerge. This time-dependent memory performance shows similarities with the Kamin effect. The delayed manifestation of drug-enhanced memory suggests that two separate memory mechanisms are at play, a quick transient form of memory and a more stable memory form that requires several hours to develop. It is important to take this into account when testing treatments intended for long-term memory enhancement. 


\section{INTRODUCTION}

The object recognition tasks (ORT) is a widely used one-trial memory task, allowing the assessment of the effects of interventions on the different stages of memory (Abel \& Lattal, 2001; Prickaerts, Sik, van der Staay, de Vente, \& Blokland, 2005; Ennaceur, 2010). We have shown that, under our standard test conditions, rats have good object memory after a $1 \mathrm{~h}$ inter-trial delay and that the rats do not discriminate between the familiar and novel object after 24 h, i.e. no memory (Rutten, Prickaerts, \& Blokland, 2006; Akkerman et al., 2012a). This time-dependent forgetting after $24 \mathrm{~h}$ can be prevented by treatment with selective phosphodiesterase (PDE) inhibitors which inhibit the degradation of the second messengers CGMP and/or CAMP. The CGMP-specific PDE5 inhibitors (PDE5-I) are effective when injected shortly before learning or immediately after learning. CAMP-specific PDE4 inhibitors (PDE4-I) also improve memory performance but only when given $3 \mathrm{~h}$ after learning (Bernabeu et al., 1997; Prickaerts, de Vente, Honig, Steinbusch, \& Blokland, 2002; Devan et al., 2004; Rutten et al., 2006; Rutten et al., 2007; Levallet, Hotte, Boulouard, \& Dauphin, 2009; Bruno et al., 2011; Reneerkens et al., 2012). It has been proposed that PDE5 and PDE4 inhibition lead to improvement of early- and late consolidation processes, respectively. However, it has not yet been investigated how memory enhanced by PDE inhibition is expressed at intermediate intervals between $1 \mathrm{~h}$ and $24 \mathrm{~h}$.

To evaluate the stability of the memory trace during the different stages of memory consolidation we tested rats in the ORT on 6 different retention intervals; 1 h, 4 h, 8 $h, 10 \mathrm{~h}, 12 \mathrm{~h}$ and $24 \mathrm{~h}$. First, untreated and vehicle treated animals were used to investigate the temporal profile of normal forgetting in the ORT. Subsequently, the effects of vardenafil (PDE5-I) or rolipram (PDE4-I) were tested at the different inter-trial intervals. 


\section{Materials ANd Methods}

\section{ANIMALS}

Two cohorts of twenty-four 3-months-old male Wistar rats (Charles River, Sulzfeld, Germany) were used. The animals were housed individually in standard Makrolon TM Type III cages on sawdust bedding in an air-conditioned room (about $20^{\circ} \mathrm{C}$ ). They were kept under a reversed 11/13-hour light/dark cycle (lights on from 6 PM to 7 AM). Food and water were provided ad libitum. Animals were housed and tested in the same room while a radio provided background noise $24 \mathrm{~h}$ a day.

\section{DRUGS}

Four different treatments were tested, vehicle, rolipram, vardenafil and no treatment. Test compounds were freshly dissolved on every experimental day. Vehicle composition was similar for rolipram and vardenafil and consisted of a $1 \%$ methylcellulose solution and tween 80 , proportions were $98 \%$ and $2 \%$ respectively. Rolipram $(0.03 \mathrm{mg} / \mathrm{kg}$ in $1 \mathrm{ml} / \mathrm{kg}$ ) was administered intra peritoneally (IP), $3 \mathrm{~h}$ after the learning trial (T1). Vardenafil (1 mg/kg in $1 \mathrm{ml} / \mathrm{kg}$ ) was given orally (PO), 4 min after the T1. Dose, vehicle and administration time/route were determined based on earlier studies where these drugs showed clear memory-enhancing effects in the same ORT set-up (Rutten et al., 2006; Rutten et al., 2007).

\section{BEHAVIORAL TESTING}

We used the ORT as described previously (Prickaerts et al., 2002). Animals were tested in a circular arena (diameter $83 \mathrm{~cm}$ ) in which 2 objects were presented to them. A test session consisted of two 3 min trials, separated by a retention interval. In the learning trial (T1) 2 similar objects were presented to the animals and in the test trial (T2) these 2 objects were replaced by one identical (familiar) copy and a different (novel) object. We tested each different treatment on a variety of retention intervals, $1 \mathrm{~h}, 4 \mathrm{~h}, 8 \mathrm{~h}$, $10 \mathrm{~h}, 12 \mathrm{~h}$ and $24 \mathrm{~h}$. Four different sets of 3 identical objects were used, a set of glass $1 \mathrm{~L}$ bottles, a set of iron cubes, a set of grey cones made of polyvinyl chloride (PVC) 
and a set of bullet-shaped aluminum blocks. Animals were unable to move the objects (for more details about the objects and ORT procedure see Akkerman et al., 2012a).

Experimental conditions were semi-randomly assigned to experimental days which were separated by a wash-out period of at least a $24 \mathrm{~h}$ to prevent drug/dose interactions. 48 rats were divided into 2 cohorts of 24 animals. Within the cohorts a maximum of 3 different conditions could be tested on each experimental day, because 8 animals are required per condition in order to rule out side- and object biases (for details see Akkerman et al., 2012a). The first cohort of 24 animals performed each retention interval without receiving any treatment (untreated condition) and was subsequently tested in combination with vardenafil treatment (vardenafil condition). The second cohort performed each retention interval with vehicle treatment (vehicle condition) and also with rolipram treatment (rolipram condition). All treatment conditions are schematically presented in Table 1. As vehicle treated animals were part of the rolipram cohort, vehicle $(1 \mathrm{ml} / \mathrm{kg})$ was administered $\mathbb{P}$, $3 \mathrm{~h}$ after T1. Hence, no vehicle injection was given to these animals in the $1 \mathrm{~h}$ retention interval and the treatment was in fact the same as that of the $1 \mathrm{~h}$ untreated condition in the vardenafil cohort. However, this retention interval was designated as the $1 \mathrm{~h}$ vehicle condition to include it in the overall analysis. Of note, the untreated and vehicle treated animals had similar memory performance on every retention interval (see results section).

Table 1 Experimental condition

\begin{tabular}{lllllllll}
\hline Animals & Cohort & Treatments & \multicolumn{7}{l}{ ORT retention intervals } \\
\hline \multirow{4}{*}{48} & \multirow{2}{*}{$1(\mathrm{n}=24)$} & untreated & $1 \mathrm{~h}$ & $4 \mathrm{~h}$ & $8 \mathrm{~h}$ & $10 \mathrm{~h}$ & $12 \mathrm{~h}$ & $24 \mathrm{~h}$ \\
& & vardenafil & $1 \mathrm{~h}$ & $4 \mathrm{~h}$ & $8 \mathrm{~h}$ & $10 \mathrm{~h}$ & $12 \mathrm{~h}$ & $24 \mathrm{~h}$ \\
& \multirow{2}{*}{$2(\mathrm{n}=24)$} & vehicle & $1 \mathrm{~h} *$ & $4 \mathrm{~h}$ & $8 \mathrm{~h}$ & $10 \mathrm{~h}$ & $12 \mathrm{~h}$ & $24 \mathrm{~h}$ \\
& & rolipram & $\mathrm{x}$ & $4 \mathrm{~h}$ & $8 \mathrm{~h}$ & $10 \mathrm{~h}$ & $12 \mathrm{~h}$ & $24 \mathrm{~h}$ \\
\hline
\end{tabular}

This table gives an overview of the experimental conditions. All animals were fully habituated to the ORT procedures before the start of the experiment. 48 animals were divided over 2 cohorts. Each cohort received 2 different treatments which were tested in 6 separate retention intervals. In the untreated condition rats did not receive any injections. Vardenafil was administrated PO, 4 min after T1. Rolipram was administered IP $3 \mathrm{~h}$ after T1, therefore no $1 \mathrm{~h}$ interval was tested with rolipram. Vardenafil and rolipram were dissolved in the same vehicle. For the vehicle condition IP injections were given $3 \mathrm{~h}$ after T1.

* Therefore, no vehicle was administered in the $1 \mathrm{~h}$ retention interval. 


\section{STATISTICAL ANALYSIS}

The times rats spent exploring each object was measured. Time spent exploring the sample/familiar and the novel objects will be represented by ' $a$ ' and 'b', respectively. The following variables were calculated; $e 1=a_{1}+a_{2}$, e2 $=a_{3}+b$, and the relative discrimination index, $d 2=\left(b-a_{3}\right) / e 2$.

The effects of vehicle treatment were compared to the untreated condition using a repeated measures ANOVA (Interval(6) x Treatment(2)). Subsequently, the effects of vardenafil and rolipram treatments were analyzed using an Interval(6) $x$ Treatment(4) ANOVA. Differences between treatment conditions on $e 1, e 2$ and $d 2$ measures were analyzed for every separate retention interval using one-way ANOVA. LSD $t$-tests $(p<$ 0.05 ) were used to compare conditions per time point. Furthermore, one-sample $t$ statistics were performed on the $d 2$ measure to assess whether there was a difference from zero, which indicates recollection of the sample object (for more details see Akkerman, Prickaerts, Steinbusch, \& Blokland, 2012b).

\section{RESULTS}

\section{TIME DEPENDENT FORGETTING}

When comparing vehicle and untreated test conditions, no significant interaction effects were found on the exploration and discrimination measures $\left(F^{\prime} s(5,275)<1.16\right.$, $\left.p^{\prime} s>0.33\right)$. There was a significant main effect of interval on e1 $(F(5,275)=3.72, p<$ 0.003). Post-hoc analysis of the intervals showed that e1 was increased at the $12 \mathrm{~h}$ interval compared to all other intervals. There was also a main effect of treatment on e1 $(F(1,275)=109.20, p<0.001)$ and e2 $(F(1,275)=101.50, p<0.001)$. Post-hoc comparison of the treatments revealed that both e1 and e2 of the vehicle treated animals were significantly higher compared to untreated animals. This appears to be a general difference between the two cohorts.

Regarding the $d 2$ measure, which is depicted in Figure 1 , a significant main effect was found for Interval $(F(5,275)=16.21, p<0.001)$ but not for Treatment $(F(1,275)=$ 
0.013, n.s.), which shows that vehicle administration had no effect on memory performance. Post-hoc analysis showed that the $d 2$ measure of both treatment conditions significantly decreased from the $1 \mathrm{~h}$ to the $8 \mathrm{~h}$ interval and remained at the same level after longer intervals. The $d 2$ of untreated animals (data not shown) as well as vehicle treated animals was significantly higher than zero after the $1 \mathrm{~h}$ and $4 \mathrm{~h}$ retention interval ( $t$ 's $>4.26, p^{\prime} s<0.002$ ). When the retention delay was $8 \mathrm{~h}$ or longer the $d 2$ index was equal to zero, i.e. chance level, in both experimental conditions, indicating no recognition of the familiar object in T2. Taken together these findings indicate that natural memory extinguished between $4-8 \mathrm{~h}$ and vehicle treatment had no effect on forgetting.

\section{TIME DEPENDENT EFFECTS OF PDE-IS ON MEMORY}

Main effects of Interval $(F(5,526)=5.56, p<0.001)$ and Treatment $(F(3,526)=60.57$, $p<0.001)$ were found for e1. An interaction effect between Interval and Treatment was present for e2 $(F(14,526)=2.60 p=0.001)$. One way ANOVA's on each separate interval showed no differences in e1 and e2 between the untreated and vardenafil treatment condition or between the vehicle and rolipram treated condition (data not shown). This again reflects the general difference in exploration levels between both cohorts. The average exploration difference between both cohorts over all intervals was: $10.66 \mathrm{~s}$ in T1 and $10.50 \mathrm{~s}$ in T2. Despite this difference both cohorts showed abundant exploration for reliable object discrimination, i.e. on average e2 was higher than $19 \mathrm{~s}$ in any of the tested conditions.

One sample $t$-tests revealed that the $d 2$ (Figure 1) of all treatment conditions were significantly higher than zero on the 2 shortest intervals $\left(1 \mathrm{~h}\right.$ and $4 \mathrm{~h} ; \mathrm{t}^{\prime} \mathrm{s}>3.50, p^{\prime} \mathrm{s}<$ $0.002)$, indicating that all animals remembered the objects over these intervals. On the $8 \mathrm{~h}$ interval none of the treatment groups had a $d 2$ higher than zero $\left(t^{\prime} s<0.47\right.$, n.s.) which means that none of the groups showed object memory $8 \mathrm{~h}$ after learning. On the $10 \mathrm{~h}, 12 \mathrm{~h}$ and $24 \mathrm{~h}$ retention intervals the $d 2$ of untreated and vehicle treated animals remained equal to zero ( $t$ 's $<1.18$, n.s.). Interestingly, $d 2$ values in the vardenafil and rolipram treated animals returned above chance level again from the $10 \mathrm{~h}$ interval onwards ( $t$ 's $\left.>3.57, p^{\prime} s<0.002\right)$, demonstrating that object memory has 
reappeared $10 \mathrm{~h}$ after learning in the PDE-I treated animals. The mean $\mathrm{d} 2$ is graphically presented in Figure 1.

The Interval $x$ Treatment ANOVA showed that interaction was present on the $d 2$ measure $(F(14,526)=3.05, p<0.001)$, which is graphically presented in Figure 1 . Separate one way ANOVA's, performed on each retention interval, showed no differences between any of the 4 treatment conditions on the 3 shortest intervals ( $1 \mathrm{~h}$, $4 \mathrm{~h}$ and $8 \mathrm{~h} ; \mathrm{F}^{\prime} \mathrm{s}<0.53, \mathrm{n} . \mathrm{s}$.). On the other hand, significant differences were present at intervals of $10 \mathrm{~h}$ or longer ( $F^{\prime} s>2.70, p^{\prime} s<0.05$ ). Post-hoc comparisons showed that the $d 2$ values of both the vardenafil and rolipram treatment conditions were higher than the vehicle treated condition at all of these test intervals. Compared to the untreated condition the $d 2$ of both PDE-I treated conditions was also increased at the $12 \mathrm{~h}$ and $24 \mathrm{~h}$ intervals, but not the $10 \mathrm{~h}$ interval. No differences were found between vardenafil and rolipram treated animals or between untreated and vehicle treated animals, on any of the intervals. The $d 2$ indices of the different treatment conditions are graphically presented in Figure 1.

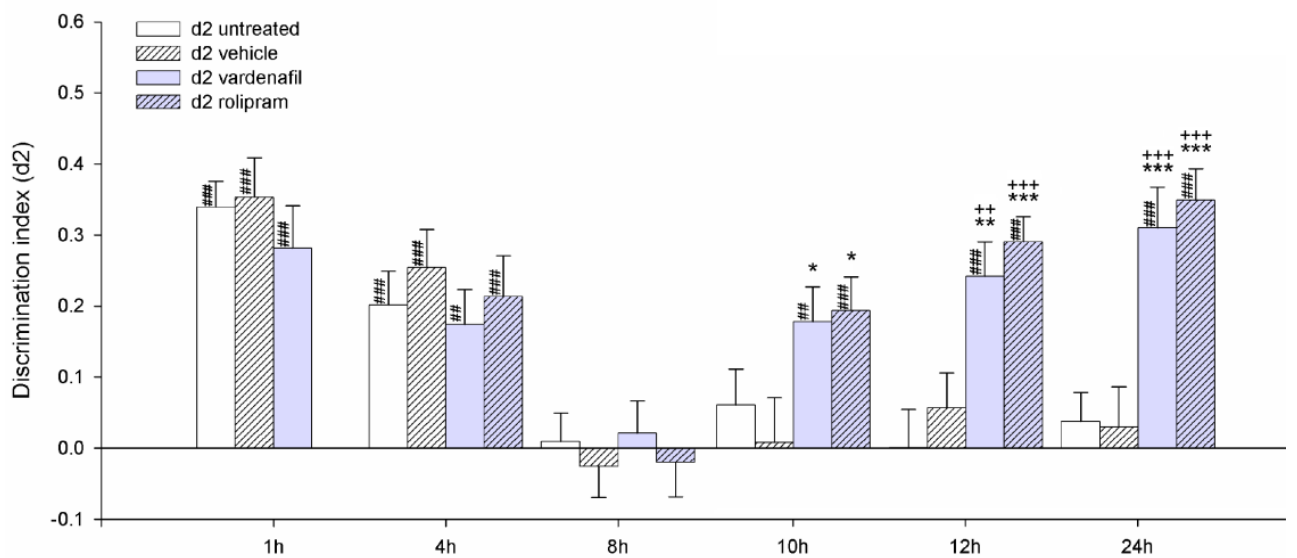

Figure 1 The effects of vardenafil and rolipram treatment on object discrimination

Discrimination performance $(\mathrm{d} 2)$ of untreated animals, and animals treated with vehicle, vardenafil (1 $\mathrm{mg} / \mathrm{kg})$ or rolipram $(0.03 \mathrm{mg} / \mathrm{kg})$ on different retention intervals in the ORT. Differences from zero are indicated with hashes (one-sample t-tests: \#\# $p<0.01$; \#\#\# $p<0.001$ ). Treatments were compared within each ORT retention interval. Asterisks indicate a difference from vehicle treated animals (LSD tests: * $p<$ $0.05 ; * * p<0.01 ; * * * p<0.001$ ), plus signs indicate a difference from untreated animals (LSD tests: $++p$ $<0.01$; +++ $p<0.001$ ). No significant differences were found between the vardenafil and rolipram conditions or between the untreated and vehicle conditions, on any of the retention intervals. 


\section{DISCUSSION}

Our study shows that solitary housed male Wistar rats are able to discriminate between a novel and an old object up to $4 \mathrm{~h}$ after learning but were unable to do so after an interval of $8 \mathrm{~h}$ or longer. This is in line with previous findings applying the test protocol as described here (Akkerman et al., 2012a). We found no discrimination differences between untreated animals and animals treated with vehicle, which indicates that the vehicle did not affect normal forgetting. In line with previous studies it was shown that vardenafil and rolipram improve memory performance when the animals are tested after $24 \mathrm{~h}$ (Prickaerts et al., 2002; Rutten et al., 2006; Rutten et al., 2007; Reneerkens et al., 2012). However, when the PDE-I treated animals were tested after $8 \mathrm{~h}$ they did not discriminate between the objects, indicating that the animals did not appear to remember the familiar object at this retention interval. Only after 10 $h$ the memory enhancing effects of the drugs emerged and these effects were still present after $24 \mathrm{~h}$. Since PDE inhibitors improve consolidation processes, these findings suggest that it takes time before information is more stably stored in neural circuits and can be measured in behavior. To our knowledge, this is the first time that it has been shown that the cognition enhancing effects of a drug is dependent on the retention delay at which the animal is tested.

The delay in memory manifestation shows similarities with the so-called Kamin-effect (Kamin, 1957). In 1957, Leon Kamin discovered that the behavioral expression of long term memories in rats is not stable over time as expression was impaired at intermediate retention intervals of approximately $1 \mathrm{~h}$. This phenomenon is thought to be the result of a transient retention failure or to reflect the decay in the STM trace before the LTM trace is available (Klein \& Spear, 1970; Sutton \& Carew, 2002). The Kamin-effect has been found in particular in aversively reinforced learning paradigms like passive avoidance and fear conditioning (e.g. Kamin, 1957; Destrade \& Cardo, 1974; Jaffard, Destrade, Soumireu-Mourat, \& Cardo, 1974; Tang, McNally, \& Richardson, 2007). It has been found in rats, mice, octopus, aplysia, chicks and goldfish (Sutton, Masters, Bagnall, \& Carew, 2001), implying that it is a universal memory characteristic. To our knowledge, no Kamin-effect has yet been 
demonstrated in spontaneous object memory, i.e. independent of negative reinforcement. In the current study we observed a Kamin-like effect that was pharmacologically induced. There appears to be a 'gap' in long term object memory itself at about $8 \mathrm{~h}$ after learning and not, like the Kamin effect, at $1 \mathrm{~h}$ post-learning, in between STM and LTM. Even though the observed decay and restoration of memory in the present study shows similarities with the Kamin effect the underlying mechanisms of the time-dependent drug effects could be very different compared to the mechanisms underlying the Kamin effect.

The 'gap' in object discrimination performance was identical for both compounds, with object discrimination reappearing after a $10 \mathrm{~h}$ interval in vardenafil and rolipram treated animals and lasting up to at least $24 \mathrm{~h}$ after learning. On basis of our previous studies we have suggested that CGMP is involved in early-phase consolidation and CAMP in late-phase consolidation as vardenafil is effective when administered shortly after acquisition, while rolipram is only effective when given $3 \mathrm{~h}$ after learning (Prickaerts et al., 2002; Rutten et al., 2006; Rutten et al., 2007). To our knowledge there are currently no studies that have assessed the pharmacokinetics of vardenafil and rolipram in rats using a similar administration route as our current study. However, based on previous studies it can be estimated that the half-lives of vardenafil (PO) and rolipram (IP) in rats are approximately 30 and $15 \mathrm{~min}$, respectively (Krause \& Kuhne, 1988; Walker et al. 1999). Given these very short half-lives, one can assume that CGMP and CAMP signaling was only enhanced for a short period after injection. Despite of the difference in timing, both treatments produced the same temporal (object) memory profile which might indicate that CGMP and CAMP are both part of the same signaling cascade underlying LTM formation. Apparently, the subsequent expression of genes and proteins and coherent network changes needed for long-term storage of information requires at least $10 \mathrm{~h}$, in our ORT paradigm, to become fully effective. It appears that two distinct types of memory are at play. A robust LTM trace that requires time to develop and a transient STM/intermediate memory (IM) trace that declines in 1 - 8 h (Kamin, 1957; Sutton \& Carew, 2002; Rudy \& Wright-Hardesty, 2005; Tang et al., 2007). 
In the current study, we found a substantial difference in exploration between the untreated/vardenafil cohort and the vehicle/rolipram cohort. However, no differences were found between the conditions within each cohort and the exploration difference between the cohorts was almost identical in e1 and e2. Therefore, the exploration differences are very likely due to a cohort effect and not the treatments. Despite these exploration differences, it was still possible to compare between treatment conditions of both cohorts in an unbiased manner because the $d 2$ measure corrects for differences in exploration. Moreover, it has been shown that there is no correlation between exploration levels in T1 or T2 with the $d 2$ measure (Akkerman et al., 2012b). Thus, longer exploration of objects does not lead to a better memory performance in T2.

Several stress-related explanations have also been postulated to explain the Kamineffect, including incubation of fear, corticosteroids affecting memory processes and a failure to retrieve the memory trace (Klein \& Spear, 1970). Although it is well known that memory processes can be strongly affected by corticosteroids (Roozendaal, Hahn, Nathan, de Quervain, \& McGaugh, 2004; Roozendaal, Castello, Vedana, Barsegyan, \& McGaugh, 2008), our finding of a time-dependent drug effect in the ORT makes stress or fear are unlikely factors to explain the time-dependent memory 'gap' (Klein \& Spear, 1970). The ORT is considered to be a low stress-task because it uses innate tendency of the animal to investigate novelty instead of negative reinforcement (Ennaceur \& Delacour, 1988).

Another explanation is offered by the complementary learning systems (CLS) framework. According to this theory the brain requires two differently specialized learning systems. On the one hand there is the hippocampal system responsible for sparse, rapid learning of episodic memories and on the other hand an overlapping neocortical memory system for gradually integrating information across episodes to extract latent semantic structure (McClelland, McNaughton, \& O'Reilly, 1995; O'Reilly, Bhattacharyya, Howard, \& Ketz, 2011). This CLS framework can be extrapolated to the ORT in which the hippocampus is considered as the site where object information from the perirhinal cortex is integrated with contextual information from the 
parahippocampal (or in rats, postrhinal) cortex, thus underlying the formation of episodic memory which is eventually stored in the neocortex (e.g. Winters \& Bussey, 2005; Eichenbaum, Yonelinas, \& Ranganath, 2007). Interestingly, both PDE4 and PDE5 are also found in the hippocampus and neocortex (Lakics, Karran, \& Boess, 2010).

It has been found that spontaneous sharp wave/ripple (SWR) events appear in the hippocampal and neocortical EEG during passive wakefulness and slow wave sleep (SWS) (Kudrimoti, Barnes, \& McNaughton, 1999; Sutherland \& McNaughton, 2000). These activation patterns appear to represent the reactivation/consolidation of past events and of episodic memories into more stable neocortical representations, i.e. LTM. Thus, the transition from transient memory to a more stable neocortical memory may be substantiated during reactivation (Rasch \& Born, 2007). Rats are nocturnal animals and sleep during daytime, for this reason our animals were kept under an inversed day/night cycle. Lights were automatically switched off from 6 AM until 7 PM and the animals were tested during this $13 \mathrm{~h}$ dark period. Most SWR events are likely to have occurred during SWS in the subsequent $11 \mathrm{~h}$ lights-on period. On the other hand, it is also plausible that SWR events occurred during the dark period as the animals frequently displayed passive/immobile periods during their 'active' time of the day. Since many different retention intervals were tested in the current study, it may seem plausible that sleep could have played a cofounding role in the current study. However, all retention intervals from $1 \mathrm{~h}-12 \mathrm{~h}$ were tested within the $13 \mathrm{~h}$ dark period of the day. Only in between the $12 \mathrm{~h}$ and $24 \mathrm{~h}$ interval was an 'inactive', i.e. lights-on, period of $11 \mathrm{~h}$. Our results show that the memory enhancing effect of the PDE-I treatments already becomes apparent during the active/dark period, $10 \mathrm{~h}$ after learning. Hence, it appears that the observed memory enhancement is independent of long undisturbed periods of sleep and that the passive/immobile periods during the dark phase provided ample opportunity for putative, PDE-I stimulated memory reactivation processes to form long-term neocortical memory representations.

In summary, we found that the positive effect of both PDE inhibitors on object recognition memory is time-dependent. It is possible that two distinct memory 
representations are involved. One rapid transient form that degrades within $8 \mathrm{~h}$ after learning and a stable form integrating episodic information into the existing semantic structure, which gradually builds at a neurobiological level and becomes manifest only after $10 \mathrm{~h}$ in this particular ORT protocol. Both CGMP and CAMP appear to be sequentially involved in the development of the latter memory form. Therefore, the underlying neurobiological mechanism might be linked to consolidation/reactivation processes of transient episodic events into more stable memory representations. A major implication of the present findings is that possible positive effects of drugs on long-term memory might be overlooked due to the time-dependency of their effects, i.e. subjects are tested too soon after acute treatment to measure a full effect on LTM. It might therefore be recommendable to always test LTM after intervals of at least 12$24 \mathrm{~h}$. 


\section{REFERENCES}

Abel, T., \& Lattal, K.M. (2001). Molecular mechanisms of memory acquisition, consolidation and retrieval. Current Opinion in Neurobiology, 11(2), 180-187.

Akkerman, S., Blokland, A., Reneerkens, O., van Goethem, N.P., Bollen, E., Gijselaers, H.J.M. (2012). Object recognition testing: Methodological considerations on exploration and discrimination measures. Behav Brain Res, 232(2), 335-347.

Akkerman, S., Prickaerts, J., Steinbusch, H.W.M., \& Blokland, A. (2012). Object recognition testing: Statistical considerations. Behav Brain Res, 232(2), 317322.

Bernabeu, R., Schroder, N., Quevedo, J., Cammarota, M., Izquierdo, I., \& Medina, J.H. (1997). Further evidence for the involvement of a hippocampal cGMP/cGMPdependent protein kinase cascade in memory consolidation. Neuroreport, 8(9-10), 2221-2224.

Bruno, O., Fedele, E., Prickaerts, J., Parker, L.A., Canepa, E., Brullo, C., Cavallero, A., Gardella, E., Balbi, A., \& Domenicotti, C. (2011). GEBR-7b, a novel PDE4D selective inhibitor that improves memory in rodents at non-emetic doses. $\mathrm{Br} J$ Pharmacol, 164(8): 2054-2063.

Destrade, C., \& Cardo, B. (1974). Effects of post-trial hippocampal stimulation on time-dependent improvement of performance in mice. Brain Research, 78(3), 447-454.

Devan, B.D., Sierra-Mercado, D., Jr., Jimenez, M., Bowker, J.L., Duffy, K.B., Spangler, E.L., \& Ingram, D.K. (2004). Phosphodiesterase inhibition by sildenafil citrate attenuates the learning impairment induced by blockade of cholinergic muscarinic receptors in rats. Pharmacol Biochem Behav, 79(4): 691-699.

Eichenbaum, H., Yonelinas, A.P., \& Ranganath, C. (2007). The medial temporal lobe and recognition memory. Annu Rev Neurosci, 30, 123-152.

Ennaceur, A. (2010). One-trial object recognition in rats and mice: Methodological and theoretical issues. Behavioural Brain Research, 215(2): 244-254.

Ennaceur, A., \& Delacour, J. (1988). A new one-trial test for neurobiological studies of memory in rats. 1: Behavioral data. Behav Brain Res, 31(1), 47-59.

Jaffard, R., Destrade, C., Soumireu-Mourat, B., \& Cardo, B. (1974). Time-dependent improvement of performance on appetitive tasks in mice. Behavioral Biology, 11(1), 89-100.

Kamin, L.J. (1957). The retention of an incompletely learned avoidance response. Journal of Comparative and Physiological Psychology, 50, 457-460.

Klein, S.B., \& Spear, N.E. (1970). Forgetting by the rat after intermediate intervals ("Kamin effect") as retrieval failure. Journal of Comparative and Physiological Psychology, 71(1), 165-170. 
Krause, W., \& Kuhne, G. (1988). Pharmacokinetics of rolipram in the rhesus and cynomolgus monkeys, the rat and the rabbit. Studies on species differences. Xenobiotica, 18, 561-571.

Kudrimoti, H.S., Barnes, C.A., \& McNaughton, B.L. (1999). Reactivation of hippocampal cell assemblies: effects of behavioral state, experience, and EEG dynamics. J Neurosci, 19(10), 4090-4101.

Lakics, V., Karran, E.H., \& Boess, F.G. (2010). Quantitative comparison of phosphodiesterase mRNA distribution in human brain and peripheral tissues. Neuropharmacology, 59(6), 367-374.

Levallet, G., Hotte, M., Boulouard, M., \& Dauphin, F. (2009). Increased particulate phosphodiesterase 4 in the prefrontal cortex supports 5-HT4 receptorinduced improvement of object recognition memory in the rat. Psychopharmacology (Berl), 202(1-3): 125-139.

McClelland, J.L., McNaughton, B.L., \& O'Reilly, R.C. (1995). Why there are complementary learning systems in the hippocampus and neocortex: insights from the successes and failures of connectionist models of learning and memory. Psychol Rev, 102(3), 419-457.

O'Reilly, R.C., Bhattacharyya, R., Howard, M.D., \& Ketz, N. (2011). Complementary Learning Systems. Cogn Sci.

Prickaerts, J., Sik, A., van der Staay, F.J., de Vente, J., \& Blokland, A. (2005). Dissociable effects of acetylcholinesterase inhibitors and phosphodiesterase type 5 inhibitors on object recognition memory: acquisition versus consolidation. Psychopharmacology (Berl), 177(4), 381-390.

Prickaerts, J., van Staveren, W.C.G., Sik, A., Markerink-van Ittersum, M., Niewöhner, U., van der Staay, F.J. (2002). Effects of two selective phosphodiesterase type 5 inhibitors, sildenafil and vardenafil, on object recognition memory and hippocampal cyclic GMP levels in the rat. Neuroscience, 113(2), 351-361.

Rasch, B.r., \& Born, J. (2007). Maintaining memories by reactivation. Current Opinion in Neurobiology, 17(6), 698-703.

Reneerkens, O.A., Rutten, K., Akkerman, S., Blokland, A., Shaffer, C.L., Menniti, F.S. (2012). Phosphodiesterase type 5 (PDE5) inhibition improves object recognition memory: indications for central and peripheral mechanisms. Neurobiol Learn Mem, 97(4), 370-379.

Roozendaal, B., Castello, N.A., Vedana, G., Barsegyan, A., \& McGaugh, J.L. (2008). Noradrenergic activation of the basolateral amygdala modulates consolidation of object recognition memory. Neurobiol Learn Mem, 90(3), 576-579.

Roozendaal, B., Hahn, E.L., Nathan, S.V., de Quervain, D.J., \& McGaugh, J.L. (2004). Glucocorticoid effects on memory retrieval require concurrent noradrenergic activity in the hippocampus and basolateral amygdala. J Neurosci, 24(37), 8161-8169. 
Rudy, J.W., \& Wright-Hardesty, K. (2005). The temporal dynamics of retention of a context memory: Something is missing. Learning \& Memory, 12(2), 172-177.

Rutten, K., Prickaerts, J., \& Blokland, A. (2006). Rolipram reverses scopolamineinduced and time-dependent memory deficits in object recognition by different mechanisms of action. Neurobiol Learn Mem, 85(2), 132-138.

Rutten, K., Prickaerts, J., Hendrix, M., van der Staay, F.J., Sik, A., \& Blokland, A. (2007). Time-dependent involvement of CAMP and CGMP in consolidation of object memory: studies using selective phosphodiesterase type 2, 4 and 5 inhibitors. Eur J Pharmacol, 558(1-3), 107-112.

Sutherland, G.R., \& McNaughton, B. (2000). Memory trace reactivation in hippocampal and neocortical neuronal ensembles. Current Opinion in Neurobiology, 10(2), 180-186.

Sutton, M.A., \& Carew, T.J. (2002). Behavioral, Cellular, and Molecular Analysis of Memory in Aplysia I: Intermediate-Term Memory. Integrative and Comparative Biology, 42(4), 725-735.

Sutton, M.A., Masters, S.E., Bagnall, M.W., \& Carew, T.J. (2001). Molecular Mechanisms Underlying a Unique Intermediate Phase of Memory in Aplysia. Neuron, 31(1), 143-154.

Tang, H.H.Y., McNally, G.P., \& Richardson, R. (2007). The Effects of FG7142 on Two Types of Forgetting in 18-Day-Old Rats. Behavioral Neuroscience, 121(6), 1421-1425.

Walker, D.K., Ackland, M.J., James, G.C., Muirhead, G.J., Rance, D.J., Wastall, P., Wright, P.A. (1999). Pharmacokinetics and metabolism of sildenafil in mouse, rat, rabbit, dog and man. Xenobiotica, 29, 297-310.

Winters, B.D., \& Bussey, T.J. (2005). Transient Inactivation of Perirhinal Cortex Disrupts Encoding, Retrieval, and Consolidation of Object Recognition Memory. The Journal of Neuroscience, 25(1), 52-61. 



\section{Chapter 7}




\section{PDE5 INHIBITION IMPROVES OBJECT}

MEMORY IN STANDARD HOUSED RATS BUT NOT IN RATS HOUSED IN AN ENRICHED ENVIRONMENT: IMPLICATIONS FOR MEMORY MODELS?

S. Akkerman, J. Prickaerts, A.K. Bruder, K.H.M. Wolfs, J. De Vry, T. Vanmierlo and A. Blokland 


\section{ABSTRACT}

Drug effects are usually evaluated in animals housed under maximally standardized conditions. However, it is assumed that an enriched environment (EE) more closely resembles human conditions as compared to maximally standardized laboratory conditions. In the present study, we examined the acute cognition enhancing effects of vardenafil, a PDE5 inhibitor, which stimulates protein kinase G/CREB signaling in cells, in three different groups of male Wistar rats tested in an object recognition task (ORT). Rats were either housed solitarily (SOL) or socially (SOC) under standard conditions, or socially in an EE. Although EE animals remembered object information longer in the vehicle condition, vardenafil only improved object memory in SOL and SOC animals. While EE animals had a heavier dorsal hippocampus, we found no differences between experimental groups in total cell numbers in the dentate gyrus, CA2-3 or CA1. Neither were there any differences in markers for pre- and postsynaptic density. No changes in PDE5 mRNA- and protein expression levels were observed. Basal pCREB levels were increased in EE rats only, whereas $\beta$-catenin was not affected, suggesting specific activation of the MAP kinase signaling pathway, and not the AKT pathway. A possible explanation for the inefficacy of vardenafil could be that CREB signaling is already optimally stimulated in the hippocampus of EE rats. Since previous data has shown that acute PDE5 inhibition does not improve memory performance in humans, the use of EE animals could be considered as a more valid model for testing cognition enhancing drugs. 


\section{INTRODUCTION}

Many pharmacological animal studies have shown the beneficial effects that drugs can have on memory performance. However, the effects of these drugs are generally only modest, or even absent, when tested in humans. Many factors could explain this possible discrepancy in the effectiveness of drugs on memory performance (Sarter, 2006). It could be argued that the test paradigms used in animal research have poor translational value. Additionally, laboratory rodents may not be suitable for testing cognition enhancing drugs because the impoverished environment in which the animals are raised does not enable the kind of natural brain development seen in humans. Testing of drugs that improve brain function should ideally be done in subjects that have a 'normal' brain development (Gurwitz, 2001; Lynch \& Gall, 2013; van Praag, Barlow, \& Gage, 2001; Vedovelli, et al., 2011). One way to achieve this is by testing drugs in animals raised in an enriched environment (EE), which has been shown to markedly reduce abnormal repetitive behaviors without affecting the precision or reproducibility of results (Wolfer, et al., 2004).

From the early studies of Rosenzweig, it is known that environmental enrichment in rats has clear effects on brain development. The 'EE' brain has is heavier and has more neuronal connections compared to brains of standard housed animals (Rosenzweig \& Bennett, 1972). Related to this, several morphological changes, associated with memory performance, have been observed after EE. For example, EE has been shown to; increase neurogenesis in the dendate gyrus (Bruel-Jungerman, Laroche, \& Rampon, 2005; Kempermann, Kuhn, \& Gage, 1997), increase neuronal cell volume, dedritic length/branching/spine density in the CA1 pyramidal neurons (Faherty, Kerley, \& Smeyne, 2003; Moser, Trommald, \& Andersen, 1994) and increases in pre- and postsynaptic proteins in the forebrain, hippocampus, thalamus and hypothalamus (Nithianantharajah, Levis, \& Murphy, 2004). Not surprisingly, it is well documented that enriched animals generally outperform animals that are raised under 'standard' housing conditions on a wide variety of cognitive behavioral tests (Diniz, et al., 2010; Galani, et al., 2007; Harati, et al., 2013; Harati, et al., 2011; Hebb, 1947; Simpson \& Kelly, 2011). EE has been shown to affect multiple neurotransmitter systems, 
including the dopaminergic (Bowling, Rowlett, \& Bardo, 1993), serotonergic (Hellemans, Nobrega, \& Olmstead, 2005), glutamatergic (Andin, Hallbeck, Mohammed, \& Marcusson, 2007), and the cholinergic system (Thouvarecq, Caston, \& Protais, 2007), which are heavily involved in learning and memory processes (Myhrer, 2003). It seems plausible to assume that drugs targeting these neurotransmitters, or their downstream targets, may have differential effects in animals that are living in different housing conditions. This assumption has been confirmed by studies showing that drugs targeting the dopaminergic (Del Arco, et al., 2007; Zeeb, Wong, \& Winstanley, 2013), serotonergic (Yildirim, Erol, \& Ulupinar, 2012), glutamatergic (Arndt, Arnold, \& Cain, 2014) systems, indeed, have differential effects in animals raised in an EE compared to standard housed animals. Although several studies have investigated $\mathrm{EE}$-drug interactions in various neurodegenerative and psychiatric animal models (Laviola, Hannan, Macri, Solinas, \& Jaber, 2008), the effects of EE on cognition enhancing drugs have not yet been thoroughly investigated.

In the present study, we examined the efficacy of vardenafil in EE rats and rats kept either solitary (SOL) or socially (SOC) in a standard environment. First, we used the object recognition task (ORT) to investigate the effects of the 3 different housing conditions on memory performance (c.f., Simpson \& Kelly, 2012). Subsequently, we tested the acute effect of different doses of the cognition enhancer vardenafil, on retention intervals of $24 \mathrm{~h}$ and $48 \mathrm{~h}$. Based on previous studies, we hypothesized that EE would enhance ORT performance (Bechara \& Kelly, 2013; Bruel-Jungerman, et al., 2005; Escorihuela, et al., 1995; Kazlauckas, et al., 2011; Leger, et al., 2012; Vedovelli, et al., 2011) and alter the efficacy of vardenafil treatment. After the behavioral testing, brain plasticity was assessed with morphological and neurochemical signaling markers. 


\section{METHODS}

\section{ANIMALS}

This study was approved by the local animal experimental committee of Maastricht University, the Dier Experimenten Commissie (DEC) Maastricht. Fifty-eight male Wistar rats were obtained from Harlan (Horst, The Netherlands) just after weaning (four weeks of age). Eighteen were housed in an enriched environment ( $E E, n=9 /$ cage), 18 were socially housed (SOC) ( $n=3 /$ cage) in standard polycarbonate cages (Tecniplast, type 2154F, dimensions $48 \times 26.5 \times 21 \mathrm{~cm}$, floor area $940 \mathrm{~cm} 2$ ), and 22 were individually housed in standard cages (SOL). The 3 different housing conditions are presented in Figure 1.

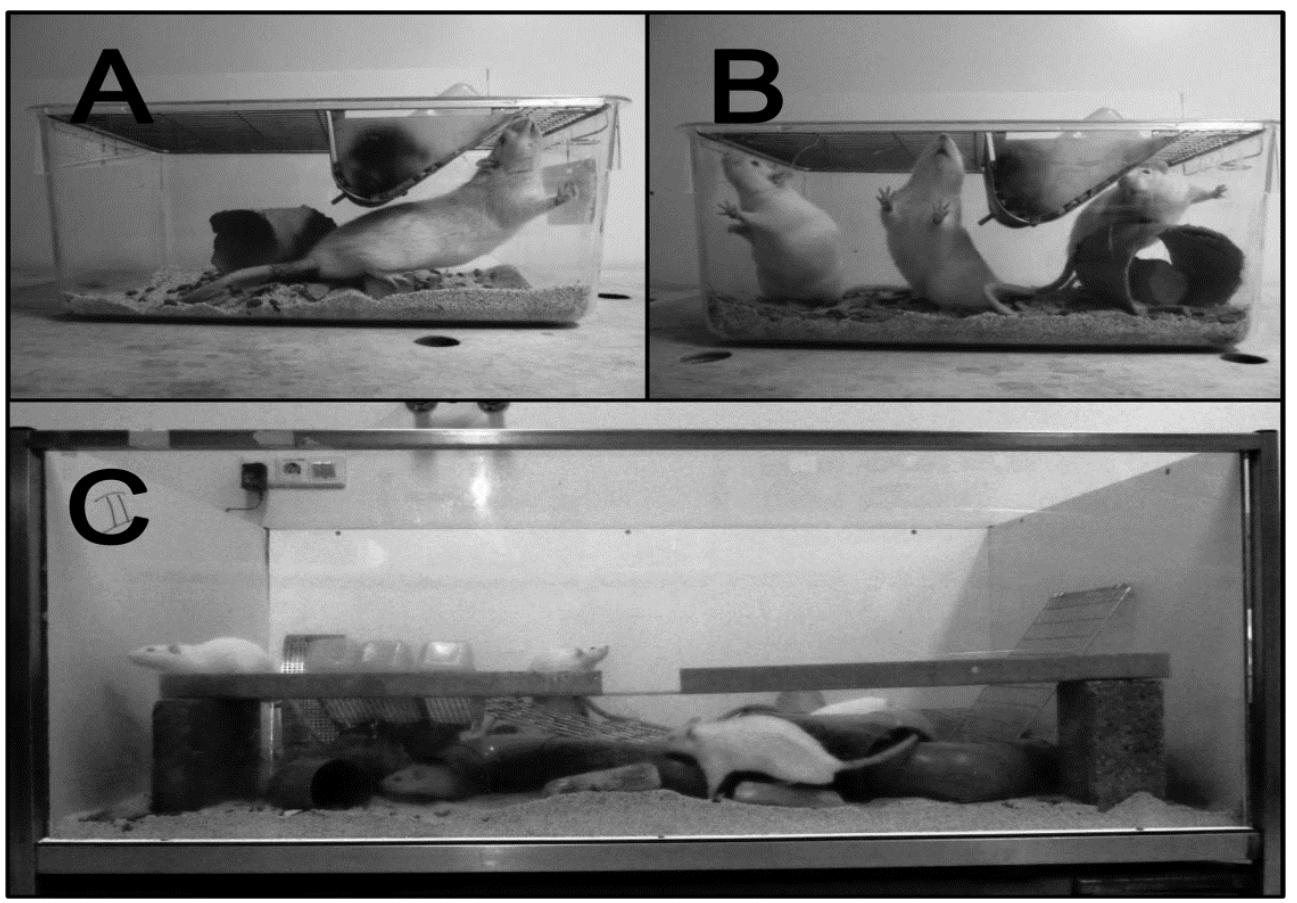

\section{Figure 1 Housing conditions}

Pictures of the 3 different housing conditions; (A) solitary housed animals (SOL); (B) socially housed animals (SOC); and (C) animals housed in an enriched environment (EE). Of note, the enriched environment picture only represents the object arrangement during one particular week, the constellation and number of items was changed weekly. 
The standard cages (Tecniplast, series -114) and contained a cardboard tube and a wooden block and had a stainless steel wire lid on top. The special EE cages measured $150 \times 90 \times 80 \mathrm{~cm}$ and contained a wide array of objects which were rearranged weekly. All cages contained sawdust bedding and animals had free access to food and water. Animals were housed and tested in the same room, under a reversed light/dark cycle (lights on from 19:00 to 7:00). A radio always played softly in the background, room temperature was $21^{\circ} \mathrm{C}$ and the average humidity was kept between 45-55\%. Testing was done during the dark phase, between 8:30 and 16.30. Of note, during testing EE animals were briefly (for about $1 \mathrm{~h}$ ) moved into a standard holding cage ( $n=3 /$ cage).

\section{Object Recognition TASK}

Behavioral testing started when the animals were 3 months old. An ORT session consisted of a learning- (T1) and a test-trial (T2), separated by a retention interval. In $\mathrm{T} 1$, two identical objects were placed in the arena and in $\mathrm{T} 2$ the arena contained a novel (different) object and a copy from T1. In both trials, the animals were allowed to freely explore the objects and the arena for 3 min during which the exploration of each object was scored manually on a personal computer. The relative discrimination index ( $d 2$ ) was used to assess memory performance. The $d 2$ measure is calculated from the exploration of the objects in the second trial ((time novel object - time familiar object) / (time novel object + time familiar object)). For an elaborate description of the ORT setup, objects and procedures, see Akkerman et al. ( 2012).

Before drug testing started, we examined the performance of the different groups at a $1 \mathrm{~h}$ and a $24 \mathrm{~h}$ retention interval, which typically relates to a good and poor performance, respectively (Akkerman, Prickaerts, Steinbusch, \& Blokland, 2012). During the experiment, a decision was made to additionally test the groups at a $48 \mathrm{~h}$ retention interval, as vehicle treated EE rats still showed object memory at the $24 \mathrm{~h}$ retention interval. 


\section{DRUg TREATMENT}

Vardenafil (kindly donated by Bayer AG, Wuppertal, Germany) was freshly suspended in $1 \%$ tylose (methyl-cellulose) in milli-Q, on each experimental day. Vardenafil was tested at different doses: 0 (vehicle), $0.03,0.1,0.3$ and $1 \mathrm{mg} / \mathrm{kg}$. Administrations were always PO (injection volume $2 \mathrm{ml} / \mathrm{kg}$ ) immediately after the first trial.

\section{DORSAL HIPPOCAMPAL WEIGHT}

The animals were sacrificed 2 weeks after behavioral testing. Half of the animals of each housing group were killed by decapitation and the dorsal hippocampus was dissected (on ice), weighed, and immediately frozen in liquid nitrogen. Samples were then stored at $-80^{\circ} \mathrm{C}$ and later used for $\mathrm{QPCR}$ and Western Blotting. We focused on the dorsal hippocampus as it is preferentially involved in memory processes, in contrast to the ventral hippocampus which is preferentially involved in emotional processes (Bannerman, et al., 2004). Furthermore, there are strong indications for involvement of the dorsal hippocampus in our version of the ORT (Prickaerts, de Vente, Honig, Steinbusch, \& Blokland, 2002).

\section{PeRfusions}

The other half of the animals was killed by intracardial perfusion under lethal pentobarbital anesthesia (100 mg/kg, IP). Ice cooled tyrode solution was used during the first minute of perfusion, followed, for $10 \mathrm{~min}$, by a fixative containing $4 \%$ paraformaldehyde, 15\% picric acid, and $0.05 \%$ glutaraldehyde in phosphate buffer( $0.1 \mathrm{M}$ ) (Schmitz, et al., 2004). Brains were dissected and post-fixated for 18 hours in Somogyi's fixation solution without the glutaraldehyde. For cryoprotection, the brains were immersed in a $10 \%$ sucrose solution in $0.1 \mathrm{M}$ phoshate-buffered elix for $24 \mathrm{~h}$, followed by $24 \mathrm{~h}$ immersion in a $20 \%$ sucrose solution in $0.1 \mathrm{M}$ phoshate-buffer. Subsequently, the brains were frozen using $\mathrm{CO}_{2}$ gas and cut into coronal sections (10 series, thickness $30 \mu \mathrm{m}$ ) at $-25^{\circ} \mathrm{C}$ using a CM3050 cryostat (Leica, Wetzlar, Germany). Sections were stored at $-80{ }^{\circ} \mathrm{C}$ until further processing for stereological measurements. 


\section{Histology AND SteREOLOGY}

Nissl staining was performed on one series of the coronal sections. Sections were put in a 1x TBS solution and mounted on coated glass slides. After mounting, the glass slides were left to air-dry overnight at room temperature. Sections were immersed in a $1 \%$ acetic acid, $0.04 \mathrm{M}$ sodium acetate solution (solution $\mathrm{A}$ ) for $20 \mathrm{~min}$, followed by immersion in a $75 \%$ ethanol, $1.25 \%$ triton $x-100$ (Solution B) solution for $20 \mathrm{~min}$, after which the sections were again immersed in solution $A$ for 20 min. Subsequently, sections were stained by immersion into solution $A$, containing $0.1 \%$ cresyl violet, for $10 \mathrm{~min}$. After staining, sections were washed three times by immersion into solution $A$ for $1 \mathrm{~min}$ and dehydrated via immersion into 100\% ethanol for $1 \mathrm{~min}$, thrice. Next, the sections were immersed into isopropanol two times for $5 \mathrm{~min}$, followed by immersion in xylene, twice for 5 min. Finally, the sections were closed in and the cover glasses were fixed using Depex.

The sections were investigated using design-based stereology (Schmitz \& Hof, 2005), a well established method for achieving reliable and accurate estimates of the volume of brain regions and the amount of neurons within it. For volume determination and cell counts of the dorsal hippocampal subregions, the Nissl stained sections ranging from bregma -2.52 to $-4.68 \mathrm{~mm}$ (Paxinos \& Watson, 1998) were analyzed under an Olympus AX70 bright field microscope (analySIS; Imaging System, Münster, Germany). On average, six sections (at $300 \mu \mathrm{m}$ intervals) were analyzed per animal, depending on the individual rostrocaudal extension of the dorsal hippocampus. Several subregions within the dorsal hippocampus were assessed; the dentate gyrus (DG), cornu ammonis 1 (CA1) and cornu ammonis 2 and 3 (CA2-3). Stereo Investigator software (Version 8.26, MBF Bioscience, Williston, VT, USA) was used to measure the surface area, section thickness, and estimate the cell population of each sub-region. Delineations of each sub-region were made using a $4 x$ objective (numerical aperture $[N A]=0.16)$ and cell counts were performed using a 100x oil lens (NA $=1.35$, Olympus UPlanApo). 


\section{QUANTITATIVE PCR}

RNA in the dorsal hippocampus was isolated from half the animals of each housing group. Quantitative PCR ( $q P C R$ ) of Pde5 was performed to compare the levels of PDE5 mRNA between the different housing conditions. RNA isolation was performed by TRIzol reagent ${ }^{\circledR}$ mediated extraction, according to the manufacturer's instructions (Life Technologies, Bleiswijk, The Netherlands). $500 \mathrm{ng}$ of total RNA was converted into cDNA using iscript Advanced cDNA Synthesis Kit for RT-qPCR (Bio-Rad Laboratories, Veenendaal, The Netherlands). Quantitative PCR of Pde5 and the housekeeping gene Hprt were performed with the IQ SYBR Green Supermix Kit (BioRad Laboratories, Veenendaal, The Netherlands), $0.2 \mu \mathrm{l}$ primer $(10 \mu \mathrm{M})$ was added to $4.6 \mu \mathrm{H} 2 \mathrm{O}$ and $10 \mu \mathrm{l} \mathrm{SYBR}$ Green per $5 \mu \mathrm{l}$ cDNA sample (2 $\mathrm{ng} / \mu \mathrm{l})$. The following sequences (Sigma-Aldrich, St.Louis, MI) were used:

Hprt, 5'TTGCTGGTGAAAAGGACCTC3'

Hprt-1, 5' TCCACTTTCGCTGATGACAC 3'

Pde5, 5' TGGTGACGTTAGAGGTCCTG3'

Pde5, 5'CGCTGTTTCCAGATCAGACA3'

Analysis of the data was performed via the delta-delta CT method and expression of Pde5 was normalized against Hprt.

\section{WESTERN BLOTTING}

Western Blots were used to assess protein levels of BDNF, PDE5, pCREB, $\beta$-catenin, pGSK-3 $\beta$, synaptophysin, PSD95, and ERK2 in the dorsal hippocampus of half of the animals of each housing condition. GAPDH and $\beta$-actin were used for normalization.

One hundred to $130 \mathrm{mg}$ dorsal hippocampus tissue was homogenized using a MiniBead Beater three times for $30 \mathrm{~s}$ in $1 \mathrm{ml}$ ice-cold homogenizing buffer (two phosphatase inhibitor tablets/20 ml (Roche, \#04906845001, Almere, The Netherlands) and one protease inhibitor tablet/20 ml (Roche \#11836153001, Almere, The Netherlands) were added to the homogenizing buffer $(100 \mathrm{mM}$ Tris, $200 \mathrm{mM} \mathrm{NaCl}$, 
$1 \mathrm{mM}$ EDTA, $2 \mathrm{mM}$ DTT, 0.05\% Triton (vol/vol)). Samples were centrifuged for $20 \mathrm{~min}$ at $4^{\circ} \mathrm{C}, 16000 \mathrm{~g}$ and the supernatant was stored at $-80^{\circ} \mathrm{C}$. Protein concentrations were determined using Bio-Rad Lowry Protein Assay (Bio-Rad Laboratories Inc., Hercules, USA).

Brain homogenates in sample buffer were boiled for five minutes and then separated on a $10 \%$ or $14 \%$ (mBDNF and proBDNF) SDS-PAGE gel (30 $\mu \mathrm{g}$ per sample). Following electrophoresis, proteins were transferred to a nitrocellulose membrane (Bio-Rad Laboratories, Hercules, USA), which was subsequently blocked with blocking buffer (50\% Odyssey blocking buffer in PBS, Li-Cor, Lincoln, USA) for one hour at room temperature. Next, the membranes were incubated overnight at $4{ }^{\circ} \mathrm{C}$ with the primary antibodies in blocking buffer: 1:600 rabbit anti-BDNF (H-117; Santa Cruz Biotechnology \#SC-20981, Heidelberg, Germany); 1:500 rabbit anti-PDE5A1 (Fabgennix \#PC-Pde5A, Frisco, TX), 1:100 rabbit anti-pCREB (Cell Signaling Technology \#9198S, Beverly, MA), 1:2000 mouse anti- $\beta$-catenin (BD Transduction laboratories \#20079, Franklin Lakes, NJ), 1:1000 rabbit anti-pGSK-3 3 (Cell Signaling Technology \#9336S, Beverly, MA), 1:1000 mouse anti-synaptophysin (Millipore MSxsynaptophysin MS\#MAB5258, Billerica, MA), 1:2000 mouse anti-PSD95 (QED Bioscience inc \#56452, San Diego, CA), 1:1000 rabbit anti-ERK2 (Cell signaling technology \#9108, Beverly, MA). For normalization, 1:2.000.000 mouse anti-Gapdh (Fitzgerald Industries \#10R-G109A, Huissen, The Netherlands) or 1:1000 mouse anti$\beta$-actin (Santa Cruz Biotechnologies \#F0110; Santa Cruz, CA) was used. After washing with phosphate-buffered saline- $0.1 \%$ Tween (PBS-T), membranes were incubated for 1 hour at room temperature with the following secondary antibodies in blocking buffer: 1:5000 goat anti-rabbit IRDye 800 (Li-Cor \#926-32211) and 1:5000 donkey anti-mouse IRDye 680 (Li-Cor \#926-32222). Membranes were washed in PBS$\mathrm{T}$ and fluorescent bands were visualized using an Odyssey Infrared Imaging System (LiCor). Intensities of specific bands were quantified using ImageJ (http://rsbweb.nih.gov/ij/), corrected for background signal and GAPDH signal.

Within the BDNF blot, different BDNF domains were analyzed, distinguishing between mBDNF (14 kDa) and proBDNF (16 kDa), as previously described (Pang, et al., 2004; 
Sierksma, et al., 2012). The PDE5 antibody reportedly recognizes the two isoforms of PDE5A1 (99kDa) and PDE5A2 (89kDa), yet only the 99kDa was detectable in our blots.

\section{STATISTICAL ANALYSIS}

The basic readout parameter of the ORT is the time spent exploring each object, trials in which animals had explored both objects for less than 6 s in T1 or 9 s in T2 were excluded from analysis, since these data may not be reliable (Sven Akkerman, et al., 2012). We examined the effects of retention delay on exploration (e1 and e2) by performing a two-way ANOVA (Interval $x$ Housing) on all the vehicle treated conditions. The effect of the vardenafil treatment on exploration was also investigated using two-way ANOVA (Treatment $x$ Housing), though only in the $24 \mathrm{~h}$ interval. In the $48 \mathrm{~h}$ interval, the effects of vardenafil treatment were examined using one-way ANOVAs within each separate housing condition, because different doses were tested in each housing condition at the $48 \mathrm{~h}$ retention interval. Significant differences were further investigated using Tukey HSD post-hoc tests.

The effects of retention delay on object discrimination performance $(d 2)$ were examined using a two way ANOVA (Interval $x$ Housing) on all vehicle treated conditions. Housing effects on $d 2$ were further evaluated for each retention interval separately, using one-way ANOVA followed by Tukey HSD post-hoc tests. Effects of vardenafil on object discrimination were assessed by performing one-way ANOVA's on each housing condition separately, within the $24 \mathrm{~h}$ and $48 \mathrm{~h}$ retention intervals. Significant effects were further investigated using Tukey HSD post-hoc tests, comparing each different dose with the vehicle condition. In order to examine whether the animals actually showed object discrimination, the $d 2$ of each individual condition was also compared to 0 using one-sample $t$-tests.

Differences in hippocampal weight, stereological- and biochemical measures were also analyzed using a one-way ANOVA and post-hoc Tukey HSD tests. 


\section{RESULTS}

\section{OBJECT RECOGNITION TASK: EFFECTS OF HOUSING ON}

\section{EXPLORATION}

The exploration data of the animals is shown in Table 1. A two-way ANOVA (Interval $x$ Housing) was performed, incorporating only vehicle treated groups. Significant main effects were found for both Interval and Housing on e1 $\left(F^{\prime} s(2,161)>10.17, p^{\prime} s<\right.$ $0.001)$ and e2 $\left(F^{\prime} \mathrm{s}(2,161)=17.94, p^{\prime} \mathrm{s}<0.001\right)$, as well as significant Interval by Housing interaction effects $\left(F^{\prime} s(4,161)>16.16\right.$, $\left.p^{\prime} s<0.001\right)$. Therefore, one-way ANOVAs were performed on each separate retention interval.

Table 1 Exploration levels in the ORT

\begin{tabular}{|c|c|c|c|c|c|c|}
\hline Retention interval & $\begin{array}{l}\text { Vardenafil } \\
(\mathrm{mg} / \mathrm{kg})\end{array}$ & Dose & Housing & $\mathbf{n}$ & e1 & e2 \\
\hline \multirow[t]{3}{*}{$1 \mathrm{~h}$ Interval } & 0 & & SOL & 21 & $15.34(1.51)$ & $19.01(1.30)$ \\
\hline & 0 & & SOC & 17 & $43.48(3.04)$ & $52.08(4.49)$ \\
\hline & 0 & & $\mathrm{EE}$ & 18 & $40.10(4.91)$ & $46.55(5.14)$ \\
\hline \multirow[t]{12}{*}{24 h Interval } & 0 & & SOL & 22 & $29.88(1.87)$ & $28.45(2.01)$ \\
\hline & 0 & & SOC & 18 & $29.45(2.44)$ & $30.65(2.92)$ \\
\hline & 0 & & $\mathrm{EE}$ & 18 & $19.05(1.80)$ & $16.28(1.42)$ \\
\hline & 0.1 & & SOL & 22 & $29.91(2.27)$ & $27.24(1.49)$ \\
\hline & 0.1 & & SOC & 18 & $30.65(2.85)$ & $27.43(2.31)$ \\
\hline & 0.1 & & $\mathrm{EE}$ & 17 & $23.00(1.93)$ & $22.11(2.34)$ \\
\hline & 0.3 & & SOL & 22 & $29.23(2.15)$ & $25.83(1.59)$ \\
\hline & 0.3 & & SOC & 18 & $32.28(3.38)$ & $31.45(3.15)$ \\
\hline & 0.3 & & $\mathrm{EE}$ & 18 & $21.79(3.29)$ & $24.65(2.27)$ \\
\hline & 1 & & SOL & 22 & $29.96(2.26)$ & $31.74(2.36)$ \\
\hline & 1 & & SOC & 18 & $30.43(3.15)$ & $36.26(3.75)$ \\
\hline & 1 & & $\mathrm{EE}$ & 18 & $21.56(2.03)$ & $20.77(2.32)$ \\
\hline \multirow[t]{9}{*}{$48 \mathrm{~h}$ Interval } & 0 & & SOL & 21 & $24.02(1.53)$ & $28.66(1.72)$ \\
\hline & 0 & & SOC & 18 & $23.15(1.06)$ & $33.82(2.07)$ \\
\hline & 0 & & $\mathrm{EE}$ & 17 & $23.52(2.56)$ & $30.38(3.04)$ \\
\hline & 0.03 & & $\mathrm{EE}$ & 16 & $20.24(1.16)$ & $17.20(1.33)$ \\
\hline & 0.1 & & $\mathrm{EE}$ & 17 & $19.12(1.49)$ & $20.13(1.48)$ \\
\hline & 0.3 & & SOC & 18 & $24.69(2.25)$ & $36.84(2.01)$ \\
\hline & 0.3 & & $\mathrm{EE}$ & 17 & $25.51(3.27)$ & $24.12(2.76)$ \\
\hline & 1 & & SOL & 22 & $30.05(1.84)$ & $30.92(1.77)$ \\
\hline & 1 & & $\mathrm{EE}$ & 15 & $26.17(2.23)$ & $26.28(2.97)$ \\
\hline
\end{tabular}

This table shows the number of animals $(n)$ and mean exploration time $( \pm S E M)$ in seconds on both the objects in T1 (e1) and T2 (e2) in every treatment condition. Solitary (SOL), socially (SOC) and enriched (EE) housed animals were tested repeatedly, so every individual animal performed each experimental condition once. First the $1 \mathrm{~h}$ retention interval was tested, followed by the $24 \mathrm{~h}$ interval conditions and finally the 48 $\mathrm{h}$ retention. Of note, although $22 \mathrm{SOL}, 18 \mathrm{SOC}$ and $18 \mathrm{EE}$ animals were tested in each condition, animals that explored less than $6 s$ in T1 and/or less than $9 s$ in T2 were excluded from the analysis. 
At the $1 \mathrm{~h}$ interval, one-way ANOVA revealed significant differences were between vehicle treated housing conditions on e1 $(F(2,53)=22.59, p<0.001)$ and e2 $(F(2,53)$ $=23.36, p<0.001)$. Tukey HSD post-hoc tests revealed that SOL animals had a lower $\mathrm{e} 1$ and $\mathrm{e} 2$ compared to the SOC and EE animals. At the $24 \mathrm{~h}$ retention interval oneway ANOVA also revealed significant differences on e1 $(F(2,55)=8.72, p<0.01)$ and e2 $(F(2,55)=18.85, p<0.001)$ between the vehicle treated animals. Post-hoc analysis revealed that exploration levels ( $\mathrm{e} 1$ and $\mathrm{e} 2$ ) were lower in vehicle treated EE animals as compared to the two other housing conditions. At the $48 \mathrm{~h}$ retention interval, no significant differences between the housing conditions were found $\left(F^{\prime} s(2,53)<1.38\right.$, n.s.).

\section{OBJECT RECOGNITION TASK: EFFECTS OF VARDENAFIL TREATMENT ON EXPLORATION}

A Housing $x$ Treatment ANOVA was performed to investigate the effects vardenafil treatment on exploration levels in the $24 \mathrm{~h}$ retention interval. Because differed doses of vardenafil were tested in each housing condition on the $48 \mathrm{~h}$ interval, one-way ANOVA was used to analyze their effects within each housing condition separately.

\section{H RETENTION INTERVAL}

Housing $x$ Treatment ANOVA at the $24 \mathrm{~h}$ retention interval showed that there were no overall Treatment effects on e1 or e2 $(F(3,219)=0.31$ and $F(3,219)=2.18$, both n.s.). There were, on the other hand, significant overall effects of Housing on exploration $(e 1: F(2,219)=16.31$, e2: $F(2,219)=19.39$, both $p<0.001)$ and Treatment by Housing interaction effects $\left(F^{\prime} s(6,219)<1.71\right.$, n.s.). Post-hoc analysis with Tukey HSD tests showed that, on the $24 \mathrm{~h}$ retention interval, the overall exploration (both e1 and e2) of EE animals was lower compared to SOL and SOC animals. 


\section{H RETENTION INTERVAL}

In the SOL animals, one-way ANOVA showed that animals treated with $1 \mathrm{mg} / \mathrm{kg}$ vardenafil had a higher e1 compared to vehicle treated animals $(F(1,41)=6.30, p<$ $0.05)$ but that no differences were present e2 $(F(1,41)=0.84$, n.s.). In the $E E$ animals, no effect was found of Treatment on e1 $(F(4,77)=1.85$, n.s. $)$, however, there was an effect of Treatment on e2 $(F(4,77)=4.54, p<0.01)$. Post-hoc analysis showed that $\mathrm{EE}$ animals treated with 0.03 and $0.1 \mathrm{mg} / \mathrm{kg}$ vardenafil had a lower e2 compared to vehicle treated EE animals. Finally, in SOC animals, no effects of vardenafil treatment on exploration levels were found ( $F^{\prime} s<1.10$, n.s.).

\section{OBJECT RECOGNITION TASK: EFFECTS OF RETENTION DELAY ON OBJECT DISCRIMINATION}

The discrimination performance of the animals is shown in Figures 2-4. A two-way ANOVA (Interval x Housing) was performed, incorporating only vehicle treated groups. There was a significant main effect of Interval on discrimination performance $(F(2,161)$ $=21.33, p<0.001)$. Post-hoc analysis revealed that the overall $d 2$ value was higher at the $1 \mathrm{~h}$ interval compared to the $24 \mathrm{~h}$ and $48 \mathrm{~h}$ intervals. There was also a significant main effect of Housing on $d 2(F(2,161)=3.05, p<0.05)$. Post-hoc analysis revealed that EE animals showed better overall object discrimination ( $d 2$ was higher) compared to SOC animals. No significant Interval by Housing interaction effects were found for $d 2(F(4,161)=0.50$, n.s $)$.

One-way ANOVA's were performed on the vehicle conditions of each separate retention interval. No significant $d 2$ differences were found between the housing conditions at the $1 \mathrm{~h}(F(2,53)=0.19$, n.s. $)$ and $48 \mathrm{~h}(F(2,53)<0.63$, n.s. $)$ retention intervals. However, there were significant differences between the $d 2$ measures at the $24 \mathrm{~h}$ interval $(F(2,55)=3.49, p<0.05)$. Post-hoc analysis revealed that the $d 2$ value of EE animals was higher at the $24 \mathrm{~h}$ retention interval, compared to SOC animals. 
One-sample $t$-tests on the vehicle treated conditions showed that, at the $1 \mathrm{~h}$ retention interval, the $d 2$ measure was significantly higher than zero in all vehicle treated housing conditions. Yet, after the $24 \mathrm{~h}$ retention interval, only EE animals still discriminated between the novel and familiar objects. After the $48 \mathrm{~h}$ retention interval, none of the groups still showed significant object discrimination. The effects of retention delay on the different housing conditions are graphically presented in Figure 2.

\section{ORT - Delay dependent forgetting}

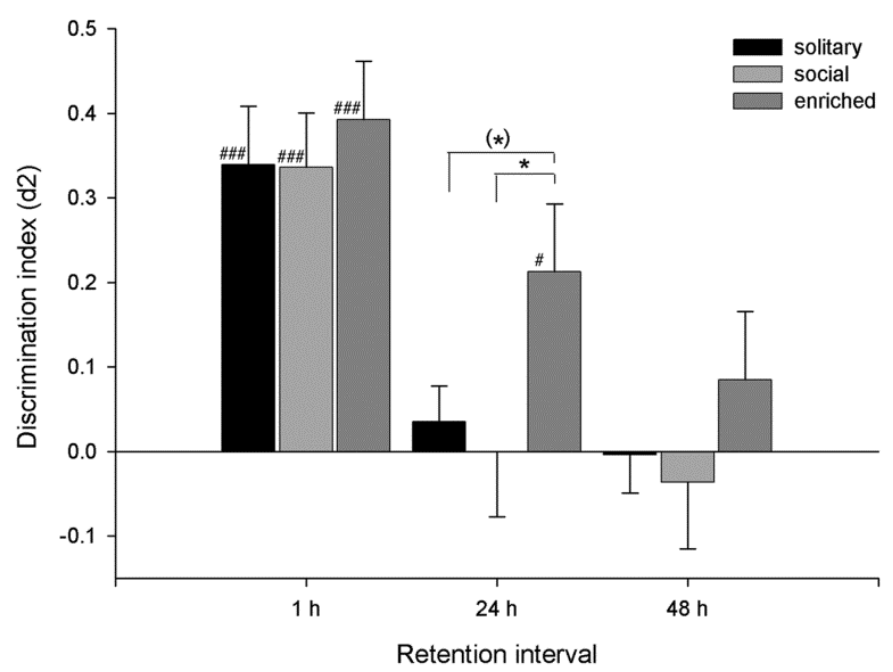

Figure 2 Effect of retention delay on object discrimination

Discrimination performance $(d 2$, mean $+S E M)$ of SOL, SOC and EE rats treated with vehicle in a $1 \mathrm{~h}$, and 24 $\mathrm{h}$ and $48 \mathrm{~h}$ retention interval ( $\mathrm{x}$-axis) in the object recognition task. A difference from zero is indicated with hash symbols (one-sample t-test; \#: $p<0.05$; \#\#\#: $p<0.001$ ), differences between conditions within the same retention interval are indicated with asterisks (Tukey HSD; $\left({ }^{*}\right): p<0.10 ; *: p<0.05$ ).

\section{OBJECT RECOGNITION TASK: EFFECTS OF VARDENAFIL ON OBJECT DISCRIMINATION}

Because there are differences in doses used in the $24 \mathrm{~h}$ and $48 \mathrm{~h}$ retention intervals, the effects of vardenafil were analyzed separately, using one-way ANOVAs for each housing condition/retention interval. Of note, the vehicle groups used in this section 
are the same as those in the section 'Object recognition task: effects of retention delay on object discrimination'.

\section{H RETENTION INTERVAL}

In the $24 \mathrm{~h}$ interval, one-way ANOVAs within each separate Housing condition revealed no significant differences between $d 2$ values of the different vardenafil treatments $\left(F^{\prime} S<0.34\right)$, with the exception of the SOL animals $(F(3,84)=4.35, p<$ 0.01 ). Post-hoc analysis showed that the $d 2$ of SOL animals treated with $1 \mathrm{mg} / \mathrm{kg}$ vardenafil was increased compared to vehicle treated animals.

One-sample $t$-tests showed that SOL animals treated with 0.3 or $1 \mathrm{mg} / \mathrm{kg}$ vardenafil had a $d 2$ value significantly higher than zero. The same was true for SOC animals treated with $0.3 \mathrm{mg} / \mathrm{kg}$ vardenafil. EE animals had a $\mathrm{d} 2$ that was higher than zero at all the tested vardenafil doses, including the vehicle condition. The effects of vardenafil in the $24 \mathrm{~h}$ retention interval are presented in Figure 3.

ORT - 24h interval - solitary animals

ORT - $24 \mathrm{~h}$ interval - social animals

ORT - $24 \mathrm{~h}$ interval - enriched animals

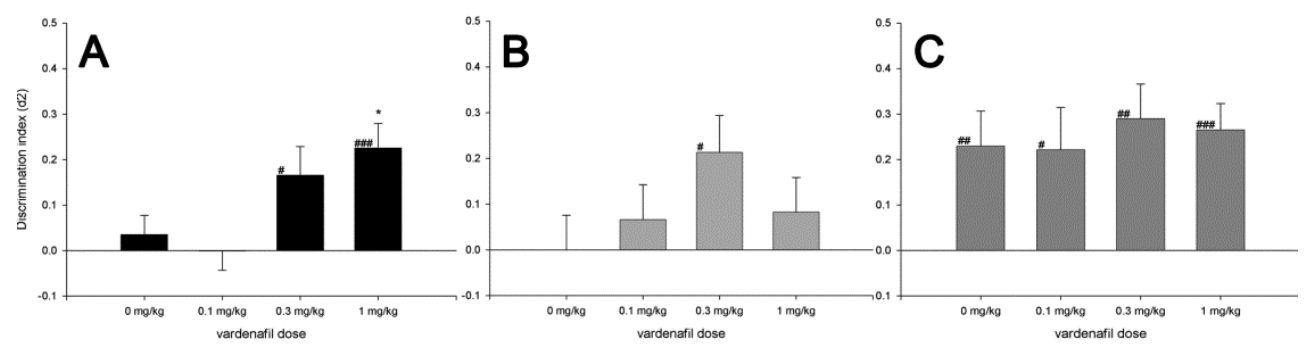

Figure 3 ORT $24 \mathrm{~h}$ intervals

Discrimination performance (d2, mean + SEM) of SOL (A), SOC (B) and EE (C) rats after administration of different doses of vardenafil in the object recognition task. All housing conditions were tested in a $24 \mathrm{~h}$ retention interval in combination with $0,0.1,0.3$ and $1 \mathrm{mg} / \mathrm{kg}$ vardenafil ( $x$-axis). A difference from zero is indicated with hash symbols (one-sample t-test; \#: $p<0.05$; \#\#: $p<0.01$; \#\#\#: $p<0.001$ ), differences from the vehicle condition $(0 \mathrm{mg} / \mathrm{kg}$ ) within the same retention interval are indicated with an asterisk (Tukey HSD post-hoc tests, $\left.{ }^{*}: p<0.05\right)$. Of note, the $0 \mathrm{mg} / \mathrm{kg}$ vardenafil conditions are identical to those used in Figure2.

\section{H RETENTION INTERVAL}

In the $48 \mathrm{~h}$ retention interval, only the most effective dose from the $24 \mathrm{~h}$ interval was tested in the SOL and SOC animals. We analyzed the treatment effects of vardenafil treatment within each housing condition using one-way ANOVA, revealing an effect 
of Treatment on the discrimination $(d 2)$ performance of the SOL animals $(F(1,41)=$ 12.13, $p<0.001)$, and the SOC animals $(F(1,34)=7.92, p<0.01)$. This means that after $48 \mathrm{~h}$ hours, object discrimination was still improved by the vardenafil doses of 1 $\mathrm{mg} / \mathrm{kg}$ and $0.3 \mathrm{mg} / \mathrm{kg}$ in SOL and SOC animals, respectively. However, in EE animals, one-way ANOVA revealed no significant Treatment effect on discrimination performance $(d 2)(F(4,77)=0.58$, n.s. $)$.

One-sample $t$-tests showed that the $d 2$ of the SOL and SOC differed from zero at a dose of $1 \mathrm{mg} / \mathrm{kg}$ and $0.3 \mathrm{mg} / \mathrm{kg}$ vardenafil, respectively. However, none of the tested vardenafil doses improved the discrimination performance of the EE animals to a level above zero at the $48 \mathrm{~h}$ retention interval. The discrimination performances of the housing conditions at the $48 \mathrm{~h}$ retention interval are graphically presented in Figure 4.

ORT - 48h interval - solitary animals

ORT - $48 \mathrm{~h}$ interval - social animals

ORT - 48h interval - enriched animals

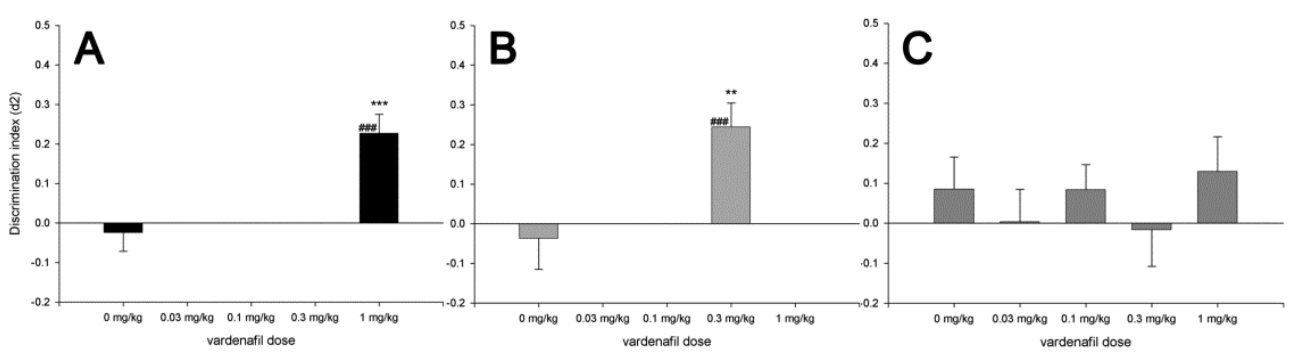

\section{Figure 4 ORT $48 \mathrm{~h}$ intervals}

Discrimination performance $(d 2$, mean + SEM) of SOL $(\mathbf{A})$, SOC $(\mathbf{B})$ and EE $(\mathbf{C})$ rats after administration of different doses of vardenafil in the object recognition task. All housing conditions were tested in a $48 \mathrm{~h}$ retention interval in combination with $0,0.1,0.3$ and $1 \mathrm{mg} / \mathrm{kg}$ vardenafil (x-axis), EE animals also received $0.03 \mathrm{mg} / \mathrm{kg}$ vardenafil. A difference from zero is indicated with hash symbols (one-sample $t$-test; \#\#\#: $p<$ $0.001)$, differences from the vehicle condition $(0 \mathrm{mg} / \mathrm{kg})$ within the same retention interval are indicated with asterisks (Tukey HSD, $* *: p<0.01 ; * * *: p<0.001)$. Of note, the $0 \mathrm{mg} / \mathrm{kg}$ vardenafil conditions are identical to those used in Figure 2.

\section{DORSAL HIPPOCAMPAL WEIGHT}

One-way ANOVA revealed significant differences in dorsal hippocampal weights between the different housing conditions $(F(2,26)=4.36, p=0.023)$. Post-hoc analysis revealed that EE animals had a heavier dorsal hippocampus, though only compared to SOC animals. However, there was also a strong trend ( $p=0.052)$ 
towards a significant difference between EE and SOL animals (Table 2/Figure 5).

Dorsal hippocampal weight

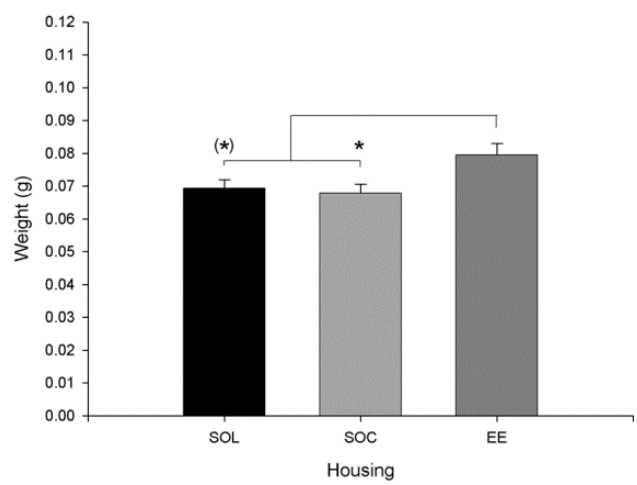

Figure 5 Dorsal hippocampal weight

Dorsal hippocampal weights of the three experimental groups in grams (mean + SEM). Significant differences between groups are indicated with asterisks (Tukey HSD post-hoc tests $\left({ }^{*}\right): p=0.052 ;{ }^{*}: p<$ 0.05).

\section{STEREOLOGY}

The DG, CA1 and CA2-3 of the dorsal hippocampus were compared between the different housing conditions (Table $2 /$ Figure 6). One-way ANOVA revealed no differences on total cell numbers $\left(F^{\prime} s(2,12)<1.50\right.$, n.s. $)$ and cell density $\left(F^{\prime} s(2,12)<\right.$ 2.13 , n.s.). The only significant difference found was on the volume of the DG region $(F(2,12)=19,26, p<0.001)$ which was larger in SOL animals, compared to both other groups.

Volume

Total cell number

Cell density
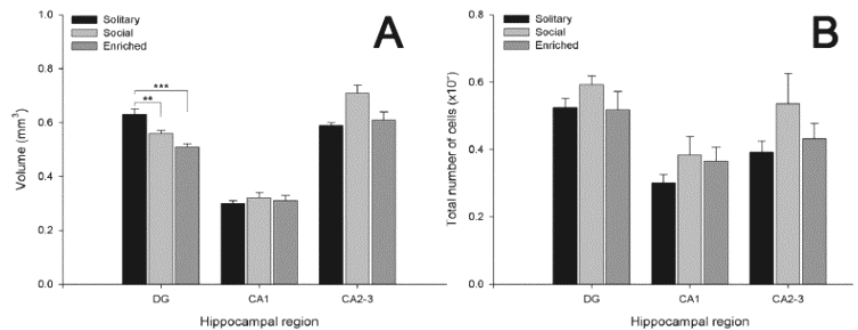

B

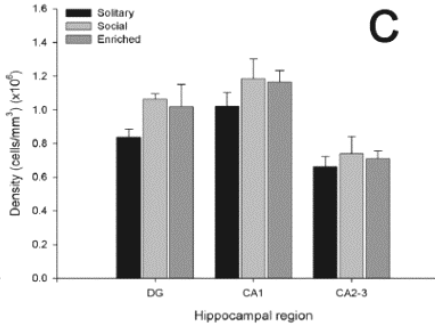

Figure 6 Stereology

The volume (mm3) total number of cells and cell density (number of cells/mm3) in the DG, CA1 and CA2-3 sub-regions of dorsal hippocampus of solitary-, socially- and enriched housed rats. One-way ANOVA only revealed significant differences in the DG, significant differences are indicated with asterisks (Tukey HSD, $\left.* *: p<0.01 ;{ }^{* *}: p<0.001\right)$. 


\section{PDE5 MRNA AND PROTEIN EXPRESSION}

One-way ANOVA revealed no differences in PDE5 mRNA expression between the housing conditions in the dorsal hippocampus $(F(2,24)=1.41$, n.s.; Table $2 /$ Figure $7 A)$. In line with mRNA expression levels, there were also no differences detected between PDE5A1 protein levels between the different housing conditions $(F(2,14)=$ 0.21 , n.s.; Table 2/ Figure 7B).

PDE5 MRNA

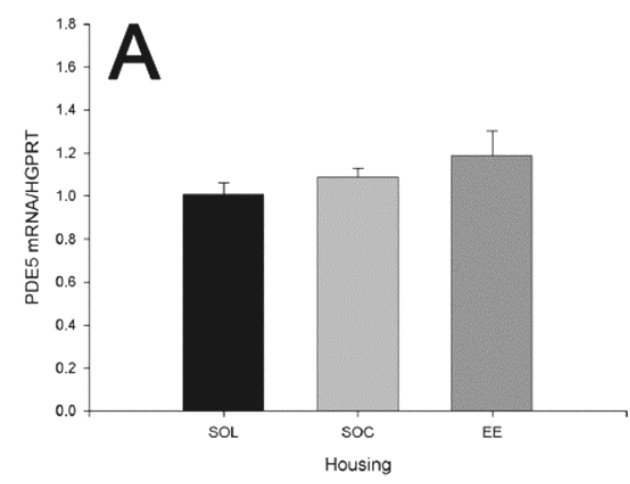

PDE5

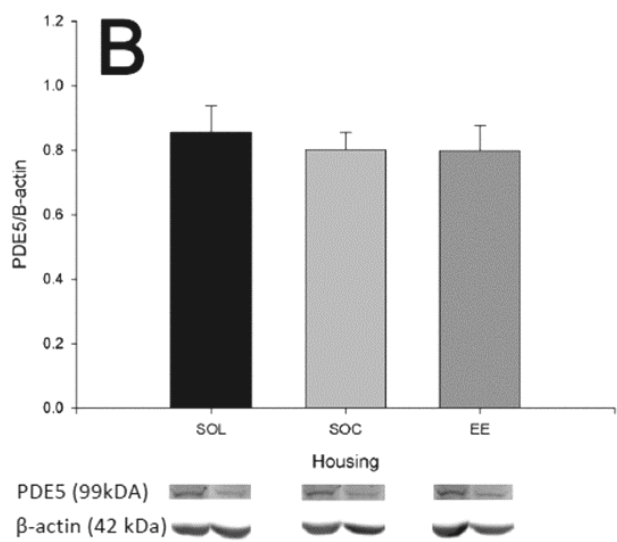

Figure 7 PDE5 mRNA and protein concentration in the dorsal hippocampus

(A) qPCR analysis showing mRNA levels of PDE5 of the three experimental conditions. Data was normalized relative to the housekeeping gene HGPRT. Analysis with one-way ANOVA revealed no significant differences between groups. (B) Western Blot analysis showing relative protein expression of PDE5, corrected for $\beta$-actin, of the three experimental groups in the dorsal hippocampus. Analysis with one-way ANOVA revealed no significant differences between groups. Bars indicate mean + SEM per group.

\section{Western BLOTTING}

Differences between protein levels of the different housing condition in the dorsal hippocampus were investigated using Western Blots (Table 2). One-way ANOVA between the housing conditions revealed no differences in the levels of synaptophysin $(F(2,22)=0.74$, n.s.; Figure $8 \mathrm{~A})$, PSD95 $(F(2,26)=1.99$, n.s.; Figure $8 \mathrm{~B})$, proBDNF $(F$ $(2,25)=0.72$, n.s.; Figure $9 A)$, mature $\operatorname{BDNF}(F(2,24)=1.78$, n.s.; Figure 9B), 
Synaptophysin

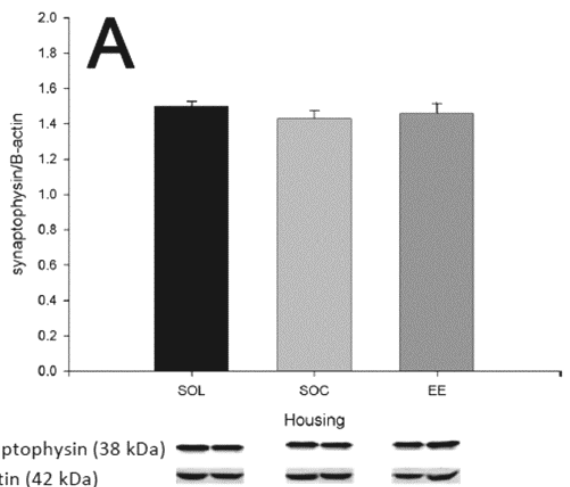

PSD95

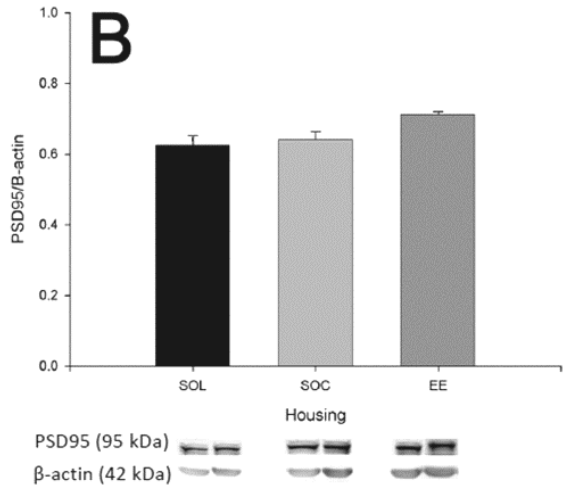

Figure 8 Protein levels of synaptophysin and PSD95 in the dorsal hippocampus

Western Blot analysis showing protein levels of synaptophysin (A) and PSD95 (B) of the three experimental conditions. Data was normalized relative to the housekeeping protein $\beta$-actin. Bars indicate mean + SEM per group. One-way ANOVA revealed no statistical differences between the experimental groups.

proBDNF

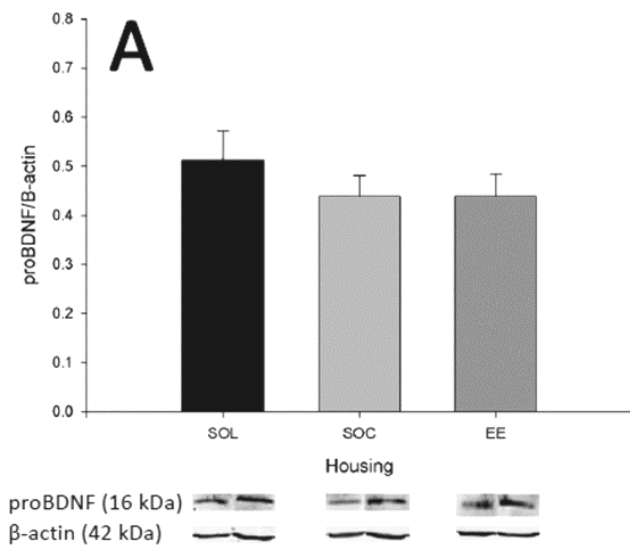

mBDNF

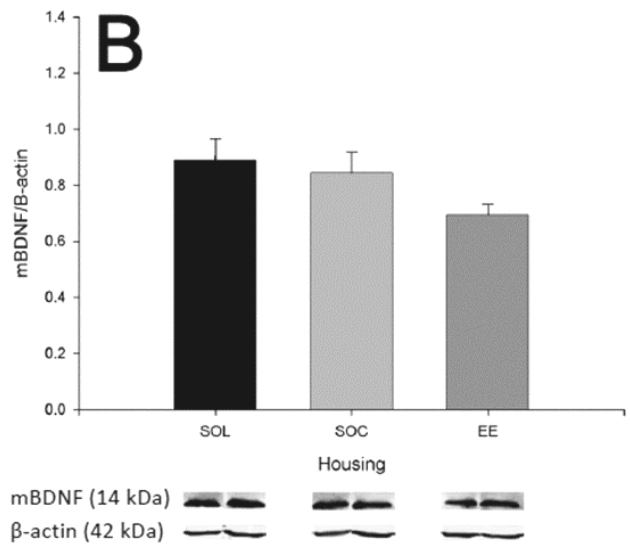

Figure 9 Protein levels of proBDNF and mBDNF in the dorsal hippocampus

Western Blot analysis showing protein levels of proBDNF (A) and mBDNF (B) of the three experimental conditions. Data was normalized relative to the housekeeping protein $\beta$-actin. Bars indicate mean + SEM per group. One-way ANOVA revealed statistical differences were observed between the experimental groups. 

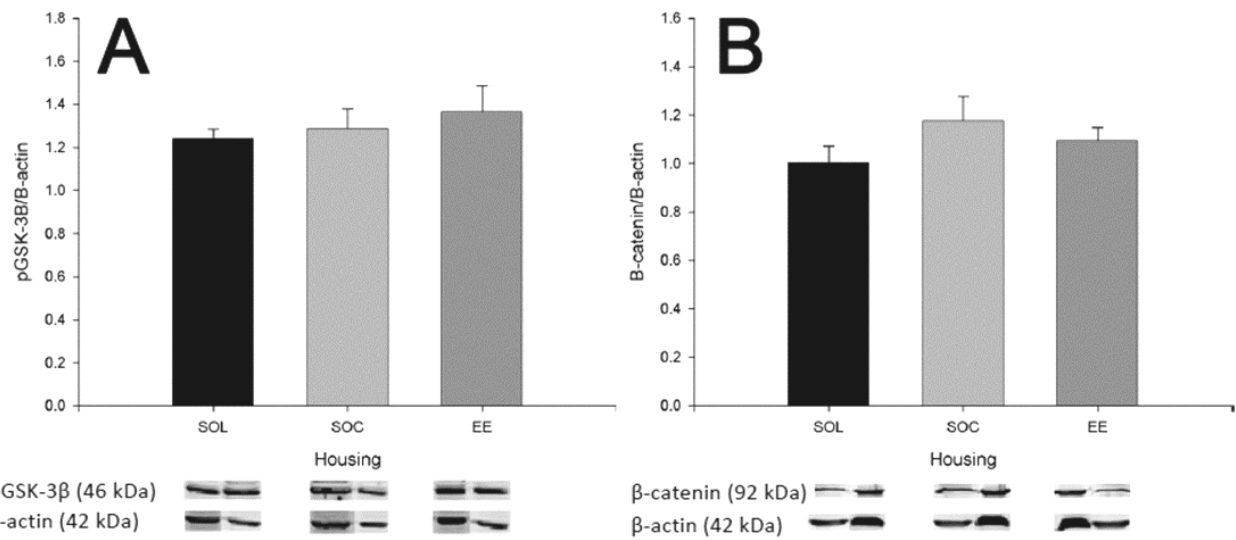

Figure 10 Protein levels of pGSK-3 $\beta$ and $\beta$-catenin in the dorsal hippocampus

Western Blot analysis showing protein levels of $\operatorname{pGSK}-3 \beta(A)$, and $\beta$-catenin (B) of the three experimental groups. Data was normalized relative to the housekeeping protein $\beta$-actin. Bars indicate mean + SEM per group. SOL: solitary housed animals, SOC: socially housed animals, EE: animals housed in an enriched environment. Data was analyzed using a one-way ANOVA, no statistical differences were observed between the experimental groups.

ERK2
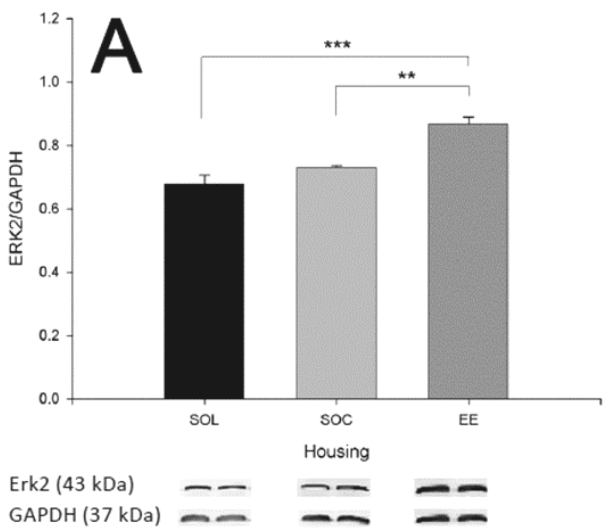

PCREB

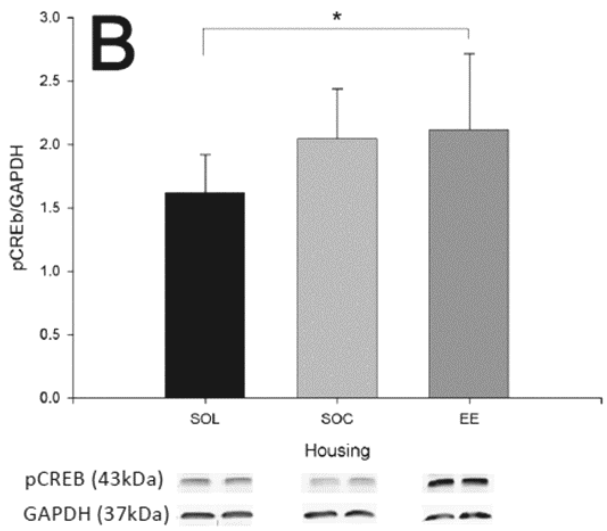

Figure 11 Protein levels of ERK2 and PCREB in the dorsal hippocampus

Western Blot analysis showing protein levels of ERK2 (A) and PCREB $\left(B_{i}\right)$ of the three experimental conditions. Data was normalized relative to the housekeeping protein GAPDH. Bars indicate mean + SEM per group. SOL: solitary housed animals, SOC: socially housed animals, EE: animals housed in an enriched environment. Data was analyzed using Tukey HSD post-hoc tests $(*$ : $p<0.05 ; * *$ : $p<0.01 ; * * *$ : $p<0.001$ ). 
pGSK-3 $\beta(F(2,22)=0.46$, n.s.; Figure $10 \mathrm{~A})$ and $\beta$-catenin $(F(2,24)=1.32$, n.s.; Figure $10 \mathrm{~B})$. On the other hand, there were significant differences in the protein levels of $\operatorname{ERK2}(F(2,24)=17.62, p<0.001$; Figure 11A) and pCREB $(F(2,26)=3.79, p<0.05$; Figure 11B). Post-hoc analysis showed that ERK2 was increased in EE animals compared to SOL and SOC animals and the expression of pCREB was increased in EE animals, compared to SOL animals.

Table 2 Results stereological and biochemical measurements

\begin{tabular}{|c|c|c|c|c|c|}
\hline Housing Condition & Figure & & SOL & SOC & EE \\
\hline Weight dorsal hippocampus (mg) & Figure 5 & & $69.3(2.60)(*)$ & $67.9(2.60)+$ & $72.0(3.60)(*) /+$ \\
\hline \multirow{3}{*}{$\begin{array}{l}\text { Volume dorsal hippocampus } \\
\text { (mm3) }\end{array}$} & Figure $6 \mathrm{~A}$ & $\overline{D G}$ & $0.63(0.02)^{\star \star *} / \# \#$ & $0.56(0.01) \# \#$ & $0.51(0.01)^{* * *}$ \\
\hline & & CA1 & $0.30(0.01)$ & $0.32(0.02)$ & $0.31(0.02)$ \\
\hline & & CA3 & $0.59(0.01)$ & $0.71(0.03)$ & $0.61(0.03)$ \\
\hline \multirow{3}{*}{$\begin{array}{l}\text { Cells dorsal hippocampus } \\
\left(\times 10^{6}\right)\end{array}$} & Figure $6 \mathrm{~B}$ & $\overline{D G}$ & $0.53(0.03)$ & $0.59(0.02)$ & $0.52(0.06)$ \\
\hline & & CA1 & $0.30(0.02)$ & $0.38(0.05)$ & $0.37(0.04)$ \\
\hline & & CA3 & $0.39(0.03)$ & $0.54(0.09)$ & $0.43(0.04)$ \\
\hline \multirow{3}{*}{$\begin{array}{l}\text { Density dorsal hippocampus } \\
\left(\times 10^{6} / \mathrm{mm} 3\right)\end{array}$} & Figure $6 \mathrm{C}$ & $\overline{D G}$ & $0.84(0.05)$ & $1.06(0.03)$ & $1.02(0.13)$ \\
\hline & & CA1 & $1.02(0.08)$ & $1.19(0.12)$ & $1.16(0.07)$ \\
\hline & & CA3 & $0.66(0.06)$ & $0.74(0.10)$ & $0.71(0.05)$ \\
\hline PDE5 mRNA (PDE5 mRNA/HGPRT) & Figure 7A & & $1.00(0.06)$ & $1.09(0.04)$ & $1.19(0.11)$ \\
\hline PDE5 protein (PDE5/ß-actin) & Figure 7B & & $0.86(0.08)$ & $0.80(0.05)$ & $0.80(0.08)$ \\
\hline Synaptophysin (Synaptophysin//-actin) & Figure $8 \mathrm{~A}$ & & $1.50(0.03)$ & $1.43(0.04)$ & $1.43(0.06)$ \\
\hline PSD95 (PSD95/ß-actin) & Figure $8 \mathrm{~B}$ & & $0.62(0.30)$ & $0.64(0.02)$ & $0.71(0.01)$ \\
\hline proBDNF (proBDNF/ $\beta$-actin) & Figure $9 \mathrm{~A}$ & & $0.51(0.06)$ & $0.44(0.04)$ & $0.44(0.05)$ \\
\hline mBDNF (mBDNF/B-actin) & Figure 9B & & $0.89(0.08)$ & $0.84(0.07)$ & $0.69(0.04)$ \\
\hline 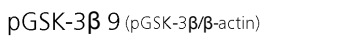 & Figure $10 \mathrm{~A}$ & & $1.24(0.04)$ & $1.29(0.09)$ & $1.37(0.12)$ \\
\hline$\beta$-catenin ( $\beta$-catenin $\beta$-actin) & Figure 10B & & $1.00(0.07)$ & $1.18(0.10)$ & $1.10(0.05)$ \\
\hline ERK2 (ERK2/GAPDH) & Figure $11 \mathrm{~A}$ & & $0.68(0.03)^{* * *}$ & $0.73(0.01)++$ & $0.87(0.02)^{* * * /++}$ \\
\hline$p C R E B$ (pCREB/GAPDH) & Figure 11B & & $1.62(0.09)^{*}$ & $2.04(0.13)$ & $2.11(0.20)^{*}$ \\
\hline
\end{tabular}

Comparisons of the stereological and biochemical measurements between the different housing conditions using one-way ANOVA and Tukey HSD post hoc analysis. Individual cells show the mean (+SEM) of each housing condition. Significant differences between $\mathrm{SOL}$ and EE animals are indicated by asterisks $(*): p=$ $\left.0.052 ; *: p<0.05 ;{ }^{* *}: p<0.001\right)$, significant differences between SOL and SOC animals are indicated by hash signs (\#\#: $p<0.01)$ and significant differences between $S O C$ and EE animals are indicated by plus signs $(+: p<0.05 ;++: p<0.01)$.

\section{DISCUSSION}

By analyzing the vehicle conditions, we examined whether housing conditions affected baseline memory performance of rats in an ORT. One hour after learning, object memory appeared to be equal for all housing groups, as all groups discriminated equally well between the novel and familiar objects. However, after 24 h EE animals outperformed both other groups, which no longer showed any sign of object memory after this retention interval. These findings are in accordance with 
previous studies (Bechara \& Kelly, 2013; Bruel-Jungerman, et al., 2005; Pamplona, Pandolfo, Savoldi, Prediger, \& Takahashi, 2009; Simpson \& Kelly, 2011, 2012) that demonstrate that EE enhances cognitive performance. After $48 \mathrm{~h}$, none of the housing conditions discriminated between objects anymore, thereby indicating that also the EE animals had also forgotten the object information at this retention interval.

Multiple studies have produced evidence which suggests that vardenafil has memory enhancing properties in SOL and SOC rats (Akkerman, Blokland, \& Prickaerts, 2014; Devan, et al., 2004; Prickaerts, et al., 2004; Prickaerts, van Staveren, et al., 2002; Reneerkens, et al., 2012; Rutten, et al., 2007; Rutten, et al., 2009; van Donkelaar, et al., 2008). The main goal of the current study was to investigate whether these memory enhancing effects could be extended to EE rats, as it is known that EE promotes behavior that is more similar to the animal's natural behavior (Hutchinson, Avery, \& Vandewoude, 2005; Viola, et al., 2010) and may therefore more closely resemble human living conditions (Gurwitz, 2001). As in previous studies (Akkerman, et al., 2014; Prickaerts, van Staveren, et al., 2002; Rutten, et al., 2007; Rutten, et al., 2009), object memory of SOL animals was effectively enhanced by $1 \mathrm{mg} / \mathrm{kg}$ vardenafil in a $24 \mathrm{~h}$ retention interval. SOL animals also showed intermediate memory improvement at a dose of $0.3 \mathrm{mg} / \mathrm{kg}$, as performance was above chance level but not improved compared to vehicle-treatment (Akkerman, Prickaerts, et al., 2012). In SOC animals, an intermediate vardenafil effect was also found with a $0.3 \mathrm{mg} / \mathrm{kg}$ dose, yet $1 \mathrm{mg} / \mathrm{kg}$ was ineffective. Vehicle treated EE animals still showed object memory after $24 \mathrm{~h}$, and none of the tested vardenafil doses were able to improve memory performance at this interval. Thus, vardenafil was ineffective in improving cognition in EE animals, asserted an intermediate effect in SOC animals, and successfully improved memory in SOL animals. Also, the most effective dose in SOC animals was lower and the effective dose-range was smaller compared to SOL animals, demonstrating that the stress of social isolation alone is enough to cause marked differences in the efficacy of vardenafil. The lack of effect in EE animals might be due to a ceiling effect, since the higher performance in the vehicle condition might leave less room for improvement by vardenafil. Hence, it was decided to extend the retention interval to $48 \mathrm{~h}$. 
After the $48 \mathrm{~h}$ retention interval, there was no more object discrimination in any of the housing conditions, when treated with vehicle. Even after $48 \mathrm{~h}$, memory performance in SOL $(1 \mathrm{mg} / \mathrm{kg})$ and SOC $(0.3 \mathrm{mg} / \mathrm{kg})$ animals was still fully enhanced by vardenafil. However, like in the $24 \mathrm{~h}$ retention interval, none of the tested vardenafil doses caused memory improvement in EE rats in the $48 \mathrm{~h}$ retention interval, ruling out the possibility of a ceiling effect. The lowered effective dose in SOC animals compared to SOL animals suggests that the effective dose might be even lower in $\mathrm{EE}$ animals and the lowest tested dose of $0.03 \mathrm{mg} / \mathrm{kg}$ may still have been too much. On the other hand, considering the low brain-plasma ratio and IC50 of vardenafil, it is questionable as to whether there would still be enough vardenafil available in the brain to be biologically effective with a further lowering of the dose (Reneerkens, et al., 2012). Alternatively, it could be argued that the effective dose range in EE animals was so narrow that the effect was simply missed. However, based on our previous data with PDE inhibitors in SOL animals, we assume that EE animals are insensitive to vardenafil treatment. This lack of effect of vardenafil on memory in $\mathrm{EE}$ rats resembles the ineffectiveness of this drug on human memory performance (Grass, et al., 2001; Reneerkens, et al., 2013; Schultheiss, et al., 2001) and suggests that, compared to SOL/SOC animals, the EE rat could be considered as a more valid animal model for testing cognition enhancing drugs (Gurwitz, 2001; Lynch \& Gall, 2013).

When animals display a statistically significant preference for the novel-objects, it can be safely inferred that they were able to remember/recognize the sample objects. On the other hand, if animals fail to display a preference for the novel object, this may not necessarily indicate an absence of object memory as perceptual, attentional and motivational mechanisms also play an important role in the manifestation of a preference for the novel object (Ennaceur, 2010; Gaskin, et al., 2010; Mumby, 2001). However, as all housing conditions showed a preference for the novel objects at the $1 \mathrm{~h}$ retention interval, we can assume that these processes were intact in the current study. In a minority of the test conditions, a housing condition or vardenafil treatment appeared to affect exploration levels. However, as previousy reported in other studies (Akkerman, Blokland, et al., 2012; Gaskin, et al., 2010), exploration levels were not related to object discrimination performance. PDE5-Is have shown performance 
enhancing effects across a wide variety of memory tasks, including; the Elevated Plus Maze (EPM) (Akar, Mutlu, Komsuoglu Celikyurt, et al., 2014), Radial Arm Maze (RAM), Morris Water Maze (MWM) (Akar, Mutlu, Celikyurt, et al., 2014; HosseiniSharifabad, et al., 2012). Y-maze (Jin, et al., 2014; Uthayathas, et al., 2013), Social Transmission Food Preference (STFP) task as well as active- (Jin, et al., 2014) and inhibitory avoidance tests (Akar, Mutlu, Komsuoglu Celikyurt, et al., 2014; Boccia, Blake, Krawczyk, \& Baratti, 2011). Therefore, we believe that the observed effects (or lack thereof) of vardenafil treatment on discrimination performance under the different housing conditions, are due to a success (or failure) to improve memory processes.

The insensitivity of EE animals to vardenafil treatment may be related to changes in brain plasticity, influencing their memory performance. To assess brain plasticity, we analyzed several morphological and neurochemical signaling markers. Based on the enhanced ORT performance and the increased dorsal hippocampal weight in the EE rats, we argue that an effective EE procedure was used in the present study. However, we did not find any effect of Housing in the dorsal hippocampus on absolute cell numbers nor on the synaptic markers synaptophysin and PSD95. Neither did we observe a change in pro- or mature BDNF levels, a neurotrophic protein typically implicated in plastic changes. This appears to be in contrast with the findings of most studies on EE (Cao, et al., 2014; Chourbaji, et al., 2012; Ickes, et al., 2000; RamirezRodriguez, et al., 2014; Simpson, Bree, \& Kelly, 2012; Simpson \& Kelly, 2011; Young, Lawlor, Leone, Dragunow, \& During, 1999). However, there are also EE studies that have reported cognitive improvements without any changes in hippocampal BDNF levels (Bindu, et al., 2007; Hicks, et al., 2002; Turner \& Lewis, 2003; Vedovelli, et al., 2011; Viola, et al., 2010), implicating that hippocampal BDNF is not essential for the observed cognition improvement found in EE animals. Of note, discrepancies between EE studies in morphological and biochemical effects can be caused by a variety of factors, such as; exercise level, strain, age, strain, EE protocols and differences in species of the animals involved in the studies (Viola \& Loss, 2014). 
We also assessed two BDNF-related signaling pathways, the MAP kinase/CREB signaling pathway and the AKT/B-catenin pathway. ERK is a kinase, activated by BDNF signaling, which phosphorylates the transcription factor CREB. CREB is involved in the expression of genes important for plasticity (e.g. neurogenesis or synaptogenesis) as well as memory processes (Vivar, Potter, \& van Praag, 2013). AKT is another kinase activated by BDNF signaling. Dephosphorylated GSK-3 $\beta$ is phosphorylated by AKT and inhibits several proteins (Grimes \& Jope, 2001) like $\beta$-catenin which, in its dephosphorylated state, translocates to the nucleus where it induces the transcription of specific genes involved in neurogenesis and synaptogenesis (Okuda, Yu, Cingolani, Kemler, \& Goda, 2007; Varela-Nallar \& Inestrosa, 2013). Thus, phosphorylation of GSK-3 $\beta$ leads to disinhibition of $\beta$-catenin, resulting in more neurogenesis or synaptogenesis. AKT/ $\beta$-catenin signaling appeared unaffected by EE as PGSK-3 $\beta$ and $\beta$-catenin levels were unchanged. Interestingly, MAP kinase/CREB signaling was increased as ERK expression and phosphorylation of CREB were enhanced in the dorsal hippocampus of EE rats. Yet, these increases are apparently not BDNF dependent.

The additional exercise in EE has been shown to be the major stimulant of BDNF increases and neurogenic effects (Bechara \& Kelly, 2013; Kobilo, Liu, et al., 2011; Kobilo, Yuan, \& van Praag, 2011), which are assumed to enhance memory performance (Bekinschtein, Oomen, Saksida, \& Bussey, 2011). However, it has recently been shown that $\mathrm{EE}$ in the absence of exercise can also improve object recognition memory, perhaps via mechanisms that are independent of BDNF upregulation and neurogenesis in the dentate gyrus of the hippocampus, as the latter two effects were not observed (Bechara \& Kelly, 2013). Consequently, we argue that in the present study, the level of exercise might not have been high enough to increase BDNF as well as cell numbers and probably also the number of synapses in our animals.

The hippocampus has been shown to contain PDE5 mRNA and protein, though at low levels and in different types of cells (see Reneerkens, et al., 2012). PDE5 mRNA and protein expression were assessed in the dorsal hippocampus. However, no differences 
were found between the different housing conditions on either of these measures. This indicates that the effects of EE on the efficacy of vardenafil cannot be explained by a difference in target enzyme availability.

It has been suggested that the cognition-enhancing effects of vardenafil are related to activation of cellular CGMP-protein kinase G (PKG)/CREB pathways implicated in glutamatergic signaling (Reneerkens, Rutten, Steinbusch, Blokland, \& Prickaerts, 2009; Rutten, et al., 2007). As described above, CREB activation is already enhanced in the dorsal hippocampus of EE rats. Therefore, a possible explanation could be hypothesized that vardenafil treatment did not further enhance CREB signaling since it was already fully stimulated in the hippocampus of EE rats.

Taken together, the present study showed that baseline memory performance was better in $\mathrm{EE}$ rats compared to $\mathrm{SOL}$ and $\mathrm{SOC}$ animals in object recognition task, i.e. they were able to remember the objects for a longer period. However, no effects were found in the dorsal hippocampus on BDNF levels, number of cells and markers for synapses. Furthermore, the effectiveness of vardenafil diminished with the level of enrichment, yet the target of vardenafil, PDE5, was unaffected by EE. The effect of EE on vardenafil efficacy is probably due to a more general mechanism which means that $\mathrm{EE}$ is also likely to affect other classes of drugs. If EE reduces the efficacy of drugs via widespread, general molecular memory mechanisms, this might partly explain the discrepancy in success rate between pre-clinical animal drug studies and studies performed in human subjects. EE may provide a more valid and predictive animal model for pre-clinical drug screening compared to highly impoverished standard housing conditions. Additionally, it would significantly increase animal welfare (Wolfer, et al., 2004). For future studies, it would be interesting to investigate whether drugs that improve memory performance in humans are also effective in $\mathrm{EE}$ rats. This would provide further insight into the validity of SOL/SOC versus EE rats as an animal model for testing cognition enhancing drugs. 


\section{REFERENCES}

Akar, F., Mutlu, O., Celikyurt, I. K., Ulak, G., Erden, F., Bektas, E., et al. (2014). Effects of Rolipram and Zaprinast on Learning and Memory in the Morris Water Maze and Radial Arm Maze Tests in Naive Mice. Drug Res (Stuttg)(EFirst).

Akar, F., Mutlu, O., Komsuoglu Celikyurt, I., Ulak, G., Erden, F., Bektas, E., et al. (2014). Zaprinast and rolipram enhances spatial and emotional memory in the elevated plus maze and passive avoidance tests and diminishes exploratory activity in naive mice. Med Sci Monit Basic Res, 20, 105-111.

Akkerman, S., Blokland, A., \& Prickaerts, J. (2014). Mind the gap: Delayed manifestation of long-term object memory improvement by phosphodiesterase inhibitors. Neurobiol Learn Mem, 109C, 139-143.

Akkerman, S., Blokland, A., Reneerkens, O., van Goethem, N. P., Bollen, E., Gijselaers, H. J., et al. (2012). Object recognition testing: Methodological considerations on exploration and discrimination measures. Behav Brain Res, 232(2), 335347.

Akkerman, S., Blokland, A., Reneerkens, O., van Goethem, N. P., Bollen, E., Gijselaers, H. J. M., et al. (2012). Object recognition testing: Methodological considerations on exploration and discrimination measures. Behav Brain Res, 232(2), 335-347.

Akkerman, S., Prickaerts, J., Steinbusch, H. W. M., \& Blokland, A. (2012). Object recognition testing: Statistical considerations. Behav Brain Res, 232(2), 317322.

Andin, J., Hallbeck, M., Mohammed, A. H., \& Marcusson, J. (2007). Influence of environmental enrichment on steady-state mRNA levels for EAAC1, AMPA1 and NMDA2A receptor subunits in rat hippocampus. Brain Research, 1174, 18-27.

Arndt, D. L., Arnold, J. C., \& Cain, M. E. (2014). The effects of mGluR2/3 activation on acute and repeated amphetamine-induced locomotor activity in differentially reared male rats. Exp Clin Psychopharmacol, 22(3), 257-265.

Bannerman, D. M., Rawlins, J. N., McHugh, S. B., Deacon, R. M., Yee, B. K., Bast, T., et al. (2004). Regional dissociations within the hippocampus--memory and anxiety. Neurosci Biobehav Rev, 28(3), 273-283.

Bechara, R. G., \& Kelly, A. M. (2013). Exercise improves object recognition memory and induces BDNF expression and cell proliferation in cognitively enriched rats. Behav Brain Res, 245, 96-100.

Bekinschtein, P., Oomen, C. A., Saksida, L. M., \& Bussey, T. J. (2011). Effects of environmental enrichment and voluntary exercise on neurogenesis, learning and memory, and pattern separation: BDNF as a critical variable? Semin Cell Dev Biol, 22(5), 536-542. 
Bindu, B., Alladi, P. A., Mansooralikhan, B. M., Srikumar, B. N., Raju, T. R., \& Kutty, B. M. (2007). Short-term exposure to an enriched environment enhances dendritic branching but not brain-derived neurotrophic factor expression in the hippocampus of rats with ventral subicular lesions. Neuroscience, 144(2), 412-423.

Boccia, M. M., Blake, M. G., Krawczyk, M. C., \& Baratti, C. M. (2011). Sildenafil, a selective phosphodiesterase type 5 inhibitor, enhances memory reconsolidation of an inhibitory avoidance task in mice. Behav Brain Res, 220(2), 319-324.

Bowling, S. L., Rowlett, J. K., \& Bardo, M. T. (1993). The effect of environmental enrichment on amphetamine-stimulated locomotor activity, dopamine synthesis and dopamine release. Neuropharmacology, 32, 885-893.

Bruel-Jungerman, E., Laroche, S., \& Rampon, C. (2005). New neurons in the dentate gyrus are involved in the expression of enhanced long-term memory following environmental enrichment. European Journal of Neuroscience, 21, 513-521.

Cao, W., Duan, J., Wang, X., Zhong, X., Hu, Z., Huang, F., et al. (2014). Early enriched environment induces an increased conversion of proBDNF to BDNF in the adult rat's hippocampus. Behavioural Brain Research(0).

Chourbaji, S., Hortnagl, H., Molteni, R., Riva, M. A., Gass, P., \& Hellweg, R. (2012). The impact of environmental enrichment on sex-specific neurochemical circuitries - effects on brain-derived neurotrophic factor and the serotonergic system. Neuroscience, 220, 267-276.

Del Arco, A., Segovia, G., Canales, J. J., Garrido, P., de Blas, M., Garcia-Verdugo, J. M., et al. (2007). Environmental enrichment reduces the function of D1 dopamine receptors in the prefrontal cortex of the rat. Journal of Neural Transmission, 114, 43-48.

Devan, B. D., Sierra-Mercado, D., Jr., Jimenez, M., Bowker, J. L., Duffy, K. B., Spangler, E. L., et al. (2004). Phosphodiesterase inhibition by sildenafil citrate attenuates the learning impairment induced by blockade of cholinergic muscarinic receptors in rats. Pharmacol Biochem Behav, 79(4), 691-699.

Diniz, D. G., Foro, C. A., Rego, C. M., Gloria, D. A., de Oliveira, F. R., Paes, J. M., et al. (2010). Environmental impoverishment and aging alter object recognition, spatial learning, and dentate gyrus astrocytes. Eur J Neurosci, 32(3), 509-519.

Ennaceur, A. (2010). One-trial object recognition in rats and mice: Methodological and theoretical issues. Behavioural Brain Research, 215(2), 244-254.

Escorihuela, R. M., Fernandez-Teruel, A., Tobena, A., Vivas, N. M., Marmol, F., Badia, A., et al. (1995). Early environmental stimulation produces long-lasting changes on beta-adrenoceptor transduction system. Neurobiol Learn Mem, 64(1), 49-57. 
Faherty, C. J., Kerley, D., \& Smeyne, R. J. (2003). A Golgi-Cox morphological analysis of neuronal changes induced by environmental enrichment. Brain Res Dev Brain Res, 141(1-2), 55-61.

Galani, R., Berthel, M. C., Lazarus, C., Majchrzak, M., Barbelivien, A., Kelche, C., et al. (2007). The behavioral effects of enriched housing are not altered by serotonin depletion but enrichment alters hippocampal neurochemistry. Neurobiol Learn Mem, 88(1), 1-10.

Gaskin, S., Tardif, M., Cole, E., Piterkin, P., Kayello, L., \& Mumby, D. G. (2010). Object familiarization and novel-object preference in rats. Behav Processes, 83(1), 61-71.

Grass, H., Klotz, T., Fathian-Sabet, B., Berghaus, G., Engelmann, U., \& Kaferstein, H. (2001). Sildenafil (Viagra): is there an influence on psychological performance? Int Urol Nephrol, 32(3), 409-412.

Grimes, C. A., \& Jope, R. S. (2001). The multifaceted roles of glycogen synthase kinase 3beta in cellular signaling. Prog Neurobiol, 65(4), 391-426.

Gurwitz, D. (2001). Are drug targets missed owing to lack of physical activity? Drug Discov Today, 6(7), 342-343.

Harati, H., Barbelivien, A., Herbeaux, K., Muller, M. A., Engeln, M., Kelche, C., et al. (2013). Lifelong environmental enrichment in rats: impact on emotional behavior, spatial memory vividness, and cholinergic neurons over the lifespan. Age (Dordr), 35(4), 1027-1043.

Harati, H., Majchrzak, M., Cosquer, B., Galani, R., Kelche, C., Cassel, J. C., et al. (2011). Attention and memory in aged rats: Impact of lifelong environmental enrichment. Neurobiol Aging, 32(4), 718-736.

Hebb, D. O. (1947). The effects of early experience on problem solving at maturity. American Psychologist, 2, 306-307.

Hellemans, K. G., Nobrega, J. N., \& Olmstead, M. C. (2005). Early environmental experience alters baseline and ethanol-induced cognitive impulsivity: relationship to forebrain 5-HT1A receptor binding. Behavioural Brain Research, 159, 207-220.

Hicks, R. R., Zhang, L., Atkinson, A., Stevenon, M., Veneracion, M., \& Seroogy, K. B. (2002). Environmental enrichment attenuates cognitive deficits, but does not alter neurotrophin gene expression in the hippocampus following lateral fluid percussion brain injury. Neuroscience, 112(3), 631-637.

Hosseini-Sharifabad, A., Ghahremani, M. H., Sabzevari, O., Naghdi, N., Abdollahi, M., Beyer, C., et al. (2012). Effects of protein kinase A and $G$ inhibitors on hippocampal cholinergic markers expressions in rolipram- and sildenafilinduced spatial memory improvement. Pharmacol Biochem Behav, 101(3), 311-319.

Hutchinson, E., Avery, A., \& Vandewoude, S. (2005). Environmental enrichment for laboratory rodents. ILAR J, 46(2), 148-161. 
Ickes, B. R., Pham, T. M., Sanders, L. A., Albeck, D. S., Mohammed, A. H., \& Granholm, A. C. (2000). Long-term environmental enrichment leads to regional increases in neurotrophin levels in rat brain. Exp Neurol, 164(1), 4552.

Jin, F., Gong, Q. H., Xu, Y. S., Wang, L. N., Jin, H., Li, F., et al. (2014). Icariin, a phosphodiesterase-5 inhibitor, improves learning and memory in APP/PS1 transgenic mice by stimulation of NO/CGMP signalling. Int $J$ Neuropsychopharmacol, 17(6), 871-881.

Kazlauckas, V., Pagnussat, N., Mioranzza, S., Kalinine, E., Nunes, F., Pettenuzzo, L., et al. (2011). Enriched environment effects on behavior, memory and BDNF in low and high exploratory mice. Physiol Behav, 102(5), 475-480.

Kempermann, G., Kuhn, H. G., \& Gage, F. H. (1997). More hippocampal neurons in adult mice living in an enriched environment. Nature, 386(6624), 493-495.

Kobilo, T., Liu, Q. R., Gandhi, K., Mughal, M., Shaham, Y., \& van Praag, H. (2011). Running is the neurogenic and neurotrophic stimulus in environmental enrichment. Learn Mem, 18(9), 605-609.

Kobilo, T., Yuan, C., \& van Praag, H. (2011). Endurance factors improve hippocampal neurogenesis and spatial memory in mice. Learn Mem, 18(2), 103-107.

Laviola, G., Hannan, A. J., Macri, S., Solinas, M., \& Jaber, M. (2008). Effects of enriched environment on animal models of neurodegenerative diseases and psychiatric disorders. Neurobiology of Disease, 31, 159-168.

Leger, M., Quiedeville, A., Paizanis, E., Natkunarajah, S., Freret, T., Boulouard, M., et al. (2012). Environmental enrichment enhances episodic-like memory in association with a modified neuronal activation profile in adult mice. PLOS One, 7(10), e48043.

Lynch, G., \& Gall, C. M. (2013). Mechanism based approaches for rescuing and enhancing cognition. Front Neurosci, 7, 143.

Moser, M. B., Trommald, M., \& Andersen, P. (1994). An increase in dendritic spine density on hippocampal CA1 pyramidal cells following spatial learning in adult rats suggests the formation of new synapses. Proc Natl Acad Sci U S A, 91(26), 12673-12675.

Mumby, D. G. (2001). Perspectives on object-recognition memory following hippocampal damage: lessons from studies in rats. Behavioural Brain Research, 127(1-2), 159-181.

Myhrer, T. (2003). Neurotransmitter systems involved in learning and memory in the rat: a meta-analysis based on studies of four behavioral tasks. Brain Research Reviews, 41(23), 268-287.

Nithianantharajah, J., Levis, H., \& Murphy, M. (2004). Environmental enrichment results in cortical and subcortical changes in levels of synaptophysin and PSD95 proteins. Neurobiol Learn Mem, 81(3), 200-210. 
Okuda, T., Yu, L. M., Cingolani, L. A., Kemler, R., \& Goda, Y. (2007). beta-Catenin regulates excitatory postsynaptic strength at hippocampal synapses. Proc Natl Acad Sci U S A, 104(33), 13479-13484.

Pamplona, F. A., Pandolfo, P., Savoldi, R., Prediger, R. D., \& Takahashi, R. N. (2009). Environmental enrichment improves cognitive deficits in Spontaneously Hypertensive Rats (SHR): relevance for Attention Deficit/Hyperactivity Disorder (ADHD). Progress in Neuropsychopharmacology \& Biological Psychiatry, 33, 1153-1160.

Pang, P. T., Teng, H. K., Zaitsev, E., Woo, N. T., Sakata, K., Zhen, S., et al. (2004). Cleavage of proBDNF by tPA/plasmin is essential for long-term hippocampal plasticity. Science, 306(5695), 487-491.

Paxinos, G., \& Watson, C. (1998). The Rat Brain in Stereotaxic Coordinates. Waltham, MA: Academic Press.

Prickaerts, J., de Vente, J., Honig, W., Steinbusch, H. W., \& Blokland, A. (2002). CGMP, but not CAMP, in rat hippocampus is involved in early stages of object memory consolidation. Eur J Pharmacol, 436(1-2), 83-87.

Prickaerts, J., Sik, A., van Staveren, W. C. G., Koopmans, G., Steinbusch, H. W. M., van der Staay, F. J., et al. (2004). Phosphodiesterase type 5 inhibition improves early memory consolidation of object information. Neurochemistry International, 45(6), 915-928.

Prickaerts, J., van Staveren, W. C. G., Sik, A., Markerink-van Ittersum, M., Niewöhner, U., van der Staay, F. J., et al. (2002). Effects of two selective phosphodiesterase type 5 inhibitors, sildenafil and vardenafil, on object recognition memory and hippocampal cyclic GMP levels in the rat. Neuroscience, 113(2), 351-361.

Ramirez-Rodriguez, G., Ocana-Fernandez, M. A., Vega-Rivera, N. M., Torres-Perez, O. M., Gomez-Sanchez, A., Estrada-Camarena, E., et al. (2014). Environmental enrichment induces neuroplastic changes in middle age female BalbC mice and increases the hippocampal levels of BDNF, p-Akt and p-MAPK1/2. Neuroscience, 260, 158-170.

Reneerkens, O. A., Rutten, K., Akkerman, S., Blokland, A., Shaffer, C. L., Menniti, F. S., et al. (2012). Phosphodiesterase type 5 (PDE5) inhibition improves object recognition memory: indications for central and peripheral mechanisms. Neurobiol Learn Mem, 97(4), 370-379.

Reneerkens, O. A., Rutten, K., Steinbusch, H. W., Blokland, A., \& Prickaerts, J. (2009). Selective phosphodiesterase inhibitors: a promising target for cognition enhancement. Psychopharmacology (Berl), 202(1-3), 419-443.

Reneerkens, O. A., Sambeth, A., Ramaekers, J. G., Steinbusch, H. W., Blokland, A., \& Prickaerts, J. (2013). The effects of the phosphodiesterase type 5 inhibitor vardenafil on cognitive performance in healthy adults: a behavioralelectroencephalography study. J Psychopharmacol, 27(7), 600-608. 
Rosenzweig, M. R., \& Bennett, E. L. (1972). Cerebral changes in rats exposed individually to an enriched environment. Journal of Comparative Physiological Psychology, 80, 304-313.

Rutten, K., Prickaerts, J., Hendrix, M., van der Staay, F. J., Sik, A., \& Blokland, A. (2007). Time-dependent involvement of cAMP and CGMP in consolidation of object memory: studies using selective phosphodiesterase type 2, 4 and 5 inhibitors. Eur J Pharmacol, 558(1-3), 107-112.

Rutten, K., Van Donkelaar, E. L., Ferrington, L., Blokland, A., Bollen, E., Steinbusch, H. W., et al. (2009). Phosphodiesterase inhibitors enhance object memory independent of cerebral blood flow and glucose utilization in rats. Neuropsychopharmacology, 34(8), 1914-1925.

Sarter, M. (2006). Preclinical research into cognition enhancers. Trends in Pharmacological Sciences, 27, 602-808.

Schmitz, C., \& Hof, P. R. (2005). Design-based stereology in neuroscience. Neuroscience, 130(4), 813-831.

Schmitz, C., Rutten, B. P., Pielen, A., Schafer, S., Wirths, O., Tremp, G., et al. (2004). Hippocampal neuron loss exceeds amyloid plaque load in a transgenic mouse model of Alzheimer's disease. Am J Pathol, 164(4), 1495-1502.

Schultheiss, D., Muller, S. V., Nager, W., Stief, C. G., Schlote, N., Jonas, U., et al. (2001). Central effects of sildenafil (Viagra) on auditory selective attention and verbal recognition memory in humans: a study with event-related brain potentials. World J Urol, 19(1), 46-50.

Sierksma, A. S., Vanmierlo, T., De Vry, J., Raijmakers, M. E., Steinbusch, H. W., van den Hove, D. L., et al. (2012). Effects of prenatal stress exposure on soluble Abeta and brain-derived neurotrophic factor signaling in male and female APPswe/PS1dE9 mice. Neurochem Int, 61(5), 697-701.

Simpson, J., Bree, D., \& Kelly, J. P. (2012). Effect of early life housing manipulation on baseline and drug-induced behavioural responses on neurochemistry in the male rat. Progress in Neuro-Psychopharmacology and Biological Psychiatry, $37(2), 252-263$.

Simpson, J., \& Kelly, J. P. (2011). The impact of environmental enrichment in laboratory rats- Behavioural and neurochemical aspects. Behavioural Brain Research, 222(1), 246-264.

Simpson, J., \& Kelly, J. P. (2012). The effects of isolated and enriched housing conditions on baseline and drug-induced behavioural responses in the male rat. Behavioural Brain Research, 234(2), 175-183.

Thouvarecq, R., Caston, J., \& Protais, P. (2007). Cholinergic system, rearing environment and trajectory learning during aging in mice. Physiology and Behavior, 90, 155-164. 
Turner, C. A., \& Lewis, M. H. (2003). Environmental enrichment: effects on stereotyped behavior and neurotrophin levels. Physiol Behav, 80(2-3), 259266.

Uthayathas, S., Parameshwaran, K., Karuppagounder, S. S., Ahuja, M., Dhanasekaran, M., \& Suppiramaniam, V. (2013). Selective inhibition of phosphodiesterase 5 enhances glutamatergic synaptic plasticity and memory in mice. Synapse, $67(11), 741-747$.

van Donkelaar, E. L., Rutten, K., Blokland, A., Akkerman, S., Steinbusch, H. W. M., \& Prickaerts, J. (2008). Phosphodiesterase 2 and 5 inhibition attenuates the object memory deficit induced by acute tryptophan depletion. European Journal of Pharmacology, 600(1-3), 98-104.

van Praag, H., Barlow, C., \& Gage, F. H. (2001). Are drug targets missed owing to lack of physical activity? Drug Discovery Today, 6, 615-617.

Varela-Nallar, L., \& Inestrosa, N. C. (2013). Wnt signaling in the regulation of adult hippocampal neurogenesis. Front Cell Neurosci, 7, 100.

Vedovelli, K., Silveira, E., Velho, E., Stertz, L., Kapczinski, F., Schroder, N., et al. (2011). Effects of increased opportunity for physical exercise and learning experiences on recognition memory and brain-derived neurotrophic factor levels in brain and serum of rats. Neuroscience, 199, 284-291.

Viola, G. G., Botton, P. H., Moreira, J. D., Ardais, A. P., Oses, J. P., \& Souza, D. O. (2010). Influence of environmental enrichment on an object recognition task in CF1 mice. Physiol Behav, 99(1), 17-21.

Viola, G. G., \& Loss, C. M. (2014). Letter to Editor about: "Physical exercise increases GFAP expression and induces morphological changes in hippocampal astrocytes". Brain Struct Funct, 219(4), 1509-1510.

Vivar, C., Potter, M. C., \& van Praag, H. (2013). All about running: synaptic plasticity, growth factors and adult hippocampal neurogenesis. Curr Top Behav Neurosci, 15, 189-210.

Wolfer, D. P., Litvin, O., Morf, S., Nitsch, R. M., Lipp, H. P., \& Wurbel, H. (2004). Laboratory animal welfare: cage enrichment and mouse behaviour. Nature, 432(7019), 821-822.

Yildirim, E., Erol, K., \& Ulupinar, E. (2012). Effects of sertraline on behavioral alterations caused by environmental enrichment and social isolation. Pharmacology Biochemistry and Behavior, 101, 278-287.

Young, D., Lawlor, P. A., Leone, P., Dragunow, M., \& During, M. J. (1999). Environmental enrichment inhibits spontaneous apoptosis, prevents seizures and is neuroprotective. Nat Med, 5(4), 448-453.

Zeeb, F. D., Wong, A. C., \& Winstanley, C. A. (2013). Differential effects of environmental enrichment, social-housing, and isolation-rearing on a rat gambling task: Dissociations between impulsive action and risky decisionmaking. Psychopharmacology, 225, 381-395. 



\section{ChAPTER 8}




\section{General Discussion}


The aim of this thesis was to extend our knowledge about second messenger signaling during consolidation processes, in particular their temporal characteristics in vivo. For this research we used the ORT, which is a widely used method to assess spontaneous memory performance in animals. The great advantage of this one-trial learning tasks is that acquisition and early- and late-consolidation processes can be examined independently. We started with an extensive investigation of the ORT to assess the sensitivity, relationship between exploration and discrimination measures and type of memory that is measured (Chapter 2). Furthermore, we compared the different discrimination indices, to gain further insight in how to properly analyze and interpret results of the ORT and compare findings between studies (Chapter 3).

Previous studies have used PDE4-Is and PDE5-Is to investigate the involvement of their downstream targets, CAMP and CGMP, in different stages of memory formation and used very specific injection time points in relation the learning trial (Prickaerts, de Vente, Honig, Steinbusch, \& Blokland, 2002; Prickaerts, van Staveren et al., 2002; Rutten et al., 2007). These specific administration times do not give much information about the precise window of activity of their intracellular targets. In this thesis we wanted to further elaborate the signaling windows of CAMP and CGMP in vivo. Therefore, we investigated the pharmacokinetic profiles of the PDE4-I rolipram and PDE5-I vardenafil and tested these drugs at various time points in the ORT to discover the earliest and latest effective time of injection, hence revealing the total window of action of central CAMP and CGMP signaling during memory formation (Chapter 4 and $5)$.

It is known that memories are dynamic over time and that structural changes occur in the brain for hours and even days after new information has been acquired (Bailey, Kandel, \& Harris, 2015). However, not much is known about the expression of memories during these initial stages of memory. In Chapter 6 we investigated the expression of memory over the first $24 \mathrm{~h}$ after the learning trial in the ORT. In placebo-treated animals we observed normal forgetting and, as expected (Rutten et al., 2007), memory performance at $24 \mathrm{~h}$ was improved after treatment with PDEinhibitors. However, this memory improvement was not expressed at intervals shorter 
than $10 \mathrm{~h}$, indicating that memory undergoes a transition from early- to long-term expression.

There are many pre-clinical studies that have shown pro-cognitive effects of drugs in various animal models. However, the clinical potential of most of these drugs appears to be very limited. One of the major causes of attrition in drug development is a lack of efficacy in the clinical phase and especially with regards to the CNS, animal studies have proven to be notoriously unpredictive (Kola \& Landis, 2004). This raises concerns about the potential of PDE-Is as cognition enhancers as all current pro-cognitive findings are derived from animal studies. Therefore, in Chapter 7, we explored whether environmental factors can affect the efficacy of PDE5-I vardenafil and explain a portion of the discrepancy between human- and animal studies.

\section{THE CHARACTERISTICS OF THE OBJECt RECOGNITION TASK}

When properly performed, the ORT is believed a sensitive, reliable memory assessment tool that is able to detect subtle behavioral and cognitive effects. One of the main assumptions of the ORT is that it exploits the animal's instinctive attraction towards novelty, or neotic preference (Ennaceur \& Delacour, 1988; Hughes, 2007). For this reason, it does not require any form of positive or negative reinforcement. This hypothesis that was confirmed by our observation that naïve animals immediately discriminated between objects when first introduced to the ORT.

We found that naive animals required at least 9-10 $\mathrm{s}$ of object interaction in T1 and $12 \mathrm{~s}$ in $\mathrm{T} 2$ for reliable object discrimination, fully habituated animals only needed between 6-7 s and 9-10 s of object exploration in $\mathrm{T} 1$ and $\mathrm{T} 2$, respectively. In accordance with Dix and Aggleton (1999) we found that most (discriminative) object exploration takes place in the initial 2 min of a trial. This implies that only a short learning trial is required to induce the formation of memory and makes the ORT a very useful instrument to temporally dissect different stages of memory formation that allows for the separation of different memory processes, like acquisition, consolidation and retrieval (Prickaerts, Sik, van der Staay, de Vente, \& Blokland, 2005; Rutten et al., 2007). In all our experiments we used short fixed trials of 3 min, ensuring a minimal 
dropout rate due to insufficient exploration. Fixed trial durations are an appropriate way to compare memory performance because we found that $\mathrm{e} 1$ is not correlated to object discrimination, therefore it was unnecessary to correct for natural differences in exploratory activity between individual animals. The same was found for the $\mathrm{d} 2$ discrimination parameter which additionally corrects for possible exploration differences during T2. Another argument for the use of a relative discrimination measure, like the $d 2$, is that it enables the comparison between studies from different laboratories and even compare them to studies using a animals from a different sex or strain, and even to other variants of the ORT (van Goethem et al., 2012).

\section{PHARMACOLOGY AND THE ORT}

The formation of memories requires a sequence of complementary processes to register, maintain and retrieve information. Therefore, it is common to segregate memory processes into an acquisition, consolidation and retrieval phase. When testing cognition enhancing properties of drugs, it is particularly relevant to dissociate its effects on these memory stages, particularly given the accumulating evidence that these different memory processes rely on distinct underlying neurobiological mechanisms (Izquierdo et al., 2006; Prickaerts et al., 2005; Winters, Saksida, \& Bussey, 2008).

The ORT is especially suited to separate short-term memory and long-term memory (STM and LTM, respectively). For example, when using a short retention interval, i.e. $10 \mathrm{~min}$ to $1 \mathrm{~h}$, one is testing STM whereas longer retention intervals, like $24 \mathrm{~h}$ are useful to asses LTM performance. Pharmacological treatments close before T1 will, depending on the pharmacokinetics of the drug, affect acquisition and earlyconsolidation, whereas treatments shortly after T1 will only affect early consolidation processes. When drugs are injected at longer intervals after T1, late-consolidation will be affected as early-consolidation processes finish within the first $3 \mathrm{~h}$ after a stimulus (Abel \& Lattal, 2001). Finally, retrieval and possibly re-consolidation processes can be targeted when injecting drugs shortly before $\mathrm{T} 2$ whereas treating animals after $\mathrm{T} 2$ will only affect re-consolidation processes. In this way the formation of memory can be elegantly dissected, targeting or excluding particular memory processes using the 
duration of the retention interval and/or the timing of the intervention. However, when making inferences about the memory processes that are affected by drug treatments, it is of crucial importance to know the pharmacokinetics of the test compound, before making any conclusions about the memory stages affected.

\section{PHARMACOKINETICS OF DONEPEZIL, VARDENAFIL AND ROLIPRAM}

\section{IN THE RAT}

We decided to investigate the effective windows of 3 different types of nootropics that are known to affect different phases of memory formation: the cholinesterase inhibitor (AChE-I) donepezil, the PDE5-I vardenafil, and the PDE4-I rolipram. We investigated the pharmacokinetic profile of these drugs in the same rat species and with the exact same administration procedures as were used in the behavioral experiments. This is important as pharmacokinetics can be greatly affected by these factors. One of the most important questions was whether or not drugs are brain penetrant in rats.

As the unbound brain-to-unbound plasma drug concentration ratio is most important for defining pharmacological exposure-response relationships, we based our conclusions on the unbound drug concentrations (Shaffer \& John, 2010). Considering the proportion of cerebral blood within the total brain volume, a drug is considered brain penetrant if its brain/plasma drug concentration ratio is $>0.04$ (Hitchcock \& Pennington, 2006). Based on these generally accepted assumptions we concluded that all three drugs were abundantly present in the brain as our measurements showed that the minimum unbound brain/plasma ratios of donepezil, vardenafil and rolipram were $6.40,0.45$ and 2.50 , respectively. After confirming that all compounds were able to successfully cross the blood brain barrier, our next objective was to determine how fast each of these drugs would become centrally available.

We detected donepezil in the brain within 4 min after injection ( $1 \mathrm{mg} / \mathrm{kg}, \mathrm{PO}$ ). Free brain concentrations above IC50 were reached between 10-60 min after administration and the highest free brain concentration was measured 20 min after the oral injection. These measurements are in line with observations from other 
researchers who reported that maximum plasma and brain concentrations are reached simultaneously at approximately 30 min after PO donepezil administration (1 mg/kg) with a half-life of about 1 h (Kenji Matsui, Mannen Mishima, Yasushi Nagai, Teruaki Yuzuriha, \& Tsutomu Yoshimura, 1999).

Few studies have been published about the pharmacokinetic properties of vardenafil in rats, especially with regards to oral administration. Unfortunately, we did not find any studies to use as a proper reference. In our own study vardenafil was detected in the brain 2 min after injection ( $1 \mathrm{mg} / \mathrm{kg}, \mathrm{PO}$ ) and unbound brain levels of vardenafil were above IC50 between 10-60 min after administration. The highest central concentration of vardenafil was measured 20 min after administration.

Rolipram has been reported to reach maximum plasma levels $0.25 \mathrm{~h}$ after oral administration (Krause \& kuhne, 1988). In our study rolipram was detected in the brain 2 min after injection $(0.03 \mathrm{mg} / \mathrm{kg}, \mathrm{IP})$ and reached unbound brain concentrations above IC50 between 4-60 min after injection. The highest central concentration of rolipram was detected at 6 min after injection.

\section{PHARMACOLOGY AND BEHAVIOR}

Scopolamine is a muscarinic receptor antagonist which is used to mimic cognitive deficits in Alzheimer's disease (Klinkenberg \& Blokland, 2010). In our ORT experiments with a $1 \mathrm{~h}$ and 10 min retention interval we used scopolamine, injected 30 min before T1, to suppress memory acquisition. The drug was able to fully abolish object discrimination without large effects on locomotor activity. It has been shown that post-learning blockage of muscarinic receptors does not impair memory consolidation processes (Rush, 1988; Spangler, Chachich, \& Ingram, 1988; Young, Bohenek, \& Fanselow, 1995). In addition, it has been shown that scopolamine infusions in the perirhinal cortex, immediately and up to $20 \mathrm{~h}$, post sample-trial improve object discrimination in a spontaneous object recognition task (Winters, Saksida, \& Bussey, 2006). Therefore, we can assume that the memory impairment induced by scopolamine is largely acquisition related (Blake, Boccia, Krawczyk, \& Baratti, 2011). 
We observed that donepezil was able to restore memory function when injected up to 2 min after T1. This was surprising, as studies have shown that AChE-Is enhance acquisition processes but not (early) consolidation processes. In combination with our pharmacokinetic measurements this finding implies that the performance enhancing effects of ACh, which in high concentrations is assumed to facilitate the acquisition of new information (Rasch, Born, \& Gais, 2006), continue for 6 or 12 min after T1 (Figure 1). The exact of this time window depends on whether one bases conclusions merely on central availability or concentration levels exceeding the IC50. Because we found the exact same window of treatment for donepezil in the $24 \mathrm{~h}$ retention interval, we can exclude an effect on retrieval processes as donepezil has a half-life of 1 h (Matsui, Mishima, Nagai, Yuzuriha, \& Yoshimura, 1999). The surprisingly similar effective windows of vardenafil and rolipram treatment in the scopolamine challenge also indicate an involvement of their downstream targets, CGMP and CAMP, in acquisition processes.

PDE5-Is, like vardenafil, are known to affect early-consolidation and acquisition processes by enhancing intracellular cyclic guanosine monophosphate (cGMP)/Protein kinase G (PKG) signaling (Bollen et al., 2014; Devan et al., 2007; Devan et al., 2004; Domek-Lopacinska \& Strosznajder, 2008; Prickaerts et al., 2005; van Donkelaar et al., 2008). We found that vardenafil effectively enhanced ORT performance in the $24 \mathrm{~h}$ interval when injected within $45 \mathrm{~min}$ after T1. From our pharmacokinetic measurements we know that vardenafil requires 2-10 min to become centrally available. Hence, we can conclude that that the cGMP pathway becomes involved in LTM during T1 and remains activated until roughly 47-55 min after T1 (Figure1), which fits nicely with the time window of cGMP elevation during memory formation (Izquierdo et al., 2006).

Finally, the PDE4-Is, like rolipram, improve cyclic adenosine monophosphate (CAMP)/Protein kinase A (PKA) signaling, which are involved in acquisition and lateconsolidation processes when administered before learning or at $3 \mathrm{~h}$ after (Bollen et al., 2014; Rutten, Prickaerts, \& Blokland, 2006; Rutten et al., 2007). As we found that rolipram reaches the brain very rapidly, within 2 min and IC50 within 4 min after 
injection, we can deduce that the PKA signaling pathway remains involved in acquisition until 6 min after T1. Later during LTM formation the PKA pathway again becomes crucially involved. We discovered that his window extends from roughly $3 \mathrm{~h}$ until $5.5 \mathrm{~h}$ after T1 (Figure 1). Again, these findings align nicely with observed elevation of cAMP elevation during memory formation (Izquierdo et al., 2006).

Our findings on the windows of effect of donepezil, vardenafil and rolipram suggest a temporal overlap, of at least 4-6 min, between the acquisition and early consolidation phases of memory. This window might represent a transition between acquisition processes and the consolidation stage of memory. The animals were unable to acquire object information after T1, as they were returned to their home cage immediately after testing. Also, as described above, it is unlikely that consolidation is enhanced by donepezil treatment. Therefore, it is possible that there are encoding processes continuing for a brief period of time after stimulus exposure. Encoding refers to the formation of relations between events in episodic memory, based on various kinds of information, such as temporal and categorical information (Lepage, Habib, Cormier, Houle, \& Mclntosh, 2000). It also entails novelty/familiarity evaluation (Habib, Mclntosh, Wheeler, \& Tulving, 2003), and involves trans-perceptual processes operate beyond perceptual processes (Lepage, Mclntosh, \& Tulving, 2001).

\section{EXPRESSION OF OBJECT MEMORY OVER TIME}

It is known that cGMP-specific PDE5 inhibitors (PDE5-I) are effective when injected shortly before learning or immediately after learning. CAMP-specific PDE4 inhibitors (PDE4-I) also improve memory performance but only when given $3 \mathrm{~h}$ after learning (Bernabeu et al., 1997; Prickaerts, de Vente, Honig, Steinbusch, \& Blokland, 2002; Devan et al., 2004; Rutten et al., 2006; Rutten et al., 2007; Levallet, Hotte, Boulouard, \& Dauphin, 2009; Bruno et al., 2011; Reneerkens et al., 2012). However, not much was known about how LTM enhanced by PDE-Is is expressed over time. Therefore we investigated the expression of memory, enhanced by vardenafil or rolipram, in the ORT over the first $24 \mathrm{~h}$ after T1. Based on our discovery of a 2 min post- window, during which acquisition related processes are enhanced, we ensured that only (early) 
consolidation processes were affected by injecting vardenafil 4 min after T1 and rolipram $3 \mathrm{~h}$ after $\mathrm{T}$.

Remarkably, within the first $8 \mathrm{~h}$ after the learning trial PDE-I treated animals showed exactly the same decline in discrimination as vehicle treated animals. Only after $10 \mathrm{~h}$ the memory enhancing effects of the drugs emerged and these effects remained present until $24 \mathrm{~h}$ after the learning trial. This shows that the positive effect of both PDE4- and PDE5-Is is not expressed until well after the active window of their downstream targets. In addition, these findings reveal that the expression of a memory effect in the ORT is very much time-dependent and show that two distinct forms of memory seem to be involved in the $24 \mathrm{~h}$ retention interval. The CGMP and CAMP signaling pathways are both involved in LTM formation and appear to converge on the same downstream target in the memory consolidation process, as both displayed identical memory expression profiles. This observation fits neatly with a study by Bollen et. al. (2014) showing that LTM improvement via CGMP/PKG stimulation is dependent on CAMP/PKA signaling. Furthermore, in another study we found that both PDE4-Is and PDE5-Is work synergistically when administered in combination, even at doses that are sub-efficacious when the drugs used individually (Bollen et al., 2015). From the current experiment, we the underlying expression of genes, proteins synthesis and strutural network changes needed for long-term storage of information requires at least $10 \mathrm{~h}$. The expression of memory within the first $24 \mathrm{~h}$ in the ORT, combined with the activity windows of ACh, CGMP and CAMP is shown in Figure 1.

The observed memory 'gap' is not likely to be a transition phase in between STM and LTM, as it is manifested well after the assumed short term memory phase. It is possible that two distinct memory representations are involved. One rapid transient form that degrades within $8 \mathrm{~h}$ after learning and a stable form integrating episodic information into the existing semantic structure, which gradually builds at a neurobiological level and becomes manifest only after 10 h (Kamin, 1957; Sutton \& Carew, 2002; Rudy \& Wright-Hardesty, 2005; Tang et al., 2007). 
A major implication of the present findings is that positive effects of drugs on longterm memory may sometimes go unnoticed due to the time-dependency of their effects.

Non-structural changes

T1

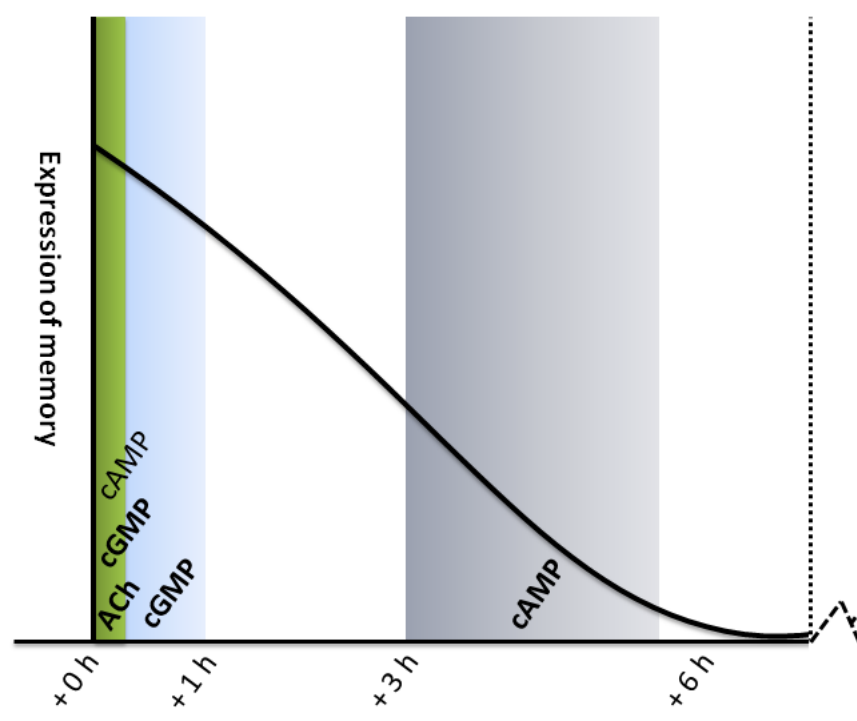

Time after T1
Structural changes

T2

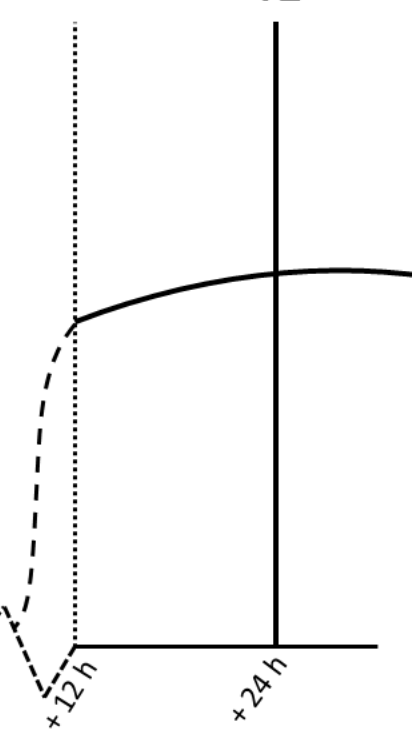

Figure 1 Expression of memory and active windows of messenger molecules in the ORT

During the first $8 \mathrm{~h}$ after the learning trial $(T 1)$ object discrimination declines, regardless of drug treatment (vehicle, vardenafil or rolipram) or whether or not the treatment led to object discrimination at a longer time interval. After treatment with vardenafil or rolipram, object discrimination re-appeared at $10 \mathrm{~h}$ after T1 and remained increasing up to $24 \mathrm{~h}$ after T1. Based on injection timing and pharmacokinetic properties of AChE-I donepezil, PDE5-I vardenafil and PDE4-I rolipram we were able to determine the activity windows of ACh, CGMP and CAMP, respectively. The estimated window of action of ACh was 0-6/12 min after T1. Vardenafil revealed 2 windows of action for CGMP, an initial window of 0-2/10 min directly after $T 1$, overlapping with that of $\mathrm{ACh}$ and a second one from 0-47/55 min after T1. CAMP also appeared to have an initial short window of action of 0-4/6 min after T1, overlapping with that of ACh and CGMP, as well as long late activity window for 3-5.5h after T1. The short estimates of the windows is based on the shortest time it takes the drug to become centrally available, the long estimate is based on the time it takes the central unbound drug concentration to reach IC 50 .

\section{THE EFFECTS OF ENVIRONMENTAL ENRICHMENT}

In Chapter 7, we found that EE animals outperformed their standard housed counterparts after a $24 \mathrm{~h}$ ORT interval. These findings are in accordance with previous studies (Bechara \& Kelly, 2013; Bruel-Jungerman, Laroche, \& Rampon, 2005; Pamplona, Pandolfo, Savoldi, Prediger, \& Takahashi, 2009; Simpson \& Kelly, 2011, 
2012) demonstrating that the simplification of living conditions of laboratory animals for standardization purposes diminishes cognitive performance.

In the $24 \mathrm{~h}$ interval vardenafil was not effective in improving cognition in EE animals, however, we found that it was modestly effective in SOC animals, and fully improved memory in SOL animals. Furthermore, the most effective dose in SOC animals was lower and the effective dose-range was smaller compared to SOL animals.

After the $48 \mathrm{~h}$ retention interval, the animals from the different groups did not discriminate between the novel and familiar object, when treated with vehicle. Even after $48 \mathrm{~h}$, memory performance in SOL $(1 \mathrm{mg} / \mathrm{kg})$ and SOC $(0.3 \mathrm{mg} / \mathrm{kg})$ animals was still fully enhanced by vardenafil. However, like in the $24 \mathrm{~h}$ retention interval, none of the tested vardenafil doses caused memory improvement in EE rats in the $48 \mathrm{~h}$ retention interval. It could be argued that effects of vardenafil might have been at higher or even lower doses in the EE group. However, this is not very likely since lower doses may not reach significant brain levels to have a biological effect (see Chapter 4). High doses, on the other hand, are likely to reduce blood pressure (Reffelmann \& Kloner, 2006), which is generally not related to better memory performance (Duschek, Matthias, \& Schandry, 2005).

Our findings indicate that housing conditions have a marked effect on the effectiveness of vardenafil as a cognition enhancer. Not only did the effective dose seem to correspond negatively with the level of $\mathrm{EE}$, but also the effective dose range and magnitude of the cognition enhancement seemed to diminish as the level of $E E$ was increased.

Although we were unable to reveal the exact mechanism responsible for the effects of EE on vardenafil efficacy, an important finding was that PDE5 mRNA and protein expression were not altered between housing conditions. As PDE5 is the direct target of vardenafil, it is likely that alterations on a more general level caused the cognitive improvement in EE rats. Therefore, it is conceivable that the alterations in efficacy can be extrapolated to other classes of cognition enhancers (Franco \& Cedazo-Minguez, 2014). As a typical human is living in a social, diverse and stimulating environment it 
seems logical that EE living conditions in the laboratory will more closely resemble the living conditions of human subjects in clinical studies and therefore possess a greater predictive value. As drug development cost are ever increasing and current pharmacological CNS animal studies are notorious for their low predictive value (Kola \& Landis, 2004), it would be a sensible and economical approach to structurally screen new cognition enhancers in EE animals before advancing to clinical trials. An additional benefit is that EE would mean a substantial increase in the quality of life for laboratory animals. However, our findings with vardenafil will need to be replicated with other drugs that have been screened as cognition enhancers in animals to substantiate this notion. In this respect it would be especially interesting to investigate the effects of EE on drugs that have already proven their cognition enhancing potential in the clinic, for example donepezil (Wilkinson, 1999).

\section{GENERAL CONCLUSIONS}

The ORT is a reliable and sensitive technique to assess memory performance. The characteristics of the ORT offer various opportunities investigating the effects of drugs on different processes that underlie memory formation. The use of different retention intervals and application of injection time points showed that the acquisition process in rats does not stop immediately after $\mathrm{T} 1$ and that there is a short temporal overlap between acquisition and early consolidation processes. Moreover, using different test compound it was shown that different molecular mechanisms underlie these memory processes (including late consolidation). The application of these procedures may be useful for further characterization of new drugs that improve memory performance.

In the light of treating neurodegenerative disorders, episodic memory, is one of the most interesting memory types to investigate as it is the first type of memory affected in both normal and pathological aging (Tromp, Dufour, Lithfous, Pebayle, \& Despres, 2015). It is very likely that episodic memory is driving ORT performance in animals as we have seen in Chapter 2. Like Ennaceur suspected (2010) after the first exposure to an object, animals appear to make a temporal update during each following encounter with an object and it is this temporal aspect that fades within the first $8 \mathrm{~h}$ after T1 (Chapter 6). However, it will always remain a debate whether or not the ORT 
is a true measure of episodic memory as it does not address every single aspect of this memory type.

Our EE experiment showed that cognition enhancement vardenafil is very susceptible for environmental variability. This is a major problem for the translation of pre-clinical pharmacological findings to the clinic and raises questions about the predictive value of current highly standardized pre-clinical animal experiments. One the one hand, standardization is very useful in fundamental research as is helps to isolate and magnify very specific processes. On the other hand, it highly standardized are likely to give an exaggerated picture regarding the clinical effectives in a highly varied population. For this reason it might be very interesting to further investigate the general molecular mechanisms involved in the changes in drug efficacy with respect to $\mathrm{EE}$ and develop methods to screen promising clinical candidate drugs in an EE setting before the initiation of clinical trials.

In our studies we have identified the time specific involvement of CGMP and CAMP in the consolidation of memory, as well as their apparent involvement in acquisition related processes immediately after a learning trial. The characteristics of PDE4- and PDE5-I's makes them interesting as possible treatments for dementia as an increase in CAMP and CGMP is beneficial to both acquisition and consolidation of information. Another interesting aspect of PDE4- and PDE5-I's is that they appear to synergistically enhance cognition (Bollen et al., 2015) which means that combined treatment could lead to lower doses of each individual drug, limiting their individual adverse side effects.

Many cognition enhancing drugs fail in clinical development phases due to lack of efficacy and safety issues (Kola \& Landis, 2004). This indicates a major lack of fundamental knowledge about the underlying processes. It could take decades for science to provide the necessary fundamental understanding to develop truly effective cognition enhancing therapies. For the near future it would be interesting to reevaluate cognition enhancers that failed in clinical trials and investigate their combined effects on cognition. Compounds that have synergistic beneficial effects on cognition may be effective in drastically lower doses, increasing both efficacy and 
safety. Therefore a multi-drug approach may well be the way forward for the near future. 


\section{REFERENCES}

Abel, T., \& Lattal, K.M. (2001). Molecular mechanisms of memory acquisition, consolidation and retrieval. Current Opinion in Neurobiology, 11(2), 180-187.

Bailey, C.H., Kandel, E.R., \& Harris, K.M. (2015). Structural Components of Synaptic Plasticity and Memory Consolidation. Cold Spring Harb Perspect Biol, 7(7), a021758.

Bechara, R.G., \& Kelly, A.M. (2013). Exercise improves object recognition memory and induces BDNF expression and cell proliferation in cognitively enriched rats. Behav Brain Res, 245, 96-100.

Blake, M.G., Boccia, M.M., Krawczyk, M.C., \& Baratti, C.M. (2011). Scopolamine prevents retrograde memory interference between two different learning tasks. Physiol Behav, 102(3-4), 332-337.

Bollen, E., Akkerman, S., Puzzo, D., Gulisano, W., Palmeri, A., D'Hooge, R. (2015). Object memory enhancement by combining sub-efficacious doses of specific phosphodiesterase inhibitors. Neuropharmacology, 95, 361-366.

Bollen, E., Puzzo, D., Rutten, K., Privitera, L., De Vry, J., Vanmierlo, T. (2014). Improved Long-Term Memory via Enhancing CGMP-PKG Signaling Requires CAMP-PKA Signaling. Neuropsychopharmacology.

Bruel-Jungerman, E., Laroche, S., \& Rampon, C. (2005). New neurons in the dentate gyrus are involved in the expression of enhanced long-term memory following environmental enrichment. European Journal of Neuroscience, 21, 513-521.

Devan, B.D., Pistell, P.J., Daffin, J.L.W., Nelson, C.M., Duffy, K.B., Bowker, J.L. (2007). Sildenafil citrate attenuates a complex maze impairment induced by intracerebroventricular infusion of the NOS inhibitor N[omega]-nitro-Larginine methyl ester. European Journal of Pharmacology, 563(1-3), 134-140.

Devan, B.D., Sierra-Mercado, J.D., Jimenez, M., Bowker, J.L., Duffy, K.B., Spangler, E.L. (2004). Phosphodiesterase inhibition by sildenafil citrate attenuates the learning impairment induced by blockade of cholinergic muscarinic receptors in rats. Pharmacology Biochemistry and Behavior, 79(4), 691-699.

Dix, S.L., \& Aggleton, J.P. (1999). Extending the spontaneous preference test of recognition: evidence of object-location and object-context recognition. Behav Brain Res, 99(2), 191-200.

Domek-Lopacinska, K., \& Strosznajder, J.B. (2008). The effect of selective inhibition of cyclic GMP hydrolyzing phosphodiesterases 2 and 5 on learning and memory processes and nitric oxide synthase activity in brain during aging. Brain Research, 1216, 68-77. 
Duschek, S., Matthias, E., \& Schandry, R. (2005). Essential hypotension is accompanied by deficits in attention and working memory. Behav Med, 30(4), 149-158.

Ennaceur, A. (2010). One-trial object recognition in rats and mice: Methodological and theoretical issues. Behavioural Brain Research, 215(2), 244-254.

Ennaceur, A., \& Delacour, J. (1988). A new one-trial test for neurobiological studies of memory in rats. 1: Behavioral data. Behav Brain Res, 31(1), 47-59.

Franco, R., \& Cedazo-Minguez, A. (2014). Successful therapies for Alzheimer's disease: why so many in animal models and none in humans? Front Pharmacol, 5, 146.

Habib, R., Mclntosh, A.R., Wheeler, M.A., \& Tulving, E. (2003). Memory encoding and hippocampally-based novelty/familiarity discrimination networks. Neuropsychologia, 41(3), 271-279.

Hitchcock, S.A., \& Pennington, L.D. (2006). Structure-brain exposure relationships. J Med Chem, 49(26), 7559-7583.

Hughes, R.N. (2007). Neotic preferences in laboratory rodents: Issues, assessment and substrates. Neuroscience \& Biobehavioral Reviews, 31(3), 441-464.

Izquierdo, I., Bevilaqua, L.R., Rossato, J.I., Bonini, J.S., Medina, J.H., \& Cammarota, M. (2006). Different molecular cascades in different sites of the brain control memory consolidation. Trends Neurosci, 29(9), 496-505.

Klinkenberg, I., \& Blokland, A. (2010). The validity of scopolamine as a pharmacological model for cognitive impairment: a review of animal behavioral studies. Neurosci Biobehav Rev, 34(8), 1307-1350.

Kola, I., \& Landis, J. (2004). Can the pharmaceutical industry reduce attrition rates? Nat Rev Drug Discov, 3(8), 711-715.

Krause, W., \& kuhne, G. (1988). Pharmacokinetics of rolipram in the rhesus and cynomolgus monkeys, the rat and the rabbit. Studies on species differences. Xenobiotica, 18, 561-571.

Lepage, M., Habib, R., Cormier, H., Houle, S., \& Mclntosh, A.R. (2000). Neural correlates of semantic associative encoding in episodic memory. Cognitive Brain Research, 9(3), 271-280.

Lepage, M., McIntosh, A.R., \& Tulving, E. (2001). Transperceptual Encoding and Retrieval Processes in Memory: A PET Study of Visual and Haptic Objects. Neurolmage, 14(3), 572-584.

Matsui, K., Mishima, M., Nagai, Y., Yuzuriha, T., \& Yoshimura, T. (1999). Absorption, Distribution, Metabolism, and Excretion of Donepezil (Aricept) after a Single Oral Administration to Rat. Drug Metabolism and Disposition, 27(12), 14061414. 
Matsui, K., Mishima, M., Nagai, Y., Yuzuriha, T., \& Yoshimura, T. (1999). Absorption, distribution, metabolism, and excretion of donepezil (Aricept) after a single oral administration to Rat. Drug Metab Dispos, 27(12), 1406-1414.

Pamplona, F.A., Pandolfo, P., Savoldi, R., Prediger, R.D., \& Takahashi, R.N. (2009). Environmental enrichment improves cognitive deficits in Spontaneously Hypertensive Rats (SHR): relevance for Attention Deficit/Hyperactivity Disorder (ADHD). Progress in Neuropsychopharmacology \& Biological Psychiatry, 33, 1153-1160.

Prickaerts, J., de Vente, J., Honig, W., Steinbusch, H.W., \& Blokland, A. (2002). cGMP, but not CAMP, in rat hippocampus is involved in early stages of object memory consolidation. Eur J Pharmacol, 436(1-2), 83-87.

Prickaerts, J., Sik, A., van der Staay, F.J., de Vente, J., \& Blokland, A. (2005). Dissociable effects of acetylcholinesterase inhibitors and phosphodiesterase type 5 inhibitors on object recognition memory: acquisition versus consolidation. Psychopharmacology (Berl), 177(4), 381-390.

Prickaerts, J., van Staveren, W.C.G., Sik, A., Markerink-van Ittersum, M., Niewöhner, U., van der Staay, F.J. (2002). Effects of two selective phosphodiesterase type 5 inhibitors, sildenafil and vardenafil, on object recognition memory and hippocampal cyclic GMP levels in the rat. Neuroscience, 113(2), 351-361.

Rasch, B.H., Born, J., \& Gais, S. (2006). Combined Blockade of Cholinergic Receptors Shifts the Brain from Stimulus Encoding to memory Consolidation Journal of Cognitive Neuroscience, 18(5), 793-802.

Reffelmann, T., \& Kloner, R.A. (2006). Cardiovascular effects of phosphodiesterase 5 inhibitors. Curr Pharm Des, 12(27), 3485-3494.

Rush, D.K. (1988). Scopolamine amnesia of passive avoidance: A deficit of information acquisition. Behavioral and Neural Biology, 50(3), 255-274.

Rutten, K., Prickaerts, J., \& Blokland, A. (2006). Rolipram reverses scopolamineinduced and time-dependent memory deficits in object recognition by different mechanisms of action. Neurobiol Learn Mem, 85(2), 132-138.

Rutten, K., Prickaerts, J., Hendrix, M., van der Staay, F.J., Sik, A., \& Blokland, A. (2007). Time-dependent involvement of CAMP and cGMP in consolidation of object memory: studies using selective phosphodiesterase type 2, 4 and 5 inhibitors. Eur J Pharmacol, 558(1-3), 107-112.

Shaffer, C.L., \& John, E.M. (2010). Chapter 4 - Defining Neuropharmacokinetic Parameters in CNS Drug Discovery to Determine Cross-Species Pharmacologic Exposure "Response Relationships Annual Reports in Medicinal Chemistry (Vol. Volume 45, pp. 55-70): Academic Press.

Simpson, J., \& Kelly, J.P. (2011). The impact of environmental enrichment in laboratory rats- Behavioural and neurochemical aspects. Behavioural Brain Research, 222(1), 246-264. 
Simpson, J., \& Kelly, J.P. (2012). The effects of isolated and enriched housing conditions on baseline and drug-induced behavioural responses in the male rat. Behavioural Brain Research, 234(2), 175-183.

Spangler, E.L., Chachich, M.E., \& Ingram, D.K. (1988). Scopolamine in rats impairs acquisition but not retention in a 14-unit T-maze. Pharmacology Biochemistry and Behavior, 30(4), 949-955.

Tromp, D., Dufour, A., Lithfous, S., Pebayle, T., \& Despres, O. (2015). Episodic memory in normal aging and Alzheimer disease: Insights from imaging and behavioral studies. Ageing Res Rev.

van Donkelaar, E.L., Rutten, K., Blokland, A., Akkerman, S., Steinbusch, H.W.M., \& Prickaerts, J. (2008). Phosphodiesterase 2 and 5 inhibition attenuates the object memory deficit induced by acute tryptophan depletion. European Journal of Pharmacology, 600(1-3), 98-104.

van Goethem, N.P., Rutten, K., van der Staay, F.J., Jans, L.A.W., Akkerman, S., Steinbusch, H.W.M. (2012). Object recognition testing: Rodent species, strains, housing conditions, and estrous cycle. Behavioural Brain Research, 232(2), 323-334.

Wilkinson, D.G. (1999). The pharmacology of donepezil: a new treatment of Alzheimer's disease. Expert Opin Pharmacother, 1(1), 121-135.

Winters, B.D., Saksida, L.M., \& Bussey, T.J. (2006). Paradoxical facilitation of object recognition memory after infusion of scopolamine into perirhinal cortex: implications for cholinergic system function. J Neurosci, 26(37), 9520-9529.

Winters, B.D., Saksida, L.M., \& Bussey, T.J. (2008). Object recognition memory: Neurobiological mechanisms of encoding, consolidation and retrieval. Neuroscience \& Biobehavioral Reviews, 32(5), 1055-1070.

Young, S.L., Bohenek, D.L., \& Fanselow, M.S. (1995). Scopolamine impairs acquisition and facilitates consolidation of fear conditioning: differential effects for tone vs context conditioning. Neurobiol Learn Mem, 63(2), 174-180. 


\section{SUMMARY}


Phosphodiesterases (PDE's) are regulatory enzymes that hydrolyze the cyclic messenger molecules CGMP and CAMP which are involved in early and late consolidation processes, respectively. A large body of pre-clinical research has shown that phosphodiesterase inhibitors (PDE-I's) have the potential to enhance memory in animals. However, human studies into the effects of PDE-I's have not yet been very succesfull at replicating these findings. The aim of this thesis was to extend our knowledge about the pharmacology of PDE4-I rolipram and the PDE5-I vardenafil and further our understanding about the temporal characteristics of CGMP and CAMP signaling in memory formation. Combining behavioral data from the object recognition task (ORT) with the pharmacokinetic (PK) profiles of our test drugs we were able to accurately determine the active windows of CAMP and CGMP in earlyand late consolidation processes. Furthermore, we investigated the expression of memory improvement over time and the effects of environmetal conditions on drug efficacy in an attempt to find explanations for the discrepancies between pre-clinical PDE-I work and human studies.

\section{CHAPTER 1}

In this chapter we provide a general introduction into declarative memory and the the impact of cognitive empairment on our society. Furthermore we discribe some of the biological processess that are believed to be underlying memory, and the way in which PDE's are involved in these processes. Next, we adress how PDE-I's can be applied to manipulate these memory processes and enhance cognition and finally, we explain how the ORT can be used to separately investigate different stages of memory formation.

\section{CHAPTER 2 AND 3}

In this thesis we exclusively used the ORT for the assessment of memory performance. The ORT is a widely used method to assess memory performance in animals. However, there are many different variants of the ORT and there is debate about the exact memory type that is measured and the relationship between exploration and 
discrimination measures. To obtain a proper understanding about our ORT setup, we performed a meta-analysis of over 50 studies with Wistar rats that were performed in our lab. We found that the ORT is a sensitive task, which only requires a short learning trial for the reliable assessment of memory performance. In terms of analysis the actual occurrence of memory is assessed by comparing the discrimination measure to zero, preferably including realistic level of variation. Additionally, effects on memory performance should be assessed by comparison with a vehicle condition. We found that the relative discrimination measures $\mathrm{d} 2$ and $\mathrm{d} 3$ are very robust against exploration differences, in contrast to the absolute measure (d1) which has a strong positive correlation with the level of exploration. Although naïve animals are instinctively able to successfully discriminate between objects, it is preferable to familiarize the animals to the ORT prior to the actual experiment. Differences in object preference between condition including truly novel and relatively novel objects suggest that rats retain information about prior trials over long periods of time.

\section{CHAPTER 4}

There are not many publications about the PK properties of the PDE5-I vardenafil in rats, especially with regards to oral administration. Hence we performed a pharmacokinetic analysis using our preferred administration route for vardenafil (PO). We found that the drug was detectable in the brain within 2 min and unbound brain levels of vardenafil were above IC50 between 10-60 min after administration. The highest central concentration of vardenafil was measured 20 min after administration. Comparing behavioral data from intracerebroventricular (ICV) and oral vardenafil injections in a cholinergic deficit model and interpreting the PK data against a $1 \mathrm{~h}$ and a 10 min retention intervalr, our data suggest that PDE5 is involved in acquisition processes, which may linger for at least 4-6 min after learning. In addition, the effectiveness of very small amounts of ICV-administered vardenafil provides further evidence for the notion that PDE5-Is improve memory via a central mechanism. 


\section{CHAPTER 5}

In addition to the PDE5-I vardenafil, we investigated the pharmacokinetic profiles of the PDE4-I rolipram and AChE-I donepezil, in order to further elaborate the signaling windows of acetylcholine (Ach), CGMP and CAMP during memory consolidation in vivo. PK measurements revealed that PO andministered donepezil was detectable in the brain within $4 \mathrm{~min}$ after injection. Free brain concentrations above IC50 were reached between 10-60 min after administration and the highest free brain concentration was measured $20 \mathrm{~min}$ after the oral injection. In addition, IP administered rolipram was detected in the brain 2 min after injection and reached unbound brain concentrations above IC50 between 4-60 min after injection. The highest central concentration of rolipram was detected at 6 min after injection. Combining the data from the PK measurements to the behavioral data obtained in the ORT using a $1 \mathrm{~h}$ interval (with a scopolamine-induced short-term memory deficit) or a $24 \mathrm{~h}$ interval (natural forgetting of long-term memory) leads to the suggestion that acquisition processes continue for 6-12 min after the learning trial where they share a short common time frame of with early consolidation process. These acquisition processes also seemed to be affected by vardenafil and rolipram, possibly via an increase in CGMP/CAMP mediated ACh release. Finally, our data suggests that there is a critical window in memory consolidation for cGMP signaling within 47-55 min, and CAMP signaling between 3 to $5.5 \mathrm{~h}$ after the learning trial.

\section{CHAPTER 6}

It is known that long term memories (LTM) have to be physically incorporated in the synaptic architecture of the brain and that memories are not constant over time. In this chapter we investigated natural forgetting in the ORT in the first $24 \mathrm{~h}$ hours after learning and consequently, within these initial $24 \mathrm{~h}$, studied the expression profiles of object memory enhanced by PDE5-I vardenafil and PDE4-I rolipram. Rats were tested at different retention intervals ranging from $1 \mathrm{~h}$ to $24 \mathrm{~h}$, using a fixed time point for administration. Object discrimination performance of all groups time-dependently decreased up to an interval of $8 \mathrm{~h}$. We discovered that only after a $10 \mathrm{~h}$ retention 
interval the memory improving effects of vardenafil and rolipram started to emerge. This delayed manifestation of drug-enhanced memory suggests that two separate memory mechanisms are at play, a quick transient form of memory and a more stable memory form that requires several hours to develop. Furthermore, as both vardenafil and rolipram displayed identical memory expression profiles it appears that the CGMP and cAMP signaling pathways converge on the same downstream target.

\section{CHAPTER 7}

Many pre-clinical studies that have shown the potential of PDE-Is a cognition enhancing drugs. However, there convincing evidence about their effectiveness in humans has yet to be found. To explore whether environmental factors can partly explain the discrepancy between human- and animal studies we investigated the effects of environmental enrichment (EE) on the efficacy of PDE5-I vardenafil. Rats were either housed solitarily (SOL) or socially (SOC) under standard conditions, or socially in an EE. We found that EE animals were able remembered object information over longer periods of time in the vehicle conditions compared to SOL and SOC animals. However, vardenafil only improved object memory in SOL and SOC animals. Furthermore, the most effective dose in was lower and the effective dose-range was smaller in SOC animals compared to SOL animals. Our findings indicate that housing conditions have a marked effect on the effectiveness of vardenafil as a cognition enhancer. As PDE5 mRNA and protein expression were not altered between housing conditions it is conceivable that environmentally induced alterations in efficacy may also apply to other classes of cognition enhancers.

\section{CHAPTER 8}

In the final chapter of this thesis we discussed our main findings starting with the methodology of the ORT. We discuss how memory stages can be separately assessed using the ORT and the importance of the timing or pharmacological manipulation in relation to the learning- and test-trial. In the following section our findings about the pharmacology of donepezil, vardenafil and rolipram are discussed after which the PK 
data are combined with our behavioral findings to determine the active windows of ACh, cGMP and CAMP. Finally, we discuss the implications of the time-dependency of the expression of pharmacologically enhanced memory and environmental influences on drug efficacy. In the final part of our discussion shed light on possible future directions and the potential benefits of multi-drug treatment for cognitive impairments. 


\section{APPENDICES}

VALORISATION ADDENDUM

About the AUthor

LIST OF PUBLICATIONS

DANKWOORD 


\section{VALORISATION ADDENDUM}

Amnesia and cognitive impairment are becoming more common, especially in the aging western societies. Although cognitive decline is the hallmark symptom of Alzheimer's disease (AD) there is a host of other neuropsychiatric disorders that are associated with it. These include epilepsy, posttraumatic stress disorder (PTSD), depression, schizophrenia, Korsakoff's syndrome, Parkinson's disease and Huntington's disease. In addition, it may also be induced by malnutrition, sleep deprivation, severe stress, traumatic brain injuries (TBI) and stroke.

The tremendous burden that cognitive impairments pose on the quality of life of those who suffer from these afflictions is obvious. However, the psychological impact of cognitive impairment goes far beyond the individual, as patients become heavily dependent on relatives and professional caregivers. Furthermore, the economic burden on society is enormous. It is estimated that 5.2 million Americans currently have $A D$ and this number is expected to grow to 13.8 million around 2050. Worldwide, the number of people suffering from dementia in 2050 is estimated to be 115 million. In the US alone, the total costs in 2014 for healthcare for people aged 65 years and older with dementia are calculated to be $\$ 214$ billion. In 2013 , more than 15 million family members and other unpaid caregivers were estimated to have provided 17.7 billion hours of care to people with $A D$ and other dementias, an additional indirect cost valued around $\$ 220$ billion in man hours. These examples underline the magnitude of the social and economic costs that cognitive impairment has on our society and the urgent need to develop effective treatments.

The paradoxical issue about the current pharmacological treatments for Alzheimer's disease is that they are all focused on the manipulation of a single neurotransmitter system while it is known that there are numerous other molecules involved in memory formation. At present, the only approved drug treatments are either focused on enhancing cholinergic neurotransmission by inhibiting acetylcholinesterase, like donepezil, or glutamatergic receptor blocking. The effects of these treatments are largely symptomatic and have no significant impact on the long term-progression of 
the disease. In order to develop more effective treatments, there is a great need to explore alternative neurobiological targets, for instance the different PDE families.

PDEs are modulatory enzymes that regulate the intracellular levels of cyclic guanosine monophosphate (cGMP) and/or cyclic adenosine monophosphate (cAMP), which are important molecules in signal transduction related to memory formation. Several PDE families have been implicated in memory processes; amongst these are the PDE2, PDE4 and PDE5 families, for which specific pharmacological inhibitors (PDE-Is) have been developed. Two of these drugs are the PDE4 inhibitor rolipram and the PDE5 inhibitor vardenafil (Levitra). Although these PDE-Is were initially developed for diseases like chronic obstructive pulmonary disease (COPD) and erectile dysfunction, respectively, evidence from animal studies indicates that these drugs also have cognition enhancing properties. On the other hand, in human studies evidence for cognition enhancing properties of PDE-Is still has not been convincingly confirmed yet. These translational difficulties are not specific for PDE-Is, but are characteristic for the development of cognition enhancers in general. In order to achieve a better predictive validity we need to develop a more profound understanding of animal cognition, the way it is influenced by environmental factors and how to properly interpret the results from cognitive assessments. In addition, we need to invest more resources in basal scientific research into the biological processes underlying cognition so that we are better equipped to determine viable cognition enhancing treatments.

The former Nobel Prize winner Eric Kandel underlined the importance of behavioral research in his book 'In search of Memory: The Emergence of a New Science of Mind'. As he stated; "The results of that work had shown that different patterns of stimulation alter the strength of synaptic connections in different ways. But Tauc and I had not examined how an actual behavior is changed and therefore had no evidence that learning really relies on changes in synaptic strength." It is crucial to translate molecular findings into behavioral observations and also, to correctly interpret these behavioral changes in a meaningful way.

In this thesis, I structurally used the Object Recognition Task (ORT) to measure cognitive effects of drugs on Rats. Many different variants of the ORT are used and 
there is little consensus about the correct way to analyze the readout parameters or even what exact type of memory is being measured in the ORT. We extensively investigated a large data set to establish the most appropriate way to perform the experiments and interpret the results. This way we optimized the scientific revenue of our experiments. By publishing these results in specialized journals, we contributed to the general understanding of this behavioral task and the standardization of this task across laboratories around the world.

Another important factor to take into account when translating animal studies to humans is the difference in drug pharmacokinetics between animal species. This is important because the target molecules of cognition enhancing drugs have different active time windows during the formation memories. Therefore, it is of the utmost importance to accurately determine the pharmacokinetic (PK) properties of these drugs, especially when experimenting with a one-trial learning task (like the ORT). Furthermore, these PK properties are dependent on the administration route that is used. In the case of rolipram and vardenafil we are the first group which published the PK properties of orally administered vardenafil and rolipram administered intra peritoneally, in the rat, over the first hour post-administration. These PK data helped us determine the active windows of the PDE-regulated CGMP and CAMP signaling during memory formation, which gave us information about possible interactions between acquisition and (early) consolidation processes during memory formation. Our observations confirmed previously reported molecular findings. Thus, we are the first to reveal the complete active windows of CAMP and CGMP in vivo.

A better understanding about the pharmacokinetic properties of a drug helps us to also better understand the characteristics of its target(s). In the same way it is very relevant to properly understand the expression of its behavioral effects. In this thesis we show that the expression of memory is not stable over time, but disappears for a certain amount of time before re-appearing again. Hopefully, this knowledge will assist in the development better test protocols, so that promising effects of new treatment candidates will not be overlooked in a pre-clinical phase. The expression 
gap may also be present in human memory, and therefore it would be interesting to further investigate this possibility.

Finally, there is a growing concern about animal welfare. This is a valid concern as stress and discomfort are certain to affect the outcome of behavioral studies. A great deal of discomfort applied to laboratory animals is due to the large extent of impoverishment of their housing conditions due to standardization. We demonstrate that animals living in discomfort and unnatural circumstances have poorer cognitive capabilities compared to their counterparts living in more natural circumstances. We also demonstrate that these living conditions greatly affect the cognition enhancing effect of vardenafil. An effect that is likely caused by more basic underlying physiological processes and hence affects the translational value of pre-clinical studies towards cognition enhancing drugs in general. Considering enriched housing for cognition studies in animals will greatly increase animal welfare as well as enhance the predictive validity of the results found. Together with the refinements of the ORT methodology and analyses we hope to contribute to a qualitatively better and more humane way of performing animal research. 


\section{ABOUT THE AUTHOR}

Sven Akkerman was born in Utrecht on May 15th, 1983 in Utrecht, and grew up in Amerongen, the Netherlands. In 2001 he finished his pre-university education (VWO) at the Rembrandt College in Veenendaal. In the same year, he moved to Maastricht and started studying Psychology. In 2007 he obtained his Bachelors Degree in 'Biological Psychology' and continued to obtain his Masters Degree in 'Neuropsychology' at Maastricht University, in 2008. His research master internship, entitled 'The effects of selective PDE2 and PDE5 inhibitors Bayer 60-5770 and Vardenafil on object memory impaired by acute tryptophan depletion in the rat', was conducted at the School for Mental Health and Neuroscience at Maastricht University, where he started working as a scientific employee in the end of 2008. In the summer of 2011 he started working as a PhD-student at the School for Mental health and Neuroscience in the department Psychiatry and Neuropsychology of the faculty of Health Medicine and Life Sciences at Maastricht University, under the supervision of Dr. Jos Prickaerts, Dr. Arjan Blokland and Prof. Dr. Harry Steinbusch. These efforts accumulated into this thesis. Sven Akkerman is currently living in Utrecht (city). 


\section{LIST OF PUBLICATIONS}

Vanmierlo, T., Creemers, P., Akkerman, S., van Duinen, M., Sambeth, A., De Vry, J., Uz, T., Blokland, A., Prickaerts, J.(2016). The PDE4 inhibitor roflumilast improves memory in rodents at non-emetic doses. Behavioural Brain Research, 303, 26-33

Akkerman, S., Blokland, A., Klinkenberg, I., Steinbusch, H.W.M., Prickaerts, J. (2016). Possible overlapping time frames of acquisition and consolidation phases in object memory processes: a pharmacological approach. Learning \& Memory, 23,29-37

Akkerman, S., Blokland, A., van Goethem, N.P., Cremers, P., Shaffer, C.L., Osgood, S., Steinbusch, H.W.M., Prickaerts, J.(2015). PDE5 inhibition improves acquisition processes after learning via a central mechanism. Neuropharmacology, 97,233-239

Akkerman, S., Bollen, E., Puzzo, D., Palmeri, A., D'Hooge, R., Balschun, D., Steinbusch, H.W.M., Blokland, A., Prickaerts, J. (2015). Object memory enhancement by combining sub-efficacious doses of specific phosphodiesterase inhibitors. Neuropharmacology, 95, 361-366

Akkerman, S., Prickaerts, J., Bruder, A.K., Wolfs, K.H.M., de Vry, J., Vanmierlo, T., Steinbusch, H.W.M., Blokland, A. (2014). PDE5 inhibition improves object memory in standard housed rats but not in rats housed in an enriched environment: implications for memory models? PLOS ONE, Nov 5, 9(11)

Akkerman, S., Blokland, A., Prickaerts, J. (2014). Mind the gap: Delayed manifestation of long-term object memory improvement by phosphodiesterase inhibitors. Neurobiology of Learning and Memory, 109, 139-143

Bollen, E., Vanmierlo, T., Akkerman, S., Wouters, C., Steinbusch, H.W.M., Prickaerts, J. (2013). 7,8-Dihydroxyflavone improves memory consolidation processes in rats and mice. Behavioural Brain Research, 257, 8-12

van Donkelaar, E.L., Prickaerts, J., Akkerman, S., Rutten, K., Steinbusch, H.W.M., Blokland, A. (2013). No effect of acute tryptophan depletion on phosphodiesterase inhibition-related improvements of short-term object memory in male Wistar rats. Acta Psychiatrica Scandinavica, 128(2), 107-113

Akkerman, S., Blokland, A., Reneerkens, O., van Goethem, N.P., Bollen, E., Gijselaers, H.J., Lieben, C.K., Steinbusch, H.W., Prickaerts, J.(2012). Object recognition testing: methodological considerations on exploration and discrimination measures. Behavioral Brain Research, 232(2), 335-47

Akkerman, S., Prickaerts, J., Steinbusch, H.W., Blokland, A. (2012). Object recognition testing: statistical considerations. Behavioral Brain Research, 232(2), 317-22 
van Goethem, N.P., Rutten, K., van der Staay, F.J., Jans, L.A., Akkerman, S., Steinbusch, H.W., Blokland, A., van't Klooster, J., Prickaerts, J.(2012). Object recognition testing: rodent species, strains, housing conditions, and estrous cycle. Behavioral Brain Research, 232(2), 323-34

Reneerkens, O.A., Rutten, K., Akkerman, S., Blokland, A., Shaffer, C.L., Menniti, F.S., Steinbusch, H.W.M., Prickaerts, J. (2012). Phosphodiesterase type 5 (PDE5) inhibition improves object recognition memory: indications for central and peripheral mechanisms. Neurobiology of Learning and Memory, 97(4), 370-9

de Bruin, N.M., Prickaerts, J., Lange, J.H., ,Akkerman, S., Andriambeloson, E., de Haan, M., Wijnen, J., van Drimmelen, M., Hissink, E., Heijink, L., Kruse, C.G. (2012). SLV330, a cannabinoid CB1 receptor antagonist, ameliorates deficits in the T-maze, object recognition and Social Recognition Tasks in rodents. Neurobiology of Learning and Memory, 93(4), 522-31

de Bruin, N.M., Prickaerts, J., van Loevezijn, A., Venhorst, J., de Groote, L., Houba, P., Reneerkens, O., Akkerman, S., Kruse, C.G. (2011). Two novel 5-HT6 receptor antagonists ameliorate scopolamine-induced memory deficits in the object recognition and object location tasks in Wistar rats. Neurobiology of Learning and Memory, 96(2), 392-402

van Donkelaar, E.L., Rutten, K., Blokland, A., Akkerman, S., Steinbusch, H.W.M., Prickaerts, J.(2008). Phosphodiesterase 2 and 5 inhibition attenuates the object memory deficit induced by acute tryptophan depletion. European $\begin{array}{llll}\text { Journal of } & \text { 600(1-3), 98-104 }\end{array}$ 


\section{DANKWOORD}

En dan rest alleen nog het dankwoord. Het minst wetenschappelijke, maar meest aandachtig gelezen onderdeel van ieder proefschrift. En terecht, want vele anderen hebben mede bijgedragen aan het tot stand komen van dit manuscript. Graag wil ik hier de gelegenheid benutten om deze mensen persoonlijk te bedanken.

Ten eerste wil ik mijn promoter, Prof. Dr Harry Steinbusch bedanken voor de mogelijkheid om te promoveren aan de school for Mental Health and Neuroscience.

Daarnaast natuurlijk mijn co-promotores, te beginnen met Jos Prickaerts. Jos, jij bent degene geweest die de potentie in mij zag en me de kans gunde om deze PhD te behalen. Je energie en enthousiasme zijn altijd een inspiratiebron voor me geweest en ik heb de afgelopen jaren veel van je geleerd. Twee zaken zullen me altijd bij blijven. 1. "A mouse is not a small rat!" en 2. De veelzijdigheid van (roestige) tapijtscharen!

Dan natuurlijk mijn tweede co-promotor, Arjan Blokland. Arjan, jij bent bijzonder belangrijk geweest bij het tot stand komen van dit proefschrift. Als echte gedragswetenschapper voelde je precies aan wanneer er even wat positieve/negatieve reinforcement nodig was. Bedankt voor alle raad en daad, het spammen en sparren, en de tijd die je erin hebt gestoken. Je bent een grote motivatiebron geweest, zeker met de laatste loodjes.

Graag wil ik ook mijn officieuze mentrix nog even in het zonnetje zetten, Eva van Donkelaar. Toen wij elkaar voor het eerst ontmoetten leek het even water en vuur bij elkaar kwamen, ik citeer jouw eerste woorden als mijn stagebegeleidster: "Oh, jij bent toch niet zo'n Tragos koorbal hè?!". Dat was ik wel degenlijk, maar al snel bleek jij ook best van de traditionele stempel te zijn. Met ware ontgroeningsrituelen in het muizenhotel ("de timer loopt") en tryptofaan-depletie experimenten op krijsende ongehabitueerde ratten heb je me klaargestoomd voor de wetenschap. Nadat het ijs gebroken was hebben we gelukkig ook veel lol gehad. Eva, jij bent voor mij echt een voorbeeld geweest als wetenschapper en als vriendin, dankjewel. 
Alle collega's en vrienden van de Mood \& Cognition groep, bedankt voor de mooie jaren. We waren echt een hecht team en een schoolvoorbeeld van het feit dat je met samenwerken meer bereikt dan alleen. Annerieke, Eva, Jochen, Tim, Olga, Nick, Caroline (aka Juffrouw Jannie), Marlies, Kris, Marisela, Cindy, Pim, Jérome en natuurlijk ook Anke en Inge. Bedankt voor alle grappen, grollen en natuurlijk de inhoudelijke discussies. Jullie zijn een bron van plezier geweest op alle congressen, workshops, cursussen, pizzameetings, movienights, barbeques, lab day-outs en voetbal avonden. Het was een prachtige tijd en ik ga jullie zeker missen.

Hetzelfde geldt voor de rest van de School for Mental Health and Neuroschience. De beruchte club van 30; Jo, Alejandro en Gerard. Wat hebben we het mooi gevierd! Alle voetballers; Jochen, Ronald, Mark, Eshan, Pablo, Fabien, Leonidas, Youssef, Remco en Sabien, bedankt voor de mooie matches en de kamaraadschap! En ook alle andere mooie collegas waarmee ik het genoegen heb gehad om samen te mogen werken, Ali, Sonny, Evi, Michelle, Rinske, Bart, Gunter, Daniel, Bart, Lisa en Lisa, Dagmar, Nicole en iedereen die ik misschien per ongeluk vergeten ben.

Chris Shaffer and Sarah Osgood from Pfizer. Thank you for all the help and advice with the pharmacological determinations, your collaboration really was key to the success of this project.

Alle studenten die hebben meegeholpen aan mijn studies, en in het bijzonder AnnKristin, Kevin en Gada, bedankt voor jullie bijdrage.

Ook een woord van dank aan de groene brigade van het CPV. Richard, Inger, Harry, Rik, Alex, Paul en Saskia. Bedankt voor alle goede zorgen voor de proefdieren, de fijne samenwerking en de gezelligheid. Het is niet te onderschatten hoe belangrijk jullie zijn geweest voor de tot standkoming van dit proefschrift.

Daarnaast wil ik onze analisten, Hellen, Denise en Marjan bedanken voor hun bijdrage aan mijn onderzoek. Zonder een goede basis ben je nergens en ik overdrijf niet als ik zeg dat jullie de fundering zijn onder alle experimenten die ik over de jaren heb uitgevoerd. Nog een speciaal word van dank aan Marjanne Markerink, voor alle hulp 
bij het verschepen van de breinsamples naar China. Zonder jou waren ze de grens niet over gekomen.

Special thanks to Wiel Honig. Je bent echt een man van de oude stempel en van alle markten thuis. Of het nu in het lab is ("Korter of langer?"), een probleem met de laptop, of het hacken van een microscoop. Ik kon altijd bij je aankloppen.

Jaap Tolk en Floris Jansen, mijn paranimfen. Jullie zijn er al bij vanaf het eerste biertje op de Kastanjelaan, voordat ik ook maar een minuut in de collegebanken had doorgebracht. "Vrienden voor het leven maak je hier!", zeiden ze bij het H.D. Nexus en ze hadden gelijk. Vele jaren en avonturen later zijn we nog net zo hecht als tijdens onze studententijd en ik ben blij en trots dat ik jullie op deze speciale dag achter me heb staan.

Lieve familie, Pa, Ma, Jens, Bente, Opa en Oma. Het is mede door alle liefde, steun, hulp en interesse die ik over de de jaren heen van jullie heb gehad dat ik het zover heb kunnen schoppen. Jullie zijn altijd een bron van kracht, wijsheid en inspiratie voor me geweest en ik hou van jullie allemaal. Familie kun je niet kiezen, maar ik kan me geen betere wensen.

En dan tot slot, mijn lieve Rianne. Het is onmogelijk om in een paar woorden te beschrijven hoeveel je voor me betekent. Je bent een enorme steun voor me geweest en ik heb altijd op je kunnen bouwen. Je hebt mij gemotiveerd en geïnspireerd op momenten dat ik er doorheen zat en je bent altijd in me blijven geloven. Maar het zijn vooral je humor, liefde en vertrouwen waar ik enorm veel kracht uit haal. Jij bent de allerliefste van de allermaalste en ik kijk er enorm uit om met jou samen onze volgende uitdaging aan te gaan!

Hora Est! 